\title{
ASYMPTOTIC EXPANSIONS OF SOLUTIONS OF THE SIXTH PAINLEVÉ EQUATION
}

\author{
A. D. BRUNO AND I. V. GORYUCHKINA
}

\begin{abstract}
We obtain all asymptotic expansions of solutions of the sixth Painlevé equation near all three singular points $x=0, x=1$, and $x=\infty$ for all values of four complex parameters of this equation. The expansions are obtained for solutions of five types: power, power-logarithmic, complicated, semiexotic, and exotic. They form 117 families. These expansions may contain complex powers of the independent variable $x$. First we use methods of two-dimensional power algebraic geometry to obtain those asymptotic expansions of all five types near the singular point $x=0$ for which the order of the leading term is less than 1 . These expansions are called basic expansions. They form 21 families. All other asymptotic equations near three singular points are obtained from basic ones using symmetries of the equation. The majority of these expansions are new. Also, we present examples and compare our results with previously known ones.
\end{abstract}

\section{INTRODUCTION}

In 1884-1885 L. Fuchs 47] and H. Poincaré [70, 71, 72, suggested looking for differential equations whose solutions do not have movable critical points and cannot be expressed in terms of previously known functions. In $1889 \mathrm{~S}$. Kovalevskaya [36] had shown that the absence of movable critical points of solutions allows one to construct solutions analytically.

A singular point $x=x_{0}$ of a function $y(x)$ of a complex variable $x$ is called a critical singular point if the value of the function $y(x)$ changes as $x$ moves along the path surrounding $x_{0}$. A movable singular point of a solution of a differential equation is a singular point such that its position depends on initial conditions of the problem. For example for the solution $y=1 / \sqrt{x-x_{0}}$, where $x_{0}$ is an arbitrary constant, the point $x=x_{0}$ is a movable critical point. By a meromorphic function we mean a function whose only singularities in the finite part of the complex place are poles.

In 1887 E. Picard [68] suggested studying the following class of second order equations:

$$
y^{\prime \prime}=F\left(x, y, y^{\prime}\right)
$$

where the function $F$ is rational in $y$ and $y^{\prime}$ and meromorphic in $x$, and to find, among equations (11), those that have only immovable critical singular points. At the beginning of the 20th century P. Painlevé [65, 66, 67, and his students B. Gambier [49] and R. Garnier [50, 51] solved the problem formulated by Fuchs and Picard. They have found 50 canonical equations of the form (1) whose solutions have no movable critical singular

2010 Mathematics Subject Classification. Primary 34E05; Secondary 34M55.

The work was supported by the Russian Foundation of Fundamental Research (Project 08-01-00082) and the Foundation for the Assistance to Russian Science.

Editorial Note: The following text incorporates changes and corrections submitted by the authors for the English translation. 
points. Solutions of 44 of these equations could be expressed in terms of known (elementary or special) functions, and solutions of the remaining six equations determine new special functions, which are now called Painlevé transcendents.

The sixth Painlevé equation first appeared in the paper by R. Fuchs [48]. In has the form

$$
\begin{aligned}
y^{\prime \prime}= & \frac{\left(y^{\prime}\right)^{2}}{2}\left(\frac{1}{y}+\frac{1}{y-1}+\frac{1}{y-x}\right)-y^{\prime}\left(\frac{1}{x}+\frac{1}{x-1}+\frac{1}{y-x}\right) \\
& +\frac{y(y-1)(y-x)}{x^{2}(x-1)^{2}}\left[a+b \frac{x}{y^{2}}+c \frac{x-1}{(y-1)^{2}}+d \frac{x(x-1)}{(y-x)^{2}}\right]
\end{aligned}
$$

where $a, b, c, d$ are complex parameters, $x$ and $y$ are complex variables, $y^{\prime}=\frac{d y}{d x}$. It has three singular points $x=0, x=1$ and $x=\infty$, and is usually denoted P6. E. Picard 69] has found solutions of this equation in an explicit form for special values of four parameters: $a=b=c=0, d=\frac{1}{2}$. R. Garnier [51] was studying solutions of this equation without any restrictions on parameters.

A new wave of interest to Painlevé equations occurred in the 1970s after M. Ablowitz, A. Ramani, and H. Segur 1, 42, 43 discovered that integrable nonlinear partial differential equations are related to Painlevé equations (see also [35, 37]). For example, the sixth Painlevé equation is a reduction of the Ernst equation in general relativity. Nowadays, the followng problems for the Painleve equations are being studied: asymptotic behavior of solutions near singular points, local and global properties of solutions, rational and algebraic solutions, discretization, applications of Painlevé equations (mainly in physics).

In the present paper we study asymptotic expansions of solutions of the sixth Painlevé equation at the singular points $x=0,1, \infty$. Expansions in nonsingular points were described in [54, $\S 46]$, and, using power geometry, in [13, 24]. Similar studies were performed by many authors. S. Shimomura [73-76], M. Jimbo 61], H. Kimura 62, K. Okamoto 64 proved, using a variety of methods, existence and convergence of twoparameter families of expansions for solutions of the sixth Painlevé equation. In the book [54, $\S 46]$ by I. V. Gromak, I. Laine, and S. Shimomura the authors describe asymptotic expansions of solutions in integer powers of the independent variable. For some special values of parameters of the sixth Painlevé equation, B. Dubrovin and M. Mazzocco [46, 63, and also D. Guzzetti [55] obtained several initial terms of nonpower and exotic expansions. A comparison of these results with ours is presented at the end of the Introduction and in Section 2 of Chapter 4.

The study of asymptotic extensions and asymptotic properties of solutions of Painlevé equations near singular points consists of the following three main steps.

Step 1. To find formal solutions in the form of asymptotic expansions

$$
y=\sum_{k=0}^{\infty} \varphi_{k}(x),
$$

where $\varphi_{k+1}(x)=o\left(\varphi_{k}(x)\right)$, and formal asymptotics

$$
y \sim \sum_{k=0}^{m} \varphi_{k}(x),
$$

where the remainder is $o\left(\varphi_{m}(x)\right)$.

Step 2. To prove the existence of a solution with asymptotic expansion (3) or asymptotic behavior (4). Such a solution may be nonunique. 
Step 3. To find a relation between asymptotic behavior of the same solution near different singular points of the equation, and more specifically, between arbitrary constants of families of asymptotic expansions.

The main result of the present paper can be expressed as follows. For all values of the four complex parameters $a, b, c, d$ we find all those formal solutions (3) of equation (2) near three singular points $x=0,1, \infty$ that can be otained using two-dimensional power geometry.

According to 21]-23 three-dimensional power geometry also allows us to find asymptotic behavior of the first four Painlevé equations (in particular, elliptic asymptotics) that cannot be found using the two-dimensional power geometry. This may also be the case for P6. However, this is a subject of another paper.

The problem of existence of a solution with a given formal expansion is discussed in Section 7 of Chapter 1 from a general point of view. This is also a subject of a separate paper. Currently, not for every formal solution (3) the corresponding actual solution is found. However, there are no examples of a formal solution (3) for which it is known that the corresponding actual solution does not exist.

For the majority of formal or asymptotic solutions of Painlevé equations found earlier by ad hoc methods, the existence of actual solutions is known (also with the help of ad hoc methods).

The present paper does not address the problem of the relation of asymptotic properties of a solution of the equation P6 at different singular points (i.e., Step 3). This is also a topic for another paper.

Everywhere below by an asymptotic expansion of a solution of equation (2) we mean the expansion of its formal solution.

For $x \rightarrow 0$ let us consider asymptotic expansions of solutions of equation (2) of the form

$$
y=c_{r} x^{r}+\sum_{s} c_{s} x^{s}
$$

where the exponents $r$ and $s$ are complex numbers, $\operatorname{Re} s>\operatorname{Re} r$, and $\operatorname{Re} s$ increases.

We distinguish three types of expansion (5) (we assume that the number of exponents $s$ with the same real part $\operatorname{Re} s$ is finite):

Type 1. $c_{r}$ and $c_{s}$ are constant (power expansions).

Type 2. $c_{r}$ is constant, $c_{s}$ are polynomials in $\ln x$ (power-logarithmic expansions).

Type 3. $c_{r}$ and $c_{s}$ are power series in decreasing powers of $\ln x$ (complicated expansions).

Furthermore, we will distinguish three types of expansions of the form

$$
y=\sum_{\rho} c_{\rho} x^{\rho}+\sum_{s} c_{s} x^{s}
$$

where all $\operatorname{Re} \rho$ are equal, $\operatorname{Re} \rho<\operatorname{Re} s$, the first sum contains more than one summand, and the coefficients $c_{\rho}$ and $c_{s}$ are polynomials in $\ln x$ :

Type 4. The first sum in (6) contains a finite number of summands. For the largest and the smallest value of $\operatorname{Im} \rho$ the coefficients $c_{\rho}$ are constant. In such expansions the number of exponents $s$ with the same real part $\operatorname{Re} s$ is finite (semiexotic expansions). 
Let $\min (\operatorname{Im} \rho)$ in (6) be reached at $\rho=\rho_{*}$, and $\max (\operatorname{Im} \rho)$ at $\rho=\rho^{*}$. A semiexotic expansion (6) has two inverse expansions

$$
\left(y^{-1}\right)_{*}=c_{\rho_{*}}^{-1} x^{-\rho_{*}}\left(\sum_{\sigma} b_{\sigma} x^{\sigma}+\sum_{t} b_{t} x^{t}\right)
$$

and

$$
\left(y^{-1}\right)^{*}=c_{\rho^{*}}^{-1} x^{-\rho^{*}}\left(\sum_{\sigma} \tilde{b}_{\sigma} x^{\sigma}+\sum_{t} \tilde{b}_{t} x^{t}\right),
$$

where $\operatorname{Re} \sigma=0$.

Type 5. The first sum in (6) contains an infinite number of summands, but $\operatorname{Im} \rho$ is bounded either from above or from below. For the largest and the smallest value of $\operatorname{Im} \rho$ we have $c_{\rho}=$ const $\in \mathbb{C}$ (exotic expansions).

The inverse to an exotic expansion (6) is one of the expansions (7) or (8).

Type 6. The first sum in (6) contains an infinite number of summands and the values $\operatorname{Im} \rho$ are unbounded at both ends (superexotic expansions).

We note that expansions of Type 6 do not occur for the Painlevé equation P6.

Moreover, we assume that the argument of the complex variable $x$ is bounded from both sides.

One can similarly define types of asymptotic expansions as $x \rightarrow 1$ and $x \rightarrow \infty$.

Equation (2) has three symmetries that map singular points into one another. Therefore, we first solve the problem near $x=0$, and then, using symmetries, obtain asymptotic expansions near $x=\infty$ and $x=1$.

The paper consists of five chapters. In Chapter 1 we present methods and results of two-dimensional power geometry 2-26, 44 that are used in the paper. The leading term of the asymptotic expansion (5) is computed using the reduced equation that is formed by those terms of the initial equation that are leading terms for this expansion (make the largest contribution near the point under consideration). These equations are studied using graphs. The exponents $s$ of other terms of the expansion $c_{s} x^{s}$ are found algorithmically. Coefficients are computed consecutively. In $\S 1$ we describe methods to distinguish reduced equations and to compute power asymptotic expansions for solutions of the initial equation. In $\S 2$ we present methods to compute exponents of all terms of an asymptotic expansion with a power function as a leading term, and to determine the coefficients of such an expansion. In $\S[3$ we consider the existence of nonpower asymptotic expansions for solutions of the original equation and describe an algorithm to find such expansions. In $\S 4$ we present a method to compute complicated expansions (expansions with nonpower asymptotics). In $\S 5$ we determine exotic and semiexotic expansions and describe methods to compute these expansions. In $\S 6$ we present exponential asymptotic expansions and additional terms in expansions of Types 1, 2, 4 that do not occur in the case of equation P6. In $\S 7$ we present results about the existence of an actual solution with a given formal solution as the asymptotic expansion. We also formulate a general theorem about the convergence of a power expansion and prove it in a special case.

In Chapter 2 we look for asymptotic expansions of all five types for the sixth Painlevé equation as $a \cdot b \neq 0, x \rightarrow 0$, and $x \rightarrow \infty$. In $\S 1$ equation (2) is written as a differential sum. To do this we multiply it by $2 x^{2}(x-1)^{2} y(y-1)(y-x)$ and move the right-hand 


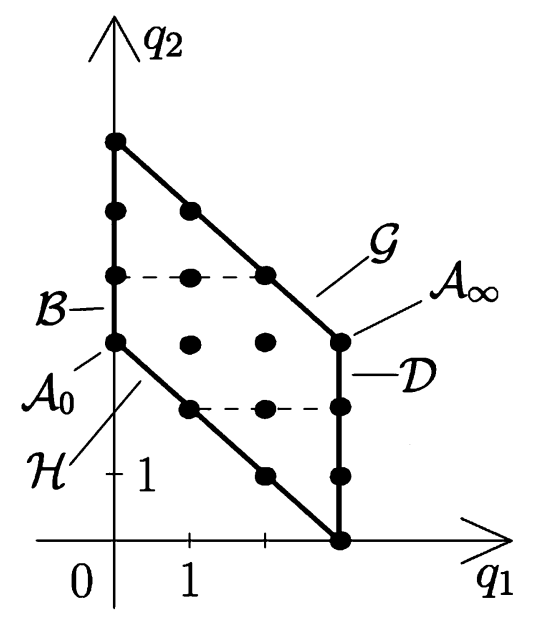

FiguRE 1. The polygon of equation (9) and notation for families of expansions corresponding to its vertices and edges for $a \cdot b \neq 0$.

side to the left. We obtain the equation

(9)

$$
\begin{aligned}
f(x, y) \stackrel{\text { def }}{=} & 2 y^{\prime \prime} x^{2}(x-1)^{2} y(y-1)(y-x) \\
- & \left(y^{\prime}\right)^{2}\left[x^{2}(x-1)^{2}(y-1)(y-x)+x^{2}(x-1)^{2} y(y-x)+x^{2}(x-1)^{2} y(y-1)\right] \\
+ & 2 y^{\prime}\left[x(x-1)^{2} y(y-1)(y-x)+x^{2}(x-1) y(y-1)(y-x)+x^{2}(x-1)^{2} y(y-1)\right] \\
- & {\left[2 a y^{2}(y-1)^{2}(y-x)^{2}+2 b x(y-1)^{2}(y-x)^{2}\right.} \\
& \left.+2 c(x-1) y^{2}(y-x)^{2}+2 d x(x-1) y^{2}(y-1)^{2}\right]=0 .
\end{aligned}
$$

Then we study the main properties of equation (9): the polygon of the equation, symmetries, and exceptional solutions $\mathcal{I}_{1}: y=0$ as $b=0, \mathcal{I}_{2}: y=1$ as $c=0, \mathcal{I}_{3}: y=x$ as $d=\frac{1}{2}, \mathcal{I}_{4}: y=\infty$ as $a=0$. The polygon of equation (9) and the notation for families of expansions corresponding to vertices and edges of this polygon are shown in Figure 1 .

In $\S 2$ we study those asymptotic expansions (5) of solutions near zero that correspond to the vertices of the polygon. It is shown that such solutions exist only for the vertex with coordinates $\left(q_{1}, q_{2}\right)=(0,3)$. The main result in Chapter 2 is as follows.

Theorem 2.2.1. For $x \rightarrow 0$ there exists a two-parameter (in $c_{r}$ and $r$ ) family of solutions with constant coefficients given by the formula

$$
\mathcal{A}_{0}: y=c_{r} x^{r}+\sum_{s} c_{s} x^{s}
$$

where the complex exponents are as follows: $r$ is arbitrary with $\operatorname{Re} r \in(0,1), s \in$ $\{r+l r+m(1-r) ; l, m \geq 0 ; l+m>0 ; l, m \in \mathbb{Z}\}$, and the complex coefficients are as follows: $c_{r}$ is an arbitrary complex constant, and the other $c_{s}$ are uniquely defined constants.

Expansions (2.2.6) converge for small $|x|$. The family $\mathcal{A}_{0}$ and its convergence were known earlier; see [60]-62], 64, and [73]- 78 . The family $\mathcal{A}_{0}$ exists for all values of the parameters and exhausts all expansions corresponding to the vertex $(0,3)$.

Throughout the article, ln means log. 
In $\S 3$ we analyze asymptotic expansions near zero that correspond to the left vertical edge of the polygon (see Figure 1). For the reader's convenience, the main result can be formulated as follows (in more detail than in Chapter 2).

Theorem 2.3.1. (a) For $x \rightarrow 0$ and $a \neq 0$ there exist two two-parameter (in $C_{11}$ and $\rho)$ families of exotic expansions of solutions with constant coefficients; these families are given by the formula

(2.3.4

$$
\begin{aligned}
\mathcal{B}_{0}^{\tau}: \quad y & =\frac{\rho^{2}}{\beta \cos ^{2}\left[\ln \left(C_{11} x\right)^{\gamma}\right]+\alpha \sin ^{2}\left[\ln \left(C_{11} x\right)^{\gamma}\right]}+\sum_{\operatorname{Re} s \geq 1} c_{s} x^{s} \\
& =x^{\rho}\left(c_{\rho}+\sum_{k=1}^{\infty} \tilde{c}_{k} x^{k \rho}\right)+\sum_{\operatorname{Re} s \geq 1} c_{s} x^{s}
\end{aligned}
$$

where $\rho \neq 0$ is an arbitrary purely imaginary constant, $s \in\{\rho+l \rho+m(1-\rho) ; l, m \geq 0$; $l+m>0 ; l, m \in \mathbb{Z}\}, \tau=\operatorname{sgn}(\operatorname{Im} \rho), \alpha+\beta=\frac{\rho^{2}-2 c+2 a}{2 a}, \alpha \beta=\frac{\rho^{2}}{2 a}, 2 \gamma=i \rho$, and $c_{\rho}$ and $C_{11}$ are arbitrary nonzero complex constants related to one another, $\tilde{c}_{k}$ and $c_{s}$ are uniquely determined constants. The families of inverse expansions $y^{-1}(x)$ for the families $\mathcal{B}_{0}^{+}$and $\mathcal{B}_{0}^{-}$coincide.

(b) For $0 \neq a \neq c \neq 0$ and $x \rightarrow 0$ there exist one-parameter families of expansions $\mathcal{B}_{i}$ and $\mathcal{B}_{i}^{\tau}, i=1,2, \tau= \pm 1$ that are given by one of the formulas (2.3.14), (2.3.18), (2.3.19) below, depending on the values of complex numbers $\theta_{1}=\sqrt{2 c}-\sqrt{2 a}$ and $\theta_{2}=\sqrt{2 c}+\sqrt{2 a}$.

Namely, if $\operatorname{Re} \theta_{i}=0$, then the families $\mathcal{B}_{i}^{\tau}$ are given by the formula (see (2.3.14), $(2.3 .40))$

$$
\mathcal{B}_{i}^{\tau}: \quad y=c_{0 i}+\sum_{s} c_{s i} x^{s}=\frac{c_{0 i}}{1-C_{7} x^{\tau \theta_{i}}}+\sum_{\operatorname{Re} s \geq 1} c_{s i} x^{s},
$$

where $s \in\left\{l+m \tau \theta_{i} ; l, m \in \mathbb{Z} ; l, m \geq 0 ; l+m>0\right\}$, the complex coefficients $c_{0 i}$ are given by

(2.3.6) $c_{0 i}=1+(-1)^{i} \sqrt{\frac{c}{a}}$,

$C_{7}$ is an arbitrary constant, and other constants $c_{s i}$ are uniquely determined.

Now let $\operatorname{Re} \theta_{i} \neq 0$ and $k_{i}=\theta_{i} \cdot \operatorname{sgn}\left(\operatorname{Re} \theta_{i}\right)$.

If $\operatorname{Re} \theta_{i} \neq 0$ and $\theta_{i} \notin \mathbb{Z}$, then the family $\mathcal{B}_{i}$ is given by the formula

$$
\mathcal{B}_{i}: \quad y=c_{0 i}+\sum_{s} c_{s i} x^{s},
$$

where $s$ runs over the set $\left\{l+m k_{i} ; l, m \in \mathbb{Z}, l, m \geq 0, l+m>0\right\}$, and the complex coefficients are as follows: $c_{0 i}$ is given by formula (2.3.6), $c_{k_{i} i}$ is arbitrary, and the remaining $c_{s i}$ are uniquely determined constants.

If $\theta_{i} \in \mathbb{Z} \backslash\{0\}$, then the family $\mathcal{B}_{i}$ is given by the formula

$$
\mathcal{B}_{i}: \quad y=c_{0 i}+\sum_{s=1}^{\infty} c_{s i}(\ln x) x^{s}
$$

where the coefficients are as follows: $c_{0 i}$ is given by formula (2.3.6), $c_{s i}$ with $s<k_{i}$ are constants, $c_{k_{i} i}=\alpha_{k_{i} i}+\beta_{k_{i} i} \ln x, \alpha_{k_{i} i}$ is an arbitrary constant, the coefficient $\beta_{k_{i} i}$ is a uniquely determined constant, and the remaining $c_{s i}$ with $s>k_{i}$ are uniquely determined polynomials in $\ln x$.

In the case $C_{7}=0$ expansions (2.3.14) are integral, i.e., $s \in \mathbb{Z}$; the family of these expansions is denoted $\mathcal{B}_{i}$. It coincides with a subfamily of the family (2.3.18) for $c_{k_{i} i}=0$, i.e., for $m=0$.

The family $\mathcal{B}_{2}$ exists for $a=c \neq 0$ as well. 
(c) For $a \cdot c \neq 0$ and $x \rightarrow 0$ there exist one-parameter families of complicated expansions, namely,

- $\quad$ the family $\mathcal{B}_{3}$, which exists for $a \neq c$ and is given by the formulas

$$
\mathcal{B}_{3}: \quad y=\psi_{0}+\sum_{\sigma=1}^{\infty} \psi_{\sigma} x^{\sigma},
$$

where

$(2.3 .28)$

$$
\psi_{0}=\frac{2}{c-a} \frac{1}{\ln ^{2} x}+\frac{c_{-3}}{\ln ^{3} x}+\sum_{s=4}^{\infty} \frac{c_{-s}}{\ln ^{s} x}=\frac{2(c-a)}{(c-a)^{2}\left(\ln x+C_{3}\right)^{2}-2 a},
$$

and the complex coefficients are as follows: $c_{-3}$ and $C_{3}$ are arbitrary complex constants and the remaining $c_{-s}$ are uniquely determined constants; $\psi_{\sigma}$ are series in decreasing powers of logarithms;

- the families $\mathcal{B}_{4}$ and $\mathcal{B}_{5}$, which exist for $a=c \neq 0$ and are given by the formulas

$$
\mathcal{B}_{3+j}: \quad y=\psi_{0 j}+\sum_{\sigma=1}^{\infty} \psi_{\sigma j} x^{\sigma}, \quad j=1,2,
$$

where

(2.3.57)

$$
\psi_{0 j}=(-1)^{j} \frac{1}{\sqrt{2 a}} \frac{1}{\ln x}+\frac{c_{-2 j}}{\ln ^{2} x}+\sum_{s=3}^{\infty} \frac{c_{-s j}}{\ln ^{s} x}=\frac{(-1)^{j}}{\sqrt{2 a} \ln x+C_{3}}, \quad j=1,2,
$$

with the following complex coefficients: $c_{-2 j}$ (i.e., $C_{3}$ ) is an arbitrary constant and the remaining $c_{-s j}$ are uniquely determined constants; $\psi_{\sigma j}$ are series in decreasing powers of logarithms.

(d) For $a \neq 0, c=0$ and $x \rightarrow 0$ there exist one-parameter families of expansions $\mathcal{B}_{6}$, $\mathcal{B}_{6}^{\tau}, \tau= \pm 1$, given by the formula

$$
y=1+c_{\rho} x^{\rho}+\sum_{s} c_{s} x^{s}
$$

where $\rho=\sqrt{2 a}$ with $\operatorname{Re} \sqrt{2 a}>0$ (the family of power expansions $\mathcal{B}_{6}$ ) or with $\operatorname{Re} \sqrt{2 a}=0$ (families of exotic expansions $\mathcal{B}_{6}^{\tau}$ ), where $s$ runs over the set $\{\rho+l \rho+m ; l, m \geq 0 ; l+$ $m>0 ; l, m \in \mathbb{Z}\}, c_{\rho}$ is an arbitrary nonzero constant, and all $c_{s}$ are arbitrary nonzero constants.

The families $B_{6}^{\tau}$ with $\tau=\operatorname{sgn}(\operatorname{Im} \rho)$ can also be written in the form

$$
y=\frac{1}{1-c_{\rho} x^{\rho}}+\sum_{\operatorname{Re} s \geq 1} c_{s} x^{s} .
$$

The families (2.3.18) and (2.3.71) converge for sufficiently small $|x|$. The families $\mathcal{B}_{1}-$ $\mathcal{B}_{6}, \mathcal{B}_{0}^{\tau}, \mathcal{B}_{1}^{\tau}, \mathcal{B}_{2}^{\tau}, \mathcal{B}_{6}^{\tau}$ with $\tau= \pm 1$ exhaust all expansions corresponding to the left vertical edge of the polygon in Figure 1. For families $\mathcal{B}_{1}, \mathcal{B}_{2}$, only subfamilies with constant coefficients and integer exponents were known before [54]. The families $\mathcal{B}_{0}^{\tau}, \mathcal{B}_{1}^{\tau}, \mathcal{B}_{2}^{\tau}, \mathcal{B}_{6}^{\tau}$, $\mathcal{B}_{3}-\mathcal{B}_{6}$ are new. The families $\mathcal{A}_{0}, \mathcal{B}_{1}-\mathcal{B}_{6}, \mathcal{B}_{0}^{\tau}, \mathcal{B}_{1}^{\tau}, \mathcal{B}_{2}^{\tau}, \mathcal{B}_{6}^{\tau}$ are basic families. Using the main symmetries of equation (2), one can obtain from them other families of expansions. In $\S 4$ we use symmetries to obtain from the basic families $\mathcal{B}_{1}-\mathcal{B}_{6}, \mathcal{B}_{0}^{\tau}, \mathcal{B}_{1}^{\tau}, \mathcal{B}_{2}^{\tau}, \mathcal{B}_{6}^{\tau}$ the families $\mathcal{H}_{1}-\mathcal{H}_{6}, \mathcal{H}_{0}^{\tau}, \mathcal{H}_{1}^{\tau}, \mathcal{H}_{2}^{\tau}, \mathcal{H}_{6}^{\tau}$ (as $x \rightarrow 0$ ) corresponding to the lower inclined edge of the polygon in Figure 11). In $\S 5$ another symmetry is applied to asymptotic expansions (as $x \rightarrow 0$ ) forming the families $\mathcal{A}_{0}, \mathcal{B}_{1}-\mathcal{B}_{6}, \mathcal{B}_{0}^{\tau}, \mathcal{B}_{1}^{\tau}, \mathcal{B}_{2}^{\tau}, \mathcal{B}_{6}^{\tau}, \mathcal{H}_{1}-\mathcal{H}_{6}, \mathcal{H}_{0}^{\tau}, \mathcal{H}_{1}^{\tau}, \mathcal{H}_{2}^{\tau}, \mathcal{H}_{6}^{\tau}$, which results in families $\mathcal{A}_{\infty}, \mathcal{G}_{1}-\mathcal{G}_{6}, \mathcal{G}_{0}^{\tau}, \mathcal{G}_{1}^{\tau}, \mathcal{G}_{2}^{\tau}, \mathcal{G}_{6}^{\tau}, \mathcal{D}_{1}-\mathcal{D}_{6}, \mathcal{D}_{0}^{\tau}, \mathcal{D}_{1}^{\tau}, \mathcal{D}_{2}^{\tau}, \mathcal{D}_{6}^{\tau}$ of asymptotic expansions as $x \rightarrow \infty$.

In Chapter 3 we are looking for asymptotic expansions of all five types (power, powerlogarithmic, complicated, semiexotic, and exotic) for the sixth Painlevé equation with 


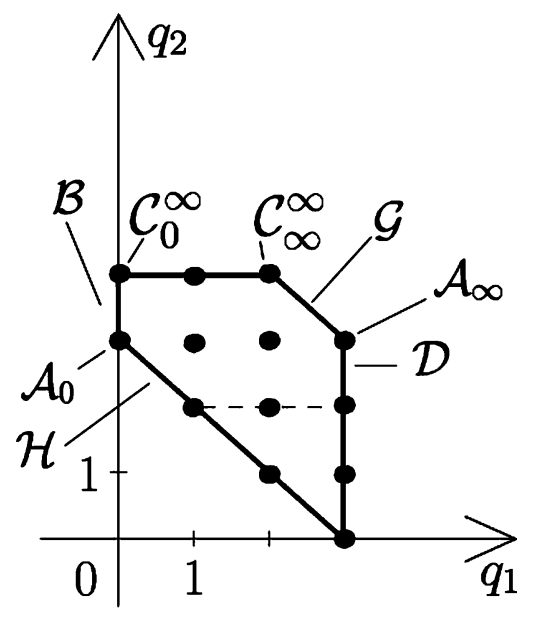

Figure 2. The polygon of the equation (9) and notation for families of expansions corresponding to vertices and edges of this polygon for $a=0$, $b \neq 0$.

$a=0, b \neq 0$ and $a \neq 0, b=0$. In $\oint$ in each of the cases $a=0, b \neq 0$ and $a \neq 0, b=0$ we discuss properties of the equation related to the polygon and symmetries. For example, in the case $a=0, b \neq 0$ the polygon of the equation is shown in Figure 2 ,

In $\S 2$ we consider the case $a=0, b \neq 0$ and study asymptotic expansions of solutions near zero (different from those in the case $a \cdot b \neq 0$ ) corresponding to the vertex $(0,4)$. The main result is as follows.

Theorem 3.2.1. For $a=0, c \neq 0$ there exists a one-parameter family $\mathcal{C}_{0}^{\infty}$ of power expansions (as $x \rightarrow 0$ ) of solutions determined by the formula

$$
\mathcal{C}_{0}^{\infty}: \quad y=c_{r} x^{r}+\sum_{s} c_{s} x^{s}
$$

where $r=\sqrt{2 c}$ with $\operatorname{Re} \sqrt{2 c}<0, s$ runs over the set $\{r-l r+m ; l, m \geq 0 ; l+m>0$; $l, m \in \mathbb{Z}\}, c_{r}$ is an arbitrary nonzero complex constant, and all $c_{s}$ are uniquely determined constants.

The family $\mathcal{C}_{0}^{\infty}$ exhausts all expansions corresponding to the vertex $(0,4)$ in Figure 2 . In $\oint 3$ we show that for $a=0, b \neq 0$ there are no asymptotic expansions near zero corresponding to the horizontal edge in Figure 2, In $\S 4$ we study the case $a=0, b \neq 0$ and consider asymptotic expansions near zero different from expansions corresponding to the vertical edge and different from the expansions that are present in the case $a \cdot b \neq 0$. Here the main result is as follows.

Theorem 3.4.1. (a) For $x \rightarrow 0$ and $a=0$ there exist two two-parameter (in $C_{11}$ and $\rho)$ families of exotic expansions of solutions with constant complex coefficients

$$
\mathcal{B}_{7}^{\tau}: \quad y=\frac{\rho^{2}}{2 c-\rho^{2}} \frac{1}{\sin ^{2}\left[\ln \left(C_{11} x\right)^{\gamma}\right]}+\sum_{\operatorname{Re} s \geq 1} c_{s} x^{s},
$$

where $\rho$ is an arbitrary purely imaginary constant, $\rho^{2} \neq 2 c, \tau=\operatorname{sgn}(\operatorname{Im} \rho), s \in\{\rho+l \rho+$ $m(1-\rho), l, m \geq 0 ; l+m>0 ; l, m \in \mathbb{Z}\}, 2 \gamma=i \rho, C_{11}$ is an arbitrary nonzero constant, and $c_{s}$ are uniquely determined constants. The families of inverse expansions $y^{-1}(x)$ for families $\mathcal{B}_{7}^{+}$and $\mathcal{B}_{7}^{-}$coincide. 
TABLE 1. Dependence of the existence of basic families $\mathcal{B}$ and $\mathcal{C}$ on the values of parameters $a$ and $c$.

\begin{tabular}{|c|c|c|c|c|}
\hline $0 \neq a \neq c \neq 0$ & $a=c \neq 0$ & $a \neq 0=c$ & $a=0 \neq c$ & $a=c=0$ \\
\hline $\mathcal{B}_{0}^{\tau}$ & $\mathcal{B}_{0}^{\tau}$ & $\mathcal{B}_{0}^{\tau}$ & $\mathcal{B}_{7}^{\tau}$ & $\mathcal{B}_{7}^{\tau}$ \\
\hline $\mathcal{B}_{1}, \mathcal{B}_{1}^{\tau}$ & $\mathcal{B}_{4}$ & $\mathcal{I}_{2}$ & $\mathcal{C}_{0}^{\infty}, \mathcal{I}_{4}$ & $\mathcal{I}_{2}$ \\
\hline $\mathcal{B}_{2}, \mathcal{B}_{2}^{\tau}$ & $\mathcal{B}_{2}, \mathcal{B}_{2}^{\tau}$ & $\mathcal{B}_{6}, \mathcal{B}_{6}^{\tau}$ & $\mathcal{B}_{8}, \mathcal{B}_{9}$ & $\mathcal{I}_{4}$ \\
\hline $\mathcal{B}_{3}$ & $\mathcal{B}_{5}$ & $\mathcal{B}_{3}$ & $\mathcal{B}_{3}$ & $\mathcal{B}_{10}$ \\
\hline
\end{tabular}

(b) For $x \rightarrow 0$ and $a=0, c \neq 0$ there exist two one-parameter families of power expansions of solutions:

(3.4.6)

$$
\mathcal{B}_{8,9}: \quad y=c_{\rho} x^{\rho}+\sum_{\operatorname{Re} s \geq 1} c_{s} x^{s}
$$

where $\rho$ is a purely imaginary constant, $\rho^{2}=2 c, \operatorname{Im} \rho>0$ for the family $\mathcal{B}_{8}$ and $\operatorname{Im} \rho<0$ for the family $\mathcal{B}_{9}, s \in\{\rho+l(1-\rho)+m, l, m \geq 0 ; l+m>0 ; l, m \in \mathbb{Z}\}$, and the complex coefficients are as follows: $c_{\rho}$ is an arbitrary nonzero constant and $c_{s}$ are uniquely determined constants.

(c) For $x \rightarrow 0$ and $a=c=0$ there exists a one-parameter family of expansions with constant complex coefficients

$$
\mathcal{B}_{10}: \quad y=c_{0}+\sum_{s=1}^{\infty} c_{s} x^{s}
$$

where $c_{0} \neq 0,1$ is an arbitrary constant and $c_{s}$ are uniquely determined constants.

For $a=0 \neq c$ there exists a one-parameter family of complicated expansions $\mathcal{B}_{3}$. The families $\mathcal{B}_{3}, \mathcal{B}_{8}-\mathcal{B}_{10}, \mathcal{B}_{7}^{\tau}$ exhaust all expansions corresponding to the left vertical edge in Figure 2. The series (3.2.8) and (3.4.22) converge for sufficiently small $|x|$. The $\mathcal{C}_{0}^{\infty}$ for integer values of $r$ and the family $\mathcal{B}_{10}$ were known earlier [54].

The families $\mathcal{C}_{0}^{\infty}, \mathcal{B}_{8}-\mathcal{B}_{10}, \mathcal{B}_{7}^{\tau}$ are also basic. Using symmetries of equation (2) the 21 basic families $\mathcal{A}_{0}, \mathcal{C}_{0}^{\infty}, \mathcal{B}_{1}-\mathcal{B}_{6}, \mathcal{B}_{8}-\mathcal{B}_{10}, \mathcal{B}_{0}^{\tau}-\mathcal{B}_{2}^{\tau}, \mathcal{B}_{6}^{\tau}, \mathcal{B}_{7}^{\tau}$ yield all other families of expansions near all three singular points. Near each point we have $20 \cdot 2+1=41$ families of expansions, this resulting in $3 \cdot 41=123$ families. However, among these expansions there are three occurrences (near $x=0,1, \infty$ ) of families of inverse expansions $y^{-1}(x)$ to expansions $\mathcal{B}_{0}^{\tau}$ and $\mathcal{B}_{7}^{\tau}$. According to Theorems 2.3.1.(a) and 3.4.1(a) these inverse expansions form one family for each of two values of $\tau$. Therefore, the number of different families decreases by 6 , i.e., becomes 117 .

Table 1 shows how the existence of the basic families $\mathcal{C}_{0}^{\infty}, \mathcal{B}_{1}-\mathcal{B}_{6}, \mathcal{B}_{8}-\mathcal{B}_{10}, \mathcal{B}_{0}^{\tau}-\mathcal{B}_{2}^{\tau}$, $\mathcal{B}_{6}^{\tau}-\mathcal{B}_{7}^{\tau}$ depends on the parameters of the equation. In $\S[$ we consider the case $a=0$, $b \neq 0$ and apply the symmetries to the basic families $\mathcal{C}_{0}^{\infty}, \mathcal{B}_{1}-\mathcal{B}_{6}, \mathcal{B}_{8}-\mathcal{B}_{10}, \mathcal{B}_{0}^{\tau}-\mathcal{B}_{2}^{\tau}$, $\mathcal{B}_{6}^{\tau}-\mathcal{B}_{7}^{\tau}$ obtaining the families $\mathcal{C}_{\infty}^{\infty}, \mathcal{G}_{1}-\mathcal{G}_{6}, \mathcal{G}_{8}-\mathcal{G}_{10}, \mathcal{G}_{0}^{\tau}-\mathcal{G}_{2}^{\tau}, \mathcal{G}_{6}^{\tau}-\mathcal{G}_{7}^{\tau}$ of asymptotic expansions of solutions near infinity (different from families that exist in the case $a \cdot b \neq 0$ ) that correspond to a vertex or to the inclined edge. In $\S 6$ we summarize and discuss the results obtained for the case $a=0, b \neq 0$. In $\S 7$ we apply one of the symmetries of equation (2) to obtain expansions of the solution in the case $b=0, a \neq 0$.

In Chapter 4 we consider the case $a=b=0$ of the sixth Painlevé equation. It does not contain any new results. In $\S 1$ we list asymptotic expansions that survive from the cases $a=0, b \neq 0$ and $a \neq 0, b=0$. In $\S 2$ we present examples, compare our results with those of Mazzocco [63, and point out to an error in Mazzocco's paper.

In Chapter 5 we use symmetries of equation P6 to obtain, starting from asymptotic expansions in a neighborhood of zero, asymptotic expansions in a neighborhood of 1 . 
Here we obtain all "flat" asymptotic expansions of solutions of equation (9) because either each "flat" reduced equation is solved explicitly, or one can prove that it has no appropriate solutions; this yields all possible asymptotics of solutions that can be obtained using methods of two-dimensional power geometry. Each asymptotics is expanded in all possible ways.

Let us present a brief survey of previously known results and compare them with the results of the present paper formulated above. In 1982 Shimomura 74 proved the existence and convergence of expansions from the family $\mathcal{A}_{0}$ in sectors of the complex plane, and Jimbo 61 deduced formulas relating the families $\mathcal{A}_{0}, \mathcal{A}_{1}$ and $\mathcal{A}_{\infty}$ for generic values of the parameters $a, b, c, d$. In 2000, Dubrovin and Mazzocco [46] considered the case $b=c=d-\frac{1}{2}=0, a=\frac{(2 \mu-1)^{2}}{2}, 2 \mu \notin \mathbb{Z}$ and found relations for the family $\mathcal{A}_{0}$. In 2001, Mazzocco [63] considered the case $b=c=d-\frac{1}{2}=0, a=\frac{(2 \mu-1)^{2}}{2}, 2 \mu \in \mathbb{Z}$ and found the family $\mathcal{A}_{0}$ and asymptotics for a part of the families $\mathcal{B}_{0}^{\tau}$ (as Picard solutions), as well as logarithmic asymptotic behavior of the complicated family $\mathcal{B}_{3}$ (as Chazy solutions). For more details see $\S 2$ in Chapter 4 . For these asymptotics she indicated the relation formulas. In 2001, Guzzetti [55] considered the case $b=c=d-\frac{1}{2}=0, a \in \mathbb{C}$. He introduced his "elliptic representation" of solutions of P6 and showed that all previous results by Dubrovin and Mazzocco can be obtained using elliptic representation. In 2002 Guzzetti [56] used elliptic representation for all values of the parameters $a, b, c, d$ to obtain asymptotics of the families $\mathcal{A}_{0}$ and $\mathcal{H}_{0}^{\tau}$ and the corresponding relation formulas. In 2006 Guzzetti [57] showed that all previous results of Shimomura, Jimbo, Dubrovin, and Mazzocco can be obtained using elliptic representation. In [58, Theorem 1] he found all solutions that can be expanded in a Taylor series. In our notation they are contained in families $\mathcal{B}_{1}, \mathcal{B}_{2}, \mathcal{B}_{6}$, and $\mathcal{B}_{10}$ for special relations between parameters of the equations and for zero values of some arbitrary constants.

In 2008 Guzzetti [59] found logarithmic asymptotics from the families $\mathcal{H}_{3}, \mathcal{H}_{4}$ and $\mathcal{H}_{5}$ and the relation formulas for these asymptotics.

Some of the results of this paper were announced in [12, 14, 15, 25, 30] and in abstracts [28, 29, 31, 32, 41, 52, 53. Detailed exposition of the main results of the paper can be found in preprints [16]-[20] and [26, 33]. In the present paper the decomposition into families, the number of families, and the notation differ from those in previous publications since here we use a different definition of an exotic expansion (following preprint [11; here it is $\S 5$ in Chapter 1). In particular, now all exotic expansions are related to edges, whereas in earlier papers some of them were related to vertices.

The numbering of lemmas, theorems, corollaries, remarks, and formulas renews in each chapter: the first component indicates the chapter, the second the section. Tables and figures are numbered consecutively throughout the paper.

\section{Chapter 1. Computation of asymptotic expansions For solutions OF ORDINARY DIFFERENTIAL EQUATIONS}

In this chapter we present those methods and results of the two-dimensional power geometry [2] - 27] and [4] that are used in this paper. Power geometry methods can be applied to a rather wide class of equations and systems, of which the Painlevé equations represent a rather narrow subclass with their own special properties. These special properties imply, in particular, that power asymptotic expansions of solutions have at most one critical number and the expansion does not contain iterated logarithms. Therefore, in this chapter we present only the results related to the case where there is at most one critical number and only simple logarithm. 


\section{Power Asymptotics of SOlutions}

1.1. Main definitions and formulations of the problem. Let $x$ be an independent variable and $y$ a function of $x$, where $x, y \in \mathbb{C}$. Denote $X=(x, y)$.

$A$ differential monomial $a(X)$ is the product of a usual monomial

$$
c x^{r_{1}} y^{r_{2}}
$$

where $c=$ const $\in \mathbb{C}, R=\left(r_{1}, r_{2}\right) \in \mathbb{R}^{2}$, and a finite number of derivatives of the form

$$
\frac{d^{l} y}{d x^{l}}, \quad l \in \mathbb{N}
$$

A sum of differential monomials

$$
f(X)=\sum a_{i}(X)
$$

is called a differential sum.

Suppose we are given an ordinary differential equation

$$
f(X)=0,
$$

where $f(X)$ is a differential sum containing only integral powers of $y$. We set

$$
\omega=\left\{\begin{array}{rll}
-1 & \text { if } & x \rightarrow 0 \\
1 & \text { if } & x \rightarrow \infty
\end{array}\right.
$$

Let $x \rightarrow 0$ or $x \rightarrow \infty$ and a solution of equation (1.1.4) have the form

$$
y=c_{r} x^{r}+o\left(|x|^{r+\varepsilon}\right),
$$

where $c_{r}=$ const $\in \mathbb{C}, c_{r} \neq 0, r, \varepsilon \in \mathbb{R}$ and $\omega \varepsilon<0$. Then the expression

$$
y=c_{r} x^{r}, \quad c_{r} \neq 0
$$

is a power asymptotics of equation (1.1.6).

Problem 1.1. For a given equation (1.1.4) find all power asymptotics (1.1.7) of its solutions of the form (1.1.6).

Power geometry provides the theory and algorithms for solving Problem 1.1 based on the extraction of reduced equations.

1.2. Extraction of reduced equations. With each differential monomial $a(X)$ we associate its (vector) exponent $Q(a)=\left(q_{1}, q_{2}\right) \in \mathbb{R}^{2}$ defined by the following rules. For a monomial of the form (1.1.1) we set $Q\left(c X^{R}\right)=R$, i.e., $Q\left(c x^{r_{1}} y^{r_{2}}\right)=\left(r_{1}, r_{2}\right)$; for the derivative (1.1.2) we set $Q\left(d^{l} y / d x^{l}\right)=(-l, 1)$; the exponent of a product of differential monomials is the (vector) sum of the exponents of factors: $Q\left(a_{1} a_{2}\right)=Q\left(a_{1}\right)+Q\left(a_{2}\right)$. The set $\mathbf{S}(f)$ of exponents $Q\left(a_{i}\right)$ of all differential monomials $a_{i}(X)$ in a differential sum (1.1.3) is called the support of the sum $f(X)$. Clearly, $\mathbf{S}(f) \in \mathbb{R}^{2}$. By $f_{Q}(X)$ we denote the sum of those monomials $a_{i}(X)$ in (1.1.3) that satisfy $Q\left(a_{i}\right)=Q$. Then the differential sum (1.1.3) can be written in the form

$$
f(X)=\sum f_{Q}(X) \quad(\text { sum over } Q \in \mathbf{S}(f)) .
$$

The closure of the convex hull $\Gamma(f)$ of the support $\mathbf{S}(f)$ is called the polygon of the sum $f(X)$. The boundary $\partial \Gamma(f)$ of the polygon $\Gamma(f)$ consists of vertices $\Gamma_{j}^{(0)}$ and edges $\Gamma_{j}^{(1)}$. Together, they are called generalized faces $\Gamma_{j}^{(d)}$; here the superscript indicates the dimension of the face, and the subscript indicates its index. To each face $\Gamma_{j}^{(d)}$ we associate the reduced sum

$$
\hat{f}_{j}^{(d)}(X)=\sum a_{i}(X) \quad\left(\text { the sum over } Q\left(a_{i}\right) \in \mathbf{S}(f) \cap \Gamma_{j}^{(d)}\right) .
$$


Let the plane $\mathbb{R}_{*}^{2}$ be dual to the plane $\mathbb{R}^{2}$, so that for $P=\left(p_{1}, p_{2}\right) \in \mathbb{R}_{*}^{2}$ and $Q=$ $\left(q_{1}, q_{2}\right) \in \mathbb{R}^{2}$ we have the inner product $\langle P, Q\rangle \stackrel{\text { def }}{=} p_{1} q_{1}+p_{2} q_{2}$. To each face $\Gamma_{j}^{(d)}$ in the plane $\mathbb{R}_{*}^{2}$ one associates the corresponding normal cone

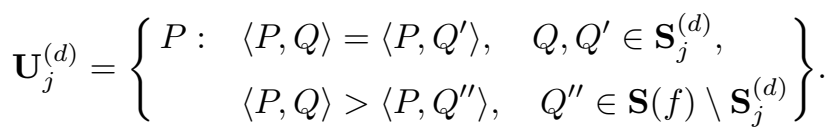

Let the vector $N_{j}$ be the outer normal to the edge $\Gamma_{j}^{(d)}$ (vectors are denoted by capital letters). The normal cone of an edge $\Gamma_{j}^{(1)}$ is the ray generated by the vector $N_{j}$ and directed outside of the polygon $\Gamma(f)$. For a vertex $\Gamma_{j}^{(0)}$ the normal cone $\mathbf{U}_{j}^{(0)}$ is an open sector (angle) on the plane $\mathbb{R}_{*}^{2}$ with the vertex at the origin $P=0$ bounded by the rays that are normal cones of the edges adjacent to the vertex $\Gamma_{j}^{(0)}$.

So, to each face $\Gamma_{j}^{(d)}$ we have associated the normal cone $\mathbf{U}_{j}^{(d)}$ in $\mathbb{R}_{*}^{2}$ and the reduced equation

$$
\hat{f}_{j}^{(d)}(X)=0 .
$$

Theorem 1.1.1 (3, Ch. VI, Theorem 1.1]). Equation (1.1.4) has a solution (1.1.6), and $\omega(1, r) \in \mathbf{U}_{j}^{(d)}$, the reduction (1.1.7) of the solution (1.1.6), is a solution of the reduced equation (1.1.9).

Therefore, to find all reduced solutions (1.1.7) of equation (1.1.4) one should compute the following data: the support $\mathbf{S}(f)$, the polygon $\Gamma(f)$, all its faces $\Gamma_{j}^{(d)}$, normal cones of edges $\mathbf{U}_{j}^{(1)}$, and normal cones of vertices $\mathbf{U}_{j}^{(0)}$. Then for each rescued equation (1.1.8), (1.1.9) one should find all solutions (1.1.7) for which one of the vectors $\pm(1, r)$ lies in the normal cone $\mathbf{U}_{j}^{(d)}$. For $d=0$ this means that one of the vectors $\pm(1, r)$ lies in $\mathbf{U}_{j}^{(d)}$. For $d=1$ this property always holds.

1.3. Solving the reduced equation. We consider separately the case of a vertex $\Gamma_{j}^{(0)}$ and of an edge $\Gamma_{j}^{(1)}$. To a vertex $\Gamma_{j}^{(0)}=\{Q\}$ there corresponds a reduced equation (1.1.9) with $d=0$ whose support consists of one point $Q$. Set $g(X) \stackrel{\text { def }}{=} X^{-Q} \hat{f}_{j}^{(0)}(X)$. Then solution (1.1.7) of equation (1.1.9) satisfies the equation $g(X)=0$. Substituting $y=c x^{r}$ in $g(X)$ we obtain that $g\left(x, c x^{r}\right)$ does not depend on $x$ and $c$ and is a polynomial in $r$, i.e., $g\left(x, c x^{r}\right) \equiv \chi(r)$, where $\chi(r)$ is the characteristic polynomial of the differential sum $\hat{f}_{j}^{(0)}(X)$. Therefore, for a solution (1.1.7) of equation (1.1.9) the exponent $r$ is a root of the characteristic equation

$$
\chi(r) \stackrel{\text { def }}{=} g\left(x, x^{r}\right)=0,
$$

and the coefficient $c_{r}$ is arbitrary. Among the roots $r_{i}$ of equation (1.1.10) one should choose those for which one of the vectors $\omega(1, r)$, where $\omega= \pm 1$, is in the normal cone $\mathbf{U}_{j}^{(0)}$ of the vertex $\Gamma_{j}^{(0)}$. Here the value of $\omega$ is determined uniquely. The corresponding expressions (1.1.7) with an arbitrary constant $c_{r}$ are candidates to being reduced solutions of equation (1.1.4).

A reduced equation (1.1.9) is called algebraic if it does not contain derivatives.

Remark 1.1.1 ([3]). If the reduced equation (1.1.9) with $d=0$ is algebraic, then it has no solutions of the form (1.1.7). Therefore we may disregard reductions consisting of one algebraic monomial. 
To an edge $\Gamma_{j}^{(1)}$ there corresponds the reduced equation (1.1.9) with $d=1$ such that its normal cone is the ray $\mathbf{U}_{j}^{(1)}$ in $\left\{\lambda N_{j}, \lambda>0\right\}$. The inclusion $\omega(1, r) \in \mathbf{U}_{j}^{(1)}$ uniquely determines the exponent $r$ of the reduced equation (1.1.7) and the value $\omega= \pm 1$ in (1.1.5). To determine the coefficient $c_{r}$ one should substitute (1.1.7) in the reduced equation (1.1.9). After canceling a power of $x$ we obtain the algebraic determining equation for the coefficient $c_{r}$ :

$$
\tilde{f}\left(c_{r}\right) \stackrel{\text { def }}{=} x^{-s} \hat{f}_{j}^{(1)}\left(x, c_{r} x^{r}\right)=0 .
$$

To each root $c_{r}=c_{r i} \neq 0$ of this equation there corresponds the expression (1.1.7) that is a candidate to the role of the reduced solution of equation (1.1.4). According to (1.1.5), if in the normal cone $\mathbf{U}_{j}^{(1)}$ we have $p_{1}<0$, then $x \rightarrow 0$, and if $p_{1}>0$, then $x \rightarrow \infty$.

So each reduced equation (1.1.9) has several appropriate solutions (1.1.7) with $\omega(1, r)$ $\subset \mathbf{U}_{j}^{(d)}$. Let us combine them in families that are continuous in $\omega, r, c_{r}$, and parameters of the equation (1.1.4).

If we are interested not in all solutions (1.1.6) of equation (1.1.4) but only those for which $\omega(1, r)$ is in some given cone $\mathcal{K}$, then $\mathcal{K}$ is called the cone of the problem 3 , Chapter I, $\S 6$ ]. For example, for the reduced equation (1.1.9) the cone of the problem is the normal cone $\mathbf{U}_{j}^{(d)}$ provided that there are no other restrictions.

1.4. Critical numbers of the reduced solution. If the reduced solution (1.1.7) is found, then the change of variables

$$
y=c_{r} x^{r}+z
$$

transforms (1.1.4) to the form

$$
\tilde{f}(x, z) \stackrel{\text { def }}{=} f\left(x, c x^{r}+z\right)=0,
$$

where $\tilde{f}(x, z)$ is a differential sum such that all points $Q=\left(q_{1}, q_{2}\right)$ in its support $\mathbf{S}(\tilde{f})$ have an integral nonnegative coordinate $q_{2}$. To equation (1.1.13) one can apply the computations described above (i.e., to compute the support, the polygon, the reductions, etc.) and obtain for (1.1.6) the next term of the expansion $c_{k_{0}} x^{k_{0}}$ with $k_{0}>r$ if $x \rightarrow 0$ and $k_{0}<r$ if $x \rightarrow \infty$. Hence, we have Problem 1.1 for equation (1.1.13) with the cone of the problem now being

$$
\mathcal{K}=\left\{k=\frac{p_{2}}{p_{1}}: k \omega<r \omega, p_{1} \omega>0\right\} .
$$

However, in many cases the differential sum $\tilde{f}(x, z)$ has a special form, thus allowing us to simplify the computation of expansions (1.1.6). Let us assume that equation (1.1.13) has the form

$$
\tilde{f}(x, z) \stackrel{\text { def }}{=} \mathcal{L}(x) z+h(x, z)=0,
$$

where $\mathcal{L}(x)$ is a linear differential operator and the support $\mathbf{S}(\mathcal{L} z)$ consists of one point $(v, 1)$ that is a vertex $\tilde{\Gamma}_{1}^{(0)}$ of the polygon $\Gamma(\tilde{f})$. Then for all points $Q=\left(q_{1}, q_{2}\right)$ in the support $\mathbf{S}(h)$, the coordinate $q_{2}$ is nonnegative, and there is no point $Q=(v, 1)$ such that the normal cone of the vertex $\tilde{\Gamma}_{1}^{(0)}$ contains a vector $P=\left(p_{1}, p_{2}\right)$ with $p_{1} \omega>0$.

Similarly to the well-known in functional analysis Fréchet derivative [40, we introduce the formal Fréchet derivative (or the first variation) $\frac{\delta f(x, y)}{\delta y}$ of the differential sum $f(x, y)$ 
that has the following properties (and is uniquely determined by these properties):

$$
\begin{gathered}
\frac{\delta\left(c x^{q_{1}} y^{q_{2}}\right)}{\delta y}=c q_{2} x^{q_{1}} y^{q_{2}-1}, \quad \frac{\delta\left(d^{l} y / d x^{l}\right)}{\delta y}=\frac{d^{l}}{d x^{l}}, \\
\frac{\delta(f+g)}{\delta y}=\frac{\delta f}{\delta y}+\frac{\delta g}{\delta y}, \quad \frac{\delta(f g)}{\delta y}=\left(\frac{\delta f}{\delta y}\right) g+f\left(\frac{\delta g}{\delta y}\right) .
\end{gathered}
$$

According to the second property, the first variation is a linear functional operator, i.e., an operator of the form

$$
\sum_{k=0}^{l} g_{k}(x, y) \frac{d^{k}}{d x^{k}},
$$

where $g_{k}(x, y)$ are differential sums.

Theorem 1.1.2 (44, 4]). Let (1.1.7) be a solution of the reduced equation (1.1.9) with $\omega(1, r) \in \mathbf{U}_{j}^{(d)}$. Then for equation (1.1.15) we have

$$
\mathcal{L}(x)=\frac{\delta \hat{f}_{j}^{(d)}(x, y)}{\delta y} \quad \text { for } y=c_{r} x^{r}
$$

i.e., the operator $\mathcal{L}(x)$ is equal to the first variation computed at the solution (1.1.7). Furthermore, $\mathbf{S}(\mathcal{L} z)=(v, 1)$, where $v=\left\langle Q_{1},(1, r)\right\rangle-r$ with $Q_{1} \in \Gamma_{j}^{(d)}$.

Therefore, substitution (1.1.13) transforms equation (1.1.4) to the form (1.1.15) provided

$$
\mathcal{L}(x) \not \equiv 0 .
$$

Let $\nu(k)$ be the characteristic equation of the differential sum $\mathcal{L}(x) z$, i.e.,

$$
\nu(k)=x^{-v-k} \mathcal{L}(x) x^{k} .
$$

If $\nu(k) \not \equiv 0$, then the roots $k_{1}, \ldots, k_{s}$ of the polynomial $\nu(k)$ are called eigenvalues of the reduced solution (1.1.7). Those real eigenvalues $k_{i}$ that lie in the cone of the problem, i.e., satisfy inequalities (1.1.14), are called critical numbers. As will be shown in $\S 2$, they play an important role in finding expansions of the solution (1.1.6).

Remark 1.1.2. The power solution (1.1.7) of the algebraic reduced equation (1.1.9) with $d=1$ does not have eigenvalues and critical numbers since for such a solution,

$$
\nu(k) \equiv \nu_{0}=\text { const }=\frac{\partial \hat{f}_{j}^{(1)}}{\partial y}\left(1, c_{r}\right) .
$$

If $c_{r}$ is a simple root of equation (1.1.11), then $\nu_{0} \neq 0$. If $c_{r}$ is a multiple root of equation (1.1.11), then $\nu_{0}=0$.

If $\mathcal{L}(x) \not \equiv 0$, then $\nu(k) \not \equiv 0$. If $\mathcal{L}(x) \equiv 0$, then one should compute the solution of equation (1.1.13) (taking into account the cone of the problem (1.1.14) ) as described in $\S \$ 1.2,1.3$

1.5. Asymptotics with complex exponents. Consider solutions of the form

$$
y=c_{\rho} x^{\rho}+o\left(|x|^{r+\varepsilon}\right), \quad c_{\rho} \neq 0
$$

with a complex exponent $\rho=r+i s$, where $\varepsilon \omega<0$. The asymptotics of such a solution is of the form

$$
y=c_{\rho} x^{\rho}, \quad c_{\rho} \neq 0 .
$$

All the facts from previous subsections are true for such solutions as well if we set $r=\operatorname{Re} \rho$. 
We only mention that solutions of the form (1.1.20) of the reduced equation (1.1.9) occur only for vertices $\Gamma_{j}^{(0)}$, i.e., only in the case $d=0$. In this case the characteristic equation (1.1.10) can have complex roots, which we will denote $\rho_{1}, \ldots, \rho_{l}$. A root $\rho_{i}$ is appropriate if one of the vectors $\pm\left(1, \operatorname{Re} \rho_{i}\right)$ is in the normal cone $\mathbf{U}_{j}^{(0)}$. If, instead of (1.1.12), we make the change

$$
y=c_{\rho} x^{\rho}+z,
$$

then the differential sum $\tilde{f}(x, z) \stackrel{\text { def }}{=} f\left(x, c_{\rho} x^{\rho}+z\right)$ will contain the usual monomials of the form $c x^{r_{1}} z^{r_{2}}$ with complex $r_{1}$. Theorem 1.1.2 remains valid provided we compute the first variation on the solution (1.1.20). The characteristic polynomial $\nu(k)$ is again defined by (1.1.18). Its roots $k_{i}$ again are eigenvalues of the reduced solution (1.1.20). Those roots $k_{i}$ for which $\operatorname{Re} k_{i}$ is in the cone of the problem, i.e., satisfies inequalities (1.1.14), are called critical numbers of the reduced solution (1.1.20).

For the reduced equation (1.1.9) corresponding to an edge $\Gamma_{j}^{(1)}$ we are looking for solutions (1.1.7) with real exponent $r$; this exponent is determined uniquely by the normal vector to the edge. Solutions of the form (1.1.20) with complex $\rho, \operatorname{Re} \rho=r, \operatorname{Im} \rho \neq 0$ will be considered later in $\S 5$.

In what follows we restrict ourselves to the case where there is at most one critical number.

\section{Power AND POWER-LOgARITHMiC EXPANSIONS}

2.1. Formulation of the problem. Consider equation (1.1.15) with $\nu(k) \not \equiv 0$. When looking for power series solutions of this equation,

$$
z=\sum c_{k} x^{k}, \quad \omega k<\omega r
$$

with $c_{k}=$ const $\in \mathbb{C}$, we see that according to 4 such an expansion exists only under certain conditions, with the main condition being the absence of critical values. In the case where we do not impose these conditions, we get expansions of the form (1.2.1), where the $c_{k}$ are polynomials in $\ln x$.

Consider the equation (1.1.15), i.e.,

$$
\tilde{f}(x, z) \stackrel{\text { def }}{=} \mathcal{L}(x) z+h(x, z)=0
$$

where $\tilde{f}(x, z)$ is a differential sum with $z$ entering in integer nonnegative powers and $\mathcal{L}(x)$ is a linear differential operator.

Problem 2.1. For the solutions of the equation (1.2.2), find all expansions of the form

$$
z=\sum \beta_{k}(\ln x) x^{k}
$$

where $\beta_{k}$ are polynomials in $\ln x$ with complex coefficients and exponents $k$ or Re $k$ lie in the cone of the problems (1.1.14) provided it exists.

2.2. The support of the expansion of a solution. To be specific, we will impose the following conditions to the summands in equation (1.2.2).

Condition 2.1. The point $(v, 1)$ is a vertex of the polygon $\Gamma(\tilde{f})$. The only corresponding term in the sum $\tilde{f}(x, z)$ is $\mathcal{L}(x) z$.

If (1.2.2) is obtained from (1.1.4) and $\mathcal{L}(x) \not \equiv 0$, this condition is satisfied automatically. If this condition is satisfied, then the differential sum $\mathcal{L}(x) z$ has the characteristic polynomial (1.1.18) and $\nu(k) \not \equiv 0$.

Let us shift the support $\mathbf{S}(\tilde{f})$ by the vector $(-v,-1)$. Then the vertex $(v, 1)$ corresponding to the term $\mathcal{L}(x) z$ moves to the origin. Let a number $r$ be such that for each 
point $Q^{\prime} \in \mathbf{S}^{\prime} \stackrel{\text { def }}{=} \mathbf{S}(\tilde{f})-(v, 1)$ we have $\left\langle\omega R, Q^{\prime}\right\rangle \geq 0$, where $R=(1, r)$. Let $k_{1}$ be a real number satisfying the inequality $k_{1} \omega<r \omega$ in (1.1.14). Finally, let $\mathbf{S}_{+}^{\prime}\left(k_{1}\right)$ be the set consisting of finite sums of vectors $Q^{\prime} \in \mathbf{S}^{\prime}$ and the vector $\left(k_{1},-1\right)$. Denote

$$
\mathbf{K}\left(k_{1}\right)=\mathbf{S}_{+}^{\prime}\left(k_{1}\right) \cap\left\{q_{2}=-1\right\} .
$$

Proposition 1.2.1 ([8]). If the support $\mathbf{S}(\tilde{f})$ does not have accumulation points in $\mathbb{R}^{2}$, then the set $\mathbf{K}\left(k_{1}\right)$ does not have accumulation points in $\mathbb{R}$.

Proposition 1.2.2 ([8]). Let $\Gamma_{j}^{(0)}$ be a vertex of the polygon $\Gamma(f)$ of the equation (1.1.4) such that the corresponding reduced equation (1.1.9) has a solution (1.1.7) with $\omega(1, r) \in \overline{\mathbf{U}}_{j}^{(0)}$ and all points of the shifted support $\mathbf{S}(f)-\Gamma_{j}^{(0)}$ are represented in the form $\sum_{i=1}^{n} l_{i} M_{i}$, with integers $l_{i} \geq 0$, and some vectors $M_{i} \in \mathbb{R}^{2}$. Then for the set $\mathbf{K}$ of equation (1.2.2) we have

$$
\mathbf{K} \subset\left\{k=r+\sum_{i=1}^{n} l_{i} t_{i}, \quad \text { integers } l_{i} \geq 0, \quad \sum_{i=1}^{n} l_{i}>0\right\},
$$

where $t_{i}=\left\langle(1, r), M_{i}\right\rangle, i=1, \ldots, n$. Here $\overline{\mathbf{U}}_{j}^{(0)}$ denotes the closure of the cone $\mathbf{U}_{j}^{(0)}$.

Proposition 1.2.3 ([8]). If the set $\mathbf{K}$ has the form

$$
\mathbf{K}=\left\{k=r+\sum_{i=1}^{n} l_{i} t_{i}, \quad \text { integers } l_{i} \geq 0, \quad \sum_{i=1}^{n} l_{i}>0\right\},
$$

then the set (1.2.4) has the form

$$
\mathbf{K}\left(k_{1}\right)=\left\{k=r+\sum_{i=1}^{n} l_{i} t_{i}+m\left(k_{1}-r\right), \quad \text { integers } l_{i}, m \geq 0, \quad \sum_{i=1}^{n} l_{i}+m>0\right\} .
$$

\subsection{Computation of expansions.}

Theorem 1.2.1 ([8]). If equation (1.2.2) satisfies Condition 2.1, then it has a formal solution

$$
z=z^{*}(x) \stackrel{\text { def }}{=} \sum \beta_{k}(\ln x) x^{k}, \quad k \in \mathbf{K}\left(k_{1}\right),
$$

where $\beta_{k}(\ln x)$ are polynomials in $\ln x$ and $k_{1}$ is a critical number of the reduced solution (1.1.7).

The set $\mathbf{K}\left(k_{1}\right)$ is the maximal possible (theoretical) support of expansion (1.2.6). The actual support is a subset of this set.

Indeed, moving along the points $k$ of the set (1.2.4) in the direction of increase of $-\omega(k-r)$, for each coefficient $\beta_{k}$ in (1.2.6) we get a linear equation

$$
\mathcal{L}(x) \beta_{k} x^{k}+\theta_{k} x^{k+v}=0,
$$

where $\theta_{k}$ is a polynomial in the coefficients $\beta_{j}$ and their derivatives with $-\omega(j-r)<$ $-\omega(k-r)$, i.e., $-\omega j<-\omega k$. Furthermore, the coefficient $\theta_{k}$ depends on the coefficients in the sum $h$ in (1.2.2). In fact, $\theta_{k}$ is the coefficient at $x^{k+v}$ in the sum

$$
h\left(x, \sum_{-\omega r<-\omega j<-\omega k} \beta_{j} x^{j}\right) .
$$

Let the conclusion of the theorem hold for all $\beta_{j}$ with $-\omega j<-\omega k$. Then $\theta_{k}$ is a polynomial in $\xi \stackrel{\text { def }}{=} \ln x$. 
Lemma 1.2.1 ([8]). Equation (1.2.7) is equivalent to the linear differential equation

$$
\mathcal{N}_{k}(\xi) \beta_{k}(\xi)+\theta_{k}(\xi) \stackrel{\text { def }}{=} \sum \frac{1}{m !} \nu^{(m)}(k) \beta_{k}^{(m)}(\xi)+\theta_{k}(\xi)=0,
$$

where $\nu^{(m)}(k)=\left.\frac{d^{m} \nu(q)}{d q^{m}}\right|_{q=k}, \quad \beta_{k}^{(m)}=\frac{d^{m} \beta_{k}(\xi)}{d \xi^{m}}, \quad m=0,1,2, \ldots$

Let $\mu(k)$ be the smallest $m$ such that $\nu^{(m)}(k) \neq 0$, and $\lambda(k)$ the degree of the polynomial $\theta_{k}(\xi)$; we agree that $\lambda(k)=-1$ if $\theta_{k} \equiv 0$.

Lemma 1.2.2 (8]). Let $\theta_{k}(\xi)$ be a polynomial of degree $\lambda(k)$. Then equation (1.2.9) has a solution $\beta_{k}(\xi)$ that is a polynomial of degree $\mu(k)+\lambda(k)$ and contains $\mu(k)$ arbitrary coefficients.

Remark 1.2.1. If we know the operator $\mathcal{L}(x)$ and the support $\mathbf{K}\left(k_{1}\right)$ of an expansion (1.2.6), we can compute the coefficients of this expansion using the initial equation $f(x, y)=0$; indeed, the coefficient at $x^{k}$ in the sum (1.2.8) coincides with the coefficient at $x^{k+v}$ in the sum

$$
f\left(x, c_{r} x^{r}+\sum_{-\omega r<-\omega j<-\omega k} \beta_{j} x^{j}\right) .
$$

Therefore, computing the initial terms of the expansion

$$
y=c_{r} x^{r}+\sum \beta_{j} x^{j}
$$

and substituting them in $f(x, y)$ we obtain the function $\theta_{k}$ in equation (1.2.9). In complicated cases this equation can be solved as explained in Lemma 1.2.2.

We say that a critical number $k_{1}$ satisfies the compatibility condition if for $k=k_{1}$ in equation (1.2.7) we have $\theta_{k} \equiv 0$.

Corollary 1.2.1. Let in equation (1.2.7) $\theta_{k}$ be a polynomial of degree $\lambda(k)$ in $\ln x$.

If in equation (1.2.7) the number $k$ is not critical and $\theta_{k}=$ const, then equation (1.2.7) can be reduced to the equation

$$
\nu(k) \beta_{k}+\theta_{k}=0,
$$

which has a solution $\beta_{k}=-\frac{\theta_{k}}{\nu(k)}$.

Similarly, if $\theta_{k}$ is a polynomial of degree $\lambda(k)$ in $\ln x$ and $\nu(k) \neq 0$, then there exists a solution of equation (1.2.7) such that $\beta_{k}$ is a polynomial in $\ln x$ of degree $\lambda(k)$.

If in equation (1.2.7) the number $k$ is the only simple (nonmultiple) critical number and $\theta_{k}=$ const, then we look for $\beta_{k}$ in the form $\beta_{k}=\alpha_{k}+\gamma_{k} \ln x$ and equation (1.2.7) takes the form

$$
\nu^{\prime}(k) \gamma_{k}+\theta_{k}=0
$$

This equation has a solution $\gamma_{k}=-\frac{\theta_{k}}{\nu^{\prime}(k)}$. Here $\alpha_{k}$ is an arbitrary number. If $\theta_{k}=0$ (i.e., the compatibility condition is satisfied), then $\gamma_{k}=0$ and $\beta_{k}=\alpha_{k}$ is an arbitrary constant. Therefore, in this case, logarithms do not occur.

Usually one can compute not the entire expansion (1.2.6) but just a number of its initial terms. It is usually desirable that these terms contain a critical value $k_{1}$. If this is the case, they contain all arbitrary constants of the expansion. 
2.4. Powers of logarithms in the expansion. It is clear that degree ord $\beta_{k}(\xi)$ of the polynomial $\beta_{k}$ increases as $-\omega(k-r)$ grows. Let us estimate this degree from above. Denote $æ(j)=\frac{\mu(j)}{|j-r|}$ and $q^{*}=\max q_{2}$ for $\left(q_{1}, q_{2}\right) \in \mathbf{S}(\tilde{f})$.

Theorem 1.2.2 ([8]). If the equation (1.2.2) contains no logarithms, then for the solution (1.2.6) we have

$$
\operatorname{ord} \beta_{k} \leq q^{*}|k-r| \sum_{0<-\omega(j-r)<-\omega(k-r)} x(j)
$$

where $j, k \in \mathbf{K}\left(k_{1}\right)$.

Remark 1.2.2. Under the conditions of Theorem 1.2.2 the decomposition (1.2.6) may contain the logarithm $\ln x$ only in two cases: a) if the set $\mathbf{K}$ contains a critical number $k_{1}$ that does not satisfy the compatibility condition; b) if $k_{1}$ is a multiple critical number (this does not occur in our case). This agrees with results in [4].

2.5. The lattice of the support of an expansion. A discrete subset $\mathbf{Z}$ in $\mathbb{R}^{n}$ is called a lattice if it is closed under vector addition and subtraction. Vectors $B_{1}, \ldots, B_{n}$ form a base of a lattice $\mathbf{Z}$ if each point $Q \in \mathbf{Z}$ can be represented in the form

$$
Q=m_{1} B_{1}+\cdots+m_{n} B_{n}, \quad m_{i} \in \mathbb{Z} .
$$

Theorem 1.2.3 (4 $)$. If the set $\mathbf{S}^{\prime} \stackrel{\text { def }}{=} \mathbf{S}(f)-(v, 1)$ and the point $\left(k_{1},-1\right)$ lie in a lattice $\mathbf{Z}$, then

$$
\mathbf{K}\left(k_{1}\right) \subset \mathbf{Z} \cap\left\{q_{2}=-1\right\} .
$$

2.6. Computation of the second approximation. Assume that we know the reduced equation (1.1.9), an appropriate solution (1.1.7), and the critical number $k_{1}$. Let us show how to find the point $s_{0} \in \mathbf{K}$ closest to $r$. Let the vector $P=\omega(1, r), \omega= \pm 1$ (see (1.1.5)), and $\mu^{\prime}=\max \left\langle P, Q^{\prime}\right\rangle$, where $Q \in \mathbf{S}_{j}^{(d)}, Q^{\prime} \in \mathbf{S}(f) \backslash \mathbf{S}_{j}^{(d)}$, and $\mathbf{S}_{j}^{(d)}=\mathbf{S}(f) \cap \Gamma_{j}^{(d)}$.

Theorem 1.2.4 (14]). The closest to $r$ point $s_{0}$ satisfies

$$
\left|s_{0}-r\right|=\mu-\mu^{\prime} .
$$

If the critical number $k_{1}$ does not exist or if it is such that $\left|k_{1}-r\right|>\mu-\mu^{\prime}$, we can compute the coefficient $c_{s_{0}}$ as follows. In the differential sum $f(x, y)$ we separate the second approximation

$$
\hat{\hat{f}}=\sum f_{Q}(x, y) \quad\left(\text { the sum over } Q:\langle P, Q\rangle=\mu^{\prime}\right),
$$

substitute $y=c_{r} x^{r}+c_{s_{0}} x^{s_{0}}$ in the sum

$$
\hat{f}_{j}^{(d)}+\hat{\hat{f}}
$$

and reduce similar terms. Making the coefficient at $x^{\mu^{\prime} \omega}$ in the sum (1.2.13) equal to zero, we obtain a linear equation $\nu\left(s_{0}\right) c_{s_{0}}+b_{s_{0}}=0$, where $b_{s_{0}}$ is the coefficient at $x^{\mu^{\prime} \omega}$ in the sum $\hat{\hat{f}}\left(x, c_{r} x^{r}\right)$.

If there exists a critical number $k_{1}$ such that $\left|k_{1}-r\right|<\mu-\mu^{\prime}$, then $s_{0}=k_{1}$ and the coefficient $c_{s_{0}}$ is arbitrary. 
2.7. Complex exponents. Let $k_{1}$ be a complex number such that its real part Re $k_{1}$ is in the cone of the problem (1.1.14), i.e., $\omega$ Re $k_{1}<\omega r$. In this case the set $\mathbf{S}_{+}^{\prime}\left(k_{1}\right)$ contains points $Q=\left(q_{1}, q_{2}\right)$ such that $q_{1} \in \mathbb{C}, q_{2} \in \mathbb{Z}$. Also let $\mathbf{K}$ be a subset of the complex plane $q_{1} \in \mathbb{C}$. Then Theorem 1.2.1 remains true, but under the expansion (1.2.6) exponents $k$ are partially ordered by the growth of $-\omega(\operatorname{Re} k-r)$. All other results of $\S \$ 2.22 .5$ also remain true.

If in equation (1.2.2) there is a term $x^{r_{1}}$ with a complex exponent $r_{1}$, then the support $\mathbf{S}(\tilde{f})$ lies in the direct sum $\mathbb{C} \oplus \mathbb{R}$. However, when constructing the polygon $\Gamma(\tilde{f})$ one should take into account only $\operatorname{Re} q_{1}$, i.e., $\operatorname{Re} \mathbf{S}(\tilde{f})$. All further constructions and results hold true. Expansions of solutions (1.2.1) with complex exponents $k$ were considered in [45].

\section{Nonpower ASYMptotics OF SOlutions}

3.1. Main definitions and formulation of the problem. Let $x \rightarrow 0$ or $x \rightarrow \infty$. Two functions $\varphi(x)$ and $\psi(x)$ are said to be weakly (asymptotically) equivalent if

$$
\frac{\psi(x)}{\varphi(x)} \rightarrow 1
$$

In this case the function $\varphi(x)$ is a weak asymptotics of the function $\psi(x)$ and vice versa. Denote by $\exp ^{(k)} x$ and $\ln ^{(k)} x$ the $k$-th iterate of the exponential function and of the logarithmic function, respectively, i.e.,

$$
\exp ^{(k)} x \stackrel{\text { def }}{=} \exp (\exp (\ldots\{\exp (x)\} \ldots)) \quad \text { and } \quad \ln ^{(k)} x \stackrel{\text { def }}{=} \ln (\ln (\ldots(\ln x) \ldots)) .
$$

Problem 3.1. For solutions of equation (1.1.4), where $f(x, y)$ is a differential sum, find all (weak) asymptotics of the form

$$
y=c x^{r}(\exp x)^{s_{1}} \ldots\left(\exp ^{(k)} x\right)^{s_{k}}(\ln x)^{t_{1}} \ldots\left(\ln ^{(l)} x\right)^{t_{l}},
$$

where $c=$ const $\in \mathbb{C}, c \neq 0 ; r, s_{i}, t_{i}=$ const $\in \mathbb{R}$ or $\mathbb{C}$.

In $\S 1$ we have presented a method of computing all power asymptotics, i.e., of asymptotics of the form (1.1.7). Therefore here we concentrate on the computation of all nonpower asymptotics, of solutions of (1.3.1), i.e., asymptotics of the form different from (1.1.7).

The order of a function $\varphi(x)$ is the number

$$
r=\lim \frac{\ln |\varphi(x)|}{\ln |x|} \in[-\infty,+\infty],
$$

provided the limit exists. It is clear that two weakly equivalent functions have the same order.

Two functions $\varphi(x)$ and $\psi(x)$ are strongly (asymptotically) equivalent if

$$
\varphi(x)=\psi(x)\left[1+o\left(|x|^{\omega \varepsilon}|\psi(x)|^{\omega_{2} \varepsilon}\right)\right]
$$

for some $\varepsilon<0$, where

$$
\omega_{2}= \begin{cases}-1 & \text { if } \psi(x) \rightarrow 0 \\ 0 & \text { if } \psi(x) \rightarrow \text { const } \neq 0 \\ 1 & \text { if } \psi(x) \rightarrow \infty\end{cases}
$$

In this case the function $\varphi(x)$ is a strong asymptotics of the function $\psi(x)$ and vice versa. 
Let $y=\varphi(x)$ be a solution of equation (1.1.4) and let $\varphi(x)$ have order $r$. The normal cone $\mathbf{u}$ of this solution is the ray

$$
\mathbf{u}= \begin{cases}\lambda(0,-1) & \text { if } r=-\infty \\ \lambda \omega(1, r) & \text { if }-\infty<r<+\infty \\ \lambda(0,1) & \text { if } r=+\infty\end{cases}
$$

where $\lambda>0$. The notion of the cone of a solution can be used for solutions with the normal cone $\mathbf{u}$ defined in such a way.

Theorem 1.3.1 ([3, Chpater VI, Theorem 1.1]). If a solution $y=\varphi(x)$ of equation (1.1.4) has a normal cone $\mathbf{u}$, then the reduced equation (1.1.9) for which $\mathbf{u} \subset \mathbf{U}_{j}^{(d)}$ has a weakly asymptotically equivalent solution $y=\psi(x)$.

Therefore, to compute all (weak) asymptotics of solutions of equation (1.1.4) one needs to identify all reduced equations and their normal cones, which leads to a finite number of problems of the following type.

Problem 3.2. For a reduced equation (1.1.9) find all (weak) asymptotics of solutions $y=\psi(x)$ of the form (1.3.1) with $\mathbf{u} \in \mathbf{U}_{j}^{(d)}$.

According to 1.1 , to equation (1.1.4) there correspond the polygon $\Gamma(f)$, and to the reduced equation (1.1.9), an edge or a vertex $\Gamma_{j}^{(d)}$ of this polygon. Below we consider separately three methods of treating Problem 3.2. two for an edge (depending on the slope of the edge) and one for a vertex.

Remark 1.3.1. The algebraic reduced equation (1.1.9) does not have appropriate nonpower solutions; i.e., it does not produce nonpower asymptotics of solutions of (1.1.4).

3.2. The case of a vertical edge $\Gamma_{j}^{(1)}$. If the edge $\Gamma_{j}^{(1)}$ is vertical, then its normal cone is

$$
\mathbf{U}_{j}^{(1)}=\lambda \omega(1,0), \quad \lambda>0,
$$

and all points $Q=\left(q_{1}, q_{2}\right) \in \Gamma_{j}^{(1)}$ have the same coordinate $q_{1}$. Let

$$
g(x, y)=x^{-q_{1}} \hat{f}_{j}^{(d)}(x, y) .
$$

Then the support $\mathbf{S}(g)$ lies on the coordinate axis $q_{1}=0$. According to (1.3.2) all power solutions (1.1.7) with $\omega(1, r) \in \mathbf{U}_{j}^{(1)}$ are constants $y=y^{0}=$ const, where $y^{0}$ is a root of the equation

$$
\tilde{g}(y) \stackrel{\text { def }}{=} g(0, y)=0 .
$$

A root $y^{0}$ of (1.3.4) is called a multiple root if the derivative $\frac{d \tilde{g}(y)}{d y}$ vanishes at $y^{0}$.

To find nonpower solutions of equation (1.1.9) we make the logarithmic transform

$$
\xi \stackrel{\text { def }}{=} \ln x \text {. }
$$

According to Theorem 2.4 in [3, Chapter VI], (1.3.5) transforms the differential sum $g(x, y)$ into the differential sum $h(\xi, y) \stackrel{\text { def }}{=} g(x, y)$ and equation (1.1.9) takes the form

$$
h(\xi, y)=0 .
$$

From (1.3.5) we see that $\xi \rightarrow \infty$ as $x \rightarrow 0$ and as $x \rightarrow \infty$ because both $\xi$ and $x$ are complex; i.e., for equation (1.3.6) we get Problem 3 with the cone

$$
p_{1} \geq 0 .
$$


Theorem 1.3.2 $([8, \S 5])$. Finite limit values $y^{0} \neq 0$ of nonconstant solutions of (1.3.6) are multiple solutions of equation (1.3.4).

Let an edge $\Gamma_{j}^{(1)}$ join two vertices $\Gamma_{j-1}^{(0)}=\left(q_{1}^{\prime}, q_{2}^{\prime}\right)$ and $\Gamma_{j}^{(0)}=\left(q_{1}^{\prime}, q_{2}^{\prime \prime}\right)$, where $q_{2}^{\prime}<q_{2}^{\prime \prime}$ are integers. We say that equation (1.3.4) has a root at infinity if the degree of the polynomial in (1.3.4) is less than $q_{2}^{\prime \prime}$, and that it has a root at zero if the smallest degree in $y$ of the polynomial in (1.3.4) is greater than $q_{2}^{\prime}$.

Theorem 1.3.3 ([8, $\S 5])$. If equation (1.3.4) does not have a root at infinity (at zero) then equation (1.3.6) does not have solutions that tend to infinity (to zero).

Therefore, if equation (1.3.4) does not have multiple roots, as well as roots at infinity and at zero, then equation (1.1.9) does not have appropriate nonpower solutions; i.e., we do not need to make the transformation (1.3.5). In the opposite case, we need to make the transformation (1.3.5) and to consider equation (1.3.6).

Let us note that the reduction of equation (1.3.6) with respect to the vector $(1,0)$ is equation (1.3.4), i.e., $\tilde{g}(y) \equiv \hat{h}(\xi, y)$. To find solutions of equation (1.3.6) with infinite and zero limit values of $y$ one should select in this equation the reduced equations corresponding to the cone of the problem (1.3.7). To find solutions of equation (1.3.6) with finite limit values, one should find all multiple roots of equation (1.3.4). Let $y^{0}$ be such a root. Then we need to make the parallel shift $y=y^{0}+z$ that will put this solution to the origin. Then equation (1.3.6) takes the form

$$
\tilde{h}(\xi, z) \stackrel{\text { def }}{=} h\left(\xi, y^{0}+z\right)=0 .
$$

Now one should consider all reduced equations of equation (1.3.8) with the cone $p_{1} \geq 0$, $p_{2} \leq 0$. This is again Problem 3.1

So, for a vertical edge we have described a step that allows us to find all power solutions of equation (1.1.9) and reducing, in the case of nonpower asymptotics, Problem 3.2 to a finite number of cases of Problem 3.1 .

An edge with nonzero slope can be transformed to a vertical edge using a power transform $[8, \S 5]$.

3.3. The case of a horizontal edge $\Gamma_{j}^{(1)}$. In this case all points $Q=\left(q_{1}, q_{2}\right)$ of the edge $\Gamma_{j}^{(1)}$ have the same coordinate $q_{2}$. Set

$$
g(x, y) \stackrel{\text { def }}{=} y^{-q_{2}} \hat{f}_{j}^{(1)}(x, y)
$$

and make the logarithmic transform

$$
\eta=\frac{d \ln y}{d x} .
$$

According to Theorem 2.4 in [3, Chapter VI] under this transform the differential sum $g(x, y)$ becomes the differential sum $h(x, \eta) \stackrel{\text { def }}{=} g(x, y)$ and equation (1.1.9) becomes

$$
h(x, \eta)=0 .
$$

Now for equation (1.3.11) we have Problem 3.1 with the cone

$$
p_{1}+p_{2} \geq 0 \text {. }
$$

The sum of orders of all derivatives entering a differential monomial $a(x, y)$ is called the total differential order of the monomial $a(x, y)$; it is denoted by $\Delta(a)$. For a differential sum (1.1.3) the total differential order is defined by

$$
\Delta(f)=\max _{i} \Delta\left(a_{i}\right)
$$


A. D. BRUNO AND I. V. GORYUCHKINA

Let $\Omega_{j}$ be the degree of the characteristic polynomial $\chi_{j}(r)$ of the vertex $\Gamma_{j}^{(0)}$. Let the edge $\Gamma_{j}^{(1)}$ join the vertices $\Gamma_{j-1}^{(0)}=\left(q_{1}^{\prime}, q_{2}^{\prime}\right)$ and $\Gamma_{j}^{(0)}=\left(q_{1}^{\prime \prime}, q_{2}^{\prime}\right)$ with $q_{1}^{\prime}<q_{1}^{\prime \prime}$.

Theorem 1.3.4 ([8, $\S 5])$. If $\Omega_{j-1}=\Delta\left(\hat{f}_{j-1}^{(0)}\right)=\Delta\left(\hat{f}_{j}^{(1)}\right) \quad\left(\right.$ or $\Omega_{j}=\Delta\left(\hat{f}_{j}^{(0)}\right)=$ $\Delta\left(\hat{f}_{j}^{(1)}\right)$ ), then equation (1.3.11) does not have appropriate solutions as $x \rightarrow 0$ (or as $x \rightarrow \infty)$.

Therefore, if $\Omega_{j-1}=\Delta\left(\hat{f}_{j-1}^{(0)}\right)=\Delta\left(\hat{f}_{j}^{(1)}\right)=\Delta\left(\hat{f}_{j}^{(0)}\right)=\Omega_{j}$, then transformation (1.3.10) and subsequent analysis can be omitted.

3.4. The case of a vertex $\Gamma_{j}^{(0)}$. Let $\Gamma_{j}^{(0)}=\{Q\}$. Set

$$
g(x, y) \stackrel{\text { def }}{=} X^{-Q} \hat{f}_{j}^{(0)}(x, y) \text {. }
$$

Then $\mathbf{S}(g)=\{0\}$. A power solution $y=c x^{r}$ of equation $g(x, y)=0$ contains an arbitrary constant $c$ and the exponent $r$ that satisfies the characteristic equation

$$
\chi(r) \stackrel{\text { def }}{=} g\left(x, x^{r}\right)=0 .
$$

To find nonpower solutions we make the logarithmic transform

$$
\xi=\ln x, \quad \eta=\frac{d \ln y}{d \ln x} .
$$

According to Theorem 2.4 in [3, Chapter VI] the differential sum $g(x, y)$ becomes the differential sum $h(\xi, \eta) \stackrel{\text { def }}{=} g(x, y)$ and equation (1.1.9) takes the form

$$
h(\xi, \eta)=0 .
$$

From (1.3.14) one sees that $\xi \rightarrow \infty$, i.e., $p_{1} \geq 0$. Therefore, for equation (1.3.15) we have Problem 3.1 with the cone $p_{1} \geq 0, p_{1}+p_{2} \geq 0$. Now we note that, according to (1.3.14), to a power solution (1.1.7) of equation (1.1.9) there corresponds the constant solution

$$
\eta=r=\mathrm{const}
$$

of equation (1.3.15).

Theorem 1.3.5 $([8, \S 5])$. Finite limit values $\eta^{0} \neq 0$ of nonconstant solutions of (1.3.15) are multiple roots of equation (1.3.13).

Let $\Delta(g(x, y))=k$. Denote by $g^{*}(x, y)$ the sum of all differential monomials in the sum $g(x, y)$ that have the total differential order $k$, i.e., the maximal order among all monomials in $g(x, y)$. By coef $\left(g^{*}\right)$ we denote the sum of all numeric coefficients in differential monomials in the sum $g^{*}(x, y)$.

Theorem 1.3.6 ([8, $\S 5])$. If the differential sum $g(x, y)$ in (1.3.12) contains a nonzero constant (or if coef $\left(g^{*}\right) \neq 0$ ), then equation (1.3.15) does not have solutions that tend to zero (or to infinity).

We say that a polynomial $\chi_{j}(r)$ does not have a root at infinity for a vertex $\Gamma_{j}^{(0)}$ if the fact that one if the vectors $\pm(0,1)$ belongs to $\mathbf{U}_{j}^{(0)}$ implies that $\Omega_{j}=\Delta\left(\hat{f}_{j}^{(0)}\right)$.

Corollary 1.3.1. If the characteristic polynomial $\chi_{j}(r)$ for the vertex $\Gamma_{j}^{(0)}$ does not have multiple roots $r$ such that at least one of the vectors $\pm(1, r)$ is in the normal cone $\mathbf{U}_{j}^{(0)}$ and does not have a root at infinity, then there are no nonpower asymptotics corresponding to the vertex $\Gamma_{j}^{(0)}$. 
Therefore, if the characteristic equation for $\hat{f}_{j}^{(0)}(x, y)$ does not have multiple roots, the sum $g(x, y)$ has a nonzero free term, and coef $\left(g^{*}\right) \neq 0$, then equation (1.1.9) does not have appropriate nonpower solutions.

Let us note that the reduction of equation (1.3.15) with respect to the vector $(1,0)$ is equation (1.3.13), i.e., $\chi(\eta) \equiv \hat{h}(\xi, \eta)$. To find solutions of equation (1.3.15) with infinite and zero limit values one should select those reduced equations for equation (1.3.15) for which the normal cone is not disjoint from the cone of the problem $p_{1} \geq 0, p_{1}+p_{2} \geq 0$, and then solve Problem 3.2 for these equations. To find solutions of equation (1.3.15) with finite nonzero limit values one should find all multiple roots of equation (1.3.13). Let $\eta^{0}$ be such a root. Using the parallel shift $\eta=\eta^{0}+\zeta$ one should put it at the origin. Equation (1.3.15) becomes

$$
\tilde{h}(\xi, \zeta) \stackrel{\text { def }}{=} h\left(\xi, \eta^{0}+\zeta\right)=0 .
$$

Now one should select reductions of equation (1.3.17) with the cone $p_{1} \geq 0, p_{2} \leq 0$. This is again Problem 3.1 .

\section{Complichted expansions}

4.1. Formulation of the problem. Earlier we have shown how to find nonpower solutions

$$
y=x^{r} \varphi_{r}(\ln x), \quad r \in \mathbb{R},
$$

of the reduced equation (1.1.9). These solutions can serve as nonpower asymptotics of solutions of the full equation (1.1.4). Here $\varphi_{r}$ is expanded in a series in powers of $\ln x$ :

$$
\varphi_{r}=\gamma_{\rho}(\ln x)^{\rho}+\sum \gamma_{\sigma}(\ln x)^{\sigma}, \quad \sigma<\rho,
$$

where $\gamma_{\rho}=$ const and the coefficients $\gamma_{\sigma}$ are either constants or polynomials in iterated logarithms (which does not occur in our case).

Problem 4.1. For nonpower asymptotics (1.4.1), (1.4.2) that is a solution of the reduced equation (1.1.9) and satisfies the condition $\omega(1, r) \in \mathbf{U}_{j}^{(d)}$, find the expansion of the corresponding solution of the full equation (1.1.4)

$$
y=\varphi_{r} x^{r}+\sum \varphi_{s} x^{s}, \quad \omega s<\omega r,
$$

where $\varphi_{s}$ are series in decreasing powers of logarithms.

Here we present a solution of this problem in the case where the reduced equation (1.1.9) corresponds to a vertex or to a nonhorizontal edge and satisfies certain conditions (does not produce critical numbers for the solution (1.4.1), (1.4.2)). In this case we will show how to obtain expansion (1.4.3) with coefficients $\varphi_{s}$ expanding in series in decreasing powers of the logarithm of the form

$$
\varphi_{s}=\sum \varphi_{s t}(\ln x)^{t}, \quad t \leq T(s),
$$

where the coefficients $\varphi_{\text {st }}$ are constant.

4.2. Computation of critical numbers. Consider the first variation (1.1.16). This is a linear differential operator $\mathcal{M}(x, y)$ whose coefficients are differential sums. Let us make the transformation

$$
y=x^{r} z,
$$

where $c$ is the same as in (1.4.1). We obtain the operator

$$
\mathcal{N}(x, z) \stackrel{\text { def }}{=} \mathcal{M}(x, y) \text {. }
$$


Now we make the logarithmic change of variables

$$
\xi=\ln x .
$$

Then

$$
\mathcal{N}(x, z) \stackrel{\text { def }}{=} x^{v} \tilde{\mathcal{N}}(\xi, z),
$$

where $v$ is the degree of the operator $\mathcal{N}$ in $x$ and $\tilde{\mathcal{N}}=\sum_{l=0}^{m} \pi_{l}(\xi, z)\left(\frac{d^{l}}{d \xi^{l}}\right)$ is a differential operator in $\xi$ whose coefficients are differential sums in $\xi$ and $z$. According to (1.4.2) let us substitute, in each coefficient $\pi_{l}, z=\varphi_{r}(\xi)$ and consider the terms with the highest degree of $\xi$. Let $n$ be this highest degree, i.e.,

$$
\pi_{l}\left(\xi, \varphi_{r}(\xi)\right)=\alpha_{l} \xi^{n}+\cdots, \quad \alpha_{l}=\mathrm{const}, \quad l=0,1, \ldots, m, \quad \sum_{l=0}^{m}\left|\alpha_{l}\right| \neq 0 .
$$

Let us set

$$
\tilde{\mathcal{N}}_{n}=\xi^{n} \sum_{l=0}^{m} \alpha_{l} \frac{d^{l}}{d \xi^{l}}
$$

Then $\tilde{\mathcal{N}}=\tilde{\mathcal{N}}_{n}+\cdots$. The polynomial

$$
\nu(k) \stackrel{\text { def }}{=} \sum_{l=0}^{m} \alpha_{l} k^{l}
$$

is called the characteristic polynomial for the reduced solution (1.4.1), (1.4.2). Its roots $k_{1}, \ldots, k_{m}$ are the eigenvalues of the solution (1.4.1), (1.4.2). Those eigenvalues that lie in the cone of the problem, i.e., satisfy $\omega k_{i}<\omega r$, are the critical numbers of the reduced solution (1.4.1), (1.4.2).

4.3. Computation of the support of an expansion. Let us recall that if a power asymptotics (1.1.7) with $r \in \mathbb{R}$ does not have critical numbers, then the exponents $s$ in the expansion (1.1.6) run over a set $\mathbf{K} \subset \mathbb{R}$. For a nonpower asymptotics (1.4.1), by $\mathbf{K}$ we will understand the same set as in the case of the power asymptotics.

Theorem 1.4.1 (9). If a reduced solution (1.4.1), (1.4.2) does not have critical numbers, then to this solution there corresponds a unique expansion (1.4.3), (1.4.4). In this expansion, the exponents $s$ run over the set $\mathbf{K}$ and (1.4.3), (1.4.4) do not contain iterated logarithms provided there are no iterated logarithms in (1.4.1), (1.4.2).

Theorem 1.4.2 ([9]). If under the hypotheses of Theorem 1.4 .1 expansion (1.4.2) does not contain iterated logarithms, then in expansions (1.4.3), (1.4.4) we have $T(s) \leq$ $s(\rho q-n)$, where $\rho$ is from (1.4.2) $q=\max q_{2}$ for points $\left(q_{1}, q_{2}\right)$ in the support $\mathbf{S}(f)$, and $n$ is from (1.4.9).

Hence, in the case of a power asymptotics the coefficients $\beta_{s}$ in the corresponding expansion (1.1.6) of a solution of the original equation (1.1.4) are at most polynomials in $\ln x$. In the case of a nonpower asymptotics of the form (1.4.1), (1.4.2) the coefficients $\beta_{s}$ in the corresponding expansion (1.1.6) are power series in decreasing powers of $\ln x$ and the exponents are unbounded from below.

Remark 1.4.1. As a rule, finding the entire expansion (1.4.2) requires an infinite number of steps, and the same is true for expansion (1.4.4). However, one can always compute initial terms of expansion (1.4.2) and several initial expansions (1.4.4) in a finite number of steps. 


\section{EXOTIC EXPANSIONS}

5.1. Series with purely imaginary exponents. We assume that the complex variable $x$ runs over the universal cover, i.e., $x=\exp (\rho+i \varphi), \rho+i \varphi=\operatorname{Ln} x$, where $i^{2}=-1$, $\rho, \varphi \in \mathbb{R}, \rho=\ln |x|$. Let $\alpha=\beta+i \gamma$ be a complex number, where $\beta, \gamma \in \mathbb{R}$ and $\gamma \neq 0$. Then the power function is given by

$$
z^{\alpha}=\exp [(\rho+i \varphi)(\beta+i \gamma)]=\exp [\rho \beta-\varphi \gamma+i(\rho \gamma+\varphi \beta)]
$$

and

$$
\left|x^{\alpha}\right|=\exp (\rho \beta-\varphi \gamma)
$$

The real linear function $\varphi=a \rho+b$, where $a, b=$ const $\in \mathbb{R}$, determines a line on the universal cover. On this line,

$$
\left|x^{\alpha}\right|=\exp [\rho(\beta-\gamma a)-b \gamma]
$$

and as $\rho \rightarrow-\infty$ we have

$$
\lim [\rho(\beta-\gamma a)-b \gamma]= \begin{cases}-\infty & \text { if } \beta-\gamma a>0 \\ -b \gamma & \text { if } \beta-\gamma a=0 \\ +\infty & \text { if } \beta-\gamma a<0\end{cases}
$$

Therefore,

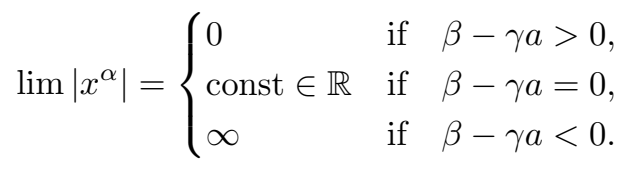

We have $\lim |x|=0$ and $\lim \left|x^{\alpha}\right|=\infty$ if $\beta<\gamma a$, i.e., $\operatorname{sgn} a=\operatorname{sgn} \beta \gamma$ and $|a|>\frac{|\beta|}{|\gamma|}$. This means that for each $\gamma \neq 0$ and $\beta$ there is a path $\varphi=a \rho+b$ on the universal cover such that $|x| \rightarrow 0$ and $\left|x^{\alpha}\right| \rightarrow \infty$. In particular for $\beta=0$ the equality $\operatorname{sgn} a=\operatorname{sgn} \gamma$ suffices.

Therefore, the power function $x^{\alpha}$ with a complex exponent $\alpha$ has a rather complicated behavior if $\varphi=\arg x$ is unbounded both from above and from below. Therefore in this paper we will assume that $\varphi$ is bounded from at least one side.

Now we consider the series

$$
\eta(x)=\sum_{k=0}^{\infty} c_{k} x^{i \gamma k},
$$

where $c_{k}=$ const $\in \mathbb{C}$ and $\gamma=$ const $\in \mathbb{R}$. If $\gamma>0$, then, according to (1.5.1),

$$
\left|x^{i \gamma k}\right|=\exp (-\varphi \gamma k)=[\exp (-\varphi \gamma)]^{k} .
$$

Therefore, for $\varphi \gamma>0$ the series (1.5.2) converges absolutely together with the corresponding power series. By the Cauchy formula, this happens whenever

$$
\exp (-\varphi \gamma)<\frac{1}{\overline{\lim _{k \rightarrow \infty}} \sqrt[k]{\left|c_{k}\right|} \stackrel{\text { def }}{=} \delta}
$$

If $\delta>0$ and $\gamma>0$, then inequality (1.5.3) holds for $-\varphi \gamma<\ln \delta$, i.e., for

$$
\varphi>-\gamma^{-1} \ln \delta \text {. }
$$

Let us note that the convergence region of the series (1.5.2) and $x^{A} \eta(x)$ is the same for all $A=$ const $\in \mathbb{C}$ provided we have excluded the points $x=0$ and $x=\infty$. Therefore, in all the examples below these points are assumed to be excluded. 


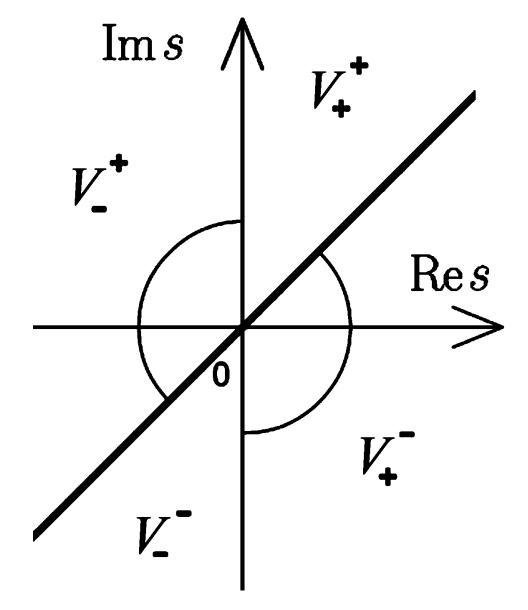

Figure 3. Vertical angles $V_{\sigma}^{\tau}$.

Consider the following generalization of the series (1.5.2):

$$
\zeta(x)=\sum_{k=0}^{\infty} c_{k} x^{s_{k}}
$$

where $c_{k}, s_{k}=$ const $\in \mathbb{C}$, $\operatorname{Re} s_{k}=0,\left|\operatorname{Im} s_{k+1}\right|>\left|\operatorname{Im} s_{k}\right| \geq 0, s_{k}$ do not have accumulation points and all $\operatorname{Im} s_{k}$ have the same sign. For the region of absolute convergence of this series we have

$$
\varphi \operatorname{sgn}\left(\operatorname{Im} s_{2}\right)>\operatorname{sgn}\left(\operatorname{Im} s_{2}\right) \varlimsup_{k \rightarrow \infty} \frac{\ln \left|c_{k}\right|}{\left|s_{k}\right|} .
$$

For the one-sided series (1.5.5) with all $\operatorname{Im} s_{k}$ of the same sign, the convergence region is either $\varphi>\varphi_{0}$ if $\operatorname{Im} s_{k} \geq 0$, or $\varphi<\varphi_{0}$ if $\operatorname{Im} s_{k} \leq 0$. We say that the series (1.5.5) belongs to the class $\mathcal{P}^{+}$if $\operatorname{Im} s_{k} \geq 0$ and to the class $\mathcal{P}^{-}$if $\operatorname{Im} s_{k} \leq 0$. Similarly, the series $x^{A} \zeta(x)$ is said to belong to subclass $\mathcal{P}^{+}$or $\mathcal{P}^{-}$depending on the class of the series $\zeta(x)$ in (1.5.5).

5.2. Exotic series. Suppose we are given the following two lines on the complex plane with coordinates $\operatorname{Re} s, \operatorname{Im} s$, both passing through the origin $s=0$ : the imaginary axes Im $s$ and a different from it inclined line (see Figure 3). These two lines divide the plane into four sectors $V_{\sigma}^{\tau}, \sigma, \tau= \pm 1$ (see Figure 3). We will assume that each sector is closed, i.e., contains its boundary. Here the subscript $\sigma$ in the notation of the angle $V_{\sigma}^{\tau}$ indicates the sign of $\operatorname{Re} s$ on the inclined part of the boundary, whereas the superscript $\tau$ indicates the sign of $\operatorname{Im} s$ on the vertical part of the boundary. The power series

$$
\xi(x)=\sum c_{s} x^{s} \quad(\text { sum over } s \in \mathbf{K}),
$$

$c_{s}=$ const $\in \mathbb{C}$, is said to belong to the class $\mathcal{P}_{\sigma}^{\tau}$ if for its support we have $\mathbf{K} \subset V_{\sigma}^{\tau}$. Furthermore, any power series of the form $x^{A} \xi(x)$ is assumed to belong to the same class. We will consider only those series of the form (1.5.7) for which the support $\mathbf{K}$ does not have limit points in the complex plane $s$. The series (1.5.7) of the class $\mathcal{P}_{\sigma}^{\tau}$ are called exotic. According to 5.1 the convergence region on the universal cover $\mathcal{P}_{\sigma}^{\tau}$ can be of the form

$$
\sigma \rho<\sigma \rho_{0}, \quad \tau \varphi<\tau \varphi_{0},
$$




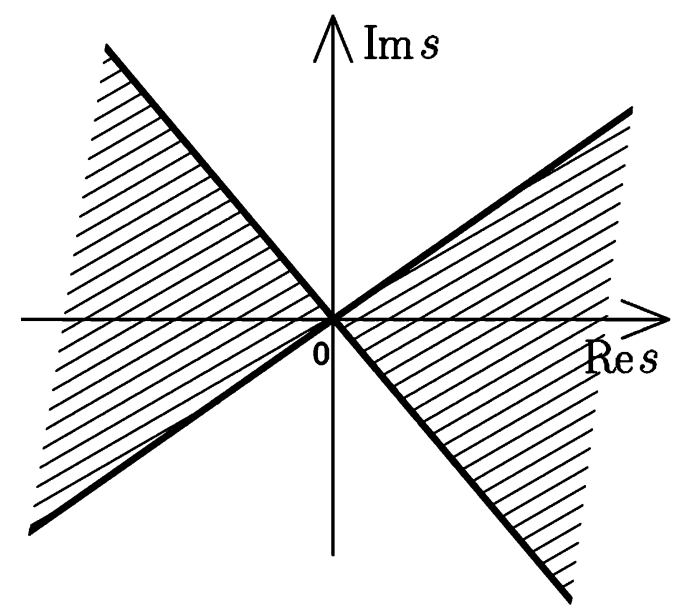

FiguRE 4. Horizontal sectors.

where $\rho_{0}$ and $\varphi_{0}$ are some real constants. The series (1.5.7) of class $\mathcal{P}_{\sigma}^{\tau}$ is asymptotic as $|x|^{\sigma} \rightarrow 0$ and $\varphi \rightarrow \tau \infty$ because in this case $\left|x^{\alpha}\right| \geq\left|x^{\beta}\right|$ for $\sigma \operatorname{Re} \alpha \leq \sigma \operatorname{Re} \beta, \tau \operatorname{Im} \alpha \leq$ $\tau \operatorname{Im} \beta$, which yields an ordering of monomials $x^{\alpha}$ by the exponents $\alpha$.

5.3. Existence of exotic expansions of solutions. Let us have an ordinary differential equation

$$
f(x, y)=0,
$$

where $f$ is a differential sum, and $y=y(x)$ is a solution of this equation. We are looking for exotic expansions of $y$ as $|x| \rightarrow 0$ and $|x| \rightarrow \infty$,

$$
y=c_{r} x^{r}+\sum c_{s} x^{s} \quad(\text { sum over } s \in \mathbf{K}, s \neq r),
$$

where the support $\mathbf{K}$ lies in the vertical sector $r+V_{\sigma}^{\tau}$ with the vertex at the point $r$. In the case of an ordinary expansion (1.5.10), where the set $\mathbf{K}-r$ lies in one "horizontal angle" between two inclined half-lines (see Figure 4), a procedure for finding them was described in Chapter 1, §2 Such expansions belong to both classes $\mathcal{P}_{\sigma}^{ \pm}$simultaneously.

If an exotic expansion (1.5.10) belongs to class $\mathcal{P}_{\sigma}^{\tau}$, then $\sigma=-\omega$.

Let us write an exotic expansion of a solution of (1.5.9) in the form

$$
y=\sum_{\rho} c_{\rho} x^{\rho}+\sum_{s} c_{s} x^{s}
$$

where $\operatorname{Re} \rho=r, \omega r>\omega \operatorname{Re} s$ and the first sum contains more than one summand.

Lemma 1.5.1. If expansion (1.5.11) is a formal expansion of a solution of the full equation (1.5.9) and $\omega(1, r) \in \mathbf{U}_{j}^{(d)}$, then the reduced solution

$$
y=\sum_{\rho} c_{\rho} x^{\rho}
$$

is a solution of the reduced equation

$$
\hat{f}_{j}^{(d)}(X)=0 .
$$

According to $\$ 1.2$, to equation (1.5.9) there corresponds the polygon $\Gamma(f)$ with the boundary $\partial \Gamma$ consisting of generalized faces $\Gamma_{j}^{(d)}$, and to each such face there corresponds 


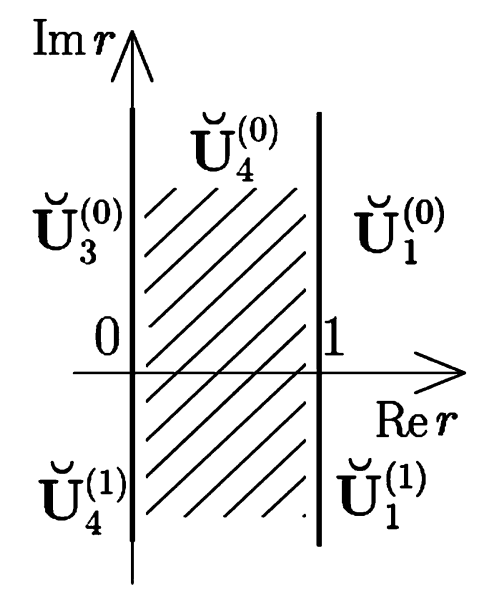

Figure 5. Complex normal cones.

a (real) normal cone $\mathbf{U}_{j}^{(d)}$; to the side face there also corresponds a particular value $\omega=\omega_{j}^{(d)}$.

By the complex normal cone $\breve{\mathbf{U}}_{j}^{(d)}$ of a face $\Gamma_{j}^{(d)}$ we mean the set of points $\rho \in \mathbb{C}$, where $\omega_{j}^{(d)}(1, \operatorname{Re} \rho) \in \mathbf{U}_{j}^{(d)}$. Complex normal cones $\breve{\mathbf{U}}_{j}^{(d)}$ can be represented in two complex planes $L_{\omega}$ depending on the sign $\omega=\omega_{j}^{(d)}= \pm 1$. For an edge $\Gamma_{j}^{(1)}$, the complex normal cone $\breve{\mathbf{U}}_{j}^{(1)}$ is the line $\operatorname{Re} \rho=r_{j}$; for a vertex, it is the strip $r_{j}<\operatorname{Re} \rho<r_{j+1}$ (shaded in Figure 5).

When looking for power-logarithmic expansions of the form (1.5.10) we assumed that $\omega \operatorname{Re} r>\omega \operatorname{Re} s$; i.e., after substituting $y=c_{r} x^{r}+z$ for $z$ we took the cone of the problem $\mathcal{K}=\{s: \omega \operatorname{Re} s<\omega \operatorname{Re} r\}$. Therefore in formula (1.2.5) we have only those $t_{i}$ and $k_{1}-r$ for which $\omega \operatorname{Re} t_{i}<0$ and $\omega \operatorname{Re}\left(k_{1}-r\right)<0$. Now in formula 1.2.5) we allow for the presence of purely imaginary $t_{i}$ and $k_{1}-r$. In this case the set $\mathbf{K}\left(k_{1}\right)$ may contain infinitely many points $k$ (or $s$ ) with fixed $\operatorname{Re} k$ (or Res) and the expansion with such support is exotic.

Let $\Gamma_{j}^{(1)}$ be an edge of the polygon $\Gamma(f)$ adjacent to a vertex $\Gamma_{k}^{(0)}$ and let $N_{j}=\left(1, r_{j}\right)$ be the real normal to this edge. Then the vector $M_{j}=\left(-r_{j}, 1\right)$ is directed along the edge $\Gamma_{j}^{(1)}$ and $t_{j}=r-r_{j}$. This last number is purely imaginary if $\operatorname{Re} r=r_{j}$ and $\operatorname{Im} r \neq 0$. This means that the complex number $r$ lies in the complex normal cone $\breve{\mathbf{U}}_{j}^{(1)}$ of the edge $\Gamma_{j}^{(1)}$. Furthermore, the complex number $r$ is a root of the characteristic equation $\chi_{k}(r)=0$ of the vertex $\Gamma_{k}^{(0)}$. Therefore, there are exotic expansions corresponding to a vertex $\Gamma_{j}^{(1)}$ if one of the adjacent vertices $\Gamma_{k}^{(0)}$ has a root $r$ of the characteristic equation belonging to the complex normal cone $\breve{\mathbf{U}}_{j}^{(1)}$ of the edge $\Gamma_{j}^{(1)}$. The purely imaginary difference $k_{1}-r$ occurs in the cases where the power asymptotics $y=c_{r} x^{r}$ has an eigennumber $k_{1}$ such that $\operatorname{Re} k_{1}=\operatorname{Re} r$ and $k_{1} \neq r$. Therefore, for the extended expansion we have two cones of the problem:

$$
\mathcal{K}^{\tau}=\{s: \text { either } \omega \operatorname{Re} s<\omega \operatorname{Re} r \text { or } \operatorname{Re} s=\operatorname{Re} r \text { and } \operatorname{sgn} \operatorname{Re}(s-r)=\tau\} .
$$

For each side edge $\Gamma_{j}^{(1)}$ there are two adjacent vertices $\Gamma_{k}^{(0)}$ and $\Gamma_{k+1}^{(0)}$, one being the top vertex, and another, the bottom vertex for this edge. 
Hence, to each face $\Gamma_{j}^{(d)}$ of the polygon $\Gamma(f)$ there corresponds a complex normal cone $\breve{\mathbf{U}}_{j}^{(d)}$. With this notation, Theorem 1.1 .2 can be generalized as follows.

Theorem 1.5.1. Exotic expansions of solutions (1.5.10) of equation (1.5.9) correspond only to side edges of the polygon $\Gamma(f)$, and this happens in the following two cases (here $\left(1, r_{j}\right)$ is the normal vector to the edge $\left.\Gamma_{j}^{(1)}\right)$ :

1. For a vertex $Q_{k}^{(0)}$ adjacent to the side edge $\Gamma_{j}^{(1)}$ the characteristic equation $\chi_{k}(\rho)$ $=0$ has a root $\rho=r_{j}+i \gamma$ with $\gamma \neq 0$.

2. The defining equation $\tilde{f}_{j}(c)=0$ corresponding to the edge $\Gamma_{j}^{(1)}$ has a root $c_{0}$ with the eigenvalue of the form $\lambda=r_{j}+i \gamma, \gamma \neq 0$.

Here $\tau=\operatorname{sgn} \gamma$ in Case 2 and for the bottom vertex in Case 1 , and $\tau=-\operatorname{sgn} \gamma$ for the top vertex in Case 1.

Exotic solutions corresponding to a vertex $\Gamma_{k}^{(0)}=Q_{k}^{(0)}$ exist only in the following case:

3. The reduced solution $y=c_{r} x^{r}$ has a critical number $k_{1}$ with $\operatorname{Re} k_{1}=\operatorname{Re} r$ and $k_{1} \neq r$.

For the equation P6, Case 3 does not happen and all exotic solutions correspond to edges.

In all cases in Theorem 1.5.1 the value of $\tau$ is determined and the cone of the problem $\mathcal{K}$ is unique. Therefore, critical numbers are determined uniquely. In other cases there are two cones $\mathcal{K}^{\tau}$ and each cone has its own set of critical numbers. Critical numbers $k_{j}$ with $\operatorname{Re} k_{j}>\omega \operatorname{Re} r$ are common for both $\mathcal{K}^{+}$and $\mathcal{K}^{-}$, whereas critical points with Re $k_{j}=\operatorname{Re} r$ are different for different $\tau$.

With these definitions all constructions and results in $\S 2$ remain valid when $k_{i}$ is a complex critical number.

Now Theorem 1.2.1 about solutions of equation (1.5.9) allows us to obtain exotic expansions (1.5.10), where the $c_{s}$ are polynomials in $\ln x$. The exponents $s$ in expansion (1.5.10) can be ordered as follows. According to Propositions 1.2 .2 and 1.2 .3 the set $\mathbf{K}\left(k_{1}\right)-r$ lies in some central sector $V$ with vertex at the origin such that either at least one of the sides of the angle lies on an inclined line, or one of the sides lies on the axis $\operatorname{Im} s$, i.e., the angle $V$ is of the form $V_{\sigma}^{\tau}$. Let the vectors $P_{1}$ and $P_{2}$ be exterior normals to the sides of the angle $V$. Denote $P=P_{1}+P_{2}=\left(p_{1}, p_{2}\right)$. Then for each point $s=s^{\prime}+i s^{\prime \prime} \in \mathbb{C}$, set $\|s\|=p_{1} s^{\prime}+p_{2} s^{\prime \prime}$. We say that a point $s \in \mathbb{C}$ precedes a point $t \in \mathbb{C}$ if $\|s\|>\|t\|$. Expansion (1.5.10), i.e., the coefficients $c_{s}$ for $s \in \mathbf{K}$ are computed consecutively following this ordered sequence of exponents $s$ as was described in Lemmas 1.2 .1 and 1.2 .2 .

Let us note that the support and the coefficients of an exotic expansion are computed by the same formulas as for power-logarithmic expansions.

For the complex normal cones shown in Figure 5 the position of the angles $V_{\sigma}^{\tau}$ is shown in Figure 6 depending on $\operatorname{Re} r$ : a) for $\operatorname{Re} r=0$, b) for $0<\operatorname{Re} r<1$, c) for $\operatorname{Re} r=1$.

Remark 1.5.1. In the cases described in Theorem 1.5.1, solution (1.5.10) has an infinite series of the form

$$
y=\sum_{k=1}^{\infty} c_{k} x^{r+k i \gamma},
$$

which is a solution of the reduced equation

$$
\hat{f}_{j}^{(1)}(x, y)=0,
$$




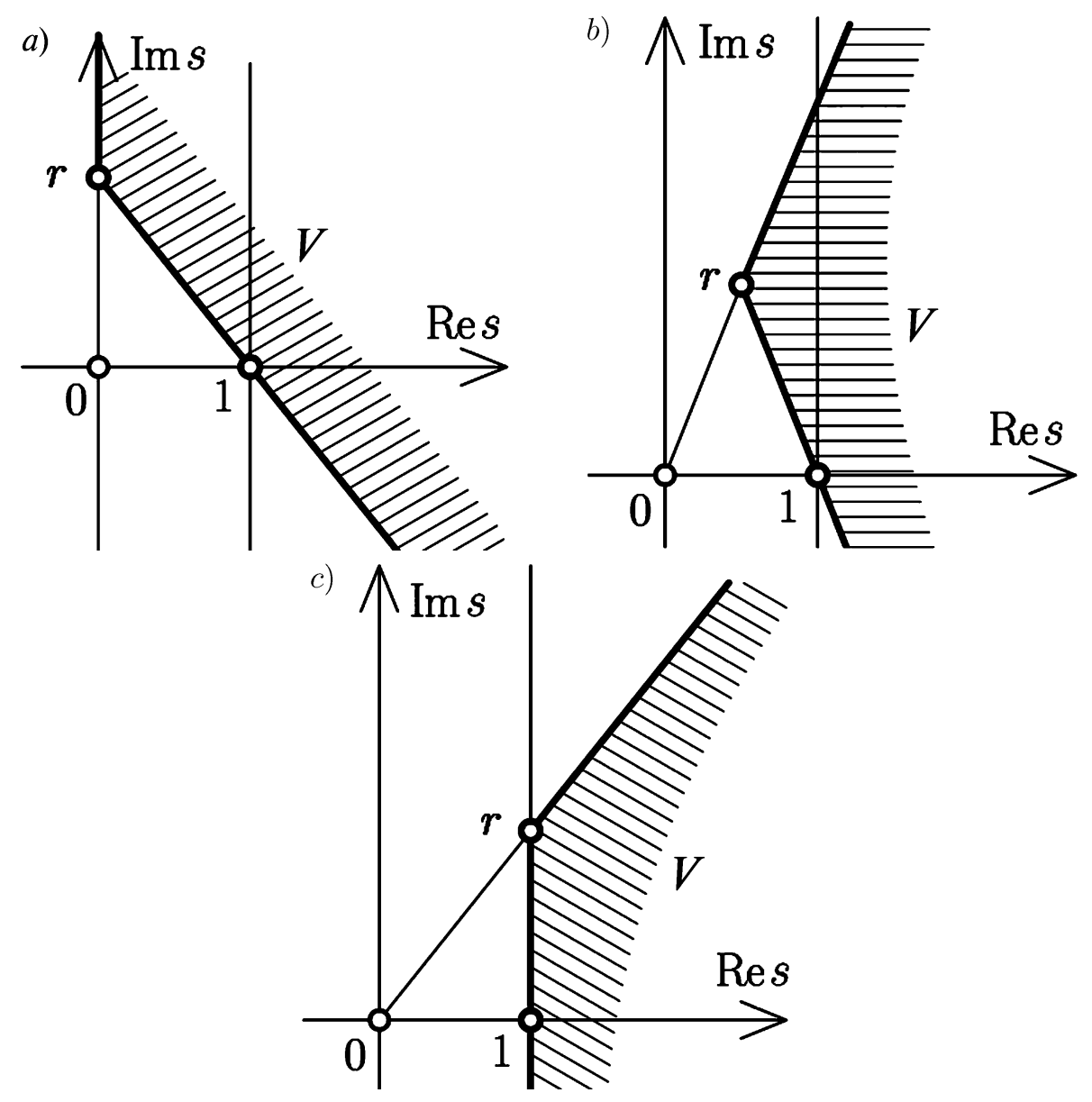

Figure 6. The sector $V$ of expansion (2.2.6) for $(a) \operatorname{Re} r=0 ;(b)$ $\operatorname{Re} r \in(0,1) ;(c) \operatorname{Re} r=1$.

corresponding to the edge $\Gamma_{j}^{(1)}$. However, it may happen that this series consists of just one term

$$
y=c_{1} x^{r+i \gamma} .
$$

In this case (1.5.15) is a root of each summand $f_{Q}(x, y)$ in the differential sum

$$
\hat{f}_{j}^{(1)}(x, y)=\sum f_{Q} \quad\left(\text { sum over } Q \in \mathbf{S}\left(\hat{f}_{j}^{(1)}\right)\right) .
$$

The complex support of the differential sum $\mathcal{L}(x) z$ consists of several points, which correspond to points $Q$ of the support $\mathbf{S}\left(\hat{f}_{j}^{(1)}\right)$. The two extremes of these points correspond to the vertices of the edge $\Gamma_{j}^{(1)}$. To each of these two vertices there corresponds its own support: $\mathbf{K}_{\mathrm{T}}$ to the top vertex and $\mathbf{K}_{\mathrm{B}}$ to the bottom vertex. However, in this case the support of the expansion (1.5.10) is $\mathbf{K}=\mathbf{K}_{\mathrm{T}} \cap \mathbf{K}_{\mathrm{B}}$, so it is power-logarithmic rather than exotic.

5.4. Semiexotic expansions. An expansion (1.5.11) for which the first sum contains a finite number (but more than one) of summands is called semiexotic. Let $\min (\operatorname{Im} \rho)$ in (1.5.11) be achieved at $\rho=\rho_{*}$, and $\max (\operatorname{Im} \rho)$ at $\rho=\rho^{*}$. Let $\mathbf{K}_{*}$ be the support of 
an exotic expansion containing the point $\rho_{*}$, and $\mathbf{K}^{*}$ the support of the exotic expansion with the point $\rho^{*}$.

Theorem 1.5.2. The support of a semiexotic expansion (1.5.11) is $\mathbf{K}_{*} \cap \mathbf{K}^{*}$.

Consider a Laurent polynomial with real exponents

$$
y(x)=\sum_{k=m}^{n} c_{k} x^{k},
$$

where $c_{k} \in \mathbb{C}$ and $m \neq n$. The inverse function $y^{-1}$ has two different power expansions: near zero, $x=0$,

$$
y_{0}^{-1}=c_{m}^{-1} x^{-m}\left(1+\sum_{k=1}^{\infty} b_{k} x^{k}\right)
$$

and near infinity, $x=\infty$,

$$
y_{\infty}^{-1}=c_{n}^{-1} x^{-n}\left(1+\sum_{k=1}^{\infty} \tilde{b}_{k} x^{-k}\right)
$$

where $b_{k}$ and $\tilde{b}_{k} \in \mathbb{C}$.

Similarly, we have the following result.

Theorem 1.5.3. The semiexotic expansion (1.5.11) has two inverse exotic expansions

$$
\left(y^{-1}\right)_{*}=c_{\rho_{*}}^{-1} x^{-\rho_{*}}\left(\sum_{\sigma} b_{\sigma} x^{\sigma}+\sum_{t} b_{t} x^{t}\right)
$$

and

$$
\left(y^{-1}\right)^{*}=c_{\rho^{*}}^{-1} x^{-\rho^{*}}\left(\sum_{\sigma} \tilde{b}_{\sigma} x^{\sigma}+\sum_{t} \tilde{b}_{t} x^{t}\right)
$$

where $\operatorname{Re} \sigma=0$.

According to Theorem 1.5.2 the semiexotic expansion (1.5.11) belongs to both classes $\mathcal{P}^{+}$and $\mathcal{P}^{-}$, and each of two exotic expansions (1.5.19) and (1.5.20) belongs to just one of the classes $\mathcal{P}^{+}$or $\mathcal{P}^{-}$, and the signs of their classes are opposite to one another.

Expansion (1.5.11) for which $\operatorname{Im} \rho$ is not bounded from above is called superexotic. However, Painlevé equations do not have superexotic expansions.

\section{OTHER TYPES OF ASYMPTOTICS AND EXPANSIONS}

6.1. Formulation of the problem. Two-dimensional power geometry [8, $\S 5]$ allows us to find nonpower (exponential) asymptotics of solutions

$$
y \sim \exp \left(c_{r} x^{r}+\sum_{s} c_{s} x^{s}\right),
$$

corresponding to a horizontal edge. However, equation P6 does not have asymptotics of this type.

Similarly, power geometry [ $8, \S 7]$ allows us to find exponential small additional terms to power and power-logarithmic expansions. However, equation P6 does not have additional terms of this type either. 


\section{NONFORMAL SOLUTIONS}

At present, we have the following general results about the existence of an actual solution with the previously found formal solution as its asymptotic expansion.

Theorem 1.7.1 ([38]). If a system

$$
\frac{d X}{d t}=\frac{F(X)}{g(X)},
$$

where $F(X)$ and $g(X)$ are real and analytic near zero ( $F$ is a vector), $F(0)=0, g(0)=0$, has a real formal solution $\stackrel{\circ}{X}(t)$, with the expansion containing only real powers of $t$, iterated logarithms of $t$, or exponents, and if all coefficients are real, then this system has an actual solution $X(t)$. The formal expansion $\stackrel{\circ}{X}(t)$ is an asymptotic expansion for $X(t)$, i.e.,

$$
\left|X(t)-\sum_{k=0}^{m} \Phi_{k}(t)\right|=o\left(\Phi_{m}(t)\right), \quad m=1,2, \ldots,
$$

where $\stackrel{\circ}{X}(t)=\sum_{k=0}^{\infty} \Phi_{k}(t)$. Here $X, F$, and $\Phi$ are $n$-dimensional vectors.

Equation (2) can be reduced to the system (1.7.1) by setting

$$
\dot{x}=1, \quad \dot{y}=z, \quad \dot{z}=h(x, y, z),
$$

where $h$ is the right-hand side of (2) and $z=y^{\prime}$, provided that we consider real values of $a, b, c, d$ only. In this case Theorem 1.7 .1 shows that there exist actual solutions corresponding to real formal solutions. It is reasonable to expect that Theorem 1.7.1 can be generalized to complex formal solutions if the latter are considered on a line through the origin $t=0$ in the complex plane $t$.

In the case where all parameters $a, b, c, d$ of the sixth Painlevé equation are real, Theorem 1.7.1 can be applied to the following families of basic formal solutions of types 1-3 (with real arbitrary constants) listed in the Introduction: the family $\mathcal{A}_{0}$ with real $r \in(0,1)$; the families $\mathcal{B}_{i}$ with $\operatorname{Re} \theta_{i} \neq 0$ and $c_{k_{i} i}=0$ in the case $\operatorname{Im} \theta_{i} \neq 0, i=1,2$; the family $\mathcal{B}_{3}$; the families $\mathcal{B}_{4}, \mathcal{B}_{5}$ and $\mathcal{B}_{6}$ with $a>0$; the families $\mathcal{C}_{0}^{\infty}, \mathcal{B}_{8}, \mathcal{B}_{9}$ with $c>0$; the family $\mathcal{B}_{10}$.

In the case of equation (1.2.2), if expansion (1.2.6) converges for sufficiently small $|x|^{-\omega}$, then there is a solution of (1.2.2) corresponding to this equation. The maximal order of derivatives in a differential sum $f(x, z)$ is called the differential order and is denoted by $\pi(f)$.

Theorem 1.7.2 ([8, Theorem 3.4]). The power expansion (1.2.1) of a solution of equation (1.2.2), where $\tilde{f}$ is a differential sum, converges for sufficiently small $|x|^{-\omega}$ and $\left|\arg x-\mu_{0}\right|$, where $\mu_{0}=$ const $\in[0,2 \pi]$, provided that

$$
\pi(\mathcal{L}(x) z)=\pi(\tilde{f}) .
$$

Here we prove this theorem in the case where the power expansion (1.2.1) contains only integral powers. The case of rational exponents with the finite common denominator $m$ can be reduced to this one using a substitution $\tilde{x}=x^{1 / m}$. The restriction on $\arg x$ is used only in the case of expansions (1.2.1) with irrational or complex exponents, and in our case it can be omitted.

Proof. We prove the theorem in a neighborhood of the point $x=0$, so that $\omega=-1$. Let equation (1.2.2) have a formal solution in the form of a power series

$$
y=\sum_{k=k_{0}}^{\infty} c_{k} x^{k}
$$


with constant coefficients $c_{k} \in \mathbb{C}$. In equation (1.2.2) make the change of variables

$$
y=\sum_{k=k_{0}}^{k_{m}} c_{k} x^{k}+u,
$$

where $k_{m} \geq k_{0}$. As a result, the equation takes the form

$$
f_{1}(x, u) \stackrel{\text { def }}{=} \mathcal{L}(x) u+g\left(x, u, u^{\prime}, \ldots, u^{(n)}\right)=0
$$

where $\mathcal{L}(x)$ is a linear differential operator. The function $f_{1}$ contains integral powers of $x$, and $u, u^{\prime}, \ldots, u^{(n)}$ contain integral nonnegative powers of $x$. Equation (1.7.5) can also be multiplied by the minimal power of $x$. Therefore, we will assume that $f_{1}$ is a polynomial in the variables $x, u, u^{\prime}, \ldots, u^{(n)}$. Equation (1.7.5) has the following properties:

1. $\mathbf{S}(\mathcal{L}(x) u)=(v, 1)$

2. $(v, 1)$ is a vertex of the polygon $\Gamma\left(f_{1}\right)$;

3. $\mathbf{S}\left(f_{1}\right) \subset\left\{q_{2} \geq 0\right\}$

4. $\mathbf{S}(g) \cap\left\{q_{2}=1\right\} \subset\left\{q_{1}>v\right\}$

5. $\mathbf{S}(g) \cap\left\{q_{2}=0\right\} \subset\left\{q_{1} \geq v+k_{m}+1\right\}$.

Equation (1.7.5) has a formal solution

$$
u(x)=\sum_{k=k_{m}+1}^{\infty} c_{k} x^{k} .
$$

The linear differential operator $\mathcal{L}(x)$ has the eigenvalues $\lambda_{1}, \ldots, \lambda_{n}$, which can be ordered by the increase of the real part $\operatorname{Re} \lambda_{1} \leq \operatorname{Re} \lambda_{2} \leq \cdots \leq \operatorname{Re} \lambda_{n}$.

For the vertex $(v, 1)$ of the polygon $\Gamma\left(f_{1}\right)$, let $\Gamma_{1}^{(1)}$ be the upper adjacent edge and $-(1, r)$ the corresponding outward normal vector (see Figure (7) $(a)$ ). Denote by $[r]$ the integral part of $r$ if $r>0$ and 0 if $r \leq 0$.

In (1.7.4), let

$$
k_{m}: \quad k_{m}>\operatorname{Re} \lambda_{n} \quad \text { and } \quad k_{m}>[r]+1+2 n .
$$

The expansion (1.7.7) is unique, where all $c_{k}$ are constant and uniquely determined. The edge $\Gamma_{1}^{(1)}$ and the corresponding reduced equation do not depend on $k_{m}$ (see Figure $7(b)$ ).

If the support $\mathbf{S}\left(f_{1}\right)$ of equation (1.7.5) has points to the left of the line $q_{1}=v$, we apply to equation (1.7.5) the following power transformation:

$$
u=x^{\alpha} w \quad \text { with } \quad \alpha=[r]+1+n .
$$

As a result, we obtain an equation with the support lying in the set $\left\{q_{1} \geq v, q_{2} \geq 0\right\}$ and satisfying properties (1.7.6), where on the right-hand side in Property 5 we have $\left\{q_{1} \geq v+n+1\right\}$. The transformed polygon $\tilde{\Gamma}\left(f_{1}\right)$ is drawn by dashed lines in Figure $7(b)$. We assume that equation (1.7.5) itself is such that the support $\mathbf{S}\left(f_{1}\right)$ belongs to the set $\left\{q_{1} \geq v, q_{2} \geq 0\right\}$ and Property 5 in (1.7.6) with $n$ instead of $k_{m}$ holds.

Next we use majorization and also a certain new trick. Consider the series

$$
\varphi(x)=\sum_{k=k_{m}+1}^{\infty} C_{k} x^{k}
$$

where $C_{k} \in \mathbb{R}, C_{k} \geq 0$. The series (1.7.10) majorizes the series (1.7.7), in the case where

$$
C_{k} \geq\left|c_{k}\right|, \quad k=k_{m}+1, \ldots
$$

Let us construct the equation

$$
\sigma \varphi^{(n)}=x G\left(x, \varphi^{(n)}\right), \quad \sigma=\text { const } \in \mathbb{R}, \quad \sigma>0,
$$



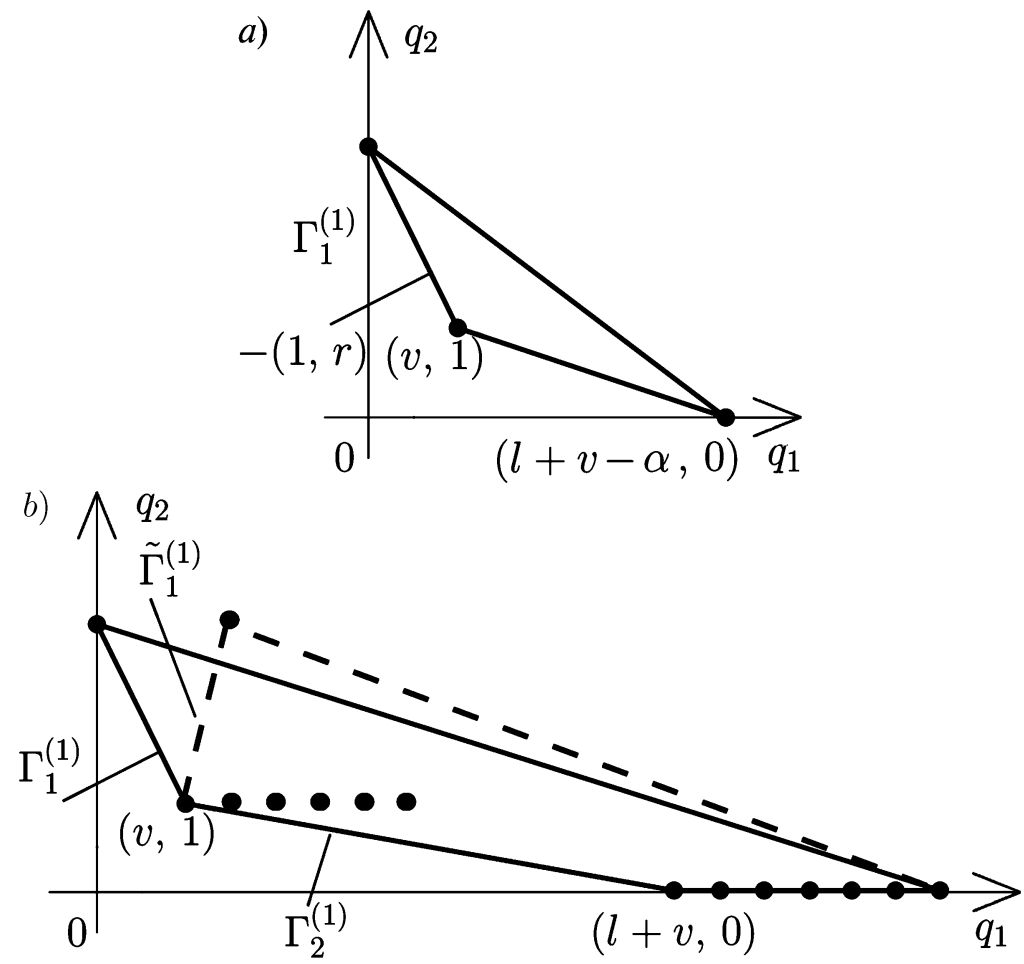

Figure 7. (a) The left part of the polygon of equation (1.2.2); (b) the support and the polygon of equation (1.7.5) before (solid line) and after (dashed line) transformation (1.7.9).

with expansion (1.7.10) serving the role of a formal solution and also majorizing the solution (1.7.7) of equation (1.7.5). To do this, we write equation (1.7.5) in the form

$$
\mathcal{L}(x) u=-g\left(x, u, u^{\prime}, \ldots, u^{(n)}\right) .
$$

In the characteristic polynomial $\nu(k)$ of the differential sum $\mathcal{L}(x) u$, let the coefficient $\nu_{n}$ in the term of highest power $k^{n}$ be different from 1. By condition (1.7.2) we have $\nu_{n} \neq 0$. Dividing the equation by $\nu_{n}$, we obtain an equation for which $\nu_{n}=1$. We will assume that this holds already for the initial equation (1.7.5). Then for $k>n$ we have

$$
|\nu(k)| \geq \sigma k(k-1) \cdots(k-n+1),
$$

where $\sigma$ is a positive number. Since $\mathcal{L}(x) x^{k}=x^{k+v} \nu(k)$, for $x>0$ and $k>n$ we have

$$
\left|\mathcal{L}(x) x^{k}\right| \geq \sigma x^{v+n} \frac{d^{n}}{d x^{n}} x^{k} .
$$

Using the function $-g$ we construct a function $G^{*}\left(x, u^{(n)}\right)$ as follows. Replace all coefficients $\beta_{i}$ of the polynomial $-g\left(x, u, u^{\prime}, \ldots, u^{(n)}\right)$ with their absolute values $\left|\beta_{i}\right|$. Next, replace the function $u$ and all its derivatives $u^{(k)}$ with expressions $x^{n-k} u^{(n)}$. Let us note that if $u=x^{r}$, then $u^{(k)}=r(r-1) \cdots(r-k+1) x^{r-k}$, and $x^{n-k} u^{(n)}=$ $r(r-1) \cdots(r-n+1) x^{r-k}$, i.e., in the second case the coefficient is not smaller than in the first case. Therefore, the support of $\mathbf{S}(-g)$ coincides with the support of $\mathbf{S}\left(G^{*}\right)$. Since for the support $\mathbf{S}(g)$ we have $q_{1} \geq v+1$ the ratio $\frac{G^{*}\left(x, u^{(n)}\right)}{x^{v+n+1}} \stackrel{\text { def }}{=} G\left(x, u^{(n)}\right)$ is a polynomial in $x$ and $u^{(n)}$. Indeed, according to the above property, all the monomials $u^{(k)} x^{l}$ 
entering the polynomial $g$ satisfy the condition $l-k \geq v+1$. After replacements are made, such a monomial becomes the monomial $u^{(n)} x^{n-k} x^{l}$ with $n-k+l \geq v+1+n$. For monomials of the second degree in $u$ the situation is even simpler: the polynomial $g$ contains only monomials $u^{(j)} u^{(k)} x^{l}$ with $l-k-j \geq v+1$, and after the replacements this monomial becomes the monomial $\left(u^{(n)}\right)^{2} x^{n-k} x^{n-j} x^{l}$ with $2 n-k-j+l \geq v+1+2 n$. For monomials of higher degree in $u$, inequalities are even stronger. For monomials that do not contain $u$ the exponents can be made arbitrarily large by increasing the length of the initial part of the expansion of solution (1.7.3). For terms that are nonlinear in $u$ the degree in $x$ can be made arbitrarily large using a power transform. The terms that are linear in $u$ are the only ones that cannot be improved using these methods. However, these terms already possess the necessary property: $G^{*}\left(x, u^{(n)}\right) / x^{v+n+1}$ is a polynomial in $x$ and $u^{(n)}$. Hence, we have constructed equation (1.7.12) starting with equation (1.7.5).

Now we prove that solution (1.7.10) of equation (1.7.12) majorizes solution (1.7.7) of equation (1.7.5). The proof is by induction in $k$. Let us assume that inequalities (1.7.11) hold for $k<j$ and prove that they hold for $k=j$.

First we note that according to Subsection 2.3 in Chapter 1, when substituting the series (1.7.7) in equation (1.7.13), for each coefficient $c_{k}$ we obtain an equation of the following form:

$$
\mathcal{L}(x) c_{k} x^{k}=-b_{k} x^{k+v}, \quad k \geq k_{m}+1,
$$

where the coefficients $b_{k}$ depend on the coefficients $c_{k}$ with $k<j$ and on the coefficient of equation (1.7.13). According to Corollary 1.2.1, the system (1.7.15) can be rewritten in the form

$$
\nu(k) c_{k}=-b_{k}, \quad k \geq k_{m}+1 .
$$

In a similar way, for equation (1.7.12) we obtain the equalities

$$
\sigma k(k-1) \cdots(k-n+1) C_{k}=B_{k}, \quad k \geq k_{m}+1,
$$

where the coefficients $B_{k}$ are found using equation (1.7.12) in the same way as the coefficients $b_{k}$ are found using equation (1.7.5). Here $B_{j}$ is a polynomial with positive coefficients in $C_{k}$ and $B_{k}$ with $k<j$. Using the induction hypotheses and the above construction we see that $B_{j} \geq\left|b_{j}\right|$. Furthermore,

$$
\left|x^{-(v+j)} \mathcal{L}(x) x^{j}\right|=|\nu(j)| \geq \sigma j(j-1) \cdots(j-n+1) .
$$

Therefore

$$
C_{j}=\frac{B_{j}}{\sigma j(j-1) \cdots(j-n+1)} \geq\left|c_{j}\right|=\frac{\left|b_{j}\right|}{|\nu(j)|} .
$$

The statement for the start of the induction is obvious. Therefore, solution (1.7.10) of equation (1.7.12) majorizes solution (1.7.7) of equation (1.7.5).

Now we recall the analytic version of the Cauchy implicit function theorem; see 34]. Let a function $f(x, z)$ be analytic at zero, $x=z=0$ (i.e., $f$ can be expanded in a power series in $x$ and $z), f(0,0)=0$, and $\frac{\partial f}{\partial z} \neq 0$ for $x=z=0$. Then equation $f(x, z)=0$ has a unique solution

$$
z=\sum_{k=1}^{\infty} d_{k} x^{k}
$$

that is analytic near the point $x=0$; i.e., the series converges for sufficiently small $|x|$.

We apply this Cauchy theorem to equation (1.7.12) with $x=x, z=\varphi^{(n)}$, and $f(x, z)=\sigma \varphi^{(n)}-x G\left(x, \varphi^{(n)}\right)=\sigma z-x G(x, z)$. Here the function $f(x, z)$ is a polynomial; hence it is analytic at the point $x=z=0$. Furthermore, $f(0,0)=0$ and 
$\partial f / \partial z=\sigma \neq 0$ at the point $x=z=0$. Therefore, all the hypotheses of the Cauchy theorem are satisfied. According to this theorem, equation (1.7.12) has a unique solution

$$
z=\varphi^{(n)}=\sum_{k=k_{m}-n+1}^{\infty} d_{k} x^{k},
$$

which is analytic; i.e., the series converges for sufficiently small $|x|$.

Solution (1.7.10) is obtained by integrating solution (1.7.18) $n$ times; therefore, it is also analytic for small $|x|$. Finally, it majorizes solution (1.7.7) of equation (1.7.5). Therefore, solution (1.7.7) is analytic as well.

It is clear that $\pi\left(f_{1}\right)=\pi(f)$. However, in general, $\pi(\mathcal{L} u) \leq \pi(\hat{f})$, although a strong inequality occurs only in degenerate cases. For other conditions for the convergence of the formal solution (1.7.3) of (1.2.2), see [4, 44].

Theorem 1.7 .2 can be applied to the following families of basic formal solutions of Type 1 of the sixth Painlevé equation listed in the Introduction: the family $\mathcal{A}_{0}$; the families $\mathcal{B}_{i}$ with $\theta_{i} \notin \mathbb{Z}, i=1,2$; the families $\mathcal{B}_{6}, \mathcal{C}_{0}^{\infty}, \mathcal{B}_{10}$.

Currently, we do not have a general theory of convergence of exotic expansions (of Type 4 or 5). We only know some cases where such expansions sum to a particular function. For the P6 equation such cases were listed in the Introduction when we described basic families of formal solutions of the $\mathrm{P} 6$ equation.

\section{Chapter 2. Expansions of SOlutions of P6 NEAR Zero AND INFinity IN THE CASE $a \cdot b \neq 0$}

\section{General properties of the equation}

1.1. Formulation of the problem. The sixth Painlevé equation 39] has the form

$$
\begin{aligned}
y^{\prime \prime}= & \frac{\left(y^{\prime}\right)^{2}}{2}\left(\frac{1}{y}+\frac{1}{y-1}+\frac{1}{y-x}\right)-y^{\prime}\left(\frac{1}{x}+\frac{1}{x-1}+\frac{1}{y-x}\right) \\
& +\frac{y(y-1)(y-x)}{x^{2}(x-1)^{2}}\left[a+b \frac{x}{y^{2}}+c \frac{x-1}{(y-1)^{2}}+d \frac{x(x-1)}{(y-x)^{2}}\right],
\end{aligned}
$$

where $a, b, c, d$ are complex parameters, $x$ and $y$ are complex variables, $y^{\prime}=\frac{d y}{d x}$. This equation has three singular points $x=0, x=\infty$ and $x=1$.

For all values of the parameters of the equation, we are looking for asymptotics expansions of solutions of this equation that have the following form as $x \rightarrow 0$ or $x \rightarrow \infty$ :

$$
y=c_{r} x^{r}+\sum_{s} c_{s} x^{s}
$$

where the exponents $r$ and $s$ are complex numbers. According to (1.1.5), $\omega=-1$ if $x \rightarrow 0$ and $\omega=1$ if $x \rightarrow \infty$. In expansions (2.1.2) we have $\omega \operatorname{Re} s<\omega \operatorname{Re} r$ and $\omega \operatorname{Re} s$ decreases. We distinguish three types of expansions (2.1.2) depending on the form of the complex coefficients $c_{r}$ and $c_{s}$ :

1. $c_{r}$ and $c_{s}$ are constant coefficients (power expansion).

2. $c_{r}$ is a constant coefficient and $c_{s}$ are polynomials in $\ln x$ (power-logarithmic expansions).

3. $c_{r}$ and $c_{s}$ are power series in decreasing powers of $\ln x$ (complicated expansions).

Furthermore, we look for those asymptotic expansions of solutions of equation (2.1.1) that for $x \rightarrow 0$ or for $x \rightarrow \infty$ have the form

$$
y=\sum_{r} c_{r} x^{r}+\sum_{s} c_{s} x^{s}
$$



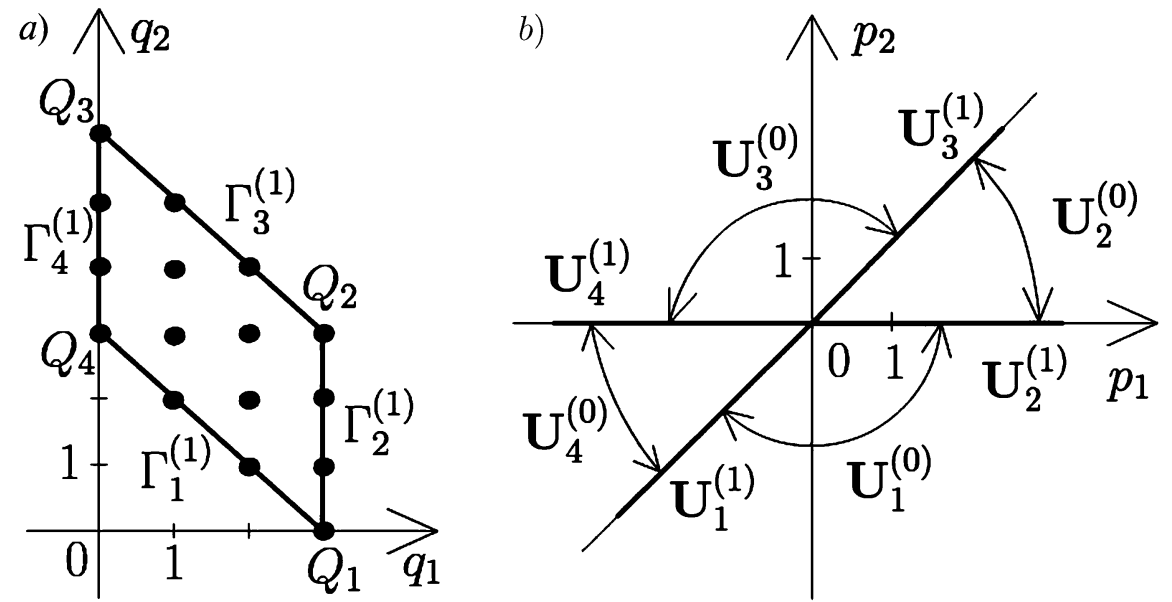

Figure 8. (a) The support of equation (2.1.4), its polygon, edges, and vertices; (b) normal cones of edges and vertices.

where all $\operatorname{Re} r$ are equal and $\omega \operatorname{Re} s<\omega \operatorname{Re} r$, the first sum contains more than one summand, and the coefficients $c_{r}$ and $c_{s}$ are polynomials in $\ln x$. We distinguish three types of expansions (2.1.3) depending on the structure of the first sum:

4. The first sum in (2.1.3) contains a finite number of terms and for the extreme values of $\operatorname{Im} r$ the coefficients $c_{r}$ are constants (semiexotic expansions).

5. The first sum in (2.1.3) contains infinitely many terms but $\operatorname{Im} r$ is bounded from one side. For the extreme value of $\operatorname{Im} r$ the coefficient $c_{r}$ is constant (exotic expansions).

6. The first sum in (2.1.3) contains an infinite number of terms and $\operatorname{Im} r$ is unbounded either from below or from above (superexotic expansions).

We also assume that $\arg x$ is bounded from both sides.

Remark 2.1.1. Since the support of equation (2.1.1) is real and power asymptotics $c_{r} x^{r}$ of solutions of the equation have at most one critical value, all coefficients in exotic expansions (2.1.3) of solutions of equation (2.1.1) are constant.

Let us represent equation (2.1.1) as a differential sum. To do this, we multiply it by $2 x^{2}(x-1)^{2} y(y-1)(y-x)$ and move the right-hand side of the equation to the left. We obtain the equation

$$
\begin{aligned}
f(x, y) \stackrel{\text { def }}{=} & 2 y^{\prime \prime} x^{2}(x-1)^{2} y(y-1)(y-x) \\
- & \left(y^{\prime}\right)^{2}\left[x^{2}(x-1)^{2}(y-1)(y-x)+x^{2}(x-1)^{2} y(y-x)+x^{2}(x-1)^{2} y(y-1)\right] \\
+ & 2 y^{\prime}\left[x(x-1)^{2} y(y-1)(y-x)+x^{2}(x-1) y(y-1)(y-x)+x^{2}(x-1)^{2} y(y-1)\right] \\
- & {\left[2 a y^{2}(y-1)^{2}(y-x)^{2}+2 b x(y-1)^{2}(y-x)^{2}\right.} \\
& \left.+2 c(x-1) y^{2}(y-x)^{2}+2 d x(x-1) y^{2}(y-1)^{2}\right]=0 .
\end{aligned}
$$

1.2. The support and the polygon. The support of the left-hand side of equation (2.1.4), i.e., the set of exponents of all entering monomials, is

$$
\mathbf{S}(f)=\left\{Q=\left(q_{1}, q_{2}\right): q_{1}=0,1,2,3, q_{2}=3-q_{1}+k, k=0,1,2,3\right\} .
$$

The support $\mathbf{S}(f)$ and its convex hull $\Gamma(f)$ are shown in Figure $8(a)$. 

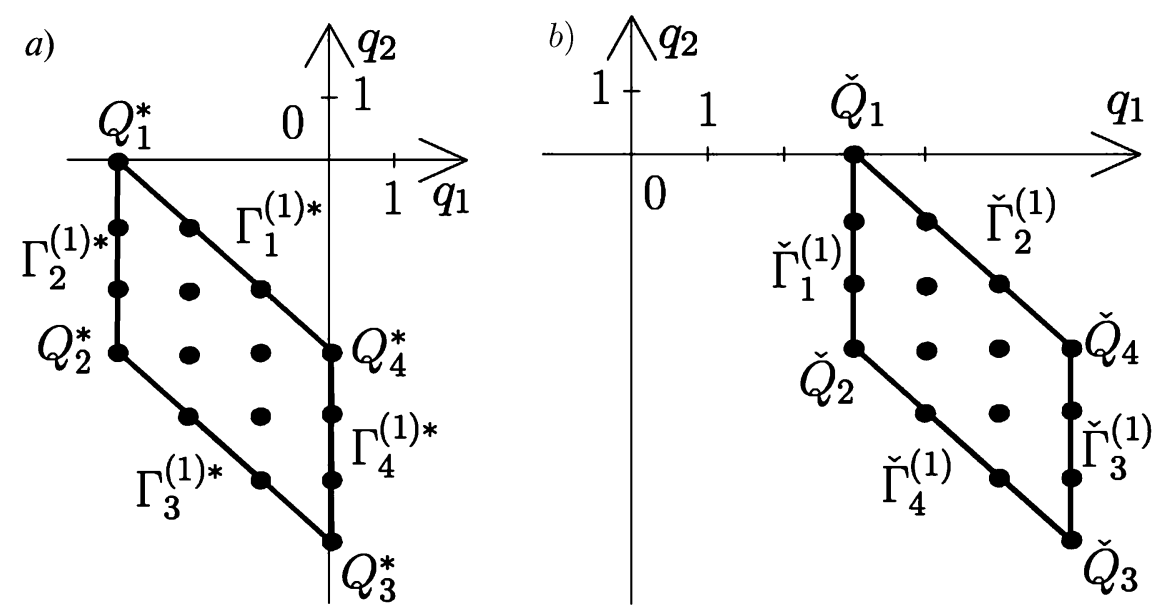

Figure 9. The polygon of equation (2.1.4) (a) after the substitution (2.1.6) and (b) after the substitution (2.1.8).

Since $a, b \neq 0$, the polygon $\Gamma(f)$ is the parallelogram with vertices $\Gamma_{j}^{(0)}=Q_{j}$, where $Q_{1}=(3,0), Q_{2}=(3,3), Q_{3}=(0,6), Q_{4}=(0,3)$, and edges $\Gamma_{1}^{(1)}, \Gamma_{2}^{(1)}, \Gamma_{3}^{(1)}, \Gamma_{4}^{(1)}$, shown in Figure $8(a)$. The support $\mathbf{S}(f)$ of equation (2.1.4) is a subset of the integral lattice $\mathbb{Z}^{2}$.

1.3. Normal cones. Real normal cones $\mathbf{U}_{j}^{(0)}$ and $\mathbf{U}_{j}^{(1)}$ of vertices $\Gamma_{j}^{(0)}$ and edges $\Gamma_{j}^{(1)}$ are (see Figure $8(b)$ )

$$
\begin{array}{ll}
\mathbf{U}_{1}^{(0)}=\left\{p_{2}<0, p_{2}<p_{1}\right\}, & \mathbf{U}_{1}^{(1)}=\left\{p_{1}=p_{2}<0\right\}, \\
\mathbf{U}_{2}^{(0)}=\left\{p_{1}>p_{2}>0\right\}, & \mathbf{U}_{2}^{(1)}=\left\{p_{1}>0, p_{2}=0\right\}, \\
\mathbf{U}_{3}^{(0)}=\left\{p_{2}>p_{1}, p_{2}>0\right\}, & \mathbf{U}_{3}^{(1)}=\left\{p_{1}=p_{2}>0\right\}, \\
\mathbf{U}_{4}^{(0)}=\left\{p_{1}<p_{2}<0\right\}, & \mathbf{U}_{4}^{(1)}=\left\{p_{1}<0, p_{2}=0\right\} .
\end{array}
$$

According to Subsection 5.3 in Chapter 1 , for $\omega=-1$ the decomposition of the complex plane $L_{-}: r \in \mathbb{C}$ into complex normal cones $\breve{\mathbf{U}}_{j}^{(d)}$ is as shown in Figure 5 ,

1.4. Symmetries. The sixth Painlevé equation has three main symmetries resulting from changes of variables:

$$
\text { 1) } x=\frac{1}{x^{*}}, y=\frac{1}{y^{*}} ; \quad \text { 2) } x=\check{x}, y=\frac{\check{x}}{\check{y}} ; \quad \text { 3) } x=1-x^{\circ}, y=1-y^{\circ} .
$$

Theorem 2.1.1. Equation (2.1.4) is invariant under the substitution

$$
(x, y, a, b, c, d)=\left(\frac{1}{x^{*}}, \frac{1}{y^{*}},-b^{*},-a^{*}, c^{*}, d^{*}\right) .
$$

Furthermore, under this substitution the parallelogram $\Gamma(f)$ reflects about the center $Q=$ $\left(\frac{3}{2}, 3\right)$ (see Figure $9(a)$ ).

Proof. Substituting $y=\frac{1}{y^{*}}$ we obtain $y^{\prime}=-\frac{y^{* \prime}}{y^{* 2}}, y^{* \prime \prime}=\frac{2 y^{* \prime}-y^{*} y^{* \prime \prime}}{y^{* 3}}$. Next, substituting $x=\frac{1}{x^{*}}$ we obtain $\frac{d x^{*}}{d x}=-x^{* 2}$ and $y^{* \prime}=-x^{* 2} \dot{y}^{*}, y^{* \prime \prime}=\ddot{y}^{*} x^{* 4}+2 \dot{y}^{*} y^{* 3}$, where the dot denotes the derivative in $x^{*}$. Therefore,

$$
y^{\prime}=x^{* 2} \dot{y}^{*} y^{*-2}, \quad y^{\prime \prime}=\frac{2 x^{* 4} \ddot{y}^{* 2}-x^{* 4} \ddot{y}^{*} y^{*}-2 x^{* 3} \dot{y}^{*} y^{*}}{y^{* 3}} .
$$


Substituting in (2.1.4) expressions (2.1.7), and setting $x=\frac{1}{x^{*}}$ and $y=\frac{1}{y^{*}}$ after cancellations, reduction of similar terms, and multiplication by $x^{* 3} y^{* 6}$ we obtain an equation of the form (2.1.4) where instead of the variables $x, y$ we have $x^{*}, y^{*}$, and instead of the parameters $a, b, c, d$ we have $-b^{*},-a^{*}, c^{*}, d^{*}$, respectively.

Theorem 2.1.2. Equation (2.1.4) does not change under the substitution

$$
(x, y, a, b, c, d)=(\check{x}, \check{x} / \check{y},-\check{b},-\check{a},-\check{d}+1 / 2,-\check{c}+1 / 2) .
$$

In addition, the parallelogram $\Gamma(f)$ reflects about the horizontal axis $q_{2}=3$ and is deformed parallel to this axis (see Figure $9(b)$ ).

Theorem 2.1.3. Equation (2.1.4) does not change under the substitution

$$
(x, y, a, b, c, d)=\left(1-x^{\circ}, 1-y^{\circ}, a^{\circ},-c^{\circ},-b^{\circ}, d^{\circ}\right) .
$$

Theorems 2.1.2 and 2.1.3 are proved similarly to Theorem 2.1.1

The first two symmetries send the expansion of a solution near zero to the expansion near infinity (and vice versa). Therefore, they allow us to reduce computations and to verify the obtained results. With these symmetries, any edge can be mapped to any other edge, and vertices $\Gamma_{3}^{(0)}=Q_{3}$ and $\Gamma_{4}^{(0)}=Q_{4}$ to vertices $\Gamma_{1}^{(0)}=Q_{1}$ and $\Gamma_{2}^{(0)}=Q_{2}$, respectively (and vice versa). Therefore, computing expansions of solutions corresponding to one edge and two vertices, we can use symmetries to obtain solutions corresponding to the other three edges and two vertices. The third symmetry sends expansions of solutions near zero to expansions of solutions near the point 1.

\subsection{Exceptional solutions.}

Theorem 2.1.4. Equation (2.1.1) has four exceptional solutions:

$$
\begin{array}{ll}
\mathcal{I}_{1}: & y(x)=0 \text { for } b=0 ; \\
\mathcal{I}_{2}: & y(x)=1 \text { for } c=0 ; \\
\mathcal{I}_{3}: & y(x)=x \text { for } d=\frac{1}{2} ; \\
\mathcal{I}_{4}: & y(x)=\infty \text { for } a=0 .
\end{array}
$$

Proof. We assume that for $y=$ const the square of the derivative $y^{\prime 2}$ is a zero of order 2. Therefore, the expressions $\frac{y^{\prime 2}}{y}$ for $y=0$ and $\frac{y^{\prime 2}}{y-1}$ for $y=1$ are simple zeros. Finally, for $y=x$, in the equation (2.1.1) with $d=\frac{1}{2}$ the fractions with the denominator $y-x$ mutually cancel. The symmetry (2.1.6) sends the case $y(x)=0$ for $b=0$ to the case $y(x)=\infty$ for $a=0$.

\section{EXPANSIONS NEAR ZERO CORRESPONDING TO VERTICES}

2.1. The choice of a vertex. Since $x \rightarrow 0$, we have $\omega=-1$ and the cone of the problem is given by $\mathcal{K}=\left\{p_{1}<0\right\}$. According to (2.1.5) the cone of the problem has a nonempty intersection with real normal cones $\mathbf{U}_{1}^{(0)}, \mathbf{U}_{3}^{(0)}, \mathbf{U}_{4}^{(0)}$ of vertices $\Gamma_{1}^{(0)}=Q_{1}$, $\Gamma_{3}^{(0)}=Q_{3}, \Gamma_{4}^{(0)}=Q_{4}$, respectively. Consider these real normal cones one by one.

Corresponding to the vertex $\Gamma_{1}^{(0)}$ is the reduced equation $\hat{f}_{1}^{(0)}(x, y) \stackrel{\text { def }}{=}-2 b x^{3}=0$, which has no solutions.

Corresponding to the vertex $\Gamma_{3}^{(0)}$ is the reduced equation $\hat{f}_{3}^{(0)}(x, y) \stackrel{\text { def }}{=}-2 a y^{6}=0$, which has only trivial solutions. 
2.2. Expansions of solutions corresponding to the vertex $\Gamma_{4}^{(0)}$. The vertex $\Gamma_{4}^{(0)} \stackrel{\text { def }}{=}$ $Q_{4}$ is the left bottom vertex; the corresponding value of $\omega$ is $\omega=-1$, and the corresponding reduced equation is

$$
\hat{f}_{4}^{(0)}(x, y) \stackrel{\text { def }}{=} 2 x^{2} y\left(y^{\prime}\right)^{2}-2 x y^{2} y^{\prime}-2 x^{2} y^{2} y^{\prime \prime}=0 .
$$

Corresponding to this reduced equation is the characteristic equation

$$
\chi(r) \stackrel{\text { def }}{=} 2\left(r^{2}-r-r^{2}+r\right) \equiv 0 ;
$$

it has an arbitrary solution $r \in \mathbb{C}$. Therefore, the solution of (2.2.1) is

$$
y=c_{r} x^{r}
$$

with arbitrary complex constants $c_{r}$ and $r$.

The real normal cone is given by $\mathbf{U}_{4}^{(0)}=\left\{p_{1}<p_{2}<0\right\}$. According to subsection 5.3 in Chapter 1, the complex normal cone is $\breve{\mathbf{U}}_{4}^{(0)}=-(1, r)$, where

$$
r: \quad r \in \mathbb{C}, \quad 0<\operatorname{Re} r<1 .
$$

Let us compute the critical numbers. The first variation is

$$
\frac{\delta \hat{f}_{4}^{(0)}(x, y)}{\delta y}=2 x^{2}\left(y^{\prime}\right)^{2}+4 x^{2} y y^{\prime} \frac{d}{d x}-4 x y y^{\prime}-2 x y^{2} \frac{d}{d x}-4 x^{2} y y^{\prime \prime}-2 x^{2} y^{2} \frac{d^{2}}{d x^{2}} .
$$

At the reduced solution $y=c_{r} x^{r}$ the first variation yields the following linear differential operator:

$$
\mathcal{L}(x) \stackrel{\text { def }}{=} 2 c_{r}^{2} x^{2 r}\left(r^{2}+2 r x \frac{d}{d x}-2 r-x \frac{d}{d x}-2 r(r-1)-x^{2} \frac{d^{2}}{d x^{2}}\right) .
$$

The characteristic equation

$$
\nu(k) \stackrel{\text { def }}{=} 2 c_{r}^{2}\left(k^{2}-2 r k+r^{2}\right)=0
$$

has a root $k_{1,2}=r$ of multiplicity 2 .

The cone of the problem is

$$
\mathcal{K}=\{\operatorname{Re} k>\operatorname{Re} r\} .
$$

Since the numbers $k_{1,2}$ are not in the cone of the problem, $\mathcal{K}$, they are not critical numbers. According to Subsection 2.2 in Chapter 1, the support of the expansion of solutions (2.1.2) is of the form

$$
\mathbf{K}=\{s=r+l r+m(1-r) ; l, m \geq 0 ; l+m>0 ; l, m \in \mathbb{Z}\} .
$$

To the solution (2.2.2) of the reduced equation (2.2.1) there correspond the following expansions of solutions of the full equation (2.1.4):

$$
\mathcal{A}_{0}: y=c_{r} x^{r}+\sum c_{s} x^{s} \quad(\text { sum over } s \in \mathbf{K}),
$$

where $r$ is given by (2.2.3), $\mathbf{K}$ by (2.2.5), and the complex coefficients are as follows: $c_{r}$ is a nonzero complex constant; all other $c_{s}$ are uniquely determined constants.

Let us study expansions (2.2.6). The support (2.2.5) has two generators, $r$ and $1-r$ and on the complex plane it lies inside the sector with the vertex at the point $r$ and with sides parallel to the vectors $(\operatorname{Re} r, \operatorname{Im} r)$ and $(\operatorname{Re}(1-r), \operatorname{Im}(1-r))$. For $\operatorname{Im} r>0$ the sector $V$ is shown in Figure 6(b). Expansions (2.2.6) are power expansions.

Therefore, we have proved the following theorem. 
Theorem 2.2.1. For $x \rightarrow 0$ there exists a two-parameter (with parameters $c_{r}$ and $r$ ) family of expansions (with constant coefficients) of solutions given by the formula

$$
\mathcal{A}_{0}: y=c_{r} x^{r}+\sum_{s} c_{s} x^{s}
$$

where $r$ is arbitrary with $\operatorname{Re} r \in(0,1), s \in \mathbf{K}=\{r+l r+m(1-r) ; l, m \geq 0 ; l+m>0$; $l, m \in \mathbb{Z}\}$, and the complex coefficients are as follows: $c_{r} \neq 0$ is an arbitrary complex constant; all other $c_{s}$ are uniquely determined complex constants.

The family $\mathcal{A}_{0}$ was known before. The convergence of this family for small $|x|$ was proved by different methods in 62, 64, 73, 74, 75, 76,. It follows also from Theorem 1.7 .2

For $\operatorname{Im} r=0$ the support (2.2.5) of expansion (2.2.6) is real.

Consider the case where $\operatorname{Re} r \neq 0, \operatorname{Re} r \neq 1$ and $\operatorname{Im} r \neq 0$. On the plane $\operatorname{Re} q_{1}, \operatorname{Im} q_{1}$ draw the set $\mathbf{K} \cup\{r\}$. Let, for example, $\operatorname{Im} r=1$. For different values of $\operatorname{Re} r, \operatorname{Re} r=\frac{1}{4}$, $\operatorname{Re} r=\frac{1}{2}, \operatorname{Re} r=\frac{3}{4}$, this set is shown in Figures 10 $(a)$, 10, $(b)$, 10 $(c)$ respectively. Figure 10.(b) shows that for $\operatorname{Re} r=\frac{1}{2}$ there are two values $\operatorname{Im} s=0$ and $\operatorname{Im} s=2$, corresponding to $\operatorname{Re} s=1$, which is different from the case $\operatorname{Im} r=0$ (see [12, 26]).

Let us compute the second approximation (2.2.6) in the case of a complex support (2.2.5).

In case $1>\operatorname{Re} r>\frac{1}{2}$ the second approximation to the solution is (see Figure 10 $(c)$ )

$$
y=c_{r} x^{r}+c_{1} x .
$$

The second approximation to the equation (2.1.4) is $\hat{\hat{f}}_{4}^{(0)}(x, y)=-x^{3}\left(y^{\prime}\right)^{2}+2 x^{3} y y^{\prime \prime}-$ $2(b-d) x y^{2}$. The coefficient $c_{1}$ equals $c_{1}=-\frac{b_{1}}{\nu(1)}$, where

$$
b_{1} \stackrel{\text { def }}{=} x^{-2 r-1} \hat{\hat{f}}_{4}^{(0)}\left(x, c_{r} x^{r}\right)=c_{r}^{2}\left(-2(b-d)-2 r+r^{2}\right),
$$

$\nu(1)=2 c_{r}^{2}(r-1)^{2}$. We obtain

$$
c_{1}=\frac{2(b-d)-(r-1)^{2}+1}{2(r-1)^{2}} .
$$

In case $0<\operatorname{Re} r<\frac{1}{2}$ the second approximation to the solution is (see 10 $(a)$ )

$$
y=c_{r} x^{r}+c_{2 r} x^{2 r} .
$$

The second approximation to the equation (2.1.4) is $\hat{\hat{f}}_{4}^{(0)}(x, y)=-3 x^{2} y^{2}\left(y^{\prime}\right)^{2}+2 x y^{3} y^{\prime}+$ $2 x^{2} y^{3} y^{\prime \prime}-2 a y^{4}+2 c y^{4}$. The coefficient $c_{2 r}$ is equal to $-b_{2 r} / \nu(2 r)$, where $b_{2 r} \stackrel{\text { def }}{=}$ $x^{-4 r} \hat{\hat{f}}_{4}^{(0)}\left(x, c_{r} x^{r}\right)=-c_{r}^{4}\left(2(a-c)+r^{2}\right), \nu(2 r)=2 c_{r}^{2} r^{2}$. We obtain

$$
c_{2 r}=c_{r}^{2} \frac{2(a-c)+r^{2}}{2 r^{2}} \text {. }
$$

In case $\operatorname{Re} r=\frac{1}{2}, \operatorname{Im} r \neq 0$ the second approximation to the solution is (see Figure [10(b)) $y=c_{r} x^{r}+c_{1} x+c_{2 r} x^{2 r}$, where the coefficients $c_{1}$ and $c_{2 r}$ are given by formulas (2.2.8) and (2.2.10) respectively.

In case $r=\frac{1}{2}$ the second approximation to the solution is (see Figure 10(b)) $y=$ $c_{\frac{1}{2}} \sqrt{x}+c_{1} x$, where $c_{\frac{1}{2}}$ is an arbitrary constant, and the coefficient

$$
c_{1}=\frac{3+8(b-d)+c_{\frac{1}{2}}^{2}+8 c_{\frac{1}{2}}^{2}(a-c)}{2}
$$

is the sum of (2.2.8) and (2.2.10). 


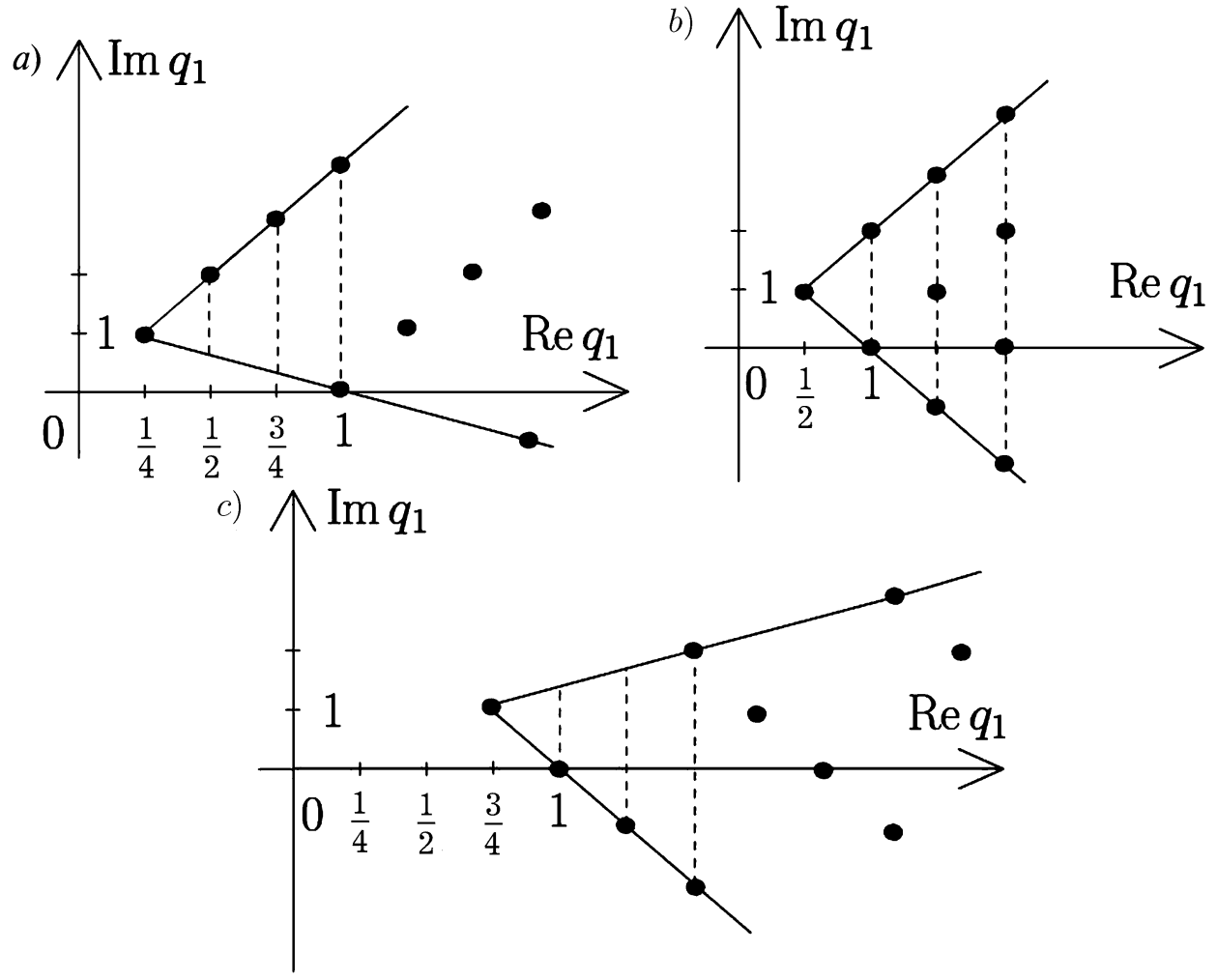

FiguRE 10. The support of expansion (2.2.6) for $(a) r=1 / 4+i ;(b)$ $r=\frac{1}{2}+i ;(c) r=3 / 4+i$.

Let us compute nonpower solutions of equation (2.2.1). Transform this equation as follows:

$$
g(x, y) \stackrel{\text { def }}{=} y^{-3} \hat{f}_{4}^{(0)}(x, y)=2 x^{2} \frac{\left(y^{\prime}\right)^{2}}{y^{2}}-2 x \frac{y^{\prime}}{y}-2 x^{2} \frac{y^{\prime \prime}}{y}=0,
$$

$\mathbf{S}(g)=\{0\}$. Set $g^{*} \stackrel{\text { def }}{=} 2 x^{2} \frac{\left(y^{\prime}\right)^{2}}{y^{2}}-2 x^{2} \frac{y^{\prime \prime}}{y}$. Then $\operatorname{coef}\left(g^{*}\right)=2-2=0$. According to Theorems 1.3.5 and 1.3.6 there may exist nonpower solutions of equation (2.2.1) that tend to infinity. In equation (2.2.12), make the logarithmic transform $\xi=\ln x, \eta=d \ln y / d \xi$. Denoting the derivative with respect to $\xi$ by the dot, after the substitution $\xi=\ln x$ we have

$$
y^{\prime}=\frac{\dot{y}}{x}, \quad y^{\prime \prime}=\frac{\ddot{y}-\dot{y}}{x^{2}} .
$$

Equation (2.2.12) takes the form

$$
2 \frac{\dot{y}^{2}-\ddot{y} y}{y^{2}}=0 .
$$

In the expression (2.2.14), make the transform $\eta=\frac{d \ln y}{d \xi}$. Then

$$
\dot{y}=\eta y, \quad \ddot{y}=\left(\dot{\eta}+\eta^{2}\right) y .
$$

We obtain the equation $-2 \dot{\eta}=0$. It has a solution $\eta=$ const, and the corresponding solution of the reduced equation (2.2.1) is the power solution $y=\tilde{c} x^{\eta}, \tilde{c}=$ const, i.e., 
(2.2.2); there are no other solutions of the reduced equation (2.2.1). Therefore, there are no complicated expansions of solutions of (2.1.4) corresponding to this vertex.

\section{Expansions NeAr Zero CORRESPONDING to THE EDGE $\Gamma_{4}^{(1)}$}

3.1. Preliminary analysis. The edge $\Gamma_{4}^{(1)}$ is the left vertical edge, with the corresponding value $\omega=-1, r=0$, and the reduced equation

$$
\begin{aligned}
\hat{f}_{4}^{(1)}(x, y) \stackrel{\text { def }}{=} & 2 x^{2} y\left(y^{\prime}\right)^{2}-3 x^{2} y^{2}\left(y^{\prime}\right)^{2}-2 x y^{2} y^{\prime}+2 x y^{3} y^{\prime}-2 x^{2} y^{2} y^{\prime \prime} \\
& +2 x^{2} y^{3} y^{\prime \prime}-2 a y^{4}+2 c y^{4}+4 a y^{5}-2 a y^{6}=0 .
\end{aligned}
$$

The real normal cone is $\mathbf{U}_{4}^{(1)}=\{-\lambda(1,0), \lambda>0\}$.

The characteristic equation $\chi(\rho) \equiv 0$ corresponding to the bottom vertex $Q_{4}=(0,3)$ of the edge $\Gamma_{4}^{(1)}$ has the root $\rho=r+i \gamma$ with $r=0, \gamma \in \mathbb{R}, \gamma \neq 0$. The vector $\omega(1, \operatorname{Re} \rho)$ lies in $\mathbf{U}_{4}^{(1)}$. According to Case 1 of Theorem 1.5.1, there exist two families of exotic expansions $\mathcal{B}_{0}^{\tau}$ with $\tau=\operatorname{sgn} \gamma$ corresponding to the eigenvalue $\rho=i \gamma$.

According to Subsection 5.3 in Chapter 1, the reduced equation (2.3.1) has a solution in the form of the series

$$
y=c_{\rho} x^{\rho}+\sum_{k=2}^{\infty} \tilde{c}_{k-1} x^{k \rho},
$$

where the first term $y=c_{\rho} x^{\rho}$ of this series is the solution of the reduced equation (2.2.1) corresponding to the vertex $Q_{4}$; this equation was analyzed in Subsection 2.2 in Chapter 1. The characteristic equation (2.2.4) has a root $k_{1,2}=\rho$. According to Subsection 5.3 in Chapter 1 , the cone of the problem is $\mathcal{K}=\{\operatorname{Re} k=\operatorname{Re} \rho, \tau \operatorname{Im} k>$ $\tau \operatorname{Im} \rho$ \}. The numbers $k_{1,2}=\rho$ do not belong to the cone of the problem, so there are no critical numbers.

According to Subsection 2.2 in Chapter 1, the support of expansions of solutions (2.1.3) is

$$
\mathbf{K}=\{r=k \rho, k \geq 2 ; s=\rho+l \rho+m(1-\rho) ; l \geq 0 ; m \geq 1 ; k, l, m \in \mathbb{Z}\} .
$$

We obtain two families of exotic expansions:

$$
\mathcal{B}_{0}^{\tau}: \quad y=c_{\rho} x^{\rho}+\sum_{s} c_{s} x^{s}=x^{\rho}\left(c_{\rho}+\sum_{k=1}^{\infty} \tilde{c}_{k} x^{k \rho}\right)+\sum_{\operatorname{Re} s \geq 1} c_{s} x^{s},
$$

where $\rho \neq 0$ is an arbitrary purely imaginary constant, $r$ and $s$ run over the set (2.3.3), and the complex coefficients are as follows: $c_{\rho} \neq 0$ is an arbitrary constant, and $\tilde{c}_{k}$ and $c_{s}$ are uniquely determined constants.

The families $\mathcal{B}_{0}^{\tau}$ exist for $a \neq 0$. For more explicit formulas for the series (2.3.2), see (2.3.50) and (2.3.85).

Since $r=0$, we are looking for power solutions of the reduced equation (2.3.1) of the form $y=c_{0} \neq 0, c_{0}=$ const. Compute $c_{0}$. The defining equation is

$$
\tilde{f}\left(c_{0}\right) \stackrel{\text { def }}{=} c_{0}^{-3} \hat{f}_{4}^{(1)}\left(x, c_{0}\right) \stackrel{\text { def }}{=}-2 a c_{0}\left(c_{0}^{2}-2 c_{0}+1-c / a\right)=0 .
$$

The height of the edge equals three. Equation (2.3.5) has order three. It does always have the root $c_{0}=0$. According to Subsection 3.2 of Chapter 1, we should distinguish the case where equation $c_{0}^{2}-2 c_{0}+1-c / a=0$ has a zero root, a root at infinity, or where it has multiple roots. The equation never has a root at infinity, but it has a zero root $c_{0}=0$ for $a=c$ and a root $c_{0}=1$ of multiplicity 2 for $c=0$. Therefore, we consider separately three cases: $a \neq c \neq 0, a=c \neq 0$ and $a \neq 0, c=0$. 
Remark 2.3.1. For the main value of the square root of a complex number we take the principal value, so that the square root of a complex number $z$ can be written in the form $\pm \sqrt{z}$.

3.2. Expansions of solution in the case $a \neq c \neq 0$. First we compute power, powerlogarithmic, and exotic expansions of solutions of equation (2.1.4) corresponding to the edge $\Gamma_{4}^{(1)}$.

Equation (2.3.5) has two nonzero roots

$$
c_{0 i}=1+(-1)^{i} \sqrt{\frac{c}{a}}, \quad i=1,2 .
$$

The first variation is given by

$$
\begin{aligned}
\frac{\delta \hat{f}_{4}^{(1)}(x, y)}{\delta y}= & 2 x^{2}\left(y^{\prime}\right)^{2}+4 x^{2} y y^{\prime} \frac{d}{d x}-6 x^{2} y\left(y^{\prime}\right)^{2}-6 x^{2} y^{2} y^{\prime} \frac{d}{d x}-4 x y y^{\prime} \\
& -2 x y^{2} \frac{d}{d x}+6 x y^{2} y^{\prime}+2 x y^{3} \frac{d}{d x}-4 x^{2} y y^{\prime \prime}-2 x^{2} y^{2} \frac{d^{2}}{d x^{2}} \\
& +6 x^{2} y^{2} y^{\prime \prime}+2 x^{2} y^{3} \frac{d^{2}}{d x^{2}}-8 a y^{3}+8 c y^{3}+20 a y^{4}-12 a y^{5} .
\end{aligned}
$$

The linear differential operator is

$$
\begin{aligned}
\mathcal{L}(x)= & -2 x c_{0 i}^{2} \frac{d}{d x}+2 x c_{0 i}^{3} \frac{d}{d x}-2 x^{2} c_{0 i}^{2} \frac{d^{2}}{d x^{2}}+2 x^{2} c_{0 i}^{3} \frac{d^{2}}{d x^{2}}-8 a c_{0 i}^{3} \\
& +8 c c_{0 i}^{3}+20 a c_{0 i}^{4}-12 a c_{0 i}^{5} .
\end{aligned}
$$

The characteristic equation is

$$
\nu(k) \stackrel{\text { def }}{=} 2 c_{0 i}^{2}\left(k^{2}\left(c_{0 i}-1\right)-4 c_{0 i}(c-a)+10 a c_{0 i}^{2}-6 a c_{0 i}^{3}\right)=0 .
$$

Taking into account $c_{0 i}$ in (2.3.6) we see that the characteristic equation has two roots for each $i=1,2$ :

$$
k_{i}=\tau\left(\sqrt{2 c}+(-1)^{i} \sqrt{2 a}\right), \quad i=1,2, \tau= \pm 1 .
$$

According to Subsection 5.3 in Chapter 1 , for each $i=1,2$ there are two cones of the problem

$$
\mathcal{K}_{i}^{\tau}=\{s: \operatorname{Re} s \geq 0 \text { if } \operatorname{Re} s=0 \text {, then } \tau \operatorname{Im} s>0\} .
$$

Let us set $\theta_{i}=\sqrt{2 c}+(-1)^{i} \sqrt{2 a}$. For a fixed $i=1,2$ we will distinguish three cases.

Case $1 . \operatorname{Re} \theta_{i}=0$. In this case, for each of the cones of the problem $\mathcal{K}_{i}^{\tau}$ there is one critical number. Let, for example, $\operatorname{Im} \theta_{i}>0$. Then $\theta_{i} \in \mathcal{K}_{i}^{+}$and $-\theta_{i} \in \mathcal{K}_{i}^{-}$. The support of expansions of solutions is

$$
\mathbf{K}=\{s=l, l \in \mathbb{N}\} .
$$

Taking into account the critical numbers $k_{i} \stackrel{\text { def }}{=} \tau \theta_{i}$ we have two sets:

$$
\mathbf{K}\left(\tau \theta_{i}\right)=\left\{s=l+m \tau \theta_{i} ; l, m \in \mathbb{Z} ; l, m \geq 0 ; l+m>0\right\} .
$$

Since the critical numbers $\tau \theta_{i}$ do not belong to $\mathbf{K}$, according to Subsections 2.2 and 2.3 in Chapter 1, to these numbers there correspond two families of expansions

$$
\mathcal{B}_{i}^{\tau}: y=c_{0 i}+\sum_{s} c_{s i} x^{s}, \quad i=1,2,
$$

where $s \in \mathbf{K}\left(\tau \theta_{i}\right)$, and the complex coefficients are as follows: $c_{0 i}$ is as in (2.3.6), $c_{s i}$ with $s=\tau \theta_{i}$ is an arbitrary complex constant, and the other $c_{s i}$ are uniquely determined 
constants. The support $\mathbf{K}\left(\tau \theta_{i}\right)$ of each of the expansions (2.3.14) lies in the following quarter of the complex plane: $V_{+}^{\tau}=\{\operatorname{Re} s \geq 0, \tau \operatorname{Im} s \geq 0\}$.

Expansions (2.3.14) are exotic if $c_{s i}$ with $s=\tau \theta_{i}$ is a nonzero constant.

In the case $c_{s i}=0$ with $s=\tau \theta_{i}$, expansions (2.3.14) are expansions in integral powers of $x$. Families of such expansions will be denoted by $\mathcal{B}_{i}, i=1,2$.

In the case $c_{s i}=0$ with $s=\tau \theta_{i}$, expansion (2.3.14) (in integral powers of $x$ ) was known; see [54, $\S 46]$. According to Theorem 1.7.2 it converges for sufficiently small $|x|$.

In the case $c_{s i}=0$ with $s=\tau \theta_{i}$, the second approximation in expansions of solutions (2.3.14) is $y=c_{0 i}+c_{1 i} x$. Let us compute the coefficient $c_{1 i}$ which, according to Subsection 2.6 in Chapter 1, is determined from the equation $c_{1 i}=-\frac{b_{1 i}}{\nu(1)}$. The second approximation of equation (2.1.4) corresponding to the given edge is

$$
\begin{aligned}
\hat{\hat{f}}_{4}^{(1)}(x, y)= & -2(b-d) x y^{2}+2 x^{3} y^{\prime \prime} y+4(a+b-c-d) x y^{3}+6 x^{2} y^{\prime} y^{2} \\
& +2 x^{3} y^{\prime \prime} y^{2}-x^{3}\left(y^{\prime}\right)^{2}-2 x^{3}\left(y^{\prime}\right)^{2} y-2(4 a+b+c-d) x y^{4} \\
& -6 x^{2} y^{\prime} y^{3}-4 x^{3} y^{\prime \prime} y^{3}+6 x^{3}\left(y^{\prime}\right)^{2} y^{2}+4 a x y^{5} .
\end{aligned}
$$

The coefficient $b_{1 i}$ is given by

$b_{1 i} \stackrel{\text { def }}{=} x^{-1} \hat{\hat{f}}_{4}^{(1)}\left(x, c_{0 i}\right)=2 c_{0 i}^{2}\left(-b+d+2(a+b-c-d) c_{0 i}+(-4 a-b-c+d) c_{0 i}^{2}+2 a c_{0 i}^{3}\right)$.

According to (2.3.9),$\nu(1)=2 c_{0 i}^{2}\left(c_{0 i}-1-4 c_{0 i}(c-a)+10 a c_{0 i}^{2}-6 a c_{0 i}^{3}\right)$. Finally, taking into account (2.3.5) and (2.3.6) we obtain

$$
c_{1 i}=(-1)^{i} \sqrt{\frac{c}{a}} \frac{\left(\sqrt{a}+(-1)^{i} \sqrt{c}\right)^{2}+b-d}{1-2\left(\sqrt{a}+(-1)^{i} \sqrt{c}\right)^{2}} .
$$

Denote $k_{i}=\theta_{i}$ if $\operatorname{Re} \theta_{i}>0$ and $k_{i}=-\theta_{i}$ if $\operatorname{Re} \theta_{i}<0$.

Case 2. $\operatorname{Re} \theta_{i} \neq 0, \theta_{i} \notin \mathbb{Z}$. In this case both cones of problem $\mathcal{K}_{i}^{\tau}$ contain the common critical number $k_{i}$. Using $k_{i}$ we obtain that the support of expansions of solutions of (2.1.4) is

$$
\mathbf{K}\left(k_{i}\right)=\left\{s=l+m k_{i} ; l, m \in \mathbb{Z} ; l, m \geq 0 ; l+m>0\right\} .
$$

Since $k_{i}$ does not belong to the set $\mathbf{K}$ defined by formula (2.3.12), according to Subsection 2.2 in Chapter 1 we obtain a family of power expansions of solutions of (2.1.4) given by the formula

$$
\mathcal{B}_{i}: \quad y=c_{0 i}+\sum c_{s i} x^{s} \quad\left(\text { sum over } s \in \mathbf{K}\left(k_{i}\right)\right), \quad i=1,2,
$$

where $\mathbf{K}\left(k_{i}\right)$ is as in (2.3.17), and the complex coefficients are as follows: $c_{0 i}$ is defined by formula (2.3.6),$c_{k_{i} i}$ is arbitrary, and the other $c_{s i}$ are uniquely determined constants.

Expansion (2.3.18) was known before [54, $\S 46]$ only in the case where $c_{k_{i} i}=0$. By Theorem 1.7.2 it converges for sufficiently small $|x|$.

The second approximation of expansions of solutions (2.3.18) depends on the number $\operatorname{Re} k_{i}$. If $\operatorname{Re} k_{i}>1$, then the second approximation is $y=c_{0 i}+c_{1 i} x$, which is similar to the case $\operatorname{Re} \theta_{i}=0$. The coefficient $c_{1 i}$ is given by formula (2.3.16). If $0<\operatorname{Re} k_{i}<1$, then the second approximation of solutions is $y=c_{0 i}+c_{k_{i}} x^{k_{i}}$, where the coefficient $c_{k_{i} i}$ is arbitrary. If $\operatorname{Re} k_{i}=1$, then the second approximation is $y=c_{0 i}+c_{1 i} x+c_{k_{i} i} x^{k_{i}}$, where the coefficients are as follows: $c_{k_{i} i}$ is arbitrary and $c_{1 i}$ is defined by formula (2.3.16).

Case 3. $\theta_{i} \in \mathbb{Z} \backslash\{0\}$. In this case both cones of the problem contain the same critical number $k_{i}$. Since the number $k_{i}$ lies in the set $\mathbf{K}$ defined by formula (2.3.12), by 
Subsection 2.2 in Chapter 1 the family of expansions of solutions is

$$
\mathcal{B}_{i}: \quad y=c_{0 i}+\sum_{s=1}^{\infty} c_{s i}(\ln x) x^{s}, \quad i=1,2
$$

with the following coefficients: $c_{0 i}$ is defined by formula (2.3.6),$c_{k_{i} i}=\alpha_{k_{i} i}+\beta_{k_{i} i} \ln x$, $\alpha_{k_{i} i}$ is an arbitrary constant, the coefficient $\beta_{k_{i} i}$ is a uniquely determined constant, and the remaining $c_{s i}$ are uniquely determined polynomials in $\ln x$.

The second approximation of expansions of solutions (2.3.14) depends on the number $k_{i}$. If $k_{i}=1$, then the second approximation takes the form $y=c_{0 i}+c_{1 i} x$. According to Corollary 1.2.1 the coefficient $c_{1 i}$ is given by $c_{1 i}=\alpha_{1 i}+\beta_{1 i} \ln x$, where $\alpha_{1 i}$ is an arbitrary constant and $\beta_{1 i}$ is given by the formula $\beta_{1 i}=-\frac{b_{1 i}}{\nu^{\prime}(1)}$. The coefficient $b_{1 i}$ was computed earlier and is given by formula (2.3.15), $\nu^{\prime}(1)=4 c_{0 i}^{2}\left(c_{0 i}-1\right)$. Taking into account (2.3.6) we obtain

$$
c_{1 i}=\alpha_{1 i}+(-1)^{i} \sqrt{\frac{c}{a}} \frac{\left(\sqrt{a}+(-1)^{i} \sqrt{c}\right)^{2}+b-d}{2} \ln x .
$$

If $\left(\sqrt{a}+(-1)^{i} \sqrt{c}\right)^{2}+b-d=0$, then a compatibility condition is satisfied and the coefficients in expansion (2.3.19) are as follows: $c_{1 i}$ is an arbitrary constant, and the other $c_{s i}$ are uniquely determined constants.

Expansion (2.3.19) was known [54, §46] only in the case where the compatibility condition is satisfied and all coefficients $c_{s i}$ are constant. According to Theorem 1.7 .2 it converges for sufficiently small $|x|$. Expansions (2.3.14) are exotic, expansion (2.3.17) is power, and (2.3.19) is power-logarithmic.

Now according to Subsection 3.2 in Chapter 1 we compute nonpower solutions of equation (2.3.1) corresponding to the zero solution of the defining equation (2.3.5).

In the defining equation, we make a logarithmic transformation $\xi=\ln x$; under this transformation, $y^{\prime}$ and $y^{\prime \prime}$ are transformed by formulas (2.2.13). We obtain the equation

$$
\varphi(\xi, y) \stackrel{\text { def }}{=} \hat{f}_{4}^{(1)}(\xi, y)=2 \ddot{y} y^{2}(y-1)+\dot{y}^{2} y(2-3 y)+2(c-a) y^{4}+4 a y^{5}-2 a y^{6}=0 .
$$

The support $\mathbf{S}(\varphi)$, its convex hull, and faces $\Phi_{j}^{(d)}, d=0,1, j=1,2,3,4$, are shown in Figure 11(a), whereas the real normal cones $\mathbf{U}_{j}^{(d)}, d=0,1, j=1,2,3,4$ corresponding to the faces are shown in Figure 11 $(b)$.

Since $\xi=\ln x \rightarrow \infty$ as $x \rightarrow 0$, the cone of the problem is given by $\mathcal{K}=\left\{p_{1} \geq 0\right\}$ and $\omega=1$. Furthermore, $y \neq$ const. The cone of the problem has nonempty intersection with real normal cones $\mathbf{U}_{1}^{(0)}, \mathbf{U}_{2}^{(0)}, \mathbf{U}_{3}^{(0)}, \mathbf{U}_{1}^{(1)}, \mathbf{U}_{2}^{(1)}$. Consider the corresponding faces one by one.

The vertex $\Phi_{1}^{(0)}$. The corresponding reduced equation is

$$
\hat{\varphi}_{1}^{(0)}(\xi, y) \stackrel{\text { def }}{=}-2 \ddot{y} y^{2}+2 \dot{y}^{2} y=0 .
$$

The real normal cone is $\mathbf{U}_{1}^{(0)}=\left\{p_{2}<0, p_{2}<-2 p_{1}\right\}$. We are seeking solutions of equation (2.3.22) in the form $y=c_{r} \xi^{r}, c_{r} \neq 0$, where $c_{r}$ is an arbitrary constant. The characteristic equation $\chi(r) \stackrel{\text { def }}{=} r=0$ has a unique solution $r=0$. Since $P=\omega(1, r)=$ $(1,0) \notin \mathbf{U}_{1}^{(0)} \cap \mathcal{K}$, there are no appropriate solutions.

The vertices $\Phi_{2}^{(0)}$ and $\Phi_{3}^{(0)}$. The corresponding reduced equations are $\hat{\varphi}_{2}^{(0)}(\xi, y) \stackrel{\text { def }}{=}$ $2(c-a) y^{4}=0$ and $\hat{\varphi}_{3}^{(0)}(\xi, y) \stackrel{\text { def }}{=}-2 a y^{6}=0$. Since these equations are algebraic, according to Remark 1.1.1 there are no appropriate solutions.

The edge $\Phi_{1}^{(1)}$. The corresponding reduced equation is

$$
\hat{\varphi}_{1}^{(1)}(\xi, y) \stackrel{\text { def }}{=}-2 \ddot{y} y^{2}+2 \dot{y}^{2} y+2(c-a) y^{4}=0 .
$$



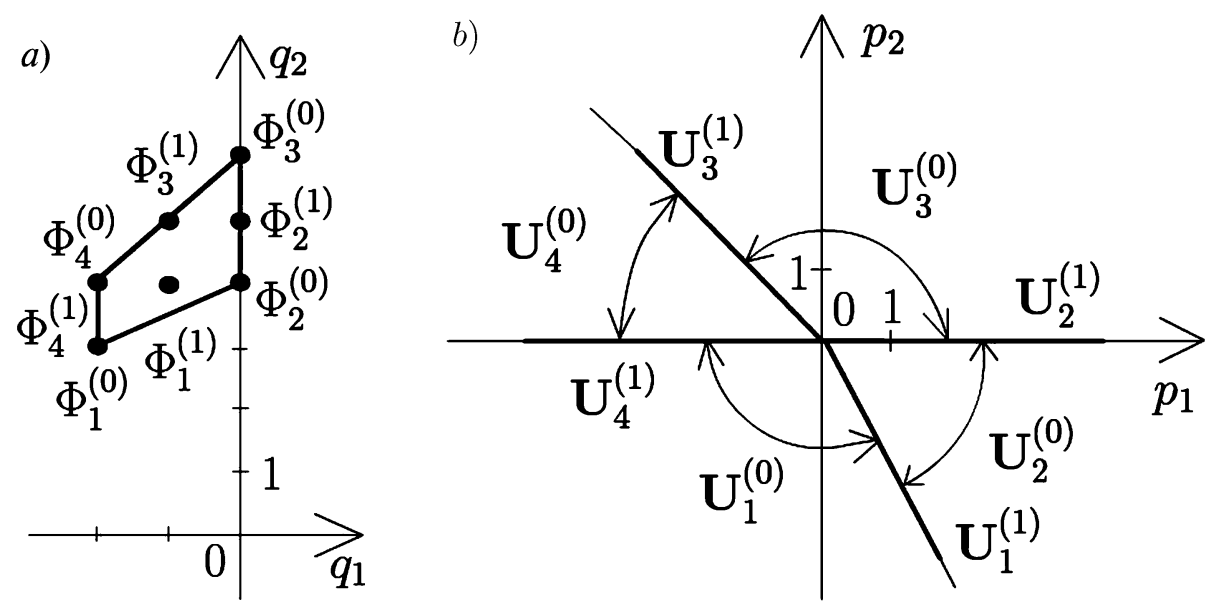

Figure 11. Data for equation (2.3.21): (a) the support, the polygon, and its faces; (b) the normal cones of faces.

The real normal cone is $\mathbf{U}_{1}^{(0)}=\{\lambda(1,-2), \lambda>0\}$. We are looking for solutions of equation (2.3.23) in the form $y=c_{-2} \xi^{-2}, c_{-2} \neq 0$. The defining equation $\tilde{\varphi}\left(c_{-2}\right) \stackrel{\text { def }}{=}$ $2 c_{-2}^{3}\left(c_{-2}(c-a)-2\right)=0$ has a nonzero solution

$$
c_{-2}=\frac{2}{c-a} \text {. }
$$

Let us compute critical numbers. The first variation is

$$
\frac{\delta \hat{\varphi}_{1}^{(1)}(\xi, y)}{\delta y} \stackrel{\text { def }}{=} 2 \dot{y}^{2}+4 y \dot{y} \frac{d}{d \xi}-4 y \ddot{y}-2 y^{2} \frac{d^{2}}{d \xi^{2}}+8(c-a) y^{3} .
$$

The linear differential operator is

$$
\mathcal{L}(\xi)=-2 c_{-2}^{2} \frac{1}{\xi^{6}}\left(\xi^{2} \frac{d^{2}}{d \xi^{2}}+8+4 \xi \frac{d}{d \xi}-4(c-a) c_{-2}\right) .
$$

The characteristic polynomial $\nu(k)=-2 c_{-2}^{2}\left(k^{2}+3 k\right)$ has two roots $k_{1}=0, k_{2}=-3$. The cone of the problem is

$$
\mathcal{K}=\{\operatorname{Re} k<-2 \text { or } \operatorname{Re} k=-2, \operatorname{Im} k \neq 0\} .
$$

The number $k_{2}=-3$ is the only number that belongs to the cone of the problem $\mathcal{K}$; i.e., $k_{2}$ is the only critical number. The support of the expansion of solutions is $\mathbf{K}=\{s=-2-2 l ; l>0\}$. Taking into account $k_{2}$ we see that the support is given by the formula

$$
\mathbf{K}\left(k_{2}\right)=\{s=-2-l ; l>0\} .
$$

The expansion of solutions is

$$
y=\frac{2}{c-a} \frac{1}{\xi^{2}}+\sum_{s=3}^{\infty} \frac{c_{-s}}{\xi^{s}} .
$$

Since $k_{2} \notin \mathbf{K}$, the compatibility condition is satisfied automatically; i.e., the complex coefficient $c_{-3}$ can be an arbitrary constant. By Theorem 1.7.2, the expansion (2.3.27) 
converges for sufficiently small $\frac{1}{\xi}$. Making the inverse substitution $\xi=\ln x$ in (2.3.27) we obtain the asymptotics of a solution of equation (2.1.4):

$$
y \stackrel{\text { def }}{=} \psi_{0}=\frac{2}{c-a} \frac{1}{\ln ^{2} x}+\frac{c_{-3}}{\ln ^{3} x}+\sum_{s=4}^{\infty} \frac{c_{-s}}{\ln ^{s} x},
$$

where the complex coefficients are as follows: $c_{-3}$ is an arbitrary constant, and the remaining $c_{-s}$ are uniquely determined constants.

Following Subsection 4.2 of Chapter 1, let us compute the critical numbers of the reduced solutions (2.3.28). The first variation is given by formula (2.3.7). Denote it by $\mathcal{M}(x, y)$. Let us make the logarithmic substitution $\xi=\ln x$ in $\mathcal{M}(x, y)$ and denote the derivative with respect to $\xi$ by a dot. In view of (2.2.13), for the operator $\mathcal{M}(x, y)$ we obtain

$$
\begin{aligned}
\mathcal{M}(x, y) \stackrel{\text { def }}{=} & 2 \dot{y}^{2}+4 y \dot{y} \frac{d}{d \xi}-6 y \dot{y}^{2}-6 y^{2} \dot{y} \frac{d}{d \xi}-4 y \dot{y}-2 y^{2} \frac{d}{d \xi}+6 y^{2} \dot{y} \\
& +2 y^{3} \frac{d}{d \xi}-4 y(\ddot{y}-\dot{y})-2 y^{2}\left(\frac{d^{2}}{d \xi^{2}}-\frac{d}{d \xi}\right)+6 y^{2}(\ddot{y}-\dot{y}) \\
& +2 y^{3}\left(\frac{d^{2}}{d \xi^{2}}-\frac{d}{d \xi}\right)-8 a y^{3}+8 c y^{3}+20 a y^{4}-12 a y^{5} \stackrel{\text { def }}{=} \mathcal{N}(\xi, y) .
\end{aligned}
$$

For solutions (2.3.27) we have $y=\frac{2 \xi^{-2}}{c-a}+\cdots$. Therefore, in the operator $\mathcal{N}$ the largest exponent $n$ in terms with $\xi^{n}$ is $n=-4$, and the terms with $\xi^{-4}$ form the operator $\mathcal{N}_{-4}=$ $-2 y^{2} \frac{d^{2}}{d \xi^{2}}$, where $y=\frac{2 \xi^{-2}}{c-a}$. The corresponding critical polynomial is $\nu(k)=\frac{-8 k^{2}}{(c-a)^{2}}$; this polynomial has the root $k=0$ of multiplicity 2 . The cone of the problem is $\mathcal{K}=$ $\{\operatorname{Re} k>0\}$. Since $k=0$ does not belong to the cone of the problem, there are no critical numbers. The support of the expansion of solutions has the form $\mathbf{K}=\{s=l, l \in \mathbb{N}\}$. By Theorem 1.4.1, there is a unique expansion for solutions of the original equation (2.1.4), which forms the family

$$
\mathcal{B}_{3}: \quad y=\psi_{0}+\sum_{\sigma=1}^{\infty} \psi_{\sigma} x^{\sigma}
$$

where $\psi_{0}$ is as in (2.3.28) and the $\psi_{\sigma}$ are series in decreasing powers of the logarithm.

By Theorem 1.4.2 the powers of the logarithms in $\psi_{\sigma}$ do not increase $-8 \sigma$.

Expansions (2.3.30) and (2.3.28) are new.

The vertex $\Phi_{2}^{(1)}$. The corresponding reduced equation is

$$
\hat{\varphi}_{2}^{(1)}(\xi, y) \stackrel{\text { def }}{=}-2 a y^{6}+4 a y^{5}+2(c-a) y^{4}=0 .
$$

This equation has only constant solutions $y=1+(-1)^{i} \sqrt{\frac{c}{a}}, i=1,2$. We do not need these solutions since they are solutions of the full equation (2.3.21) and coincide with the already studied solutions (2.3.6).

Now we solve equation (2.3.1) explicitly. To so this, we set $\dot{y}=p$ in (2.3.21) and view $p$ as a function in $y$. Then $\ddot{y}=\left(\frac{d p}{d y}\right) p$ and equation (2.3.21) takes the form

$$
2 \frac{d p}{d y} p y(y-1)+p^{2}(2-3 y)+2(c-a) y^{3}+4 a y^{4}-2 a y^{5}=0 .
$$

Taking $p^{2}=q$ we obtain a linear nonhomogeneous equation

$$
\frac{d q}{d y} y(y-1)+q(2-3 y)+2(c-a) y^{3}+4 a y^{4}-2 a y^{5}=0 .
$$


The corresponding homogeneous equation

$$
\frac{d q}{d y} y(y-1)+q(2-3 y)=0
$$

has a solution $q=C_{1} y^{2}(y-1)$, where $C_{1}$ is an arbitrary constant. Using the method of variation of this constant, we obtain from (2.3.32) the following equation for $C_{1}$ :

$$
(y-1)^{2} C_{1}^{\prime}+2(c-a)+4 a y-2 a y^{2}=0,
$$

i.e., $C_{1}^{\prime}=-\frac{2 c}{(y-1)^{2}}+2 a$. This equation has a solution $C_{1}=\frac{2 c}{y-1}+2 a y+C_{2}$, where $C_{2}$ is an arbitrary constant. Therefore,

$$
q \stackrel{\text { def }}{=} C_{1} y^{2}(y-1)=\left(\frac{2 c}{y-1}+2 a y+C_{2}\right) y^{2}(y-1)
$$

and

$$
\frac{d y}{d \xi} \stackrel{\text { def }}{=} p \stackrel{\text { def }}{=} \pm \sqrt{q}= \pm \sqrt{\left(\frac{2 c}{(y-1)}+2 a y+C_{2}\right) y^{2}(y-1)} .
$$

Integration of equation (2.3.33) depends on the value of $C_{2}$ and of parameters $a$ and c. Let us consider three cases of different values of $C_{2}$.

The case $C_{2}=2 c$. In this case equation (2.3.33) takes the form

$$
\frac{d y}{d \xi}= \pm \sqrt{2 a} y \sqrt{y^{2}+\left(\frac{c}{a}-1\right) y} .
$$

Let $t^{2}=1+(c / a-1) / y$. Then

$$
y=\frac{c / a-1}{t^{2}-1}
$$

and equation (2.3.34) takes the form $\frac{d t}{d \xi}= \pm \sqrt{2 a} / 2\left(\frac{c}{a}-1\right)$. Solutions of this equation are $t= \pm \sqrt{2 a} / 2(c / a-1)\left(\xi+C_{3}\right)$, where $C_{3}$ is an arbitrary constant. From (2.3.35) we obtain

$$
y \stackrel{\text { def }}{=} \psi_{0}=\frac{1}{\frac{c-a}{2}\left(\xi+C_{3}\right)^{2}-\frac{a}{c-a}} .
$$

As $\xi \rightarrow \infty$, formula (2.3.36) yields the Laurent expansion in powers of $\xi$ :

$$
y=\frac{2}{c-a} \frac{1}{\xi^{2}} \sum_{k=0}^{\infty}(-1)^{k}\left(\frac{C_{4}}{\xi}+\frac{C_{5}}{\xi^{2}}\right)^{k}
$$

where $C_{4} \stackrel{\text { def }}{=}-2 C_{3}$ is an arbitrary constant and $C_{5} \stackrel{\text { def }}{=}-C_{3}+\frac{2 a}{(c-a)^{2}}$. Taking into account that $\xi=\ln x$, we obtain an explicit form (2.3.36) of expansion (2.3.28).

The case $C_{2}=-2(\sqrt{a} \pm \sqrt{c})^{2}+2 c$. In this case equation (2.3.33) takes the form

$$
\frac{d y}{d \xi}= \pm \sqrt{2 a} y\left(y-\left(1 \mp \frac{c}{a}\right)\right) .
$$

The integral of this equation is

$$
\frac{y-\left(1+(-1)^{i} \sqrt{\frac{c}{a}}\right)}{y}=\exp \left( \pm \sqrt{2}\left(\sqrt{a}+(-1)^{i} \sqrt{c}\right) \xi+C_{6}\right),
$$

where $i=1,2$ and $C_{6}$ are arbitrary constants.

Taking into account that $\xi=\ln x$, formula (2.3.39) implies that

$$
y=\frac{1+(-1)^{i} \sqrt{c / a}}{1-C_{7} x^{ \pm \theta_{i}}}
$$


where $i=1,2, \theta_{i}=\sqrt{2}\left(\sqrt{a}+(-1)^{i} \sqrt{c}\right)$, and $C_{7}$ is an arbitrary constant.

Let, for example, $\operatorname{Re} \theta_{i} \geq 0, \theta_{i} \neq 0$. Then for $x \rightarrow 0$ the function (2.3.40) can be expanded in a series in powers of $C_{7} x^{\theta_{i}}$ :

$$
y=\left(1+(-1)^{i} \sqrt{\frac{c}{a}}\right) \sum_{k=0}^{\infty}(-1)^{k}\left(C_{7} x^{\theta_{i}}\right)^{k},
$$

where $i=1,2$ and $C_{7}$ is an arbitrary constant. Depending on the value of $\theta_{i}$ the series (2.3.41) is an explicit form of one of the expansions (2.3.14), (2.3.18), (2.3.19), and the function (2.3.40) is the sum of the expansion. form

The case $C_{2} \neq 2 c, C_{2} \neq-2(\sqrt{a} \pm \sqrt{c})^{2}+2 c$. Let us write equation (2.3.33) in the

$$
\frac{d y}{d \xi}= \pm \sqrt{2 a} \sqrt{y^{2}\left(y^{2}+\frac{C_{2}-2 a}{2 a} y+\frac{2 c-C_{2}}{2 a}\right)} .
$$

Equation $y^{2}+\frac{C_{2}-2 a}{2 a} y+\frac{2 c-C_{2}}{2 a}=0$ has two distinct nonzero roots $\alpha$ and $\beta$, and

$$
\alpha+\beta=-\frac{C_{2}-2 a}{2 a}, \quad \alpha \cdot \beta=\frac{2 c-C_{2}}{2 a} .
$$

The Euler substitution

$$
t^{2}=\frac{y-\beta}{y-\alpha}, \quad y=\frac{\alpha t^{2}-\beta}{t^{2}-1}
$$

transforms equation (2.3.33) into the form

$$
\frac{ \pm 2 d t}{\sqrt{2 a}\left(\alpha t^{2}-\beta\right)}=d \xi
$$

The integral of this equation is

$$
\pm \frac{1}{\sqrt{2 a \alpha \beta}} \ln \frac{t-\sqrt{\beta / \alpha}}{t+\sqrt{\beta / \alpha}}=\xi+C_{8},
$$

where $C_{8}$ is an arbitrary constant. Taking into account (2.3.43) and (2.3.45) we obtain

$$
\frac{t-\sqrt{\beta / \alpha}}{t+\sqrt{\beta / \alpha}}=C_{9} \exp \left( \pm \sqrt{2 c-C_{2}} \xi\right)
$$

where $C_{9}$ is an arbitrary constant. Hence,

$$
t=-\sqrt{\frac{\beta}{\alpha}}\left(1-\frac{2}{1-C_{9} \exp \left( \pm \sqrt{2 c-C_{2}} \xi\right)}\right) .
$$

Set

$$
\varphi=C_{9} \exp \left( \pm \sqrt{2 c-C_{2}} \xi\right) .
$$

Then (2.3.44) and (2.3.46) imply that

$$
y=\frac{4 \alpha \beta}{\beta(\sqrt{1 / \varphi}+\sqrt{\varphi})^{2}-\alpha(\sqrt{1 / \varphi}-\sqrt{\varphi})^{2}} .
$$

Set $2 i \psi=\ln \varphi \stackrel{\text { def }}{=} \pm \sqrt{2 c-C_{2}}\left(\xi+C_{10}\right)$, where $C_{10}$ is an arbitrary constant. Taking into account that $\xi=\ln x$ we have

$$
\psi= \pm i \frac{\sqrt{2 c-C_{2}}}{2}\left(\ln x+C_{10}\right)
$$


Then (2.3.48) takes the form

$$
y=\frac{\alpha \beta}{\beta \cos ^{2} \psi+\alpha \sin ^{2} \psi} .
$$

According to (2.3.47), $y$ can be expanded in a series in powers of $\varphi=\left(C_{11} x\right)^{ \pm \sqrt{2 c-C_{2}}}$, where $C_{11}$ is an arbitrary constant.

If $2 c-C_{2}$ is a real negative number, then $i \sqrt{2 c-C_{2}} \stackrel{\text { def }}{=} 2 \gamma$ is real. In this case $\psi=\gamma \ln \left(C_{11} x\right)$ and, according to (2.3.43) and (2.3.49),

$$
y=\frac{2 c-C_{2}}{2 a} \frac{1}{\beta \cos ^{2}\left[\ln \left(C_{11} x\right)^{\gamma}\right]+\alpha \sin ^{2}\left[\ln \left(C_{11} x\right)^{\gamma}\right]} .
$$

Formula (2.3.49) shows that $y$ can be expanded in a series in integral powers of $x^{2 \gamma i}$, thus yielding the series (2.3.2) in purely imaginary powers of $x$. According to (2.3.48), $y^{-1}$ is a sum of the form $c_{1} x^{2 i \gamma}+c_{0}+c_{-1} x^{-2 i \gamma}$, where $c_{0}$ and $c_{ \pm 1}$ are complex constants independent of the sign of $\gamma$. On the other hand, in families $\mathcal{B}_{0}^{\tau}$ we have $\tau=\operatorname{sgn} \gamma= \pm 1$ (see (2.3.4)). According to Subsection 5.4 in Chapter 1, the families $\mathcal{B}_{0}^{+}$and $\mathcal{B}_{0}^{-}$have the common family of inverse expansions $y^{-1}$.

The series (2.3.2) with $\rho= \pm \sqrt{2 c-C_{2}}, 2 c-C_{2} \in \mathbb{R}, 2 c-C_{2}<0$, is the function (2.3.50), which is a solution of the reduced equation (2.3.1) corresponding to the edge $\Gamma_{4}^{(1)}$.

Moreover, for the vertex $\Gamma_{4}^{(0)}$ the subsum of the sum (2.2.6) corresponding to $m=0$ in (2.2.5) is the solution (2.3.49) of the reduced equation (2.3.1) corresponding to the edge $\Gamma_{4}^{(1)}$.

3.3. Expansions of solution for $a=c \neq 0$. First we compute power, power-logarithmic, and exotic expansions of solutions of equation (2.1.1) corresponding to the edge $\Gamma_{4}^{(1)}$. In this case the defining equation (2.3.5) has two zero solutions and one nonzero solution $c_{02}=2$ from (2.3.6). To this nonzero solution there correspond two eigenvalues $k_{2}= \pm 2 \sqrt{2 a}$ from (2.3.10). For $\theta_{2}$ we take the value $k_{2}$ for which $\operatorname{Re} k_{2} \geq 0$.

To the value $c_{02}$ there correspond families of expansions $\mathcal{B}_{2}$ or $\mathcal{B}_{2}^{\tau}$; for these families, all formulas correspond to power, power-logarithmic, or exotic expansions from the case $a \neq c \neq 0$. Similarly to that case, here we also have three possibilities depending on the value of $\theta_{2}$. Namely, Case $1\left(\operatorname{Re} \theta_{2}=0\right.$, and expansion of solutions is determined by formula (2.3.14) $)$, Case $2\left(\operatorname{Re} \theta_{2} \neq 0, \theta_{2} \notin \mathbb{Z}\right.$, and expansion of solutions is determined by formula (2.3.18) $)$, and Case $3\left(\theta_{2} \in \mathbb{Z} \backslash\{0\}\right.$, and expansion of solutions is determined by formula (2.3.19) $)$.

Now we compute nonpower solutions of equation (2.3.1) corresponding to the multiplicity 2 zero solution of the defining equation (2.3.5). In this case equation (2.3.21) takes the form

$$
\phi(\xi, y) \stackrel{\text { def }}{=} \hat{f}_{4}^{(1)}(\xi, y) \stackrel{\text { def }}{=} 2 \ddot{y} y^{2}(y-1)+\dot{y}^{2} y(2-3 y)-2 a y^{6}+4 a y^{5}=0 .
$$

The support $\mathbf{S}(\phi)$, its convex hull, faces $\Phi_{j}^{(d)}, d=0,1, j=1,2,3,4$, are shown in Figure 12] $(a)$, real normal cones $\mathbf{U}_{j}^{(d)}, d=0,1, j=1,2,3,4$ corresponding to faces are shown in Figure $12(b)$.

The cone of the problem is given by $\mathcal{K}=\left\{p_{1} \geq 0\right\}$, i.e., $\omega=1$. Furthermore, $y \neq$ const. The cone of the problem has nontrivial intersections with the real normal cones $\mathbf{U}_{1}^{(0)}$, $\mathbf{U}_{2}^{(0)}, \mathbf{U}_{3}^{(0)}, \mathbf{U}_{1}^{(1)}, \mathbf{U}_{2}^{(1)}$. Consider the corresponding faces separately.

The reduced equation corresponding to the vertex $\Phi_{1}^{(0)}$ is (2.3.22). The real normal cone is $\mathbf{U}_{1}^{(0)}=\left\{p_{1}<0, p_{2}<-p_{1}\right\}$. The vector $P=(1,0)$, obtained earlier in the case 

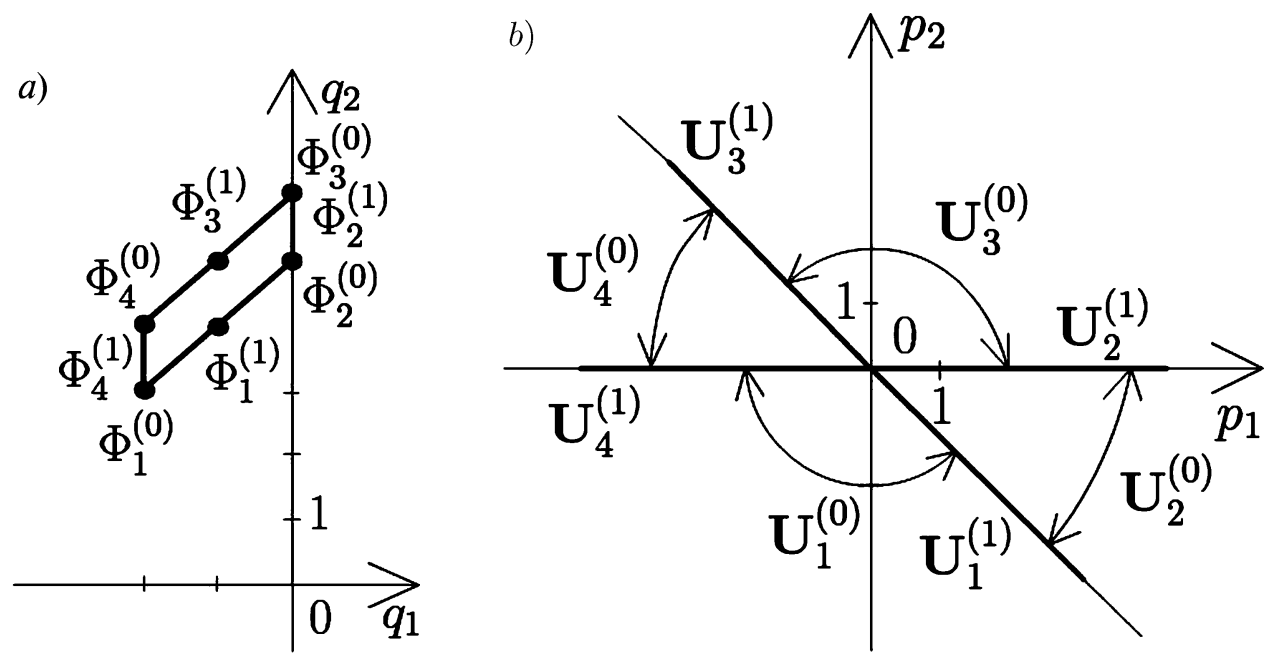

FiguRE 12. For equation (2.3.51) we show (a) the support, polygon, and its faces; $(b)$ normal vectors to these faces.

$a \neq c$, does not belong to the normal cone $\mathbf{U}_{1}^{(0)}$. Therefore, there are no appropriate solutions.

The reduced equations corresponding to the vertices $\Phi_{2}^{(0)}$ and $\Phi_{3}^{(0)}$ are $\hat{\phi}_{2}^{(0)}(\xi, y) \stackrel{\text { def }}{=}$ $4 a y^{5}=0$ and $\hat{\phi}_{3}^{(0)}(\xi, y) \stackrel{\text { def }}{=}-2 a y^{6}=0$ respectively. These equations are algebraic, and according to Remark 1.1.1 these equations do not yield appropriate solutions.

The reduced equation corresponding to the edge $\Phi_{1}^{(1)}$ is

$$
\hat{\phi}_{1}^{(1)}(\xi, y) \stackrel{\text { def }}{=}-2 \ddot{y} y^{2}+2 \dot{y}^{2} y+4 a y^{5}=0 .
$$

The real normal cone is $\mathbf{U}_{1}^{(0)}=\{\lambda(1,-1), \lambda>0\}$. We look for solutions of (2.3.52) of the form $y=c_{-1} \xi^{-1}, c_{-1} \neq 0$. The defining equation $\tilde{\phi}\left(c_{-1}\right) \stackrel{\text { def }}{=}-2 c_{-1}^{3}\left(2 a c_{-1}^{2}-1\right)=0$ has two nonzero solutions

$$
c_{-1}=\frac{(-1)^{j}}{\sqrt{2 a}}, \quad j=1,2
$$

Let us compute critical numbers. The first variation is

$$
\frac{\delta \hat{\phi}_{1}^{(1)}(\xi, y)}{\delta y} \stackrel{\text { def }}{=} 2 \dot{y}^{2}+4 y \dot{y} \frac{d}{d \xi}-4 y \ddot{y}-2 y^{2} \frac{d^{2}}{d \xi^{2}}+20 y^{4} .
$$

The linear differential operator is

$$
\mathcal{L}(\xi)=-2 c_{-1}^{2} \xi^{-4}\left(\xi^{2} \frac{d^{2}}{d \xi^{2}}+2 \xi \frac{d}{d \xi}+3-10 a c_{-1}^{2}\right) .
$$

The characteristic polynomial $\nu(k)=-2 c_{-1}^{2}\left(k^{2}+k-2\right)$ has two roots, $k_{1}=1, k_{2}=-2$. The cone of the problem is

$$
\mathcal{K}=\{\operatorname{Re} k<-1 \text { or } \operatorname{Re} k=-1, \operatorname{Im} k \neq 0\} .
$$

The number $k_{2}=-2$ belongs to the cone of the problem $\mathcal{K}$; i.e., $k_{2}$ is the only critical number. The initial support of the expansion of solutions is $\mathbf{K}=\{s=-1-2 l ; l>0\}$. Taking into account $k_{2}$, the support of the expansion of solutions becomes

$$
\mathbf{K}\left(k_{2}\right)=\{s=-1-l ; l>0\} .
$$


Expansions of solutions are

$$
y=(-1)^{j} \frac{\xi^{-1}}{\sqrt{2 a}}+\sum_{s=2}^{\infty} c_{-s j} \xi^{-s}, \quad j=1,2 .
$$

Since $k_{2} \notin \mathbf{K}$, the compatibility condition is satisfied automatically; i.e., the complex coefficient $c_{-2 j}$ is an arbitrary constant.

Making the inverse substitution $\xi=\ln x$ in (2.3.56) we obtain two families of asymptotics of solutions of equation (2.1.4),

$$
\mathcal{F}_{j}: \quad y \stackrel{\text { def }}{=} \psi_{0 j}=(-1)^{j} \frac{1}{\sqrt{2 a}} \frac{1}{\ln x}+\frac{c_{-2 j}}{\ln ^{2} x}+\sum_{s=3}^{\infty} \frac{c_{-s j}}{\ln ^{s} x}, \quad j=1,2,
$$

where the complex coefficients are as follows: $c_{-2 j}$ is an arbitrary constant, and the remaining $c_{-s j}$ are uniquely determined constants.

By Theorem 1.7.2 the expansions (2.3.56) converge for sufficiently small $\frac{1}{\xi}$.

Following Subsection 4.2 of Chapter 1, let us compute the critical numbers of the reduced solutions (2.3.57). The operator $\mathcal{N}(\xi, y)$ is determined by formula (2.3.29). For solutions of (2.3.56) we have $y=(-1)^{j} \frac{\xi^{-1}}{\sqrt{2 a}}+\cdots$. Therefore among all terms with $\xi^{n}$ in the operator $\mathcal{N}$ the highest exponent $n$ is $n=-2$, and the terms with $\xi^{-2}$ form the operator $\mathcal{N}_{-2}=-2 y^{2} \frac{d^{2}}{d \xi^{2}}$, where $y=(-1)^{j} \frac{\xi^{-1}}{\sqrt{2 a}}$. The corresponding characteristic polynomial $\nu(k)=-\frac{k^{2}}{2 a}$ has a double root $k=0$. The cone of the problem is $\mathcal{K}=$ $\{\operatorname{Re} k>0\}$. Since $k=0$ does not belong to the cone of the problem, there are no critical numbers. The support of the expansion of solutions is of the form $\mathbf{K}=\{s=l, l \in \mathbb{N}\}$. By Theorem 1.4.1 solutions of the original equation (2.1.4) have unique expansions forming the families

$$
\mathcal{B}_{3+j}: \quad y=\psi_{0 j}+\sum_{\sigma=1}^{\infty} \psi_{\sigma j} x^{\sigma}, \quad j=1,2,
$$

where $\psi_{0 j}$ is from (2.3.57) and $\psi_{\sigma j}$ are the series in decreasing powers of logarithms.

By Theorem 1.4.2 powers of logarithms in $\psi_{\sigma j}$ do not exceed $-4 \sigma$.

Expansions (2.3.58) and (2.3.57) are new.

The reduced equation corresponding to the edge $\Phi_{2}^{(1)}$ is $\hat{\phi}_{2}^{(1)}(\xi, y) \stackrel{\text { def }}{=}-2 a y^{6}+4 a y^{5}=0$. It has the constant solution $y=2$. This solution is not appropriate since it is a solution of the full equation (2.3.51) and coincides with the solution $c_{02}$ we have studied at the beginning of this subsection.

Let us find explicit solutions of equation (2.3.1) for $a=c \neq 0$. To do this, we consider equation (2.3.33).

Integration of equation (2.3.33) is carried out differently in different cases depending on the values of $C_{2}$ and $a$. We consider three cases of the value of $C_{2}$.

Case $C_{2}=2 a$. In this case equation (2.3.33) takes the form

$$
\frac{d y}{d \xi}= \pm \sqrt{2 a} y^{2} .
$$

Equation (2.3.34) has a solution

$$
y= \pm \frac{1}{\sqrt{2 a} \xi+C_{3}},
$$

where $C_{3}$ is an arbitrary constant. 
Recall that $x \rightarrow 0$, i.e., $\xi \stackrel{\text { def }}{=} \ln x \rightarrow \infty$ ( $x$ and $\xi$ are complex). Then (2.3.60) can be expanded in the Laurent series in $\xi$ :

$$
y= \pm \frac{1}{\sqrt{2 a}} \frac{1}{\xi} \sum_{k=0}^{\infty}(-1)^{k} \frac{1}{\left(C_{4} \xi\right)^{k}}
$$

where $C_{4} \stackrel{\text { def }}{=} \pm C_{3} / \sqrt{2 a}$ is an arbitrary constant.

Taking into account that $\xi=\ln x$, we obtain the following explicit form of expansion (2.3.57):

$$
y \stackrel{\text { def }}{=} \psi_{0 j}= \pm \frac{1}{\sqrt{2 a}} \frac{1}{\ln x} \sum_{k=0}^{\infty}(-1)^{k} \frac{1}{\left(C_{4} \ln x\right)^{k}} \stackrel{\text { def }}{=} \pm \frac{1}{\sqrt{2 a} \ln x+C_{3}} .
$$

Case $C_{2}=-6 a$. In this case equation (2.3.33) has a solution

$$
y=\frac{2}{1-C_{5} x^{\theta_{2}}},
$$

where $i=1,2, \theta_{2}=2 \sqrt{2 a}$, and $C_{5}$ is an arbitrary constant.

For $\operatorname{Re} \theta_{2} \geq 0, \theta_{2} \neq 0$ and $x \rightarrow 0$ the function (2.3.63) can be expanded in a series in powers of $C_{5} x^{\theta_{2}}$ :

$$
y=2 \sum_{k=0}^{\infty}(-1)^{k}\left(C_{5} x^{\theta_{2}}\right)^{k},
$$

where $i=1,2$ and $C_{5}$ is an arbitrary constant. Depending on the value of $\theta_{2}$ the series (2.3.64) is an explicit form of expansions (2.3.14), (2.3.18), or (2.3.19) and the function $(2.3 .63)$ is the sum of these expansions.

Case $C_{2} \neq 2 a, C_{2} \neq-6 a$. In this case equation (2.3.33) has the solution (2.3.49) coming from the case $a \neq c \neq 0$.

3.4. Expansion of solutions for $a \neq 0, c=0$. First we compute power, powerlogarithmic, and exotic expansions of solutions of equation (2.1.1) corresponding to the edge $\Gamma_{4}^{(1)}$. Equation (2.3.5) has the double root $c_{0}=1$. According to (2.3.8), for $a \neq 0, c=0$, the corresponding linear operator satisfies $\mathcal{L}(x) \equiv 0$.

To analyze equation (2.1.4) in this case, make the substitution $y=1+u$. We obtain the equation

$$
\begin{aligned}
g(x, u) \stackrel{\text { def }}{=} & f(x, u+1) \stackrel{\text { def }}{=}\left((2 u+1) u^{\prime 2}-2\left(u+u^{2}\right) u^{\prime \prime}\right) x^{5} \\
& +\left(\left(-3-8 u-3 u^{2}\right) u^{\prime 2}-2\left(u+u^{2}\right) u^{\prime}+2\left(4 u^{2}+u^{3}+3 u\right) u^{\prime \prime}\right) x^{4} \\
& +\left(\left(10 u+6 u^{2}+3\right) u^{\prime 2}+2\left(5 u^{2}+2 u^{3}+3 u\right) u^{\prime}-2\left(5 u^{2}+3 u+2 u^{3}\right) u^{\prime \prime}-2 b u^{2}\right) x^{3} \\
+ & \left(\left(-3 u^{2}-4 u-1\right) u^{\prime 2}-6\left(2 u^{2}+u+u^{3}\right) u^{\prime}+2\left(u^{3}+2 u^{2}+u\right) u^{\prime \prime}\right. \\
& \quad-2(d+a) u^{4}-4(a+d-b) u^{3}+\left(2\left(u^{3}+2 u^{2}+u\right) u^{\prime}\right. \\
& \left.\quad+4 a u^{5} 2(6 a-b+d) u^{4}-4(b-3 a-d) u^{3}-2(b-d-2 a) u^{2}\right) x \\
& -8 a u^{3}-12 a u^{4}-2 a u^{2}-8 a u^{5}-2 a u^{6}=0 .
\end{aligned}
$$

The support $\mathbf{S}(g)$, its convex hall $\boldsymbol{\Gamma}(g)$, and the faces $G_{i}^{(d)}, d=0,1, i=1,2,3,4$, are shown in Figure $13(a)$. Real normal cones $\mathbf{U}_{i}^{(d)}, d=0,1, i=1,2,3,4$, are shown in Figure $13(b)$. 

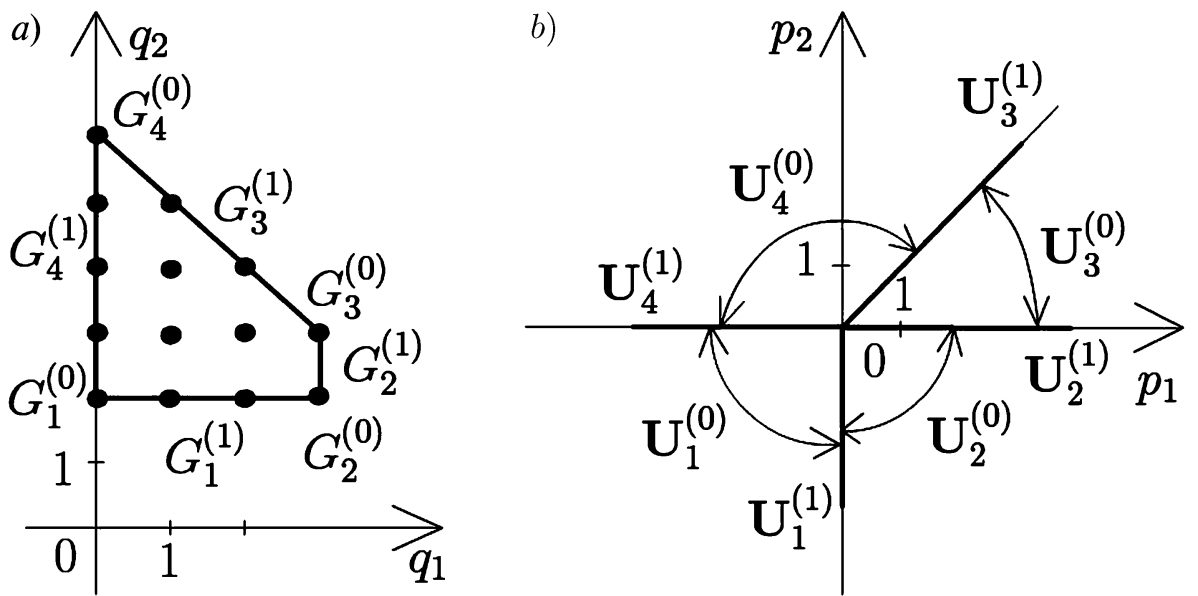

FIGURE 13. Data for equation (2.3.65): (a) the support, the polygon, and the faces; $(b)$ the corresponding normal cones.

Equation (2.3.65) has the trivial solution $u=0$ of multiplicity 2. The corresponding exceptional solution of (2.1.4) is

$$
\mathcal{I}_{2}: \quad y=1
$$

By Theorem 2.1.4 this solution is a multiplicity one exceptional solution of the initial equation (2.1.1); the double multiplicity of $y=1$ resulted from the multiplication of equation (2.1.1) by the factor $2 x^{2}(x-1)^{2} y(y-1)(y-x)$ containing the subfactor $(y-1)$.

The cone of the problem is $\mathcal{K}=\left\{p_{1} \leq 0, p_{2}<0\right\}$, so that $\omega=-1$. Furthermore, $u(x) \neq$ const. Real normal cones with nonempty intersection with the cone of the problem are $\mathbf{U}_{1}^{(0)}$ and $\mathbf{U}_{1}^{(1)}$.

The reduced equation corresponding to the vertex $G_{1}^{(0)}$ is

$$
\hat{g}_{1}^{(0)}(x, u) \stackrel{\text { def }}{=} 2 u^{\prime \prime} u x^{2}-u^{\prime 2} x^{2}+2 u^{\prime} u x-2 a u^{2}=0 .
$$

The real normal cone is $\mathbf{U}_{1}^{(0)}=\left\{p_{1}<0, p_{2}<0\right\}$. The first approximation to the solution has the $u=c_{\rho} x^{\rho}$, where $c_{\rho}$ is an arbitrary nonzero constant. The exponent $\rho$ is determined from the characteristic equation

$$
\chi(\rho) \stackrel{\text { def }}{=} \rho^{2}-2 a=0,
$$

which yields $\rho_{1,2}= \pm \sqrt{2 a}$.

According to Subsection 5.3 in Chapter 1, the complex normal cone is $\breve{\mathbf{U}}_{1}^{(0)}=-(1, \rho)$, where

$$
\rho: \quad \rho \in \mathbb{C}, \operatorname{Re} \rho>0 .
$$

Let us compute critical numbers. The first variation is

$$
\frac{\partial \hat{g}_{1}^{(0)}(x, u)}{\partial u}=2 u^{\prime \prime} x^{2}+2 \frac{d^{2}}{d x^{2}} u x^{2}-2 u^{\prime} \frac{d}{d x} x^{2}+2 u^{\prime} x+2 \frac{d}{d x} u x-4 a u .
$$

The linear differential operator is given by the formula

$$
\mathcal{L}(x)=2 c_{\rho} x^{\rho}\left(\rho(\rho-1)+\frac{d^{2}}{d x^{2}} x^{2}-\rho \frac{d}{d x} x+\rho+\frac{d}{d x} x-2 a\right) .
$$

The characteristic equation $\nu(k) \stackrel{\text { def }}{=} 2 c_{\rho} k(k-\rho)=0$ has two roots $k_{1}=0, k_{2}=\rho$. 
According to Subsection 5.3 of Chapter 1 , the cone of the problem is $\mathcal{K}=\{\operatorname{Re} k>\operatorname{Re} \rho$ or $\operatorname{Re} k=\operatorname{Re} \rho,|\operatorname{Im} k|>|\operatorname{Im} \rho|, \operatorname{sgn}(\operatorname{Im} k)=\operatorname{sgn}(\operatorname{Im} \rho)\}$. Since $k_{1,2} \notin \mathcal{K}$, there are no critical numbers.

The support of expansions of solutions is

$$
\mathbf{K}=\{s=\rho+l \rho+m ; l, m \geq 0 ; l+m>0 ; l, m \in \mathbb{Z}\} .
$$

The expansion of solution has the form

$$
u=c_{\rho} x^{\rho}+\sum c_{s} x^{s} \quad(\text { sum over } s \in \mathbf{K}) .
$$

The support of expansion (2.3.70) in the complex plane $s$ lies in the sector with vertex at $\rho$ spanned by the points $s=2 \rho$ and $s=\rho+1$.

After the inverse substitution $y=1+u$, expansions (2.3.70) yield the family

$$
\mathcal{B}_{6}: \quad y=1+c_{\rho} x^{\rho}+\sum_{s} c_{s} x^{s},
$$

where $\rho= \pm \sqrt{2 a}$ satisfies (2.3.68), $s$ runs over the set (2.3.69), and the complex coefficients are as follows: $c_{\rho}$ is a nonzero constants, and all $c_{s}$ are uniquely determined constants. According to Theorem 1.7.2 expansion (2.3.71) converges for sufficiently small $|x|$. In the case $\operatorname{Re} \rho=0$ expansions (2.3.71) are exotic. We denote the corresponding families by $\mathcal{B}_{6}^{\tau}$ where $\tau=\operatorname{sgn}(\operatorname{Im} \rho)$.

The families $\mathcal{B}_{6}$ and $\mathcal{B}_{6}^{\tau}$ are new. If $\sqrt{2 a} \in \mathbb{N}$, then, by (2.3.69) all exponents $s$ are integers.

Let us compute the second approximation of solutions (2.3.70) for $\operatorname{Re} \rho>0$.

In case $\operatorname{Re} \rho>1$ the second approximation is $u=c_{\rho} x^{\rho}+c_{\rho+1} x^{\rho+1}$. The second approximation to equation (2.3.65) is

$$
\hat{\hat{g}}_{1}^{(0)}(x, u)=3{u^{\prime}}^{2} x^{3}-6 u u^{\prime \prime} x^{3}-6 u u^{\prime} x^{2}+2 x u^{2}(2 a-b+d) .
$$

Furthermore, $c_{\rho+1}=-b_{\rho+1} / \nu(\rho+1)$, where $b_{\rho+1} \stackrel{\text { def }}{=} x^{-2 \rho-1} \hat{\hat{g}}_{1}^{(0)}\left(x, c_{\rho} x^{\rho}\right)=2 c_{\rho}^{2}(d-b-a)$, $\nu(\rho+1)=2 c_{\rho}(\rho+1)$; hence

$$
c_{\rho+1}=c_{\rho} \frac{a+b-d}{\rho+1} .
$$

In case $0<\operatorname{Re} \rho<1$ the second approximation to solutions is $u=c_{\rho} x^{\rho}+c_{2 \rho} x^{2 \rho}$. The second approximation to equation (2.3.65) is

$$
\hat{\hat{g}}_{1}^{(0)}(x, u)=-4 u u^{\prime 2} x^{2}+4 u^{2} u^{\prime \prime} x^{2}+4 u^{2} u^{\prime} x-8 a u^{3} .
$$

Furthermore, $c_{2 \rho}=-\frac{b_{2 \rho}}{\nu(2 \rho)}$, where $b_{2 \rho} \stackrel{\text { def }}{=} x^{-3 \rho} \hat{\hat{g}}_{1}^{(0)}\left(x, c_{\rho} x^{\rho}\right)=-8 a c_{\rho}^{3}, \nu(2 \rho)=8 a c_{\rho}$. Therefore,

$$
c_{2 \rho}=c_{\rho}^{2} .
$$

In case $\operatorname{Re} \rho=1, \operatorname{Im} \rho \neq 0$ the second approximation of solutions is $u=c_{\rho} x^{\rho}+$ $c_{\rho+1} x^{\rho+1}+c_{2 \rho} x^{2 \rho}$, where the coefficients $c_{\rho+1}$ and $c_{2 \rho}$ are given by formulas (2.3.72) and (2.3.73) respectively.

In case $\rho=1$, i.e., $a=\frac{1}{2}$, the second approximation to solutions is $u=c_{1} x+c_{2} x^{2}$, where $c_{1}$ is an arbitrary constant and

$$
c_{2}=c_{1} \frac{a+b-d+2 c_{1}}{2}
$$

is the sum of (2.3.72) and (2.3.73). 
Let us compute nonpower solutions of equation (2.3.66) in case they exist. Transform this equation as follows:

$$
h(x, u) \stackrel{\text { def }}{=} u^{-2} \hat{g}_{1}^{(0)}(x, u) \stackrel{\text { def }}{=} 2 \frac{u^{\prime \prime}}{u} x^{2}-\frac{u^{\prime 2}}{u^{2}} x^{2}+2 \frac{u^{\prime}}{u} x-2 a=0,
$$

so that $\mathbf{S}(h)=\{0\}$. Equation $h(x, u)=0$ contains a nonzero constant $-2 a$,

$$
h^{*}=2 \frac{u^{\prime \prime}}{u} x^{2}-\frac{u^{\prime 2}}{u^{2}} x^{2}, \quad \operatorname{coef}\left(h^{*}\right)=2-1=1 \neq 0 .
$$

Since $a \neq 0$, equation (2.3.67) does not have multiple roots, i.e., by Theorems 1.3.5 and 1.3.6 there are no nonpower solutions of equation (2.3.66). Therefore, there are no complicated solutions of equation (2.1.4) corresponding to the vertex $G_{1}^{(0)}$.

The reduced equation corresponding to the edge $G_{1}^{(1)}$ is

$$
\begin{aligned}
\hat{g}_{1}^{(1)}(x, u) \stackrel{\text { def }}{=} & 2 u^{\prime \prime} u x^{2}-u^{\prime 2} x^{2}+2 u^{\prime} u x-2 a u^{2}-6 u^{\prime \prime} u x^{3}+3{u^{\prime}}^{2} x^{3}-6 u^{\prime} u x^{2} \\
& -2(b-d-2 a) u^{2} x 6 u^{\prime \prime} u x^{4}-30{u^{\prime}}^{2} x^{4}+6 u^{\prime} u x^{3} \\
& -2(d+a-2 b) u^{2} x^{2}-2 u^{\prime \prime} u x^{5}+{u^{\prime}}^{2} x^{5}-2 u^{\prime} u x^{4}-2 b u^{2} x^{3}=0 .
\end{aligned}
$$

The edge $G_{1}^{(1)}$ is horizontal. Equation (2.3.77) has no power solutions, i.e., no power or power-logarithmic expansions of solutions. It can happen that equation (2.3.77) has nonpower solutions. The reduced equation corresponding to the left vertex $G_{1}^{(0)}=(0,2)$ of this edge is (2.3.66). Since for both these equations (2.3.66) and (2.3.77) the total differentiation order is $\Delta\left(\hat{g}_{1}^{(1)}\right)=\Delta\left(\hat{g}_{1}^{(0)}\right)=2$, Theorem 1.3.4 shows that equation (2.3.77) has no nonpower solutions as $x \rightarrow 0$.

As in the case $a \neq c \neq 0$, to the solution $c_{0}=0$ of the defining equation (2.3.5) there corresponds a family of complicated expansions $\mathcal{B}_{3}$ from (2.3.30).

Solve equation (2.3.1) for $a \neq 0, c=0$ explicitly. To do this, consider equation (2.3.33). It is integrated differently depending on the constant $C_{2}$ and the parameter $a$. Consider three cases of the value of $C_{2}$.

Case $C_{2}=0$. In this case equation (2.3.33) takes the form

$$
\frac{d y}{d \xi}= \pm \sqrt{2 a} y \sqrt{y(y-1)}
$$

It has a solution

$$
y=-\frac{2}{a\left(\xi+C_{3}\right)^{2}-2},
$$

which coincides with (2.3.36) for $c=0$. As $\xi \rightarrow \infty$, (2.3.79) yields the following Laurent expansion in powers of $\xi$ :

$$
y=-\frac{2}{a} \frac{1}{\xi^{2}} \sum_{k=0}^{\infty}(-1)^{k}\left(\frac{C_{4}}{\xi}+\frac{C_{5}}{\xi^{2}}\right)^{k},
$$

where $C_{4} \stackrel{\text { def }}{=}-2 C_{3}$ is an arbitrary constant and $C_{5} \stackrel{\text { def }}{=}-C_{3}-2$.

Taking into account that $\xi=\ln x$, we obtain the explicit form (2.3.79) of expansion (2.3.28).

Case $C_{2}=-2 a$. In this case equation (2.3.33) takes the form

$$
\frac{d y}{d \xi}= \pm \sqrt{2 a} y(y-1)
$$


The integral of this equation is

$$
\frac{y-1}{y}=\exp \left( \pm \sqrt{2 a} \xi+C_{6}\right)
$$

where $C_{6}$ is an arbitrary constant.

Taking into account that $\xi=\ln x$, from (2.3.82) we obtain

$$
y=\frac{1}{1-C_{7} x^{\rho}},
$$

where $\rho=\sqrt{2 a}$ and $C_{7}$ is an arbitrary constant.

For $\operatorname{Re} \rho \geq 0, \rho \neq 0$ and $x \rightarrow 0$ the function (2.3.83) can be expanded in the following series in powers of $C_{8} x^{\rho}$ :

$$
y=\sum_{k=0}^{\infty}(-1)^{k}\left(C_{8} x^{\rho}\right)^{k}
$$

where $C_{8}$ is an arbitrary constant. The series (2.3.84) is an explicit form of expansions (2.3.71), and the function (2.3.83) is the sum of these expansions.

Case $C_{2} \neq 0, C_{2} \neq-2 a$. In this case equation (2.3.33) has the solution (2.3.49) coming from the case $a \neq c \neq 0$.

In the case $a \neq 0, c=0$ formula (2.3.50) becomes

$$
y=-\frac{C_{2}}{2 a} \frac{1}{\beta \cos ^{2}\left[\ln \left(C_{11} x\right)^{\gamma}\right]+\alpha \sin ^{2}\left[\ln \left(C_{11} x\right)^{\gamma}\right]},
$$

where $\gamma=i \sqrt{-C_{2}}, C_{2}$ is an arbitrary positive number, and $\alpha$ and $\beta$ are as in (2.3.43), i.e., $\alpha=1, \beta=-\frac{C_{2}}{2 a}$.

\subsection{Summary of results and discussion.}

Theorem 2.3.1. There are six families of expansions of types 1-3 corresponding to the edge $\Gamma_{4}^{(1)}$ :

$\mathcal{B}_{1}$, which exists for $0 \neq a \neq c \neq 0$, is given by formulas (2.3.18), (2.3.19) and has 1 parameter;

$\mathcal{B}_{2}$, which exists for $a \neq 0, c \neq 0$, is given by formulas (2.3.18), (2.3.19) and has 1 parameter;

$\mathcal{B}_{3}$, which exists for $0 \neq a \neq c$, is given by formulas (2.3.30), (2.3.28), (2.3.36) and has 1 parameter;

$\mathcal{B}_{4}$ and $\mathcal{B}_{5}$, which exist for $a=c \neq 0$, are given by formulas (2.3.58), (2.3.57), (2.3.62) and have 1 parameter;

$\mathcal{B}_{6}$, which exists for $c=0, \rho= \pm \sqrt{2 a}$, $\operatorname{Re} \rho>0$, is given by formulas (2.3.71), (2.3.69) and has 1 parameter;

and four pairs of exotic expansions of type 5 with $\tau= \pm 1$ :

$\mathcal{B}_{0}^{\tau}$, which exist for $a \neq 0$, are given by formula (2.3.4) with first approximation (2.3.2) of the form (2.3.50) and have 2 parameters; the families of inverse expansions $y^{-1}$ for $\mathcal{B}_{0}^{+}$and $\mathcal{B}_{0}^{-}$coincide;

$\mathcal{B}_{1}^{\tau}$, which exists $a \neq c \neq 0, \operatorname{Re}(\sqrt{2 c}-\sqrt{2 a}=0)$, are given by formulas (2.3.14), (2.3.13) with first approximation (2.3.40) and have 1 parameter;

$\mathcal{B}_{2}^{\tau}$, which exist for $a \neq 0, c \neq 0, \operatorname{Re}(\sqrt{2 c}+\sqrt{2 a})=0$, are given by formulas (2.3.14), (2.3.13) with first approximaton (2.3.40) and have 1 parameter;

$\mathcal{B}_{6}^{\tau}$, which exist for $a \neq 0, c=0, \operatorname{Re} \sqrt{2 a}=0$, are given by formulas (2.3.71), (2.3.69) with first approximation (2.3.83) and have 1 parameter. 
The families $\mathcal{B}_{3}, \mathcal{B}_{4}, \mathcal{B}_{5}$ are complicated; $\mathcal{B}_{1}, \mathcal{B}_{2}, \mathcal{B}_{6}$ are power or power-logarithmic.

Remark 2.3.2. The families $\mathcal{B}_{j}^{+}$and $\mathcal{B}_{j}^{-}$have the property that complex exponents in their expansions are complex conjugate. If an expansion in the family $\mathcal{B}_{j}^{+}$coincides with the complex conjugate of an expansion in $\mathcal{B}_{j}^{-}$, then to these two equations there corresponds the same real function. Therefore, it may happen that to an expansion in $\mathcal{B}_{j}^{+}$and an expansion in $\mathcal{B}_{j}^{-}$there corresponds the same function. Usually, this happens when all parameters $a, b, c, d$ in equation (2.1.1) are real. Since we do not assume that all these parameters are real, we view $\mathcal{B}_{j}^{+}$and $\mathcal{B}_{j}^{-}$as different families.

Remark 2.3.3. The height of the edge $\Gamma_{4}^{(1)}$ equals three. The defining equation (2.3.5) has degree three. It turns out that in all cases to each root of this equation there corresponds a family of expansions (or a pair in the case of exotic families).

Remark 2.3.4. Families $\mathcal{A}_{0}, \mathcal{B}_{1}-\mathcal{B}_{6}, \mathcal{B}_{0}^{\tau}, \mathcal{B}_{1}^{\tau}, \mathcal{B}_{2}^{\tau}, \mathcal{B}_{6}^{\tau}$ are called basic families. Other families of expansions are obtained from basic ones using symmetries (2.1.6), (2.1.8) and (2.1.9) of equation $\mathrm{P} 6$.

The existence of families $\mathcal{B}_{1}-\mathcal{B}_{6}, \mathcal{B}_{0}^{\tau}, \mathcal{B}_{1}^{\tau}, \mathcal{B}_{2}^{\tau}, \mathcal{B}_{6}^{\tau}$ for various values of the parameters is shown in the first three columns of Table 1 on page 9 . The basic family $\mathcal{A}_{0}$ exists for all values of the parameters $a, b, c, d$ of equation (2.1.1).

\section{Expansions NeAr Zero CORRESPonding to the EdGe $\Gamma_{1}^{(1)}$}

Families of expansions $\mathcal{H}_{i}, \mathcal{H}_{i}^{\tau}$ corresponding to the edge $\Gamma_{1}^{(1)}$ are obtained from families of expansions $\mathcal{B}_{i}, \mathcal{B}_{i}^{\tau}$ corresponding to the edge $\Gamma_{4}^{(1)}$ using the symmetry (2.1.8). To do this, we need to make the substitution (2.1.8) on the expansion corresponding to the edge $\Gamma_{4}^{(1)}$, to compute expansions with a checkmark corresponding to the edge $\Gamma_{1}^{(1)}$, and to delete the checkmarks. The three cases $a \neq c \neq 0, a=c \neq 0$ and $a \neq 0, c=0$ that we have considered for the edge $\Gamma_{4}^{(1)}$ become the cases $b \neq d-\frac{1}{2} \neq 0, b=d-\frac{1}{2} \neq 0$, and $b \neq 0, d=\frac{1}{2}$ for the edge $\Gamma_{1}^{(1)}$.

4.1. Expansions of solutions for $b \neq 0$. Consider the expansions $\mathcal{B}_{0}^{\tau}$, defined by formula (2.3.4) and corresponding to the edge $\Gamma_{4}^{(1)}$. Using the symmetry (2.1.8) we send them to the expansions $\mathcal{H}_{0}^{\tau}$ corresponding to the edge $\Gamma_{1}^{(1)}$.

Making the substitution $x=\check{x}, y=\check{x} / \check{y}$ in formula (2.3.4) and expressing $\check{y}$ we obtain the fraction

$$
\check{y}=\frac{\check{x}}{c_{\rho} \check{x}^{\rho}+\sum_{s} c_{s} \check{x}^{s}} .
$$

As $\check{x} \rightarrow 0$ the expression (2.4.1) can be expanded in a formal series

$$
\check{y}=\frac{\check{x}^{1-\rho}}{c_{\rho}} \sum_{n=0}^{\infty}\left(-\sum_{s} \frac{c_{s}}{c_{\rho}} \check{x}^{s-\rho}\right)^{n} .
$$

Writing out the first two terms of the series (2.4.2) we obtain the expansion

$$
\check{y}=\frac{\check{x}^{1-\rho}}{c_{\rho}}-\sum_{s} \frac{c_{s}}{c_{\rho}^{2}} \check{x}^{s+1}+\cdots .
$$

Set

$$
\check{\rho}=1-\rho, \quad \check{c}_{\check{\rho}}=\frac{1}{c_{\rho}}, \quad \check{c}_{\check{s}}=-\frac{c_{s}}{c_{\rho}^{2}}, \quad \check{s}=s+1 .
$$


Since $\check{y}=x y^{-1}(x)$ and, according to Theorems 1.5.3 and 2.3.1, the families of inverse expansions $y^{-1}(x)$ for families $\mathcal{B}_{0}^{+}$and $\mathcal{B}_{0}^{-}$coincide, we see that the families $\mathcal{H}_{0}^{+}$and $\mathcal{H}_{0}^{-}$ coincide, forming a single family $\mathcal{H}_{0}$. Therefore we obtain one two-parameter family of semiexotic expansions

$$
\mathcal{H}_{0}: \quad \check{y}=\check{c}_{\check{\rho}} \check{x}^{\check{\rho}}+\check{c}_{1} \check{x}+\check{c}_{2-\check{\rho}} \check{x}^{2-\check{\rho}}+\sum_{\check{s}} \check{c}_{\breve{s}} \check{x}^{\check{s}}+\cdots,
$$

where $\check{\rho}-1$ is an arbitrary purely imaginary constant, $\check{s}$ runs over the set $\{\check{\rho}+l \check{\rho}+$ $m(1-\check{\rho}) ; l, m \in \mathbb{Z} ; l \geq 1 ; m \geq 0\} \cap\{2-\check{\rho}+l(2-\check{\rho})+m(\check{\rho}-1) ; l, m \in \mathbb{Z} ; l \geq 1 ; m \geq 0\}$ $=\{l+k(\check{\rho}-1) ; l, k \in \mathbb{Z} ; l \geq 2 ;|k| \leq l\}$ (according to (2.3.3), (2.3.48), (2.4.4), and Theorem 1.5.2), and the complex coefficients are as follows: $\check{c}_{\check{\rho}}$ is an arbitrary constant, and the other coefficients $\check{c}_{1}, \check{c}_{2-}$, and $\check{c}_{\breve{s}}$ are uniquely determined constants.

4.2. Expansions of solutions for $b \neq d-\frac{1}{2} \neq 0$. Consider the expansions $\mathcal{B}_{i}, \mathcal{B}_{i}^{\tau}$, $i=1,2$, corresponding to the edge $\Gamma_{4}^{(1)}$. Using the symmetry (2.1.8), we transform these expansions to expansions $\mathcal{H}_{i}, \mathcal{H}_{i}^{\tau}, i=1,2$, corresponding to the edge $\Gamma_{1}^{(1)}$.

Set $\check{\theta}_{i}=\sqrt{1-2 d}+(-1)^{i} \sqrt{-2 b}, i=1,2$. For each $i$ one of three cases is possible.

Case $1 . \operatorname{Re} \check{\theta}_{i}=0$. In this case the expansions $\mathcal{B}_{i}, i=1,2$, are given by formula (2.3.14). Substituting $x=\check{x}, y=\frac{\check{x}}{\check{y}}$ and expressing $\check{y}$ we obtain the fraction

$$
\check{y}=\frac{\check{x}}{c_{0 i}+\sum_{s} c_{s i} \check{x}^{s}} .
$$

As $\check{x} \rightarrow 0$ expression (2.4.6) can be expanded in the formal series

$$
\check{y}=\frac{\check{x}}{c_{0 i}} \sum_{n=0}^{\infty}\left(-\sum_{s} \frac{c_{s i}}{c_{0 i}} \check{x}^{s}\right)^{n} .
$$

Writing out the first two cases of the series (2.4.7) we obtain the expansion

$$
\check{y}=\frac{\check{x}}{c_{0 i}}-\sum_{s} \frac{c_{s i}}{c_{0 i}^{2}} \check{x}^{s+1}+\cdots \text {. }
$$

Set

$$
\check{c}_{1 i}=\frac{1}{c_{0 i}}, \quad \check{c}_{\check{s} i}=-\frac{c_{s i}}{c_{0 i}^{2}}, \quad \check{s}=s+1 .
$$

Therefore, for each value of $i$ we obtain two families of exotic expansions

$$
\mathcal{H}_{i}^{\tau}: \quad \check{y}=\check{c}_{1 i} \check{x}+\sum_{\check{s}} \check{c}_{\check{s} i} \check{x}^{\check{s}}+\cdots, \quad i=1,2, \quad \tau= \pm 1,
$$

where, according to (2.3.13) and (2.4.9), $\check{s}$ runs over the set $\left\{1+l+m \tau \check{\theta}_{i}, l, m \in \mathbb{Z}, l, m \geq\right.$ $0, l+m>0\}$, and the complex coefficients are as follows: $\check{c}_{1 i}$ is obtained from (2.1.8), (2.3.6) and (2.4.9) by the formula

$$
\check{c}_{1 i}=\frac{2 b+(-1)^{i} \sqrt{4 b d-2 b}}{2 b-2 d+1},
$$

$\check{c}_{\check{s} i}$ with $\check{s}=1+\tau \check{\theta}_{i}$ is an arbitrary constant, and the other coefficients $\check{c}_{\check{s} i}$ are constant and uniquely defined.

In the case $\check{c}_{\breve{s} i}=0$ with $\check{s}=1+\tau \check{\theta}_{i}$ the second approximation to the expansion of solutions (2.4.10) is $\check{y}=\check{c}_{1 i} \check{x}+\check{c}_{2 i} \check{x}^{2}$. According to (2.1.8) and (2.3.16) we have

$$
\check{c}_{2 i}=(-1)^{i} \sqrt{\frac{1-2 d}{-2 b}} \frac{\left(\sqrt{-2 b}+(-1)^{i} \sqrt{1-2 d}\right)^{2}-2 a+2 c-1}{2-2\left(\sqrt{-2 b}+(-1)^{i} \sqrt{1-2 d}\right)^{2}} .
$$


Expansions (2.4.10) are exotic if $\check{c}_{\check{s} i}$ with $\check{s}=1+\tau \check{\theta}_{i}$ is an arbitrary nonzero constant.

In the case $\check{c}_{\breve{s} i}=0$, where $\check{s}=1+\tau \check{\theta}_{i}$, expansions (2.4.10) are expansions in integral powers of $x$. Families of such expansions are denoted by $\mathcal{H}_{i}, i=1,2$.

Set $k_{i}=1+\check{\theta}_{i}$ if $\operatorname{Re} \check{\theta}_{i}>0$, and $k_{i}=1-\check{\theta}_{i}$ if $\operatorname{Re} \check{\theta}_{i}<0$.

Case 2. $\operatorname{Re} \check{\theta}_{i} \neq 0, \check{\theta}_{i} \notin \mathbb{Z}$. Similarly to the above, using (2.1.8) and (2.4.9), from (2.3.18) we obtain the following one-parameter family of power expansions:

$$
\mathcal{H}_{i}: \quad \check{y}=\check{c}_{1 i} \check{x}+\sum_{\check{s}} \check{c}_{\check{s} i} \check{x}^{\check{s}}+\cdots, \quad i=1,2,
$$

where $\check{s} \in\left\{1+l+m k_{i}, l, m \in \mathbb{Z}, l, m \geq 0, l+m>0\right\}$ and the complex coefficients are as follows: $\check{c}_{1 i}$ is determined by formula (2.4.11), $\check{c}_{k_{i} i}$ is an arbitrary constant, and the remaining $\check{c}_{\breve{s} i}$ are uniquely determined constants.

The second approximation to the expansion of solutions of (2.4.13) depends on Re $k_{i}$. For $\operatorname{Re} k_{i}>2$, the second approximation has the form $\check{y}=\check{c}_{1 i} \check{x}+\check{c}_{2 i} \check{x}^{2}$, which is similar to the case $\operatorname{Re} \check{\theta}_{i}=0$. For $1<\operatorname{Re} k_{i}<2$, the second approximation to solutions takes the form $\check{y}=\check{c}_{1 i} \check{x}+\check{c}_{k_{i} i} \check{x}^{k_{i}}$, where $\check{c}_{k_{i} i}$ is an arbitrary coefficient. For Re $k_{i}=2$, the second approximation of solutions takes the form $\check{y}=\check{c}_{1 i} \check{x}+\check{c}_{2 i} \check{x}^{2}+\check{c}_{k_{i} i} \check{x}^{k_{i}}$, where the coefficients are as follows: $\check{c}_{k_{i} i}$ is arbitrary and $\check{c}_{2 i}$ is given by formula (2.4.12).

Case 3. $\check{\theta}_{i} \in \mathbb{Z} \backslash\{0\}$. We have a one-parameter family of power-logarithmic expansions

$$
\mathcal{H}_{i}: \quad \check{y}=\check{c}_{1 i} \check{x}+\sum_{\breve{s}=2}^{\infty} \check{c}_{\check{s} i}(\ln \check{x}) \check{x}^{\check{s}}+\cdots, \quad i=1,2,
$$

where the coefficient $\check{c}_{1 i}$ is given by formula (2.4.11), $\check{c}_{k_{i} i}=\check{\alpha}_{k_{i} i}+\check{\beta}_{k_{i} i} \ln \check{x}, \check{\alpha}_{k_{i} i}$ is an arbitrary constant, $\check{\beta}_{k_{i} i}$ is a uniquely determined constant, and the remaining $\check{c}_{\check{s} i}$ are uniquely determined polynomials in $\ln \check{x}$.

The second approximation to the expansion of solution (2.4.14) depends on the value of $k_{i}$. If $k_{i}=2$, then the second approximation takes the form $y=\check{c}_{1 i} \check{x}+\check{c}_{2 i} \check{x}^{2}$. According to (2.1.8) and (2.3.20), we have

$$
\check{c}_{2 i}=\check{\alpha}_{2 i}+(-1)^{i} \sqrt{\frac{1-2 d}{-2 b}} \frac{\left(\sqrt{-2 b}+(-1)^{i} \sqrt{1-2 d}\right)^{2}-2 a+2 c-1}{4} \ln \check{x},
$$

where $\check{\alpha_{2 i}}$ is an arbitrary constant.

If $\left(\sqrt{-2 b}+(-1)^{i} \sqrt{1-2 d}\right)^{2}-2 a+2 c-1=0$, then the compatibility condition is satisfied and expansion (2.4.14) does not contain logarithms.

Similarly, using symmetry (2.1.8) we obtain from (2.3.30) the following one-parameter system of complicated expansions:

$$
\mathcal{H}_{3}: \quad \check{y}=\check{\varphi}_{1} \check{x}+\sum_{\check{\sigma}=2}^{\infty} \check{\varphi}_{\check{\sigma}} \check{x} \check{\sigma}
$$

where

$$
\check{\varphi}_{1}=\frac{1+2 b-2 d}{4} \ln ^{2} \check{x}+\check{c}_{1} \ln \check{x}+\sum_{\check{s}_{=0}}^{\infty} \check{c}_{-\check{s}} \ln ^{-\check{s}} \check{x}
$$

and the complex coefficients are as follows: $\check{c}_{1}$ is arbitrary, the remaining $\check{c}_{-\breve{s}}$ are uniquely determined constants, and the $\check{\varphi}_{\check{\sigma}}$ are complex series in decreasing powers of logarithms. 
4.3. Expansion of solutions for $b=d-\frac{1}{2} \neq 0$. Expansions (2.3.58) yield two oneparameter families of complicated expansions

$$
\mathcal{H}_{3+j}: \quad \check{y}=\check{\phi}_{1 j} \check{x}+\sum_{\check{\sigma}=2}^{\infty} \check{\phi}_{\check{\sigma} j} \check{x}^{\check{\sigma}}, \quad j=1,2,
$$

where

$$
\check{\phi}_{1 j}=(-1)^{j} \sqrt{-2 b} \ln \check{x}+\check{c}_{0 j}+\sum_{\check{s}=1}^{\infty} \check{c}_{-\check{s} j} \ln ^{-\check{s}} \check{x}, \quad j=1,2,
$$

and the complex coefficients are as follows: $\check{c}_{0 j}$ is an arbitrary constant, the remaining $\check{c}_{-\check{s} j}$ are uniquely determined constants, and the $\phi_{\breve{\sigma} j}$ are series in decreasing powers of logarithms.

The families of expansions $\mathcal{H}_{2}$ and $\mathcal{H}_{2}^{\tau}$ are coming from the case $b \neq d-\frac{1}{2} \neq 0$. For the value of $\check{\theta}_{2}$ we take $2 \sqrt{-2 b}$ with $\operatorname{Re} \check{\theta}_{2} \geq 0$. Depending on this value, the following three cases are possible: Case $1\left(\operatorname{Re} \check{\theta}_{2}=0\right.$, families of exotic expansions $\mathcal{H}_{2}^{\tau}$ are given by formula $(2.4 .10)$, Case $2\left(\operatorname{Re} \check{\theta}_{2} \neq 0, \check{\theta}_{2} \notin \mathbb{Z}\right.$, one-parameter family of power expansions $\mathcal{H}_{2}$ is given by formula $(2.4 .13)$ ), Case $3\left(\check{\theta}_{2} \in \mathbb{Z} \backslash\{0\}\right.$, one-parameter family of powerlogarithmic expansions $\mathcal{H}_{2}$ is given by formula (2.4.14) $)$.

4.4. Expansion of solutions for $d=\frac{1}{2}, b \neq 0$. In the case $\operatorname{Re} \sqrt{-2 b}>0$ we have a one-parameter family of power expansions

$$
\mathcal{H}_{6}: \quad \check{y}=\check{x}+\check{c}_{\check{\rho}} \check{x}^{\check{\rho}}+\sum_{\check{s}} \check{c}_{\check{s}} \check{x}^{\check{s}},
$$

where $\check{c}_{\check{\rho}} \neq 0, \check{c}_{\check{\rho}}$ is an arbitrary constant, $\check{\rho}=1+\sqrt{-2 b}$, $\check{s}$ runs over the set $\{\check{\rho}+$ $l(\check{\rho}-1)+m ; l, m \geq 0 ; l+m>0 ; l, m \in \mathbb{Z}\}$, and the remaining complex coefficients $\check{c}_{\breve{s}}$ are uniquely determined constants.

For $\operatorname{Re} \check{\rho}>2$ the third approximation to expansion (2.4.20) is $\check{y}=\check{x}+\check{c}_{\breve{\rho}} \check{x}^{\check{\rho}}+$ $\check{c}_{\breve{\rho}+1} \check{x}^{\check{\rho}+1}$. The coefficient is given by

$$
\check{c}_{\check{\rho}+1}=-\check{c}_{\breve{\rho}} \frac{2 b+2 a-2 c+1}{2 \check{\rho}} .
$$

For $1<\operatorname{Re} \check{\rho}<2$ the third approximation to expansion (2.4.20) is $\check{y}=\check{x}+\check{c}_{\check{\rho}} \check{x}^{\check{\rho}}+$ $\check{c}_{2 \check{\rho}-1} \check{x}^{2 \check{\rho}-1}$. The coefficient is given by

$$
\check{c}_{2 \check{\rho}-1}=-\check{c}_{\check{\rho}}^{2} .
$$

For $\operatorname{Re} \check{r}=2, \operatorname{Im} \rho \neq 0$ the third approximation to expansion (2.4.20) is given by $\check{y}=$ $\check{x}+\check{c}_{\breve{\rho}} \check{x}^{\breve{\rho}}+\check{c}_{\breve{\rho}+1} \check{x}^{\breve{\rho}+1}+\check{c}_{2 \breve{\rho}-1} \check{x}^{2 \check{\rho}-1}$, where the coefficients $\check{c}_{\breve{\rho}+1}$ and $\check{c}_{2 \breve{\rho}-1}$ are defined by formulas (2.4.21) and (2.4.22) respectively. In the case $\check{\rho}=2$ the third approximation to expansions (2.4.20) is $\check{y}=\check{x}+\check{c}_{2} \check{x}^{2}+\check{c}_{3} \check{x}^{3}$. The coefficients are as follows: $\check{c}_{2}$ is an arbitrary constant and $\check{c}_{3}=-\check{c}_{2} \frac{\left(2 b+2 a-2 \check{c}+1+4 \check{c}_{2}\right)}{4}$ is the sum of (2.4.21) and (2.4.22).

In the case $\operatorname{Re} \sqrt{-2 b}=0$ we have two one-parameter families of exotic expansions

$$
\mathcal{H}_{6}^{\tau}: \quad \check{y}=\check{x}+\check{c}_{\check{\rho}} \check{x}^{\check{\rho}}+\sum_{\check{s}} \check{c}_{\check{s}} \check{x}^{\check{s}}, \quad \tau= \pm 1,
$$

where $\check{\rho}=1+\sqrt{-2 b}, \check{s}$ runs over the set $\{\check{\rho}+l(\check{\rho}-1)+m ; l, m \geq 0 ; l+m>0 ; l, m \in \mathbb{Z}\}$, and the complex coefficients are as follows: $\check{c}_{\check{\rho}}$ is an arbitrary nonzero constant and the remaining $\check{c}_{\check{s}}$ are uniquely determined constants.

There is also a one-parameter family $\mathcal{H}_{3}$ given by formulas (2.4.16), (2.4.17). 


\subsection{Summary of results and discussion.}

Theorem 2.4.1. There are 6 families of expansions of types 1-3 corresponding to the edge $\Gamma_{1}^{(1)}$ :

$\mathcal{H}_{1}=\check{\mathcal{B}}_{1}$, which exists for $b \neq d-\frac{1}{2} \neq 0$, is given by formulas (2.4.13), (2.4.14), and has 1 parameter;

$\mathcal{H}_{2}=\check{\mathcal{B}}_{2}$, which exists for $d \neq \frac{1}{2}$, is given by formulas (2.4.13), (2.4.14), and has 1 parameter;

$\mathcal{H}_{3}=\check{\mathcal{B}}_{3}$, which exists for $b \neq d-\frac{1}{2}$, is given by formulas (2.4.16), (2.4.17) and has 1 parameter;

$\mathcal{H}_{4}=\check{\mathcal{B}}_{4}$ and $\mathcal{H}_{5}=\check{\mathcal{B}}_{5}$, which exist for $b=d-\frac{1}{2}$, are given by formulas (2.4.18), (2.4.19), and have 1 parameter;

$\mathcal{H}_{6}=\tilde{\mathcal{B}}_{6}$, which exists for $d=\frac{1}{2}$, is given by formula (2.4.20) and has 1 parameter;

one family of semiexotic expansions:

$\mathcal{H}_{0}=\check{\mathcal{B}}_{0}^{\tau}$, which exists for $b \neq 0$, is given by formula (2.4.5), and has 2 parameters;

and 3 pairs of families of exotic expansions of solutions with $\tau= \pm 1$ :

$\mathcal{H}_{1}^{\tau}=\check{\mathcal{B}}_{1}^{\tau}$, which exist for $b \neq d-\frac{1}{2} \neq 0, \operatorname{Re}(\sqrt{1-2 d}-\sqrt{-2 b})=0$, are given by formula (2.4.10), and have 1 parameter each;

$\mathcal{H}_{2}^{\tau}=\check{\mathcal{B}}_{2}^{\tau}$, which exist for $b \neq 0, d \neq \frac{1}{2}, \operatorname{Re}(\sqrt{1-2 d}+\sqrt{-2 b})=0$, are given by formula (2.4.10), and have 1 parameter;

$\mathcal{H}_{6}^{\tau}=\check{\mathcal{B}}_{6}^{\tau}$, which exist for $b \neq 0, d=\frac{1}{2}, \operatorname{Re} \sqrt{-2 b}=0$, are given by formula (2.4.23), and have 1 parameter.

Here $\check{\mathcal{B}}_{i}$ is the family obtained from $\mathcal{B}_{i}$ by applying the symmetry (2.1.8).

Families $\mathcal{H}_{3}, \mathcal{H}_{4}, \mathcal{H}_{5}$ are complicated; families $\mathcal{H}_{1}, \mathcal{H}_{2}, \mathcal{H}_{6}$ are power or powerlogarithmic.

\section{EXPANSIONS NEAR INFINITY}

Starting with expansions of solutions of equation (2.1.1) as $x \rightarrow 0$ we use the symmetry (2.1.6) to obtain expansions of solutions of this equation as $x \rightarrow \infty$. To do this, in expansions as $x \rightarrow 0$ we make the substitution (2.1.6), compute expansions with asterisks as $x \rightarrow \infty$ and then remove the asterisks.

Let us consider in detail how to transform the expansion corresponding to the vertex $\Gamma_{4}^{(0)}$ to the expansion corresponding to the vertex $\Gamma_{2}^{(0)}$. The remaining expansions near infinity, which correspond to the edges $\Gamma_{2}^{(1)}$ and $\Gamma_{3}^{(1)}$, will be just listed.

5.1. Expansions corresponding to the vertex $\Gamma_{2}^{(0)}$. These expansions will be obtained from expansions in the family $\mathcal{A}_{0}$ corresponding to the vertex $\Gamma_{4}^{(0)}$ and defined by formula (2.2.6), where the exponent $r$ is any number satisfying the condition $0<\operatorname{Re} r<1, s$ runs over the set (2.2.5), and the complex coefficients are given as follows: $c_{r}$ is an arbitrary constant and the remaining $c_{s}$ are uniquely determined constants. Substituting in (2.1.2) $x=\frac{1}{x^{*}}, y=\frac{1}{y^{*}}$ and expressing $y^{*}$ we obtain the fraction

$$
y^{*}=\frac{1}{c_{r} x^{*-r}+\sum_{s} c_{s} x^{*-s}},
$$

where $c_{r} \neq 0$ is an arbitrary constant, the remaining coefficients $c_{s}$ are uniquely determined constants, the exponent $r$ is arbitrary with $0<\operatorname{Re} r<1$, and $s$ runs over the set 
(2.2.5). As $x^{*} \rightarrow \infty,(2.5 .1)$ can be expanded in a formal series

$$
y^{*}=\frac{x^{* r}}{c_{r}} \sum_{n=0}^{\infty}\left(-\sum_{s} \frac{c_{s}}{c_{r}} x^{*-s+r}\right)^{n} .
$$

Taking the first two terms of series (2.5.2) we obtain the expansion

$$
y^{*}=\frac{1}{c_{r}} x^{* r}-\sum_{s}^{\infty} \frac{c_{s}}{c_{r}^{2}} x^{*-s+2 r}+\cdots \text {. }
$$

Set

$$
c_{r}^{*}=\frac{1}{c_{r}}, \quad c_{s^{*}}^{*}=-\frac{c_{s}}{c_{r}^{2}}, \quad s^{*}=-s+2 r .
$$

Finally, taking into account (2.2.5) and (2.5.4) we obtain the expansion

$$
y^{*}=c_{r}^{*} x^{* r}+\sum_{s^{*}} c_{s^{*}}^{*} x^{* s^{*}}
$$

where the exponent $r$ is arbitrary with $0<\operatorname{Re} r<1, s^{*} \in\{r-l r+m(r-1)$; $l, m \geq 0 ; l+m>0 ; l, m \in \mathbb{Z}\}$, and the complex coefficients are as follows: $c_{r}^{*}$ is an arbitrary nonzero constant, and the remaining $c_{s^{*}}^{*}$ are uniquely determined constants.

Therefore, as $x^{*} \rightarrow \infty$, we have a two-parameter (with parameters $c_{r}^{*}$ and $r$ ) family of expansions $\mathcal{A}_{\infty}$ defined by formula (2.5.5).

Let us compute the second approximation to the expansion. We consider four cases.

In case $\frac{1}{2}<\operatorname{Re} r<1$ the second approximation to the solution is $y^{*}=c_{r}^{*} x^{* r}+$ $c_{2 r-1}^{*} x^{* 2 r-1}$. The coefficient is given by the following formula:

$$
c_{2 r-1}^{*}=c_{r}^{* 2} \frac{2(a+d)+(r-1)^{2}-1}{2(r-1)^{2}} .
$$

In case $0<\operatorname{Re} r<\frac{1}{2}$ the second approximation to the solution is $y^{*}=c_{r}^{*} x^{* r}+c_{0}^{*}$. The coefficient is given by the following formula:

$$
c_{0}^{*}=\frac{2(b+c)-r^{2}}{2 r^{2}} .
$$

In case $r=\frac{1}{2}$ the second approximation to the solution is $y^{*}=c_{\frac{1}{2}}^{*} \sqrt{x^{*}}+c_{0}^{*}$, where $c_{\frac{1}{2}}^{*}$ is an arbitrary constant and the coefficient is given by the formula

$$
c_{0}^{*}=\frac{\left(-1+8(b+c)-3 c_{\frac{1}{2}}^{* 2}+8 c_{\frac{1}{2}}^{* 2}(a+d)\right)}{2} .
$$

In case $\operatorname{Re} r=\frac{1}{2}, \operatorname{Im} r \neq 0$ the second approximation to the solution is $y^{*}=c_{r}^{*} x^{* r}+$ $c_{0}^{*}+c_{2 r-1}^{*} x^{* 2 r-1}$, where the coefficients $c_{2 r-1}^{*}$ and $c_{0}^{*}$ are defined by formulas (2.5.6) and (2.5.7) respectively.

5.2. Expansions of solutions corresponding to the edge $\Gamma_{2}^{(1)}$. Here we consider four cases: $b \neq 0,-b \neq c \neq 0,-b=c \neq 0$ and $b \neq 0, c=0$.

Expansions of solutions for $b \neq 0$. We have one two-parameter family of exotic expansions

$$
\mathcal{D}_{0}: \quad y^{*}=c_{\rho^{*}}^{*} x^{* \rho^{*}}+c_{0}^{*}+c_{-\rho^{*}}^{*} x^{*-\rho^{*}}+\sum_{s^{*}} c_{s^{*}}^{*} x^{* s^{*}},
$$

where $\rho^{*} \neq 0$ is an arbitrary purely imaginary constant, $s^{*}$ runs over the set $\left\{l+k \rho^{*} ; l \leq\right.$ $-1 ;|k| \leq|l|+1 ; l, k \in \mathbb{Z}\}$ and the complex coefficients are as follows: $c_{\rho^{*}}^{*}$ is an arbitrary nonzero constant, and $c_{0}^{*}, c_{-\rho^{*}}^{*}$, and $c_{s^{*}}^{*}$ are uniquely determined constants. 
Expansions of solutions for $-b \neq c \neq 0$. We set $\theta_{i}^{*} \stackrel{\text { def }}{=} \sqrt{2 c}+(-1)^{i} \sqrt{-2 b}, i=1,2$. For a fixed $i$ there are three possible cases depending on the value of $\theta_{i}^{*}$.

Case $1 . \operatorname{Re} \theta_{i}^{*}=0$. We have two one-parameter families of semiexotic expansions

$$
\mathcal{D}_{i}^{\tau}: \quad y^{*}=c_{0 i}^{*}+\sum_{s^{*}} c_{s^{*} i} x^{* s}, \quad \tau= \pm 1
$$

where $s^{*} \in\left\{-l+m \tau \theta_{i}^{*} ; l, m \geq 0 ; l+m>0 ; l, m \in \mathbb{Z}\right\}$ and the complex coefficients are given as follows: $c_{s^{*} i}^{*}$ with $s^{*}=\tau \theta_{i}^{*}$ is an arbitrary constant, and $c_{0 i}^{*}$ is obtained from (2.1.6), (2.3.6), and (2.5.4) by the formula

$$
c_{0 i}^{*}=\frac{b+(-1)^{i} \sqrt{-b c}}{b+c}, \quad i=1,2 .
$$

The remaining $c_{s^{*} i}^{*}$ are uniquely determined constants.

If $c_{s^{*} i}^{*}=0$ with $s^{*}=\tau \theta_{i}^{*}$, then the second approximation to the expansions of solutions (2.5.9) is $y^{*}=c_{0 i}^{*}+c_{-1 i}^{*} x^{*-1}$. Formulas (2.1.6), (2.3.16), and (2.5.4) imply that the coefficient is given by the following formula:

$$
c_{-1 i}^{*}=(-1)^{i} \sqrt{-\frac{c}{b}} \frac{\left(\sqrt{-b}+(-1)^{i} \sqrt{c}\right)^{2}-a-d}{1-2\left(\sqrt{-b}+(-1)^{i} \sqrt{c}\right)^{2}} .
$$

In the case $c_{s^{*} i}^{*}=0$ with $s^{*}=\tau \theta_{i}^{*}$ expansions (2.5.9) are expansions in integral powers of $x$. Families of such expansions are denoted by $\mathcal{D}_{i}, i=1,2$.

Let $k_{i}=\theta_{i}^{*}$ if $\operatorname{Re} \theta_{i}^{*}<0$ and $k_{i}=-\theta_{i}^{*}$ if $\operatorname{Re} \theta_{i}^{*}>0$.

Case $2 . \operatorname{Re} \theta_{i}^{*} \neq 0, \theta_{i}^{*} \notin \mathbb{Z}$. We have the one-parameter family of power expansions

$$
\mathcal{D}_{i}: \quad y^{*}=c_{0 i}^{*}+\sum_{s^{*}} c_{s^{*} i}^{*} x^{* s^{*}}, \quad i=1,2,
$$

where $s^{*} \in\left\{-l+m k_{i} ; l, m \in \mathbb{Z} ; l, m \geq 0 ; l+m>0\right\}$, and the complex coefficients are as follows: $c_{0 i}^{*}$ is defined by formula (2.5.10),,$c_{k_{i} i}^{*}$ is arbitrary, and the remaining $c_{s^{*} i}^{*}$ are arbitrary constants. The second approximation to expansions of solutions (2.5.12) depends on the number $\operatorname{Re} k_{i}$. If $\operatorname{Re} k_{i}<-1$, then the second approximation of solutions has the form $y^{*}=c_{0 i}^{*}+c_{-1 i}^{*} x^{*-1}$, where the coefficient $c_{-1 i}^{*}$ is given by formula (2.5.11). If $-1<\operatorname{Re} k_{i}<0$, then the second approximation is of the form $y^{*}=c_{0 i}^{*}+c_{k_{i}}^{*} x^{* k_{i}}$, where the coefficient $c_{k_{i}}^{*}$ is arbitrary. If $\operatorname{Re} k_{i}=-1$, then the second approximation is of the form $y^{*}=c_{0 i}^{*}+c_{-1 i}^{*} x^{*-1}+c_{k_{i} i}^{*} x^{* k_{i}}$, where the coefficients are as follows: $c_{k_{i} i}^{*}$ is arbitrary and $c_{-1 i}^{*}$ is given by formula (2.5.11).

Case 3. $\theta_{i}^{*} \in \mathbb{Z} \backslash\{0\}$. We have a one-parameter family of power-logarithmic expansions

$$
\mathcal{D}_{i}: \quad y^{*}=c_{0 i}^{*}+\sum_{s^{*}=1}^{+\infty} c_{-s^{*} i}\left(\ln x^{*}\right) x^{*-s^{*}}, \quad i=1,2,
$$

where the coefficients are as follows: $c_{0 i}^{*}$ is given by formula (2.3.6); $; c_{k_{i} i}^{*}=\alpha_{k_{i} i}^{*}+\beta_{k_{i} i}^{*} \ln x^{*}$ with an arbitrary constant $\alpha_{k_{i} i}^{*}$, the coefficient $\beta_{k_{i} i}^{*}$ is constant and uniquely determined, and the complex coefficients $c_{s^{*} i}^{*}$ are uniquely determined polynomials in $\ln x^{*}$. The second approximation to the expansion of solution (2.5.13) depends on the value of $k_{i}$. If $k_{i}=-1$, then the second approximation takes the form $y^{*}=c_{0 i}^{*}+c_{-1 i}^{*} x^{*-1}$. The coefficient is given by the following formula:

$$
c_{-1 i}^{*}=\alpha_{-1 i}^{*}+(-1)^{i} \sqrt{-\frac{c}{b}} \frac{\left(\sqrt{-b}+(-1)^{i} \sqrt{c}\right)^{2}-a-d}{2} \ln x^{*},
$$

where $\alpha_{-1 i}^{*}$ is an arbitrary constant. 
We also have a one-parameter family of complicated expansions

$$
\mathcal{D}_{3}: \quad y^{*}=\varphi_{0}^{*}+\sum_{\sigma^{*}=1}^{\infty} \varphi_{-\sigma^{*}}^{*} x^{-* \sigma^{*}}
$$

where

$$
\varphi_{0}^{*}=\frac{b+c}{2} \ln ^{2} x^{*}+c_{1}^{*} \ln x^{*}+\sum_{s^{*}=0}^{\infty} c_{-s^{*}}^{*} \ln ^{-s^{*}} x^{*},
$$

and the complex coefficients are as follows: $c_{1}^{*}$ is an arbitrary constant, $c_{-s^{*}}^{*}$ are uniquely determined constants, and $\varphi_{-\sigma^{*}}^{*}$ are series in decreasing powers of $\ln x^{*}$.

Expansions of solutions for $-b=c \neq 0$. In this case there exist two one-parameter families of complicated expansions

$$
\mathcal{D}_{3+j}: \quad y^{*}=\phi_{0 j}^{*}+\sum_{\sigma^{*}=1}^{\infty} \phi_{-\sigma^{*} j}^{*} x^{*-\sigma^{*}}, \quad j=1,2,
$$

where

$$
\phi_{0 j}^{*}=(-1)^{j} \sqrt{-2 b} \ln x^{*}+c_{0 j}^{*}+\sum_{s^{*}=1}^{\infty} c_{-s^{*} j} \ln ^{-s^{*}} x^{*}, \quad j=1,2,
$$

and the complex coefficients are as follows: $c_{0 j}^{*}$ is an arbitrary constant, $c_{-s^{*} j}^{*}$ are uniquely determined constants, and $\phi_{-\sigma^{*} j}^{*}$ are series in decreasing powers of $\ln x^{*}$.

The families of expansions $\mathcal{D}_{2}$ and $\mathcal{D}_{2}^{\tau}$ described for the case $-b \neq c \neq 0$ remain in the present case as well. For $\theta_{2}^{*}$ we take $2 \sqrt{-2 b}$ with $\operatorname{Re} \sqrt{-2 b} \leq 0$. Depending on the value of $\theta_{2}^{*}$ three cases are possible, namely, Case $1\left(\operatorname{Re} \theta_{2}^{*}=0\right.$, two one-parameter families of exotic expansions $\mathcal{D}_{2}^{\tau}$ are given by formula (2.5.9) $)$, Case $2\left(\operatorname{Re} \theta_{2}^{*} \neq 0, \theta_{2}^{*} \notin \mathbb{Z}\right.$, one-parameter family of power expansions $\mathcal{D}_{2}$ is determined by formula (2.5.12) ), Case 3 $\left(\theta_{2}^{*} \notin \mathbb{Z}\right.$, a one-parameter of power-logarithmic expansions $\mathcal{D}_{2}$ is determined by formula $(\underline{2.5 .13})$ ).

Expansion of solutions for $b \neq 0, c=0$. For $\operatorname{Re} \sqrt{-2 b}<0$ we have a one-parameter family of power expansions

$$
\mathcal{D}_{6}: \quad y^{*}=1+c_{\rho^{*}}^{*} x^{* \rho^{*}}+\sum_{s^{*}} c_{s^{*}}^{*} x^{* s^{*}},
$$

where $\rho^{*}=\sqrt{-2 b}, s^{*}$ runs over the set $\left\{\rho^{*}+l \rho^{*}-m ; l, m \geq 0 ; l+m>0 ; l, m \in \mathbb{Z}\right\}$, and the complex coefficients are as follows: $c_{\rho^{*}}^{*}$ is an arbitrary nonzero constant, and the remaining $c_{s^{*}}^{*}$ are uniquely determined constants.

If $\operatorname{Re} \rho^{*}<-1$, then the third approximation to expansion (2.5.19) is $y=1+c_{\rho^{*}}^{*} x^{* \rho^{*}}+$ $c_{\rho^{*}-1}^{*} x^{* \rho^{*}-1}$. The coefficient is given by the formula

$$
c_{\rho^{*}-1}^{*}=c_{\rho}^{*} \frac{a+b+d}{\rho^{*}-1} .
$$

If $-1<\operatorname{Re} \rho^{*}<0$, then the third approximation to expansion (2.5.19) is $y^{*}=1+$ $c_{\rho^{*}}^{*} x^{* \rho^{*}}+c_{2 \rho^{*}}^{*} x^{* 2 \rho^{*}}$. The coefficient is given by the formula

$$
c_{2 \rho^{*}}^{*}=-c_{\rho}^{* 2} .
$$

If $\operatorname{Re} \rho^{*}=-1, \operatorname{Im} \rho^{*} \neq 0$, then the third approximation to expansion (2.5.19) is $y^{*}=$ $1+c_{\rho^{*}}^{*} x^{* \rho^{*}}+c_{\rho^{*}-1}^{*} x^{* \rho^{*}-1}+c_{2 \rho^{*}}^{*} x^{* 2 \rho^{*}}$, where the coefficients $c_{\rho^{*}-1}^{*}$ and $c_{2 \rho^{*}}^{*}$ are given by formulas (2.5.20) and (2.5.21) respectively. If $\rho^{*}=-1$, then the third approximation to expansion (2.5.19) is $y^{*}=1+c_{-1}^{*} x^{*-1}+c_{-2}^{*} x^{*-2}$. The coefficients are as follows: $c_{-1}^{*}$ is 
an arbitrary nonzero constant, and $c_{-2}^{*}=-c_{-1}^{*} \frac{\left(a+b+d+2 c_{-1}^{*}\right)}{2}$ is the sum of (2.5.20) and (2.5.21).

For $\operatorname{Re} \sqrt{-2 b}=0$ there exist two one-parameter families of exotic expansions

$$
\mathcal{D}_{6}^{\tau}: \quad y^{*}=1+c_{\rho^{*}}^{*} x^{* \rho^{*}}+\sum_{s^{*}} c_{s^{*}}^{*} x^{s^{*}},
$$

where $\rho^{*}=\sqrt{-2 b}, s^{*}$ runs over the set $\left\{\rho^{*}+l \rho^{*}-m ; l, m \geq 0 ; l+m>0 ; l, m \in \mathbb{Z}\right\}$, $\tau= \pm 1$, and the complex coefficients are as follows: $c_{\rho^{*}}^{*}$ is an arbitrary nonzero constant, and all $c_{s^{*}}^{*}$ are uniquely determined constants.

Also there exists a one-parameter family of complicated expansions $\mathcal{D}_{3}$ given by formulas (2.5.15), (2.5.16).

5.3. Expansions of solutions corresponding to the edge $\Gamma_{3}^{(1)}$. Here we also consider four cases: $a \neq 0, a \neq-d+1 / 2 \neq 0, a=-d+1 / 2 \neq 0$ and $d=1 / 2, a \neq 0$.

Expansion of solutions for $a \neq 0$. We have two two-parameter families of exotic expansions:

$$
\mathcal{G}_{0}^{\tau}: \quad y^{*}=c_{\rho^{*}}^{*} x^{* \rho^{*}}+\sum_{s^{*}} c_{s^{*}}^{*} x^{* s^{*}}, \quad \tau=\operatorname{sgn} \operatorname{Im}\left(\rho^{*}-1\right),
$$

where $\rho^{*}-1$ is an arbitrary purely imaginary constant, $s^{*}$ runs over the set $\left\{\rho^{*}-l \rho^{*}+\right.$ $\left.m\left(\rho^{*}-1\right) ; l, m \geq 0 ; l+m>0 ; l, m \in \mathbb{Z}\right\}$, and the complex coefficients are as follows: $c_{\rho^{*}}^{*}$ is an arbitrary constant, and $c_{s^{*}}^{*}$ are uniquely determined constants.

Expansions of solutions for $a \neq-d+\frac{1}{2} \neq 0$. Set $\theta_{i}^{*} \stackrel{\text { def }}{=} \sqrt{1-2 d}+(-1)^{i} \sqrt{2 a}, i=1,2$. For each $i$, depending on the value of $\theta_{i}^{*}$ one of the three cases is possible.

Case $1 . \operatorname{Re} \theta_{i}^{*}=0$. We have two one-parameter families of exotic expansions

$$
\mathcal{G}_{i}^{\tau}: \quad y^{*}=c_{1 i}^{*} x^{*}+\sum_{s^{*}} c_{s^{*} i}^{*} x^{* s^{*}}, \quad i=1,2, \quad \tau= \pm 1,
$$

where $s^{*} \in\left\{1-l+m \tau \theta_{i}^{*} ; l, m \geq 0 ; l+m>0 ; l, m \in \mathbb{Z}\right\}$, and the complex coefficients are as follows: $c_{s^{*} i}^{*}$ with $s^{*}=1+\tau \theta_{i}^{*}$ is an arbitrary constant, $c_{1 i}$ is obtained from (2.1.6), (2.4.11) and (2.5.4) by the formula

$$
c_{1 i}^{*}=1+(-1)^{i} \sqrt{\frac{1-2 d}{2 a}},
$$

and the remaining $c_{s^{*} i}^{*}$ are uniquely determined constants.

If $c_{s^{*} i}^{*}=0$ with $s^{*}=1+\tau \theta_{i}^{*}$, then the second approximation to expansions of solutions (2.5.24) is $y^{*}=c_{1 i}^{*} x^{*}+c_{0 i}^{*}$. Formulas (2.1.6), (2.4.12) and (2.5.4) imply that the coefficient $c_{0 i}^{*}$ is given by the formula

$$
c_{0 i}^{*}=(-1)^{i} \sqrt{\frac{1-2 d}{2 a}} \frac{\left(\sqrt{2 a}+(-1)^{i} \sqrt{1-2 d}\right)^{2}+2 b+2 c-1}{2-2\left(\sqrt{2 a}+(-1)^{i} \sqrt{1-2 d}\right)^{2}} .
$$

In the case $c_{s^{*} i}^{*}=0$ with $s^{*}=1+\tau \theta_{i}^{*}$ expansions (2.5.24) are expansions in integral powers of $x$. Families of such expansions are denoted $\mathcal{G}_{i}, i=1,2$.

Set $k_{i}=1+\theta_{i}^{*}$ if $\operatorname{Re} \theta_{i}^{*}<0$ and $k_{i}=1-\theta_{i}^{*}$ if $\operatorname{Re} \theta_{i}^{*}>0$.

Case 2. $\operatorname{Re} \theta_{i}^{*} \neq 0, \theta_{i}^{*} \notin \mathbb{Z}$. Here we have a one-parameter family of power expansions

$$
\mathcal{G}_{i}: \quad y^{*}=c_{1 i}^{*} x^{*}+\sum_{s^{*}} c_{s^{*} i}^{*} x^{* s^{*}}, \quad i=1,2,
$$

where the complex coefficients are as follows: $c_{1 i}^{*}$ is given by formula (2.5.25), $c_{k_{i} i}^{*}$ is arbitrary, and the remaining $c_{s^{*} i}^{*}$ are uniquely determined constants. The parameter $s^{*}$ 
runs over the set $\left\{1-l+m k_{i} ; l, m \in \mathbb{Z} ; l, m \geq 0 ; l+m>0\right\}$. The second approximation to the expansion of solutions (2.5.27) depends on the number $\operatorname{Re} k_{i}$. If $\operatorname{Re} k_{i}<0$, then the second approximation is $y^{*}=c_{1 i}^{*} x^{*}+c_{0 i}^{*}$, where the coefficient $c_{0 i}^{*}$ is given by formula (2.5.26). If $0<\operatorname{Re} k_{i}<1$, then the second approximation is $y^{*}=c_{1 i}^{*} x^{*}+c_{k_{i}}^{*} x^{* k_{i}}$, where $c_{k_{i} i}^{*}$ is an arbitrary coefficient. If $\operatorname{Re} k_{i}=0$, then the second approximation is $y^{*}=c_{1 i}^{*} x^{*}+c_{0 i}^{*}+c_{k_{i} i}^{*} x^{* k_{i}}$, where the coefficients are as follows: $c_{k_{i} i}^{*}$ is arbitrary, and $c_{0 i}^{*}$ is given by formula (2.5.26).

Case 3. $\theta_{i}^{*} \in \mathbb{Z} \backslash\{0\}$. We have one-parameter power-logarithmic expansions

$$
\mathcal{G}_{i}: \quad y^{*}=c_{1 i}^{*} x^{*}+\sum_{s^{*}=0}^{+\infty} c_{s^{*} i}^{*}\left(\ln x^{*}\right) x^{* s^{*}}, \quad i=1,2,
$$

where the coefficients are as follows: $c_{1 i}^{*}$ is given by formula (2.5.25), $c_{k_{i} i}^{*}=\alpha_{k_{i} i}^{*}+$ $\beta_{k_{i} i}^{*} \ln x^{*}, \alpha_{k_{i} i}^{*}$ is an arbitrary constant, $\beta_{k_{i} i}^{*}$ is a uniquely determined constant, and the remaining $c_{s^{*} i}^{*}$ are uniquely determined polynomials in $\ln x^{*}$. The second approximation to the expansion of solution (2.5.24) depends on the value of $k_{i}$. If $k_{i}=0$, the second approximation is $y^{*}=c_{1 i}^{*} x^{*}+c_{0 i}^{*}$. The coefficient is given by the formula

$$
c_{0 i}^{*}=\alpha_{0 i}^{*}+(-1)^{i} \sqrt{\frac{1-2 d}{2 a}} \frac{\left(\sqrt{2 a}+(-1)^{i} \sqrt{1-2 d}\right)^{2}+2 b+2 c^{*}-1}{4} \ln x^{*},
$$

where $\alpha_{0 i}^{*}$ is an arbitrary constant.

We also have a one-parameter family

$$
\mathcal{G}_{3}: \quad y^{*}=\varphi_{1}^{*} x^{*}+\sum_{\sigma^{*}=0}^{\infty} \varphi_{-\sigma^{*}}^{*} x^{*-\sigma^{*}}
$$

where

$$
\varphi_{1}^{*}=\frac{4}{1-2 a-2 d} \frac{1}{\ln ^{2} x^{*}}+\frac{c_{-3}^{*}}{\ln ^{3} x^{*}}+\sum_{s^{*}=4}^{\infty} \frac{c_{-s^{*}}^{*}}{\ln ^{s^{*}} x^{*}} .
$$

The complex coefficients are as follows: $c_{-3}^{*}$ is an arbitrary constant, and $c_{-s^{*}}^{*}$ are uniquely determined constants. $\varphi_{\sigma^{*}}^{*}$ are series in decreasing powers of logarithms.

Expansions of solutions for $a=-d+\frac{1}{2} \neq 0$. We have two one-parameter families of complicated expansions:

$$
\mathcal{G}_{3+j}: \quad y^{*}=\phi_{1 j}^{*} x^{*}+\sum_{\sigma^{*}=0}^{\infty} \phi_{-\sigma^{*} j}^{*} x^{*-\sigma^{*}}, \quad j=1,2,
$$

where

$$
\phi_{1 j}^{*}=(-1)^{j} \frac{1}{\sqrt{2 a}} \frac{1}{\ln x^{*}}+\frac{c_{-2 j}^{*}}{\ln ^{2} x^{*}}+\sum_{s^{*}=3}^{\infty} \frac{c_{-s^{*} j}^{*}}{\ln ^{s^{*}} x^{*}},
$$

and the complex coefficients are as follows: $c_{-2 j}^{*}$ is an arbitrary constant, and $c_{-s^{*} j}^{*}$ are uniquely determined constants. Also, $\phi_{\sigma^{*} j}^{*}$ are series in decreasing powers of logarithms.

The families of expansions $\mathcal{G}_{2}$ and $\mathcal{G}_{2}^{\tau}$ come from the case $a \neq-d+\frac{1}{2} \neq 0$. For $\theta_{2}^{*}$ we take $2 \sqrt{2 a}$ with $\operatorname{Re} \sqrt{2 a} \leq 0$. Depending on the value of $\theta_{2}^{*}$, three cases are possible: Case $1\left(\operatorname{Re} \theta_{2}^{*}=0\right.$, one-parameter families of exotic expansions $\mathcal{G}_{2}^{\tau}$ are given by formula $(2.5 .24)$ ), Case $2\left(\operatorname{Re} \theta_{2}^{*} \neq 0, \theta_{2}^{*} \notin \mathbb{Z}\right.$, the one-parameter family of power expansions $\mathcal{G}_{2}$ is given by formula (2.5.27) $)$, Case $3\left(\theta_{2}^{*} \notin \mathbb{Z}\right.$, the one-parameter family of power-logarithmic expansions $\mathcal{G}_{2}$ is given by formula (2.5.28) ). 
Expansions of solutions for $d=\frac{1}{2}, a \neq 0$. In the case $\operatorname{Re} \sqrt{2 a}<0$ we have the following one-parameter family of power expansions:

$$
\mathcal{G}_{6}: \quad y^{*}=x^{*}+c_{\rho^{*}}^{*} x^{* \rho^{*}}+\sum_{s^{*}} c_{s^{*}}^{*} x^{* s^{*}},
$$

where $\rho^{*}=1+\sqrt{2 a}, s^{*}$ runs over the set $\left\{\rho^{*}+l\left(\rho^{*}-1\right)-m ; l, m \geq 0 ; l+m>0\right.$; $l, m \in \mathbb{Z}\}$, and the complex coefficients are as follows: $c_{\rho^{*}}^{*}$ is an arbitrary nonzero constant, and the remaining $c_{s^{*}}^{*}$ are uniquely determined constants.

For $\operatorname{Re} \rho^{*}<0$ the third approximation to expansion (2.5.34) is $y=x^{*}+c_{\rho^{*}}^{*} x^{* \rho^{*}}+$ $c_{\rho^{*}-1}^{*} x^{* \rho^{*}-1}$. The coefficient is given by the formula

$$
c_{\rho^{*}-1}^{*}=c_{\rho^{*}}^{*} \frac{1-2(a+b+c)}{2\left(\rho^{*}-2\right)} .
$$

For $0<\operatorname{Re} \rho^{*}<1$ the third approximation to expansion (2.5.34) is $y^{*}=x^{*}+c_{\rho^{*}}^{*} x^{* \rho^{*}}+$ $c_{2 \rho^{*}-1}^{*} x^{* 2 \rho^{*}-1}$. The coefficient is given by the formula

$$
c_{2 \rho^{*}-1}^{*}=c_{\rho^{*}}^{* 2} .
$$

For $\operatorname{Re} \rho^{*}=0, \operatorname{Im} \rho^{*} \neq 0$ the third approximation to expansion (2.5.34) is $y^{*}=x^{*}+$ $c_{\rho^{*}}^{*} x^{* \rho^{*}}+c_{\rho^{*}-1}^{*} x^{* \rho^{*}-1}+c_{2 \rho^{*}-1}^{*} x^{* 2 \rho^{*}-1}$, where the coefficients $c_{\rho^{*}-1}^{*}$, and $c_{2 \rho^{*}-1}^{*}$ are given by formulas (2.5.35) and (2.5.36) respectively. For $\rho^{*}=0$ the third approximation to expansion (2.5.34) is $y^{*}=x^{*}+c_{0}^{*}+c_{-1}^{*} x^{*-1}$. The coefficients are as follows: $c_{0}^{*}$ is an arbitrary nonzero constant, and $c_{-1}^{*}=c_{0}^{*} \frac{\left(-1+2(a+b+c)+4 c_{0}^{*}\right)}{4}$ is the sum of expressions (2.5.35) and (2.5.36).

In the case $\operatorname{Re} \sqrt{2 a}=0$ there are two one-parameter families of exotic expansions:

$$
\mathcal{G}_{6}^{\tau}: \quad y^{*}=x^{*}+c_{\rho^{*}}^{*} x^{* \rho^{*}}+\sum_{s^{*}} c_{s^{*}}^{*} x^{* s^{*}}
$$

where $\rho^{*}=1+\sqrt{2 a}, s^{*}$ runs over the set $\left\{\rho^{*}+l\left(\rho^{*}-1\right)-m ; l, m \geq 0 ; l+m>0 ; l, m \in \mathbb{Z}\right\}$, $\tau= \pm 1$, and the complex coefficients are as follows: $c_{\rho^{*}}^{*}$ is an arbitrary nonzero constant, and all $c_{s^{*}}^{*}$ are uniquely determined constants.

Also, there exists a one-parameter family of complicated expansions $\mathcal{G}_{3}$. It comes from the case $a \neq \frac{1}{2}-d \neq 0$ and is given by formula (2.5.30).

\subsection{Summary of results.}

Theorem 2.5.1. As $x \rightarrow \infty$ and $a \cdot b \neq 0$, equation (2.1.1) has 13 families of expansions of solutions of Types $1-3$ :

$\mathcal{A}_{\infty}=\mathcal{A}_{0}^{*}$ is given by formula (2.5.5) and has 2 parameters;

$\mathcal{D}_{1}=\mathcal{B}_{1}^{*}$, which exists for $-b \neq c \neq 0$, is given by formulas (2.5.12), (2.5.13), and has 1 parameter;

$\mathcal{D}_{2}=\mathcal{B}_{2}^{*}$, which exists for $c \neq 0$, is given by formulas (2.5.12), (2.5.13), and has 1 parameter;

$\mathcal{D}_{3}=\mathcal{B}_{3}^{*}$, which exists for $-b \neq c$, is given by the formulas (2.5.15), (2.5.16) and has 1 parameter;

$\mathcal{D}_{4}=\mathcal{B}_{4}^{*}$ and $\mathcal{D}_{5}=\mathcal{B}_{5}^{*}$, which exist for $-b=c \neq 0$, are given by formulas (2.5.17), (2.5.18) and have 1 parameter;

$\mathcal{D}_{6}=\mathcal{B}_{6}^{*}$, which exists for $c=0$, is given by formula (2.5.19) and has 1 parameter; $\mathcal{G}_{1}=\mathcal{H}_{1}^{*}$, which exists for $a \neq-d+\frac{1}{2} \neq 0$, is given by formulas (2.5.27), (2.5.28), and has 1 parameter;

$\mathcal{G}_{2}=\mathcal{H}_{2}^{*}$, which exists for $d \neq \frac{1}{2}$, is given by formulas (2.5.27), (2.5.28), and has 1 parameter; 
$\mathcal{G}_{3}=\mathcal{H}_{3}^{*}$, which exists for $a \neq-d+\frac{1}{2} \neq 0$, is given by formulas (2.5.30), (2.5.31) and has 1 parameter;

$\mathcal{G}_{4}=\mathcal{H}_{4}^{*}$ and $\mathcal{G}_{5}=\mathcal{H}_{5}^{*}$, which exist for $a=-d+\frac{1}{2} \neq 0$, are given by formulas (2.5.32), 2.5.33) and have 1 parameter;

$\mathcal{G}_{6}=\mathcal{H}_{6}^{*}$, which exists for $d=\frac{1}{2}$, is given by formula (2.5.34) and has 1 parameter;

one family of semiexotic expansions:

$\mathcal{D}_{0}=\mathcal{B}_{0}^{\tau *}$, which exists for $b \neq 0$, is given by formula (2.5.8) and has 2 parameters;

three pairs of families of semiexotic expansions with $\tau= \pm 1$ :

$\mathcal{D}_{1}^{\tau}=\mathcal{B}_{1}^{\tau *}$, which exists for $-b \neq c \neq 0, \operatorname{Re}(\sqrt{2 c}-\sqrt{-2 b})=0$, is given by formula (2.5.9) and has 1 parameter;

$\mathcal{D}_{2}^{\tau}=\mathcal{B}_{2}^{\tau *}$, which exist for $b \neq 0, c \neq 0, \operatorname{Re}(\sqrt{2 c}+\sqrt{-2 b})=0$, are given by formula (2.5.9) and have 1 parameter;

$\mathcal{D}_{6}^{\tau}=\mathcal{B}_{6}^{\tau *}$, which exist for $b \neq 0, c=0, \operatorname{Re} \sqrt{-2 b}=0$, are given by formula (2.5.22) and have 1 parameter;

and four pairs of exotic expansions of solutions with $\tau= \pm 1$ :

$\mathcal{G}_{0}^{\tau}=\mathcal{H}_{0}^{\tau *}$, which exist for $a \neq 0$, are given by formula (2.5.23) and have 2 parameters;

$\mathcal{G}_{1}^{\tau}=\mathcal{H}_{1}^{\tau *}$, which exist for $a \neq-d+\frac{1}{2} \neq 0, \operatorname{Re}(\sqrt{1-2 d}-\sqrt{2 a})=0$, are given by formula (2.5.24) and have 1 parameter;

$\mathcal{G}_{2}^{\tau}=\mathcal{H}_{2}^{\tau *}$, which exist for $a \neq 0, d \neq \frac{1}{2}, \operatorname{Re}(\sqrt{1-2 d}+\sqrt{2 a})=0$, are given by formula (2.5.24) and have 1 parameter;

$\mathcal{G}_{6}^{\tau}=\mathcal{H}_{6}^{\tau *}$, which exist for $a \neq 0, d=\frac{1}{2}, \operatorname{Re} \sqrt{2 a}=0$, are given by formula (2.5.37) and have 1 parameter.

Here $\mathcal{A}_{0}^{*}, \mathcal{B}_{i}^{*}$ and $\mathcal{H}_{i}^{*}$ denote the families obtained from $\mathcal{A}_{0}, \mathcal{B}_{i}$ and $\mathcal{H}_{i}$ using the symmetry (2.1.6).

Families $\mathcal{D}_{1}, \mathcal{D}_{2}, \mathcal{D}_{6}, \mathcal{G}_{1}, \mathcal{G}_{2}, \mathcal{G}_{6}$ are power or power-logarithmic; families $\mathcal{D}_{3}, \mathcal{D}_{4}, \mathcal{D}_{5}$, $\mathcal{G}_{3}, \mathcal{G}_{4}, \mathcal{G}_{5}$ are complicated.

Chapter 3. Expansions of SOlutions of P6 NEAR ZERO AND INFINITY IN THE CASES $a=0, b \neq 0$ AND $a \neq 0, b=0$

\section{General properties of the equation}

1.1. Formulation of the problem. In this chapter we are looking for asymptotic expansions of the form (2.1.2) for solutions of the sixth Painlevé equation (2.1.1). We are looking for expansions of all five types: power, power-logarithmic, complicated, semiexotic, and exotic, as $x \rightarrow 0$ and $x \rightarrow \infty$ in the following two cases:

1) $a=0, b \neq 0, c$ and $d$ are arbitrary;

2) $a \neq 0, b=0, c$ and $d$ are arbitrary.

1.2. Supports and normal cones. In the case $a=0$ equation (2.1.4) takes the form:

$$
\begin{aligned}
f(x, y) \stackrel{\text { def }}{=} & 2 y^{\prime \prime} x^{2}(x-1)^{2} y(y-1)(y-x) \\
& -\left(y^{\prime}\right)^{2}\left[x^{2}(x-1)^{2}(y-1)(y-x)+x^{2}(x-1)^{2} y(y-x)+x^{2}(x-1)^{2} y(y-1)\right] \\
& +2 y^{\prime}\left[x(x-1)^{2} y(y-1)(y-x)+x^{2}(x-1) y(y-1)(y-x)+x^{2}(x-1)^{2} y(y-1)\right] \\
& -\left[2 b x(y-1)^{2}(y-x)^{2}+2 c(x-1) y^{2}(y-x)^{2}+2 d x(x-1) y^{2}(y-1)^{2}\right]=0 .
\end{aligned}
$$



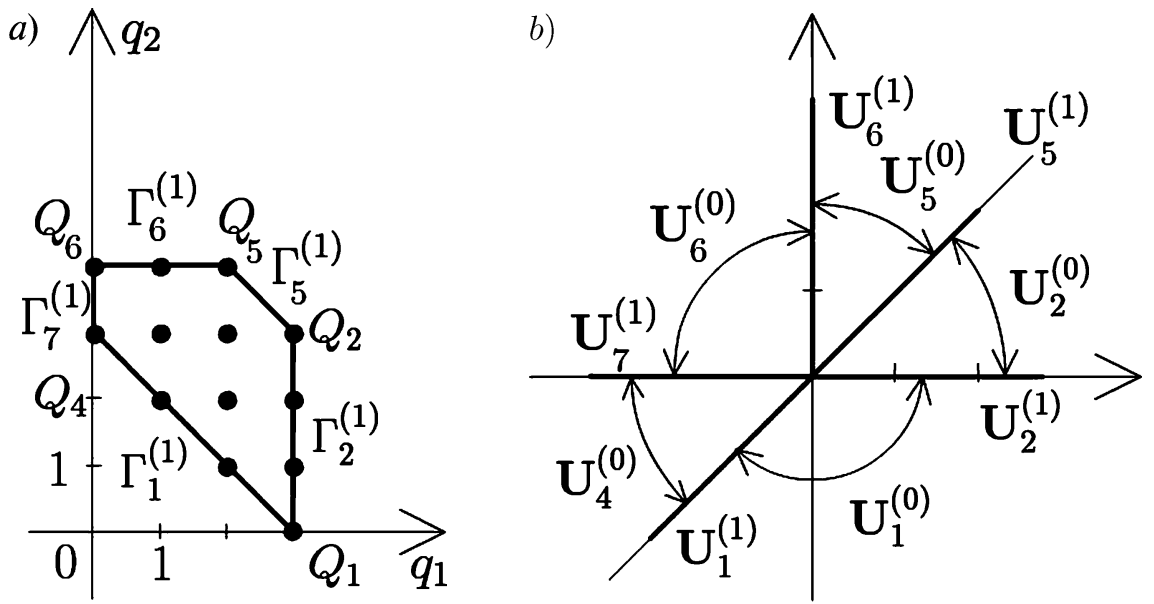

Figure 14. For equation (3.1.1): (a) the support, the polygon, and its faces; (b) normal cones of faces.

In this case the support is obtained from the one shown in Figure $8(a)$ by removing the points $(0,6),(0,5),(1,5)$. The support $\mathbf{S}\left(f_{a=0}\right)$ of the left-hand side of equation (3.1.1), its convex hull $\Gamma\left(f_{a=0}\right)$, faces $\Gamma_{i}^{(0)} \stackrel{\text { def }}{=} Q_{i}, i=1,2,4,5,6, \Gamma_{j}^{(1)}, j=1,2,5,6,7$, are shown in Figure 14 $(a)$ (faces of the polygon $\Gamma(f)$, faces coming from the case $a \cdot b \neq 0$ preserve their numbers). Real normal cones $\mathbf{U}_{i}^{(d)}, d=0,1$ corresponding to these are shown in Figure $14(b)$.

For $\omega=-1$ the decomposition of the complex plane $L_{-}: r \in \mathbb{C}$ into complex normal cones $\breve{\mathbf{U}}_{j}^{(d)}$ is shown in Figure 5 with the following change on notation: $\breve{\mathbf{U}}_{6}^{(0)}$ and $\breve{\mathbf{U}}_{7}^{(1)}$ should be used instead of $\breve{\mathbf{U}}_{3}^{(0)}$ and $\breve{\mathbf{U}}_{4}^{(1)}$ respectively. This decomposition is similar to the case $a \neq 0$.

For $b=0$, equation (2.1.4 takes the form

$$
\begin{aligned}
f(x, y) \stackrel{\text { def }}{=} & 2 y^{\prime \prime} x^{2}(x-1)^{2} y(y-1)(y-x) \\
& -\left(y^{\prime}\right)^{2}\left[x^{2}(x-1)^{2}(y-1)(y-x)+x^{2}(x-1)^{2} y(y-x)+x^{2}(x-1)^{2} y(y-1)\right] \\
& +2 y^{\prime}\left[x(x-1)^{2} y(y-1)(y-x)+x^{2}(x-1) y(y-1)(y-x)+x^{2}(x-1)^{2} y(y-1)\right] \\
& -\left[2 a y^{2}(y-1)^{2}(y-x)^{2}+2 c(x-1) y^{2}(y-x)^{2}+2 d x(x-1) y^{2}(y-1)^{2}\right]=0 .
\end{aligned}
$$

In this case the support is obtained from the one shown in Figure 8 $(a)$ by removing the points $(3,0),(2,1),(3,1)$. The support $\mathbf{S}\left(f_{b=0}\right)$ of the left-hand side of equation (3.1.2), its convex hall $\Gamma\left(f_{b=0}\right)$, faces $\Gamma_{i}^{(0)} \stackrel{\text { def }}{=} Q_{i}, i=2,3,4,7,8, \Gamma_{j}^{(1)}, j=3,4,8,9,10$, are shown in Figure 15] (a) (the faces of the polygon $\Gamma(f)$ coming from the case $a \cdot b \neq 0$ preserve their numbers), real normal cones $\mathbf{U}_{i}^{(d)}, d=0,1$, corresponding to these faces are shown in Figure 15) (b).

Using the symmetry (2.1.6) one can reduce computations. Namely, this symmetry sends the case $a=0$ to the case $b=0$. Under this symmetry, vertices $\Gamma_{i}^{(0)}, i=1,2,4,5,6$, and edges $\Gamma_{j}^{(1)}, j=1,2,5,6,7$, in the case $a=0$ correspond to the vertices $\Gamma_{i}^{(0)}, i=$ $3,4,2,7,8$, and edges $\Gamma_{j}^{(1)}, j=3,4,8,9,10$, in the case $b=0$.

Therefore, we will consider in detail only the case $a=0$, and then extend the obtained results to the case $b=0$ using symmetry (2.1.6). 
a)

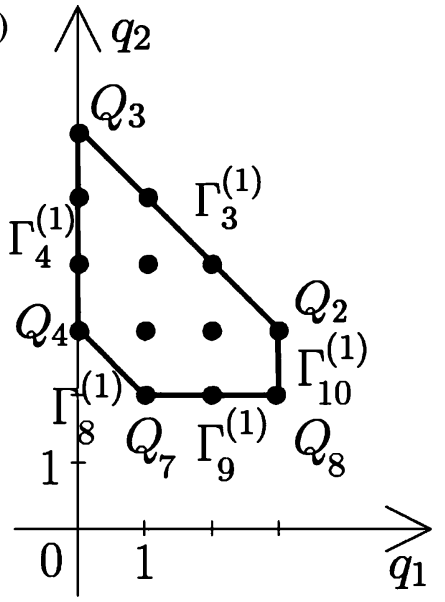

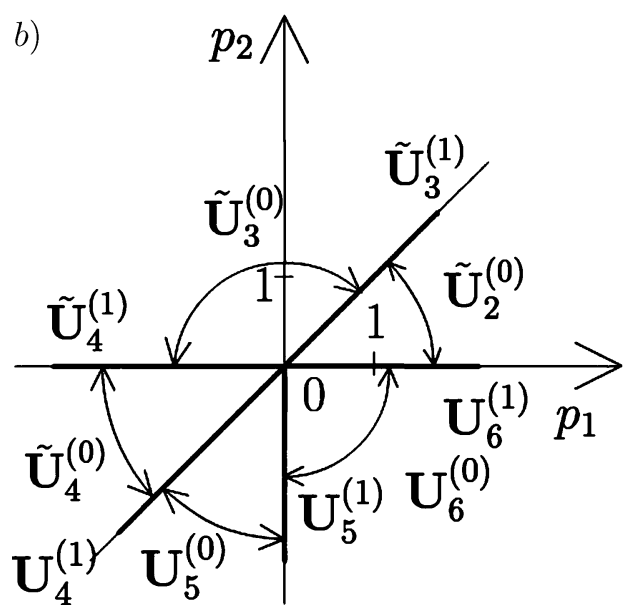

Figure 15. For equation (3.1.2): (a) the support, the polygon, and its faces; (b) normal cones of faces.

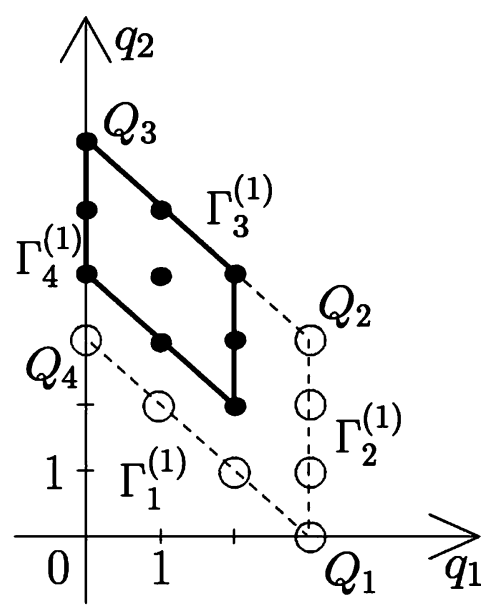

Figure 16. The support of terms of equation (2.1.4) with coefficient $a$.

Furthermore, the vertices $\Gamma_{1}^{(0)}, \Gamma_{2}^{(0)}, \Gamma_{3}^{(0)}, \Gamma_{4}^{(0)}$ and the edges $\Gamma_{1}^{(1)}, \Gamma_{2}^{(1)}, \Gamma_{3}^{(1)}$, and $\Gamma_{4}^{(1)}$ shown in Figures $8(a)$, 14 $(a)$, and 15) ( $a$ ) were considered in the case $a \cdot b \neq 0$ in Chapter 2. The terms of equation (2.1.4) with the parameter $a$ as a factor have the support shown by solid dots in Figure 16 .

Therefore, for $a=0$ the terms of equation (2.1.4) corresponding to the vertices $\Gamma_{1}^{(0)}$, $\Gamma_{2}^{(0)}, \Gamma_{4}^{(0)}$ and the edges $\Gamma_{1}^{(1)}, \Gamma_{2}^{(1)}$ do not change, and neither do the corresponding reduced equations. Hence the corresponding expansions retain their general structure and may only change starting with the second term.

For $b=0$, similarly to the case $a=0$ the terms of equation (2.1.4) corresponding to the vertices $\Gamma_{2}^{(0)}, \Gamma_{3}^{(0)}, \Gamma_{4}^{(0)}$ and the edges $\Gamma_{3}^{(1)}, \Gamma_{4}^{(1)}$ do not change. Therefore the corresponding expansions retain their general structure and may only change starting with the second term.

Therefore, in the case $a=0$ we consider the vertices $\Gamma_{5}^{(0)}, \Gamma_{6}^{(0)}$ and edges $\Gamma_{5}^{(1)}, \Gamma_{6}^{(1)}$, $\Gamma_{7}^{(1)}$ for which the types of expansions of solutions of (2.1.1) may be new. 
Similarly, in the case $b=0$ new types of expansions may only correspond to vertices $\Gamma_{7}^{(0)}, \Gamma_{8}^{(0)}$ and edges $\Gamma_{8}^{(1)}, \Gamma_{9}^{(1)}, \Gamma_{10}^{(1)}$; we obtain these expansions using the symmetry (2.1.6) and results for the case $a=0$.

Furthermore, using the symmetry (2.1.8) one can reduce computations even in the case $a=0$ since this symmetry sends expansions corresponding to the vertex $\Gamma_{5}^{(0)}$ and the edges $\Gamma_{5}^{(1)}, \Gamma_{6}^{(1)}$ to expansions corresponding to the vertex $\Gamma_{6}^{(0)}$ and the edges $\Gamma_{7}^{(1)}$, $\Gamma_{6}^{(1)}$ (and vice versa).

Therefore, we need to study in detail one vertex $\Gamma_{6}^{(0)}$ and two edges $\Gamma_{6}^{(1)}, \Gamma_{7}^{(1)}$ in the case $a=0$, and then using symmetries (2.1.6), (2.1.8) to pass obtained results to all remaining cases. Namely, in the case $a=0$ we use symmetry (2.1.8) to obtain expansions of solutions corresponding to the vertex $\Gamma_{5}^{(0)}$ and the edge $\Gamma_{5}^{(1)}$, and then use symmetry (2.1.6) we obtain expansions for $b=0$ from expansions for $a=0$.

Let us recall that according to Theorem 2.1.4 in the case $a=0$ there is an exceptional solution $\mathcal{I}_{4}: y=\infty$, and in the case $b=0$, solution $\mathcal{I}_{1}: y=0$.

\section{EXPANSIONS NEAR ZERO CORRESPONDING TO THE VERTEX $\Gamma_{6}^{(0)}$}

The vertex $\Gamma_{6}^{(0)} \stackrel{\text { def }}{=} Q_{6}=(0,4)$ is the upper left vertex in Figure 14 $(a)$, the corresponding value of $\omega$ is $\omega=-1$ and the reduced equation is

$$
\hat{f}_{6}^{(0)}(x, y) \stackrel{\text { def }}{=} 2 y^{\prime \prime} x^{2} y^{3}-3 y^{\prime 2} x^{2} y^{2}+2 y^{\prime} x y^{3}+2 c y^{4}=0 .
$$

The real normal cone is $\mathbf{U}_{6}^{(0)}=\left\{p_{1}<0, p_{2}>0\right\}$, i.e., $x \rightarrow 0, y \rightarrow \infty$. The complex normal cone is $\breve{\mathbf{U}}_{6}^{(0)}=-(1, r)$, where

$$
r: \quad r \in \mathbb{C}, \operatorname{Re} r<0 .
$$

2.1. Expansions of solutions with power asymptotics. A solution of equation (3.2.1) is the expression $y=c_{r} x^{r}$, where $c_{r}$ is an arbitrary nonzero constant. The exponent $r$ is determined from the characteristic equation $\chi(r)=x^{-4 r} \hat{f}_{6}^{(0)}\left(x, x^{r}\right)=$ $-r^{2}+2 c=0$, which has two roots

$$
r_{1,2}= \pm \sqrt{2 c}
$$

and from the consideration of the complex normal cone (3.2.2).

Let us compute the critical numbers. The first variation is

$$
\begin{aligned}
\frac{\partial \hat{f}_{6}^{(0)}(x, y)}{\partial y}= & 2 \frac{d^{2}}{d x^{2}} x^{2} y^{3}+6 y^{\prime \prime} x^{2} y^{2}-6 y^{\prime} \frac{d}{d x} x^{2} y^{2} \\
& -6 y^{\prime 2} x^{2} y+2 \frac{d}{d x} x y^{3}+6 y^{\prime} x y^{2}+8 c y^{3} .
\end{aligned}
$$

The linear differential operator is

$$
\mathcal{L}(x) \stackrel{\text { def }}{=} 2 c_{r}^{3} x^{3 r}\left(\frac{d^{2}}{d x^{2}} x^{2}+3 r(r-1)-3 r \frac{d}{d x} x-3 r^{2}+\frac{d}{d x} x+3 r+4 c\right) .
$$

The characteristic polynomial

$$
\nu(k)=2 c_{r}^{3}\left(k^{2}-3 r k+2 r^{2}\right)
$$

has two roots $k_{1}=2 r$ and $k_{2}=r$. The cone of the problem is $\mathcal{K}=\{\operatorname{Re} k>\operatorname{Re} r\}$. The numbers $k_{1,2}$ do not belong to $\mathcal{K}$; therefore, there are no critical numbers.

The support of the expansions of the solution is

$$
\mathbf{K}=\{s=r-l r+m ; l \geq 0, m \geq 1 ; l, m \in \mathbb{Z}\} .
$$


Therefore, we obtain a one-parameter family of expansions

$$
\mathcal{C}_{0}^{\infty}: \quad y=c_{r} x^{r}+\sum_{s} c_{s} x^{s}
$$

where the exponents are as follows: $r= \pm \sqrt{2 c}$ and satisfies inequality (3.2.2), $s$ belongs to the set (3.2.7), $c_{r}$ is an arbitrary nonzero constant, and all $c_{s}$ are uniquely determined constants.

The support (3.2.7) has two generators $-r$ and 1 and on the complex plane it is located in the sector with vertex at $r$ and sides that are parallel to vectors $-(\operatorname{Re} r, \operatorname{Im} r)$ and $(1,0)$.

Therefore, we have proved the following result.

Theorem 3.2.1. For $x \rightarrow 0$ and $a=0, c \neq 0$, there exists a one-parameter family of power expansions $\mathcal{C}_{0}^{\infty}$ of solutions; this family is given by formula (3.2.8).

By Theorem 1.7.2 the series (3.2.8) converges for sufficiently small $|x|$.

If $2 c=k^{2}$, where $k$ is an integer, then, according to (3.2.7), expansion (3.2.8) contains only integral powers of $x$, i.e., is a Laurent series. This is the only case where this family was previously known (see [54, §46, Theorem 46.3]).

Let us compute the second approximation to solutions.

If $\operatorname{Re} r \leq-1$, the second approximation is $y=c_{r} x^{r}+c_{r+1} x^{r+1}$. Let us compute the coefficient $c_{r+1}$. The second approximation to equation (3.1.1) is

$$
\hat{\hat{f}}_{6}^{(0)}(x, y)=-4 y^{\prime \prime} x^{3} y^{3}+6{y^{\prime}}^{2} x^{3} y^{2}-6 y^{\prime} x^{2} y^{3}+2 x y^{4}(-c+d-b) .
$$

Since $b_{r+1}=x^{-4 r-1} \hat{\hat{f}}_{6}^{(0)}\left(x, c_{r} x^{r}\right)=2 c_{r}^{4}\left(r^{2}-r-c+d-b\right), \nu(1+r)=2 c_{r}^{3}(1-r)$, we have $c_{r+1}=\frac{c_{r}(c+d-b-r)}{(r-1)}$.

If $-1<\operatorname{Re} r<0$, then the second approximation to the solution is $y=c_{r} x^{r}+c_{0}$ and the second approximation to equation (3.1.1) is

$$
\hat{\hat{f}}_{6}^{(0)}(x, y)=-2 y^{\prime \prime} x^{2} y^{2}+2 y^{\prime 2} x^{2} y-2 y^{\prime} x y^{2} .
$$

Since $b_{0}=\hat{\hat{f}}_{6}^{(0)}\left(x, c_{r} x^{r}\right)=0, \nu(0)=4 c_{r}^{3} r^{2} \neq 0$, we have $c_{0}=0$.

The case $\operatorname{Re} r=-1$ will be considered together with the case $\operatorname{Re} \sqrt{2 c} \leq-1$ because the contribution of the second approximation to (3.2.10) vanishes.

2.2. Nonpower asymptotics. Let us compute nonpower solutions of equation (3.2.1) if they exist. Let us transform this equation as follows:

$$
g(x, y)=y^{-4} \hat{f}_{6}^{(0)}(x, y)=\frac{2 y^{\prime \prime}}{y} x^{2}-\frac{3 y^{\prime 2}}{y^{2}} x^{2}+\frac{2 y^{\prime}}{y} x+2 c=0,
$$

$\mathbf{S}(g)=\{0\}$. Equation $g(x, y)=0$ contains a nonzero constant $2 c$. In the case $c=0$ equation $\chi(r)=0$ has a double root $r=0$. But $P=\omega(1,0)$ does not belong to the normal cone $\mathbf{U}_{6}^{(0)}$. Since $\Delta(g)=2$ and

$$
g^{*}=\frac{2 y^{\prime \prime}}{y} x^{2}-\frac{3 y^{\prime 2}}{y^{2}} x^{2}, \quad \operatorname{coef}\left(g^{*}\right)=2-3=-1 \neq 0,
$$

by Theorems 1.3.5 and 1.3.6 there are no appropriate nonpower asymptotics. 


\section{EXPANSIONS CORRESPONDING TO THE VERTEX $\Gamma_{6}^{(1)}$}

The reduced equation corresponding to the edge $\Gamma_{6}^{(1)}$ is

$$
\begin{aligned}
\hat{f}_{6}^{(1)}(x, y) \stackrel{\text { def }}{=} & 2 y^{\prime \prime} x^{4} y^{3}-3 y^{\prime 2} x^{4} y^{2}+4 y^{\prime} x^{3} y^{3}-2 d x^{2} y^{4} \\
& +2 y^{\prime \prime} x^{2} y^{3}-3 y^{\prime 2} x^{2} y^{2}+2 y^{\prime} x y^{3}+2 c y^{4}-4 y^{\prime \prime} x^{3} y^{3} \\
& +6 y^{\prime 2} x^{3} y^{2}-2 x y^{4}(c+b-d)-6 y^{\prime} x^{2} y^{3}=0 .
\end{aligned}
$$

It does produce power expansions since the edge $\Gamma_{6}^{(1)}$ is horizontal. Let us check if there are nonpower asymptotics. The edge $\Gamma_{6}^{(1)}$ joins the vertices $Q_{6}=(0,4)$ and $Q_{5}=(2,4)$, and the corresponding reduced equations $\hat{f}_{6}^{(0)}(x, y) \stackrel{\text { def }}{=} 2 y^{\prime \prime} x^{2} y^{3}-3 y^{\prime 2} x^{2} y^{2}+2 y^{\prime} x y^{3}+$ $2 c y^{4}=0$ and $\hat{f}_{5}^{(0)}(x, y) \stackrel{\text { def }}{=} 2 y^{\prime \prime} x^{4} y^{3}-3 y^{\prime 2} x^{4} y^{2}+4 y^{\prime} x^{3} y^{3}-2 d x^{2} y^{2}=0$ have the order of differentiation equal to 2 . On the other hand, the order of differentiation of equation $\hat{f}_{6}^{(1)}(x, y)=0$ also equals 2 . Therefore, by Theorem 1.3.4 required solutions do not exist in either case $x \rightarrow 0$ or $x \rightarrow \infty$.

\section{Expansions CORRESPonding to the EdGe $\Gamma_{7}^{(1)}$}

4.1. Preliminary analysis. The vertex $\Gamma_{7}^{(1)}$ is vertical. The corresponding reduced equation is

$$
\hat{f}_{7}^{(1)}(x, y) \stackrel{\text { def }}{=} 2 y^{\prime \prime} x^{2} y^{3}-3 y^{\prime 2} x^{2} y^{2}+2 y^{\prime} x y^{3}+2 c y^{4}+2 y^{\prime 2} x^{2} y-2 y^{\prime \prime} x^{2} y^{2}-2 y^{\prime} x y^{2}=0
$$

and $r=0$. The real normal cone is $\mathbf{U}_{7}^{(1)}=\{\lambda(-1,0), \lambda>0\}$, i.e. $x \rightarrow 0$ and $\omega=-1$.

The reduced equation (3.4.1) is the sum of two reduced equations (2.2.1) and (3.2.1). For $\rho^{2} \neq 2 c$ the number $\rho=r+i \gamma$ with $r=0, \gamma \neq 0, \gamma \in \mathbb{R}$ is a root of the characteristic equation $\chi(\rho) \equiv 0$ corresponding to the bottom vertex $\Gamma_{4}^{(0)}=(0,3)$. By Theorem 1.5.1 we have two families of exotic expansions $\mathcal{B}_{7}^{\tau}$ with $\tau=\operatorname{sgn} \operatorname{Im} \rho$. The cones of the problem are $\mathcal{K}^{\tau}=\{s: \operatorname{Re} s \geq 0$, and $\operatorname{Re} s=0, \operatorname{sgn} \operatorname{Im} s=\tau\}$. Since the characteristic equation (2.2.4) has the root $k_{1,2}=\rho$, which does not belong to the cone of the problem, there are no critical numbers. The support of expansions of solutions is

$$
\mathbf{K}=\{\rho+l \rho+m(1-\rho) ; l, m \geq 0 ; l+m>0 ; l, m \in \mathbb{Z}\} .
$$

Therefore, we have two families of exotic expansions

$$
\mathcal{B}_{7}^{\tau}: \quad y=c_{\rho} x^{\rho}+\sum c_{s} x^{s} \quad(\text { sum over } s \in \mathbf{K}),
$$

where $\tau=\operatorname{sgn} \operatorname{Im} \rho, \rho^{2} \neq 2 c, \operatorname{Re} \rho=0, \rho \neq 0, s$ runs over the set (3.4.2), $c_{r}$ is an arbitrary nonzero complex constant, and all complex coefficients $c_{s}$ are uniquely determined constants.

A more precise form of these families is given in (3.4.39).

If $\rho^{2}=2 c, \operatorname{Re} \rho=0, \rho \neq 0$, we have the case described in Remark 1.5.1. The number $\rho=r+i \gamma$ with $r=0, \gamma \neq 0, \gamma \in \mathbb{R}$ is a root of both the characteristic equation $\chi(\rho) \equiv 0$ corresponding to the bottom vertex $\Gamma_{4}^{(0)}=(0,3)$, and the characteristic equation $\chi(\rho)=\rho^{2}-2 c=0$ corresponding to the top vertex $\Gamma_{6}^{(0)}=(0,4)$.

According to Remark 1.5.1 here we have two families of expansions with the initial term $y=c_{\rho} x^{\sqrt{2 c}}$. Namely, we have the family $\mathcal{B}_{8}$ with $\operatorname{Im} \sqrt{2 c}>0$ and the family $\mathcal{B}_{9}$ with $\operatorname{Im} \sqrt{2 c}<0$. In both cases the cone of the problem is $\mathcal{K}=\{s: \operatorname{Re} s>0\}$.

The characteristic equation (2.2.4) corresponding to the reduced solution $y=c_{\rho} x^{\rho}$ and the vertex $\Gamma_{4}^{(0)}=(0,3)$ has a double root $k_{1,2}=\rho$. Since it is not in the cone of the 
problem, there are no critical numbers. The support of expansions of solutions (2.2.6) corresponding to the vertex $\Gamma_{4}^{(0)}$ is

$$
\mathbf{K}_{1}=\{\rho+l \rho+m(1-\rho) ; l, m \geq 0 ; l+m>0 ; l, m \in \mathbb{Z}\} .
$$

The characteristic equation (3.2.6) corresponding to the reduced solution $y=c_{\rho} x^{\rho}$ and the vertex $\Gamma_{6}^{(0)}=(0,4)$ has two roots $k_{1}=\rho$ and $k_{2}=2 \rho$. Since neither of them is in the cone of the problem, there are no critical numbers. The support of expansions of solutions (3.2.8) corresponding to the vertex $\Gamma_{6}^{(0)}$ is

$$
\mathbf{K}_{2}=\{\rho-l \rho+m ; l \geq 0 ; m \geq 1 ; l, m \in \mathbb{Z}\} .
$$

According to Remark 1.5.1 we have two families of expansions

$$
\mathcal{B}_{8,9}: \quad y=c_{\rho} x^{\rho}+\sum_{\operatorname{Re} s \geq 1} c_{s} x^{s} \quad(\text { sum over } s \in \mathbf{K}),
$$

where $\rho$ is a purely imaginary constant, $\rho^{2}=2 c, \operatorname{Im} \rho>0$ for the family $\mathcal{B}_{8}$ and $\operatorname{Im} \rho<0$ for the family $\mathcal{B}_{9}$, and $s \in \mathbf{K}=\mathbf{K}_{1} \cap \mathbf{K}_{2}$, i.e.,

$$
s \in\{\rho+l(1-\rho)+m ; l, m \geq 0 ; l+m>0 ; l, m \in \mathbb{Z}\} .
$$

The complex coefficients are as follows: $c_{\rho}$ is an arbitrary nonzero constant, and the $c_{s}$ are uniquely determined constants.

Since $r=0$, we are looking for power solutions of equation (3.4.1) in the form $y=c_{0}$, $c_{0} \neq 0$. Let us compute $c_{0}$. The defining equation is

$$
\tilde{f}_{7}^{(1)}\left(c_{0}\right) \stackrel{\text { def }}{=} c_{0}^{-3} \hat{f}_{7}^{(1)}\left(x, c_{0}\right) \stackrel{\text { def }}{=} 2 c c_{0}=0 .
$$

The height of the edge $\Gamma_{7}^{(1)}$ equals 1 , and the defining equation is linear. This defining equation shows that for $c=0$ any constant $c_{0}$ is a solution, whereas for $c \neq 0$ the equation has only the zero solution $c_{0}=0$. Therefore, we consider two cases: $c \neq 0$ and $c=0$.

4.2. Expansion of solutions in the case $a=0, b \cdot c \neq 0$. In this case there are no power solutions of equation (3.4.1). Let us find nonpower solutions of this equation.

Since the defining equation does not have multiple nonzero solutions, by Theorem 1.3 .2 there are no solutions that tend to a nonzero constant.

In equation (3.4.1), let us make the logarithmic transform $\xi=\ln x$. Then, according to (2.2.13) and (3.4.1), we obtain the equation

$$
\varphi(\xi, y) \stackrel{\text { def }}{=} \hat{f}_{7}^{(1)}(\xi, y)=2 \ddot{y} y^{2}(y-1)+\dot{y}^{2} y(2-3 y)+2 c y^{4}=0 .
$$

By Theorem 1.3 .3 we are interested in only those solutions of this equation that tend to zero as $\xi \rightarrow \infty$. The support of the left-hand side of the equation, its convex hull, and normal cones of all faces are shown in Figure 17. The cone of the problem is $\mathcal{K}=\left\{p_{1} \geq 0, p_{2} \leq 0\right\}$, which corresponds to the limit $\xi \rightarrow \infty$. The real normal cones that have nonempty intersection with the cone of the problem are $\mathbf{U}_{1}^{(0)}, \mathbf{U}_{2}^{(0)}$, and $\mathbf{U}_{1}^{(1)}$. The only solution of the reduced equation $\hat{\varphi}_{2}^{(0)}(\xi, y) \stackrel{\text { def }}{=} 2 c y^{4}=0$ is a trivial solution.

The reduced equation corresponding to the vertex $\Phi_{1}^{(0)}$ is

$$
\hat{\varphi}_{1}^{(0)}(\xi, y) \stackrel{\text { def }}{=}-2 \ddot{y} y^{2}+2 \dot{y}^{2} y=0 .
$$

It has a solution $y=c_{0}$ because the vector $P=\omega(1, r)=(1,0)$ is in $\mathbf{U}_{1}^{(0)} \cap \mathcal{K}$. However, the constant solution does not work in our case.

The reduced equation corresponding to the edge $\Phi_{1}^{(1)}$ is

$$
\hat{\varphi}_{1}^{(1)}(\xi, y)=-2 \ddot{y} y^{2}+2 \dot{y}^{2} y+2 c y^{4}=0 .
$$



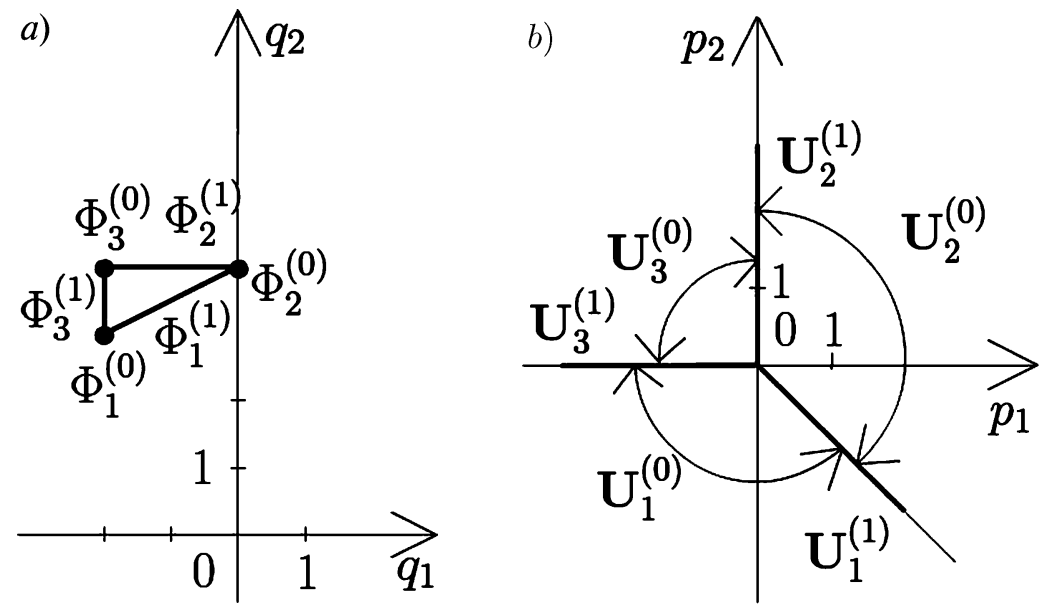

Figure 17. For equation (3.4.8): (a) the support, the polygon, and its faces; (b) normal cones of faces.

The real normal cone is $\mathbf{U}_{1}^{(1)}=\{\lambda(1,-2), \lambda>0\}$. The first approximation to a solution of the equation $\varphi(\xi, y)=0$ is $y=c_{-2} \xi^{-2}, c_{-2} \neq 0$. Let us compute the coefficient $c_{-2}$. The defining equation is

$$
\tilde{\varphi}_{1}^{(1)}\left(c_{-2}\right)=c_{-2}^{3}\left(-4+2 c c_{-2}\right)=0,
$$

and since $c_{-2} \neq 0$, it has a solution $c_{-2}=\frac{2}{c}$. Let us find critical numbers of this solution. The first variation is

$$
\frac{\partial \hat{\varphi}_{1}^{(1)}(\xi, y)}{\partial y}=-2 \frac{d^{2}}{d \xi^{2}} y^{2}-4 \ddot{y} y+4 \dot{y} \frac{d}{d \xi} y+2 \dot{y}^{2}+8 c y^{3} .
$$

The linear differential operator is

$$
\mathcal{L}(\xi)=-2 \frac{d^{2}}{d \xi^{2}} c_{-2}^{2} \frac{1}{\xi^{4}}-24 c_{-2}^{2} \frac{1}{\xi^{6}}-8 c_{-2}^{2} \frac{d}{d \xi} \frac{1}{\xi^{5}}+8 c_{-2}^{2} \frac{1}{\xi^{6}}+8 c c_{-2}^{3} \frac{1}{\xi^{6}} .
$$

The characteristic polynomial $\nu(k)=c_{-2}^{2}\left(-2 k^{2}-6 k\right), c_{-2} \neq 0$, has two roots, $k_{1}=0$ and $k_{2}=-3$. The cone of the problem is $\mathcal{K}=\{\operatorname{Re} k<-2$ or $\operatorname{Re} k=0, \operatorname{Im} k \neq 0\}$. Since $k_{2} \in \mathcal{K}$, we can see that $k_{2}$ is the only critical number. The support of the expansion of the solution is $\mathbf{K}=\{s=-2-2 l, l>0\}$. The set $\mathbf{K}\left(k_{2}\right)$ is $\mathbf{K}\left(k_{2}\right)=\{s=-2-l, l>0\}$. The second approximation to the solution of equation $\varphi(\xi, y)=0$ is

$$
y=c_{-2} \frac{1}{\xi^{2}}+c_{-3} \frac{1}{\xi^{3}} .
$$

Since $k_{2} \notin \mathbf{K}$, the compatibility condition is automatically satisfied and the coefficient $c_{-3}$ is an arbitrary complex constant.

The expansion of the solution is given by the formula

$$
y=\frac{2}{c} \frac{1}{\xi^{2}}+c_{-3} \frac{1}{\xi^{3}}+\sum_{s=4}^{+\infty} c_{-s} \frac{1}{\xi^{s}},
$$

where $c_{-3}$ is an arbitrary complex constant and $c_{-s}$ are uniquely determined constant complex coefficients. By Theorem 1.7.2, the series (3.4.15) converges for sufficiently large $|\xi|$. Making the inverse transformation, we obtain the asymptotics for the solution 
of equation (3.1.1),

$$
y=\frac{2}{c} \frac{1}{\ln ^{2} x}+c_{-3} \frac{1}{\ln ^{3} x}+\sum_{s=4}^{+\infty} c_{-s} \frac{1}{\ln ^{s} x}
$$

where $c_{-3}$ is an arbitrary complex constant and the complex coefficients $c_{-s}$ are uniquely determined constants.

Let us compute the critical numbers of the reduced solutions (3.4.16). The first variation is given by formula (3.4.19). Denote it by $\mathcal{M}(x, y)$. Let us make in $\mathcal{M}(x, y)$ the logarithmic transformation $\xi=\ln x$ and denote the differentiation with respect to $\xi$ by a dot. Then $y^{\prime}=\frac{\dot{y}}{x}, y^{\prime \prime}=\frac{(\dot{y}-\dot{y})}{x^{2}}$. After the transformation, we obtain the operator

$$
\begin{aligned}
\mathcal{M}(x, y)= & 2\left(\frac{d^{2}}{d \xi^{2}}-\frac{d}{d \xi}\right) y^{3}+6(\ddot{y}-\dot{y}) y^{2}-6 \frac{d}{d \xi} \dot{y} y^{2}-6 \dot{y}^{2} y+2 \frac{d}{d \xi} y^{3} \\
& +6 \dot{y} y^{2}+4 \frac{d}{d \xi} \dot{y} y+2 \dot{y}^{2}-2\left(\frac{d^{2}}{d \xi^{2}}-\frac{d}{d \xi}\right) y^{2} \\
& -4(\ddot{y}-\dot{y}) y-2 \frac{d}{d \xi} y^{2}-4 \dot{y} y+8 c y^{3} \stackrel{\text { def }}{=} \mathcal{N}(\xi, y) .
\end{aligned}
$$

For solutions of equation (3.4.16) we have $y=\frac{2}{c} \frac{1}{\xi^{2}}+\cdots$. Therefore, in the operator $\mathcal{N}$, the largest exponent $n$ in terms with $\xi^{n}$ is $n=-4$, and the sum of terms with $\xi^{-4}$ form the operator

$$
\mathcal{N}_{-4}=-2 y^{2} \frac{d^{2}}{d \xi^{2}}
$$

where $y=\frac{2}{c} \frac{1}{\xi^{2}}$. The characteristic polynomial corresponding to this operator is $\nu(k)=$ $-2\left(\frac{2}{c}\right)^{2} k^{2}$. This polynomial has a double root $k=0=r$; i.e., it does not produce critical values.

By Theorem 1.4.1 for solutions of the original equation (3.1.1) there exists a unique expansion

$$
\mathcal{B}_{3}: \quad y=\varphi_{0}+\sum_{\sigma=1}^{\infty} \varphi_{\sigma} x^{\sigma},
$$

where $\varphi_{0}$ is given by (3.4.15) and $\varphi_{\sigma}$ are series in decreasing powers of simple (noniterated) logarithms. This expansion is obtained from (2.3.30) and (2.3.28) for $a=0$.

4.3. Expansion of solutions for $a=c=0, b \neq 0$. First, we find power and powerlogarithmic expansion.

According to (3.4.7) the coefficient $c_{0}$ is an arbitrary constant. Let us compute critical numbers. The first variation is

$$
\begin{aligned}
\frac{\partial \hat{f}_{7}^{(1)}(x, y)}{\partial y}= & 2 \frac{d^{2}}{d x^{2}} x^{2} y^{3}+6 y^{\prime \prime} x^{2} y^{2}-6 \frac{d}{d x} y^{\prime} x^{2} y^{2}-6 y^{\prime 2} x^{2} y \\
& +2 \frac{d}{d x} x y^{3}+6 y^{\prime} x y^{2}+4 \frac{d}{d x} y^{\prime} x^{2} y+2 y^{\prime 2} x^{2}-2 \frac{d^{2}}{d x^{2}} x^{2} y^{2} \\
& -4 y^{\prime \prime} x^{2} y-2 \frac{d}{d x} x y^{2}-4 y^{\prime} x y+8 c y^{3} .
\end{aligned}
$$

In the case $c=0$ the linear differential operator is

$$
\mathcal{L}(x)=\frac{\partial \hat{f}_{7}^{(1)}\left(x, c_{0}\right)}{\partial y}=2 \frac{d^{2}}{d x^{2}} x^{2} c_{0}^{3}+2 \frac{d}{d x} x c_{0}^{3}-2 \frac{d^{2}}{d x^{2}} x^{2} c_{0}^{2}-2 \frac{d}{d x} x c_{0}^{2} .
$$


The characteristic polynomial

$$
\nu(k)=2 k^{2} c_{0}^{2}\left(c_{0}-1\right)
$$

has two roots which depend on $c_{0} \neq 0$. If $c_{0}=1$, then any number $k$ is a double root of the characteristic polynomial and $\mathcal{L}(x) \equiv 0$. For any $c_{0} \neq 1$ the polynomial (3.4.21) has the double root $k=0$.

The cone of the problem is $\mathcal{K}=\{\operatorname{Re} k>0$ or $\operatorname{Re} k=0, \operatorname{Im} k \neq 0\}$. If $c_{0} \neq 0,1$, then $k \notin \mathcal{K}$; i.e., there are no critical numbers and the expansion is in integral increasing powers of $x$. The expansion of the solution is

$$
\mathcal{B}_{10}: \quad y=c_{0}+\sum_{s=1}^{+\infty} c_{s} x^{s}
$$

where the complex coefficient $c_{0} \neq 0,1$ is arbitrary, and the remaining complex coefficients $c_{s}$ are uniquely determined. Expansion (3.4.22) was known before (see [54, $\S 46$, formula (46.4)]. By Theorem 1.7.2 the series (3.4.22) converges for sufficiently small $|x|$.

The second approximation to the solution of the equation is $y=c_{0}+c_{1} x$. The second approximation to equation (3.1.1) is

$$
\begin{aligned}
\hat{\hat{f}}_{7}(x, y)= & 2 y^{\prime \prime} x^{3} y-y^{\prime 2} x^{3}-2(b-d) x y^{2}+2 y^{\prime \prime} x^{3} y^{2}-2 y^{\prime 2} x^{3} y+6 y^{\prime} x^{2} y^{2} \\
& -4 x y^{3}(b-d)-4 y^{\prime \prime} x^{3} y^{3}+6 y^{\prime 2} x^{3} y^{2}-2 x y^{4}(b-d) .
\end{aligned}
$$

The coefficient $c_{1}$ is given by $c_{1}=-\frac{b_{1}}{\nu(1)}$, where

$$
b_{1}=x^{-1} \hat{\hat{f}}_{7}^{(1)}\left(x, c_{0}\right)=-2(b-d) c_{0}^{2}\left(c_{0}-1\right)^{2}, \quad \nu(1)=2 c_{0}^{2}\left(c_{0}-1\right),
$$

so that

$$
c_{1}=(b-d)\left(c_{0}-1\right) .
$$

If $c_{0}=1$, i.e., $\mathcal{L}(x) \equiv 0$, we make the substitution $y=1+u$ in equation (3.1.1) with $c=0$ and obtain the equation

$$
\begin{aligned}
g(x, u) \stackrel{\text { def }}{=} u^{\prime \prime} x^{2}(x-1)^{2}(u+1) u(u+1-x) \\
-u^{\prime 2}\left[x^{2}(x-1)^{2} u(u+1-x)\right. \\
\left.\quad+x^{2}(x-1)^{2}(u+1)^{2}(u+1-x)+x^{2}(x-1)^{2}(u+1) u\right] \\
+2 u^{\prime}\left[x(x-1)^{2}(u+1) u(u+1-x)\right. \\
\quad+x^{2}(x-1)(u+1) u(u+1-x)+x^{2}(x-1)^{2}(u+1) \\
\left.\quad-2 b x u^{2}(u+1-x)^{2}+2 d x(x-1)(u+1)^{2} u^{2}\right]=0 .
\end{aligned}
$$

Equation (3.4.25) has the trivial solution $u=0$. The corresponding exceptional solution of equation (3.1.1) is $\mathcal{I}_{2}: y=1$.

The cone of the problem is $\mathcal{K}=\left\{p_{1} \leq 0, p_{2}<0\right\}$. The support $\mathbf{S}(g)$ of the left-hand side of equation (3.4.25), its convex hull $\Gamma(g)$, and the faces (vertices $G_{i}^{(0)}$ and edges $G_{i}^{(1)}$, $i=1, \ldots, 5)$ are shown in Figure $18(a)$. The corresponding real normal cones $\mathbf{U}_{i}^{(j)}$ are shown in Figure 18 (b).

Normal cones that have nonempty intersection with the cones of the problem $\mathcal{K}$ are $\mathbf{U}_{1}^{(0)}$ and $\mathbf{U}_{1}^{(1)}$.

The reduced equation corresponding to the vertex $G_{1}^{(0)}$ is

$$
\hat{g}_{1}^{(0)}(x, u) \stackrel{\text { def }}{=} 2 u^{\prime \prime} u x^{2}-u^{\prime 2} x^{2}+2 u^{\prime} u x=0
$$



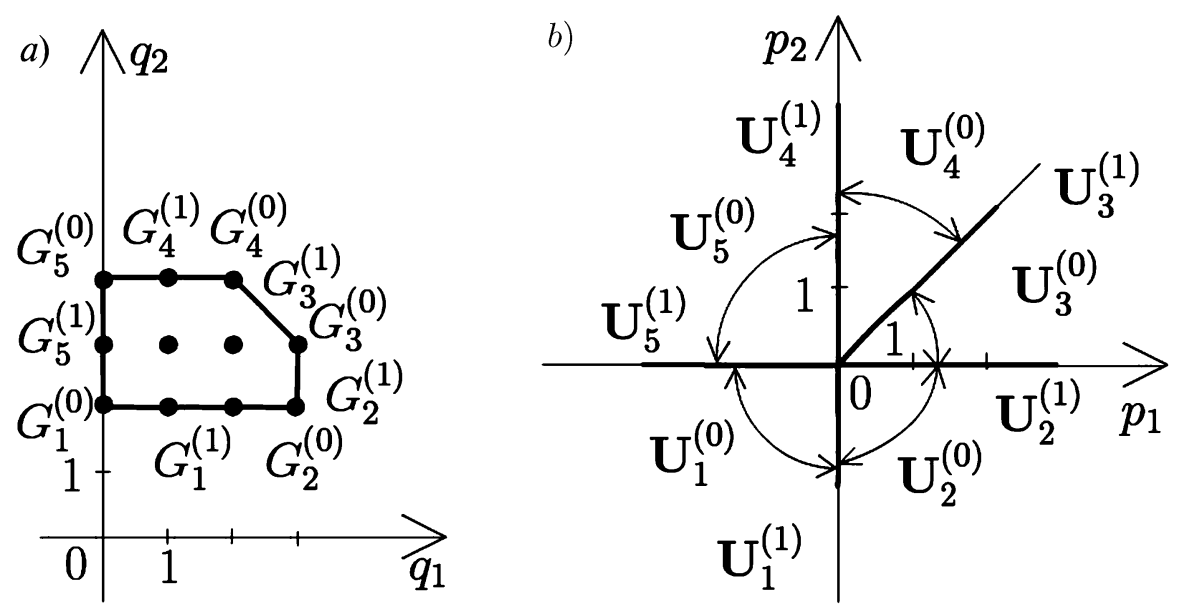

Figure 18. For equation (3.4.25): (a) the support, the polygon, and its faces; (b) normal cones of faces.

The first approximation to a solution is $u=c_{r} x^{r}$, where $c_{r}$ is an arbitrary nonzero constant. The exponent $r$ is determined from the characteristic equation

$$
\chi(r)=r^{2}=0,
$$

i.e., $r_{1,2}=0$. Since the vector $P=\omega(1, r)=-(1,0)$ is not in $\mathbf{U}_{1}^{(0)} \cap \mathcal{K}$, there are no appropriate power solutions corresponding to the vertex $G_{1}^{(0)}$. A simple analysis shows that all solutions of equation (3.4.26) are of the form $u=C_{1} \ln ^{2}\left(C_{0} x\right)$, where $C_{0}$ and $C_{1}$ are arbitrary constants. All these solutions have order $p=0$; however, $|u| \rightarrow \infty$ as $x \rightarrow 0$, so these solutions should be ignored.

The reduced equation corresponding to the edge $G_{1}^{(1)}$ is

$$
\begin{aligned}
\hat{g}_{1}^{(1)}(x, u) \stackrel{\text { def }}{=} & 2 u^{\prime \prime} u x^{2}-{u^{\prime}}^{2} x^{2}+2 u^{\prime} u x-6 u^{\prime \prime} u x^{3}+3{u^{\prime}}^{2} x^{3}-6 u^{\prime} u x^{2} \\
& -2(b-d) u^{2} x+6 u^{\prime \prime} u x^{4}-30{u^{\prime}}^{2} x^{4}+6 u^{\prime} u x^{3} \\
& -2(d+2 b) u^{2} x^{2}-2 u^{\prime \prime} u x^{5}+{u^{\prime}}^{2} x^{5}-2 u^{\prime} u x^{4}-2 b u^{2} x^{3}=0 .
\end{aligned}
$$

This edge is horizontal. The reduced equation corresponding to its left vertex $G_{1}^{(0)}=$ $(0,2)$ is (3.4.26). For both equations (3.4.26) and (3.4.28) the total differentiation order is $\Delta\left(\hat{g}_{1}^{(1)}\right)=\Delta\left(\hat{g}_{1}^{(0)}\right)=2$. By Theorem 1.3.4, equation (3.4.28) does not have nonpower solutions $u \rightarrow 0$ as $x \rightarrow 0$.

Let us compute nonpower solutions of equation (3.4.1). In our case, the determining equation (3.4.7) has an arbitrary solution. The cone of the problem is $\mathcal{K}=\left\{p_{1} \geq 0\right\}$, and moreover, $y \neq$ const.

For $c=0$ the support $\mathbf{S}(\varphi)$ of equation (3.4.8) consists of two points $Q_{1}$ and $Q_{3}$. The convex hull of the support is the interval coinciding with the edge $\Phi_{3}^{(1)}$ (Figure 17 $(a)$ ). Normal cones are $\mathbf{U}_{1}^{(0)}=\left\{p_{2}<0\right\}, \mathbf{U}_{3}^{(0)}=\left\{p_{2}>0\right\}, \mathbf{U}_{3}^{(1)}=\{\lambda(1,0), \lambda>0\}$. Each of them has a nonempty intersection with the support of the problem.

The vertex $\Phi_{1}^{(0)}$ was considered in the case $c \neq 0$ (see Subsection 4.2 in Chapter 3, equation (3.4.9) $)$. The vector $P=(1,0)$ is in the cone of the problem $\mathcal{K}$. However, the corresponding solution is constant, which is not suitable. Therefore, there are no solutions corresponding to the vertex $\Phi_{1}^{(0)}$. 
The reduced equation corresponding to the vertex $\Phi_{3}^{(0)}$ is

$$
\hat{\varphi}_{3}^{(0)}(\xi, y) \stackrel{\text { def }}{=} 2 \ddot{y} y^{3}-3 \dot{y}^{2} y^{2}=0 .
$$

The normal cone is $\mathbf{U}_{3}^{(0)}=\left\{p_{2}>0\right\}$. We are looking for solutions of this equation in the form $y=c_{r} \xi^{r}$, where $c_{r} \neq 0$ is an arbitrary constant. Let us compute the exponent $r$. The characteristic equation

$$
\chi(r) \stackrel{\text { def }}{=} \xi^{-4 r+2} \hat{\varphi}_{3}^{(0)}\left(\xi, \xi^{r}\right) \stackrel{\text { def }}{=}-r^{2}-2 r=0
$$

has the roots $r_{1}=0$ and $r_{2}=-2$. The solution corresponding to the vector $P_{1}=$ $\omega\left(1, r_{1}\right)=(1,0)$ is constant, so not suitable. On the other hand, the vector $P_{2}=$ $\omega\left(1, r_{2}\right)=(1,-2)$ is not in the normal cone $\mathbf{U}_{3}^{(0)}$. Therefore, there are no solutions corresponding to the vertex $\Phi_{3}^{(0)}$.

The reduced equation corresponding to the vertical edge $\Phi_{3}^{(1)}$ is

$$
\hat{\varphi}_{3}^{(1)}(\xi, y) \stackrel{\text { def }}{=} 2 \ddot{y} y^{3}-3 \dot{y}^{2} y^{2}-2 \ddot{y} y^{2}+2 \dot{y}^{2} y=0 .
$$

The root of the determining equation can be any constant. But the solution $y(\xi)=$ const is not suitable.

According to Subsection 3.2 of Chapter 1 we perform one more logarithmic transformation $\eta=\ln \xi$. We obtain

$$
\dot{y}=\frac{1}{\xi} \frac{d y}{d \eta}, \quad \ddot{y}=\frac{1}{\xi^{2}}\left(\frac{d^{2} y}{d \eta^{2}}-\frac{d y}{d \eta}\right) .
$$

Equation (3.4.29) takes the form

$$
\hat{\varphi}_{3}^{(1)}(\xi, y) \stackrel{\text { def }}{=} \sigma(\eta, y) \stackrel{\text { def }}{=}-2\left(\frac{d^{2} y}{d \eta^{2}}-\frac{d y}{d \eta}\right) y^{2}(1-y)+\left(\frac{d y}{d \eta}\right)^{2} y(2-3 y)=0 .
$$

The support $\mathbf{S}(\sigma)$ consists of four points $Q_{1}=(-1,3), Q_{2}=(-1,4), Q_{3}=(-2,4)$, $Q_{4}=(-2,3)$. The convex hull of these points is the square with vertices at these points. The boundary of this square consists of four vertices and four edges. The normal cones are quadrants and coordinate planes $p_{1}, p_{2}$.

The cone of the problem is $\mathcal{K}=\left\{p_{1} \geq 0\right\}$ and the solution is $y(\eta) \neq$ const. The normal cones that have a nonempty intersection with the cone of the problem are those of the left, top, and bottom edges, and of two right-side vertices.

The top and the bottom edges correspond to the vertices $\Phi_{3}^{(0)}$ and $\Phi_{1}^{(0)}$, for which the absence of suitable solutions has been established earlier. The reduced equation corresponding to the right edge is

$$
2 \frac{d y}{d \eta} y^{2}(1-y)=0 .
$$

It has constant solutions only, which is not suitable. The reduced equations corresponding to the two right-side vertices are parts of equation (3.4.30). Therefore, the corresponding solutions are constant as well, and such solutions are not suitable. Therefore, there are no solutions corresponding to the edge $\Phi_{3}^{(1)}$.

So, in the case $c=0$ there are no nonpower solutions of equation (3.4.1).

4.4. Explicit form of solutions of (3.4.1). It is clear that equation 3.4.1 and equation (2.3.1) for $a=0$ are the same equation. Therefore, we use results of Chapter 2 and consider equation (2.3.33).

In the case $a=0$ equation (2.3.33) takes the form

$$
\frac{d y}{d \xi}= \pm y \sqrt{2 c-C_{2}+C_{2} y} \text {. }
$$


Integration of equation (3.4.31) depends on $C_{2}$ and on $c$. Consider three cases depending on the value of $C_{2}$.

Case $C_{2}=2 c$. If $a=0, c \neq 0$, then equation (2.3.33) has a solution (2.3.36) coming from the case $a \neq c \neq 0$. The corresponding expansion is (2.3.37).

In the case $a=c=0$, equation (3.4.31) becomes $\frac{d y}{d \xi}=0$. It has a solution $y=C_{3}$, where $C_{3}$ is an arbitrary constant. The corresponding family of expansions of solutions of the complete equation is (3.4.22).

Case $C_{2}=0$. Equation (3.4.31) takes the form

$$
\frac{d y}{d \xi}= \pm y \sqrt{2 c}
$$

If $a=0, c \neq 0$, then equation (3.4.32) has a solution

$$
y=C_{4} x^{ \pm \sqrt{2 c}},
$$

where $C_{4}$ is an arbitrary constant.

The function (3.4.33) is a solution of the reduced equation (3.4.1) corresponding to the edge $\Gamma_{3}^{(1)}$. Furthermore, this function is a solution of the reduced equation corresponding to each of the vertices $Q_{4}=(0,3)$ and $Q_{6}=(0,4)$ (Figure 14 $(b)$ ).

For $\operatorname{Re} \sqrt{2 c}=0$ we obtain the initial parts (3.4.33) of expansions (3.4.6) from families $\mathcal{B}_{8}$ with $\operatorname{Im} \sqrt{2 c}>0$ and $\mathcal{B}_{9}$ with $\operatorname{Im} \sqrt{2 c}<0$.

Case $0 \neq C_{2} \neq 2 c$. Let

$$
t^{2}=2 c-C_{2}+C_{2} y
$$

Then equation (3.4.31) takes the form

$$
\frac{d t}{d \xi}= \pm \frac{\left(t^{2}-2 c+C_{2}\right)}{2}
$$

Equation (3.4.35) has the integral

$$
\ln \frac{t-\sqrt{2 c-C_{2}}}{t+\sqrt{2 c-C_{2}}}= \pm \sqrt{2 c-C_{2}}\left(\xi+C_{16}\right),
$$

where $C_{16}$ is an arbitrary constant. Hence

$$
\frac{t-\sqrt{2 c-C_{2}}}{t+\sqrt{2 c-C_{2}}}=\exp \left[ \pm \sqrt{2 c-C_{2}}\left(\xi+C_{16}\right)\right] \stackrel{\text { def }}{=} \sigma .
$$

Taking into account (3.4.34) and (3.4.36) we obtain

$$
y=\frac{2 c-C_{2}}{C_{2}} \frac{4 \sigma}{(1-\sigma)^{2}} .
$$

The right-hand side of (3.4.37) can be written in the form

$$
y=\frac{2 c-C_{2}}{C_{2}}\left(\frac{2}{1 / \sqrt{\sigma}-\sqrt{\sigma}}\right)^{2} .
$$

Set $2 i \eta=\ln \sigma \stackrel{\text { def }}{=} \pm \sqrt{2 c-C_{2}}\left(\xi+C_{16}\right)$. Taking into account that $\xi=\ln x$ we have

$$
\eta= \pm i \frac{\sqrt{2 c-C_{2}}}{2}\left(\ln x+C_{16}\right) .
$$

Then equation (3.4.38) takes the form

$$
y=\frac{C_{2}-2 c}{C_{2}} \frac{1}{\sin ^{2} \eta} .
$$


According to (3.4.36),$y$ can be expanded in a series in powers of $\sigma=\left(C_{17} x\right)^{ \pm \sqrt{2 c-C_{2}}}$, where $C_{17}$ is an arbitrary constant.

If $2 c-C_{2}$ is a negative real number, then the number $i \sqrt{2 c-C_{2}} \stackrel{\text { def }}{=} 2 \gamma$ is real. In this case $\eta=\gamma \ln \left(C_{17} x\right)$. Formula (3.4.39) shows that $y$ can be expanded in a series in integral powers of $\sigma$, i.e., in integral powers of $x^{2 \gamma i}$, which yields a series in purely imaginary powers of $x$. For $a=0$ this series corresponds to families $\mathcal{B}_{7}^{\tau}$ of exotic expansions (3.4.3) with $\operatorname{Re} \rho=0$ and $\rho^{2} \neq 2 c$. According to (3.4.38) the inverse expansion $y^{-1}$ does not depend on the sign of $\gamma$, i.e., of $\tau$. By Theorem 1.5.3, expansions $y^{-1}$ inverse to (3.4.3) also do not depend on $\tau$.

Hence, we have proved the following result.

Theorem 3.4.1. (a) For $x \rightarrow 0$ and $a=0$ there exist two two-parameter families of exotic expansions of solutions with constant complex coefficients:

$$
\mathcal{B}_{7}^{\tau}: \quad y=\frac{\rho^{2}}{2 c-\rho^{2}} \frac{1}{\sin ^{2}\left[\ln \left(C_{11} x\right)^{\gamma}\right]}+\sum_{\operatorname{Re} s \geq 1} c_{s} x^{s} .
$$

Here $\rho$ is an arbitrary purely imaginary constant, $\rho^{2} \neq 2 c, \tau=\operatorname{sgn}(\operatorname{Im} \rho), 2 \gamma=i \rho$, $s \in\{\rho+l \rho+m(1-\rho), l, m \geq 0 ; l+m>0 ; l, m \in \mathbb{Z}\}, C_{11}$ is an arbitrary nonzero constant, and the $c_{s}$ are uniquely determined constants. The families of inverse expansions $y^{-1}$ to families $\mathcal{B}_{7}^{+}$and $\mathcal{B}_{7}^{-}$coincide.

(b) For $x \rightarrow 0$ and $a=0, c \neq 0$ there exist two one-parameter families of power expansions of solutions

$$
\mathcal{B}_{8,9}: \quad y=c_{\rho} x^{\rho}+\sum_{\operatorname{Re} s \geq 1} c_{s} x^{s},
$$

where $\rho$ is a purely imaginary constant, $\rho^{2}=2 c, \operatorname{Im} \rho>0$ for the family $\mathcal{B}_{8}$ and $\operatorname{Im} \rho<0$ for the family $\mathcal{B}_{9}, s \in\{\rho+l(1-\rho)+m, l, m \geq 0 ; l+m>0 ; l, m \in \mathbb{Z}\}$, and the complex coefficients are as follows: $c_{\rho}$ is an arbitrary nonzero constant and the $c_{s}$ are uniquely determined constants.

(c) For $x \rightarrow 0$ and $a=c=0$ there exists a one-parameter family of power expansions of solutions with constant complex coefficients

$$
\mathcal{B}_{10}: \quad y=c_{0}+\sum_{s=1}^{+\infty} c_{s} x^{s},
$$

where $c_{0} \neq 0,1$ is an arbitrary constant and the $c_{s}$ are uniquely determined constants .

\section{EXPANSIONS NEAR INFINITY FOR $a=0, b \neq 0$}

5.1. Expansions near infinity corresponding to the vertex $\Gamma_{2}^{(0)}$. The transformation

$$
x=\frac{1}{x^{*}}, \quad y=\frac{y^{*}}{x^{*}},
$$

which is the superposition of symmetries (2.1.6) and (2.1.8), sends the vertex $\Gamma_{5}^{(0)}=(2,4)$ to the vertex $\Gamma_{6}^{(0)}=(0,4)$. Therefore the expansions corresponding to the vertex $\Gamma_{5}^{(0)}$ are obtained from those corresponding to the vertex $\Gamma_{6}^{(0)}$ by applying (3.5.1). In this way, we obtain the family

$$
\mathcal{C}_{\infty}^{\infty}: \quad y=c_{r} x^{r}+\sum_{s} c_{s} x^{s}
$$


A. D. BRUNO AND I. V. GORYUCHKINA

where $r=1+\sqrt{1-2 d}, \operatorname{Re} \sqrt{1-2 d}>0, s \in\{r+l(1-r)-m ; l, m \geq 0 ; l+m>0$; $l, m \in \mathbb{Z}\}$, and the complex coefficients are as follows: $c_{r}$ is an arbitrary constant, and the $c_{s}$ are uniquely determined constants.

For $\operatorname{Re} r>2$ the second approximation to the solution is $y=c_{r} x^{r}+c_{r-1} x^{r-1}$, where $c_{r-1}=c_{r} \frac{(c+b+d-r)}{r}$.

For $1<\operatorname{Re} r \leq 2$ the second approximation to the solution is $y=c_{r} x^{r}+c_{1} x$, where $c_{1}=0$.

5.2. Expansions near infinity corresponding to the edge $\Gamma_{5}^{(1)}$. For $a=0, b \neq 0$, $d=\frac{1}{2}$ we have the following family of power expansions $\mathcal{G}_{10}$ corresponding to the edge $\Gamma_{5}^{(1)}$ and obtained by the symmetry (3.5.1) from the family of expansions $\mathcal{B}_{10}$ corresponding to the edge $\Gamma_{7}^{(1)}$ :

$$
\mathcal{G}_{10}: \quad y=c_{1} x+\sum_{s=0}^{+\infty} c_{-s} x^{-s},
$$

with the following complex coefficients: $c_{1} \neq 0,1$ is an arbitrary constant, and the $c_{-s}$ are uniquely determined constants. The second approximation to the solution is given by $y=c_{1} x+c_{0}$, where

$$
c_{0}=\left(c_{1}-1\right)(1-2 c-2 b) .
$$

For $a=0, d \neq \frac{1}{2}$ we have the following family of complicated expansions corresponding to the edge $\Gamma_{7}^{(1)}$ :

$$
\mathcal{G}_{3}: \quad y=\varphi_{1} x+\sum_{\sigma=0}^{\infty} \varphi_{-\sigma} x^{-\sigma}
$$

where

$$
\varphi_{1}=\frac{4}{1-2 d} \frac{1}{\ln ^{2} x}+c_{-3} \frac{1}{\ln ^{3} x}+\sum_{s=4}^{+\infty} c_{-s}\left(\frac{1}{\ln x}\right)^{s}
$$

and the complex coefficients are as follows: $c_{-3}$ is arbitrary, and the remaining $c_{-s}$ are uniquely determined. Also, $\varphi_{-\sigma}$ are series in decreasing powers of simple logarithms.

For $a=0, d \neq \frac{1}{2}$ there exist two families of exotic expansions

$$
\mathcal{G}_{7}^{\tau}: \quad y=c_{\rho} x^{\rho}+\sum_{s} c_{s} x^{s},
$$

where $\tau=\operatorname{sgn}(\rho-1), \rho$ is an arbitrary complex number such that $\operatorname{Re} \rho=1, \operatorname{Im} \rho \neq 0$, $\rho-1 \neq \sqrt{1-2 d}, s \in\{\rho+l(\rho-1)-m \rho ; l, m \geq 0 ; l+m>0 ; l, m \in \mathbb{Z}\}$. Complex coefficients are as follows: $c_{\rho}$ is an arbitrary nonzero constant, and the $c_{s}$ are uniquely determined constants.

For $a=0, d>\frac{1}{2}$ we have two families of expansions with $\rho=1+\sqrt{1-2 d}$, namely, the two families of exotic expansions

$$
\mathcal{G}_{8,9}: \quad y=c_{\rho} x^{\rho}+\sum_{\operatorname{Re} s \leq 0} c_{s} x^{s},
$$

where $\operatorname{Re} \rho=1, \operatorname{Im} \rho \neq 0, \operatorname{Im} \rho>0$ for the family $\mathcal{G}_{8}$ and $\operatorname{Im} \rho<0$ for the family $\mathcal{G}_{9}, s \in\{\rho-l \rho-m \rho ; l, m \geq 0 ; l+m>0 ; l, m \in \mathbb{Z}\}$, and the complex coefficients are as follows: $c_{\rho}$ is an arbitrary nonzero constant, and the $c_{s}$ are uniquely determined constants. 
6. Summary of Results in the Case $a=0, b \neq 0$

Theorem 3.6.1. In the case $a=0, b \neq 0$, equation (2.1.1) with $x \rightarrow 0$ has 21 families of expansions of solutions. Of these, 15 families $\mathcal{A}_{0}, \mathcal{H}_{i}, i=1, \ldots, 6, \mathcal{H}_{0}, \mathcal{H}_{1}^{\tau}, \mathcal{H}_{2}^{\tau}, \mathcal{H}_{6}^{\tau}$, $\tau= \pm 1$, and $\mathcal{B}_{3}$ corresponding to the vertex $\Gamma_{4}^{(0)}$, the edge $\Gamma_{1}^{(1)}$, and the edge $\Gamma_{4}^{(1)}$ come from the case $a \cdot b \neq 0$. The remaining 6 families are new:

the family $\mathcal{C}_{0}^{\infty}$, which exists for $c \neq 0$, is given by formulas (3.2.8), (3.2.7) and has one parameter;

the families $\mathcal{B}_{8,9}$, which exist for $c<0$, are given by formula (3.4.6), and have one parameter;

the family $\mathcal{B}_{10}$, which exists for $c=0$, is given by formula (3.4.22), and has one parameter;

the family $\mathcal{B}_{7}^{\tau}$, which exists for $c \neq 0$, is given by formula (3.4.3) with first approximation (3.4.39), and has two parameters.

Remark 3.6.1. The families $\mathcal{C}_{0}^{\infty}, \mathcal{B}_{8}-\mathcal{B}_{10}, \mathcal{B}_{7}^{\tau}$ are also called basic. Applying the symmetries (2.1.6), (2.1.8), and (2.1.9) of equation P6 we obtain the remaining families of expansions.

The one-parameter families $\mathcal{C}_{0}^{\infty}, \mathcal{B}_{8}-\mathcal{B}_{10}$ are power families, $\mathcal{B}_{7}^{\tau}$ are exotic, and $\mathcal{B}_{3}$ is complicated. The existence of basic families $\mathcal{B}_{3}, \mathcal{C}_{0}^{\infty}, \mathcal{B}_{8}-\mathcal{B}_{10}, \mathcal{B}_{7}^{\tau}$ for various values of the parameters with $a=0$ is shown in the last two columns of Table 1 on page 9 .

Theorem 3.6.2. For $a=0, b \neq 0$, equation (2.1.1) has 21 families of expansions of solutions as $x \rightarrow \infty$. Of these families, 15 families $\mathcal{A}_{\infty}, \mathcal{D}_{i}, i=1, \ldots, 6, \mathcal{D}_{0}, \mathcal{D}_{1}^{\tau}, \mathcal{D}_{2}^{\tau}$, $\mathcal{D}_{6}^{\tau}, \tau= \pm 1$, and $\mathcal{G}_{3}$ corresponding to the vertex $\Gamma_{2}^{(0)}$, the edge $\Gamma_{2}^{(1)}$, and the edge $\Gamma_{3}^{(1)}$ come from the case $a \cdot b \neq 0$. The remaining 6 families are new:

the family $\mathcal{C}_{\infty}^{\infty}$, which exists for $d \neq \frac{1}{2}$, is defined by formula (3.5.2), and has one parameter;

the families $\mathcal{G}_{8,9}$, which exist for $d>\frac{1}{2}$, are defined by formula (3.5.8), and have one parameter;

the family $\mathcal{G}_{10}$, which exists for $d=\frac{1}{2}$, is defined by formula (3.5.3), and has one parameter;

the family $\mathcal{G}_{7}^{\tau}$, which exists for $d \neq \frac{1}{2}$, is defined by formula (3.5.7), and has two parameters.

The sixth Painlevé equation has three symmetries, (2.1.6), (2.1.8), (2.1.9). Combining the first two, we obtain the symmetry (3.5.1):

$$
(x, y, a, b, c, d) \rightarrow\left(\frac{1}{x^{*}}, \frac{y^{*}}{x^{*}}, a^{*}, b^{*},-d^{*}+\frac{1}{2},-c^{*}+\frac{1}{2}\right) .
$$

This symmetry sends families of expansions for $x \rightarrow 0$ to families of expansions for $x \rightarrow$ $\infty$, with the families $\mathcal{C}_{0}^{\infty}, \mathcal{B}_{8}-\mathcal{B}_{10}, \mathcal{B}_{7}^{\tau}$ becoming the families $\mathcal{C}_{\infty}^{\infty}, \mathcal{G}_{8}-\mathcal{G}_{10}, \mathcal{G}_{7}^{\tau}$ respectively (and vice versa). Let us illustrate this by using as an example the families $\mathcal{C}_{0}^{\infty}$ and $\mathcal{C}_{\infty}^{\infty}$.

Applying (3.6.1) to formula (3.2.8) we obtain

$$
\begin{aligned}
\frac{y^{*}}{x^{*}} & =c_{r} x^{*-r}+\sum_{s} c_{s} x^{*-s}, \\
y^{*} & =c_{r} x^{*-r+1}+\sum_{s} c_{s} x^{*-s+1} .
\end{aligned}
$$


Denote $c_{r^{*}}^{*}=c_{r}, r^{*}=-r+1, c_{s^{*}}^{*}=c_{s}, s^{*}=-s+1$, where $s^{*}$ runs over the set $\mathbf{K}^{*}=\left\{s^{*}=r^{*}+l\left(1-r^{*}\right)-m ; l, m>0 ; l+m \geq 0 ; l, m \in \mathbb{Z}\right\}$. We obtain the series

$$
y^{*}=c_{r^{*}}^{*} x^{* r^{*}}+\sum_{s^{*}} c_{s^{*}}^{*} x^{* s^{*}} .
$$

From Section 2 in Chapter 3 we know that in the case $1>|\operatorname{Re} \sqrt{2 c}|>0$ the second approximation to the solution corresponding to the vertex $\Gamma_{6}^{(0)}$ is $y=c_{r} x^{r}+c_{0}$, where $c_{r} \neq 0$ is an arbitrary constant and $r=-\sqrt{2 c}$. Using (3.6.1) we obtain the second approximation to the solution corresponding to the vertex $\Gamma_{5}^{(0)}$ for $1>\left|\operatorname{Re} \sqrt{1-2 d^{*}}\right|>0$,

$$
y^{*}=c_{r^{*}}^{*} x^{* r^{*}}+c_{1}^{*} x^{*},
$$

where $r^{*}=1+\sqrt{1-2 d^{*}}, c_{r^{*}}^{*} \neq 0, c_{r^{*}}^{*}$ is an arbitrary constant, and $c_{1}^{*}=0$.

Similarly, in the case $\left|\operatorname{Re} \sqrt{1-2 d^{*}}\right| \geq 1$ we obtain

$$
y^{*}=c_{r^{*}}^{*} x^{* r^{*}}+c_{r^{*}-1}^{*} x^{* r^{*}-1},
$$

where $r^{*}=1+\sqrt{1-2 d^{*}}, \quad c_{r^{*}}^{*} \neq 0, c_{r^{*}}^{*}$ is an arbitrary constant, and

$$
c_{r^{*}-1}^{*}=c_{r^{*}}^{*} \frac{\left(-r^{* 2}+r^{*}+c^{*}+b^{*}-d^{*}\right)}{r^{*}} .
$$

Finally, we remove the asterisks and get

$$
\mathcal{C}_{\infty}^{\infty}: \quad y=c_{r} x^{r}+\sum_{s} c_{s} x^{s}
$$

where the complex exponent $r$ is given by $r=1+\sqrt{1-2 d}, \operatorname{Re} \sqrt{1-2 d}>0, c_{r} \neq 0, c_{r}$ is an arbitrary complex constant, $c_{s}$ are uniquely determined constants, and $s \in \mathbb{C}$ runs over the set $\{r+l(1-r)-m ; l, m>0 ; l+m \geq 0 ; l, m \in \mathbb{Z}\}$; in the case $|\operatorname{Re} \sqrt{1-2 d}|$ $\geq 1$ the second approximation to expansion (3.6.3) is $y=c_{r} x^{r}+c_{r-1} x^{r-1}$, where $c_{r-1}=c_{r} \frac{\left(-r^{2}+r+c+b-d\right)}{r}$, and in the case $1>|\operatorname{Re} \sqrt{1-2 d}|>0$ the second approximation is $y=c_{r} x^{r}+c_{1} x$, where $c_{1}=0$.

\section{EXPansions in the CASE $a \neq 0, b=0$}

In this case, the polygon of equation P6 is shown in Figure 15) $(a)$ and the normal cones of generalized faces of this polygon are shown in Figure $15(b)$.

Using symmetry (2.1.6) one can obtain expansions in the case $b=0$ from expansions in the case $a=0$.

7.1. Expansions corresponding to the vertex $\Gamma_{8}^{(0)}=(3,2)$. This vertex is symmetric to the vertex $\Gamma_{6}^{(0)}$. Therefore expansions of solutions corresponding to the vertex $\Gamma_{8}^{(0)}$ are symmetric to expansions of solutions corresponding to the vertex $\Gamma_{6}^{(0)}$. Let us consider expansion (3.2.8). The symmetry (2.1.6) sends formula (3.2.8) to the following formula:

$$
\begin{aligned}
\frac{1}{y^{*}} & =c_{r} x^{*-r}+\sum_{s} c_{s} x^{*-s}, \\
y^{*} & =\frac{1}{\frac{c_{r}}{x^{* r}}\left(1+\sum_{s} \frac{c_{s}}{c_{r}} x^{*-s+r}\right)} .
\end{aligned}
$$

Since $\left|x^{*}\right| \rightarrow \infty$, expression (3.7.1) can be expanded in a formal series

$$
y^{*}=\frac{x^{* r}}{c_{r}} \sum_{n=0}^{+\infty}(-1)^{n}\left(\sum_{s} \frac{c_{s}}{c_{r}} x^{*-s+r}\right)^{n} .
$$


The first two terms of this series are

$$
y^{*}=\frac{x^{* r}}{c_{r}}-\sum_{s} \frac{c_{s}}{c_{r}^{2}} x^{*-s+2 r}+\cdots
$$

Set $c_{r}^{*}=\frac{1}{c_{r}}, c_{s^{*}}^{*}=-\frac{c_{s}}{c_{r}^{2}}, s^{*}=-s+2 r$, where $s^{*}$ runs over the set $\{r+l r-m$; $l, m>0 ; l+m \geq 0 ; l, m \in \mathbb{Z}\}$. We obtain the series

$$
y^{*}=c_{r}^{*} x^{* r}+\sum_{s^{*}} c_{s^{*}}^{*} x^{* s^{*}}+\cdots .
$$

The second approximation to the solution in the case $\operatorname{Re} r \leq-1$ is $y^{*}=c_{r}^{*} x^{* r}+c_{r-1}^{*} x^{* r-1}$, $r=-\sqrt{2 c}$,

$$
c_{r-1}^{*}=-\frac{c_{r+1}}{c_{r}^{2}}=-c_{r}^{*} \frac{c^{*}+d^{*}+a^{*}-r}{r-1} .
$$

In the case $-1<\operatorname{Re} r<0$ the second approximation is

$$
y^{*}=c_{r}^{*} x^{* r}+c_{2 r}^{*} x^{* 2 r},
$$

where $c_{2 r}^{*}=0$. Finally, deleting asterisks we obtain

$$
\mathcal{C}_{\infty}^{0}: \quad y=c_{r} x^{r}+\sum_{s} c_{s} x^{s}
$$

where the complex exponent $r=\sqrt{2 c}$ satisfies the condition $\operatorname{Re} r<0$, and $s$ runs over the set $\{r+l r-m, l, m>0, l+m \geq 0, l, m \in \mathbb{Z}\}$. The complex coefficients are as follows: $c_{r}$ is an arbitrary nonzero constant, and all $c_{s}$ are uniquely determined constants.

In the case $\operatorname{Re} r<-1$ expansion of the solution is $y=c_{r} x^{r}+c_{r-1} x^{r-1}$, where

$$
c_{r-1}=-c_{r} \frac{c+d+a-r}{r-1},
$$

and in the case $-1<\operatorname{Re} r<0$ the second approximation to the solution of the equation is $y=c_{r} x^{r}+c_{2 r} x^{2 r}$, where $c_{2 r}=0$. By Theorem 1.7.2 the series (3.7.4) converges for sufficiently large $|x|$.

7.2. Expansions corresponding to the vertex $\Gamma_{7}^{(0)}=(1,2)$. We have the following one-parameter family of expansions:

$$
\mathcal{C}_{0}^{0}: \quad y=c_{r} x^{r}+\sum_{s} c_{s} x^{s},
$$

where $r=1+\sqrt{1-2 d}, \operatorname{Re} r>1, s \in\{r+l(r-1)+m ; l, m>0 ; l+m \geq 0 ; l, m \in \mathbb{Z}\}$ and the complex coefficients are as follows: $c_{r}$ is an arbitrary nonzero constant, and all $c_{s}$ are uniquely determined constants. In the case $\operatorname{Re} r \geq 2$ the second approximation to the solution is $y=c_{r} x^{r}+c_{2 r-1} x^{2 r-1}, c_{2 r-1}=c_{r} \frac{r-c+a-d}{r}$, and in the case $1<\operatorname{Re} r<2$ the second approximation is $y=c_{r} x^{r}+c_{2 r-1} x^{2 r-1}, c_{2 r-1}=0$. According to Theorem 1.7.2 the series (3.7.5) converges for sufficiently small $|x|$.

7.3. Expansions of solutions corresponding to the edge $\Gamma_{10}^{(1)}$. For $c=0$ we have the following one-parameter family of power expansions:

$$
\mathcal{D}_{10}: \quad y=c_{0}+\frac{c_{-1}}{x}+\sum_{s=2}^{+\infty} \frac{c_{-s}}{x^{s}},
$$

where the complex coefficients are as follows: $c_{0} \neq 0,1$ is an arbitrary constant, $c_{-1}=$ $(d+a)\left(1-c_{0}\right) c_{0}$, and all $c_{-s}$ are uniquely determined constants.

By Theorem 1.7.2 the series (3.7.6) converges for sufficiently large $|x|$. 
For $c \neq 0$ we have the following one-parameter family of complicated expansions:

$$
\mathcal{D}_{3}: \quad y=\varphi_{0}+\sum_{\sigma=1}^{\infty} \varphi_{-\sigma} x^{-\sigma}
$$

where

$$
\varphi_{0}=\frac{c}{2} \ln ^{2} x+c_{1} \ln x+\sum_{s=0}^{+\infty} c_{-s}\left(\frac{1}{\ln x}\right)^{s}+\cdots,
$$

and the complex coefficients are as follows: $c_{1}$ is an arbitrary constant, and all $c_{-s}$ are uniquely determined constants; $\varphi_{-\sigma}$ are series in decreasing powers of logarithms.

For $c \neq 0$ there exists the following family of semiexotic expansions:

$$
\mathcal{D}_{7}: \quad y=c_{\rho} x^{\rho}+c_{0}+c_{-\rho} x^{-\rho}+\sum_{s} c_{s} x^{s}
$$

where $\rho$ is a complex constant such that $\operatorname{Re} \rho=0, \operatorname{Im} \rho \neq 0, \rho^{2} \neq 2 c, s \in\{l+k \rho$; $l \leq-1 ;|k| \leq|l| ; l, k \in \mathbb{Z}\}$. The complex coefficients are as follows: $c_{\rho}$ is an arbitrary nonzero constant, and $c_{0}, c_{-} \rho$, and $c_{s}$ are uniquely determined constants.

For $c<0$ we have two families of expansions with $\rho=\sqrt{2 c}$ :

$$
\mathcal{D}_{8,9}: \quad y=c_{\rho} x^{\rho}+\sum_{\operatorname{Re} s \leq-1} c_{s} x^{s},
$$

where $\operatorname{Re} \rho=0, \operatorname{Im} \rho \neq 0, \operatorname{Im} \rho<0$ for the family $\mathcal{D}_{8}$ and $\operatorname{Im} \rho>0$ for the family $\mathcal{D}_{9}$, $s \in\{\rho-l \rho-m ; l, m \geq 0 ; l+m>0 ; l, m \in \mathbb{Z}\}$. The complex coefficients are as follows: $c_{\rho}$ is an arbitrary nonzero constant, and the $c_{s}$ are uniquely determined constants.

7.4. Expansions of solutions corresponding to $\Gamma_{8}^{(1)}$. For $d=\frac{1}{2}$ we have the following one-parameter family of power expansions:

$$
\mathcal{H}_{10}: \quad y=c_{1} x+\sum_{s=2}^{+\infty} c_{s} x^{s}
$$

where the complex coefficients are as follows: $c_{1} \neq 0,1$ is an arbitrary constant, $c_{2}=$ $-c_{1} \times\left(1-c_{1}\right)(1-2 c+2 a)$, and all $c_{s}$ are uniquely determined constants.

This series converges for sufficiently small $|x|$.

For $d \neq \frac{1}{2}$ we have the following one-parameter family of complicated expansions:

$$
\mathcal{H}_{3}: \quad y=\varphi_{1} x+\sum_{\sigma=2}^{\infty} \varphi_{\sigma} x^{\sigma}
$$

where

$$
\varphi_{1}=\frac{1-2 d}{4} \ln ^{2} x+c_{1} \ln x+\sum_{s=0}^{+\infty} c_{-s}(\ln x)^{-s},
$$

and the complex coefficients are as follows: $c_{1}$ is an arbitrary constant, and all $c_{-s}$ are uniquely determined constants; $\varphi_{\sigma}$ are the series in decreasing powers of logarithms.

For $d \neq \frac{1}{2}$ there exists one family of semiexotic expansions,

$$
\mathcal{H}_{7}: \quad y=c_{\rho} x^{\rho}+c_{1} x+c_{2-\rho} x^{2-\rho}+\sum_{s} c_{s} x^{s}
$$

where the complex number $\rho$ satisfies the conditions $\operatorname{Re} \rho=1, \operatorname{Im} \rho \neq 0, \rho-1 \neq \sqrt{1-2 d}$, $s \in\{l+k(\rho-1) ; l \geq 2 ;|k| \leq l ; l, k \in \mathbb{Z}\}$. The complex coefficients are as follows: $c_{\rho}$ is an arbitrary complex constant, and $c_{1}, c_{2-\rho}$, and $c_{s}$ are uniquely determined constants. 
For $a=0, d>\frac{1}{2}$ we have two families of expansions,

$$
\mathcal{H}_{8,9}: \quad y=c_{\rho} x^{\rho}+\sum_{\operatorname{Re} s \geq 2} c_{s} x^{s}
$$

where $\operatorname{Re} \rho=1, \operatorname{Im} \rho \neq 0, \operatorname{Im} \rho<0$ for the family $\mathcal{H}_{8}$ and $\operatorname{Im} \rho>0$ for the family $\mathcal{H}_{9}$, $s \in\{\rho+l \rho+m ; m \geq 0 ; l \geq 1 ; l, m \in \mathbb{Z}\}$, and the complex coefficients are as follows: $c_{\rho}$ is an arbitrary nonzero constant, and the $c_{s}$ are uniquely determined constants.

\subsection{Summary of results in the case $a \neq 0, b=0$.}

Theorem 3.7.1. In the case $a \neq 0, b=0$, equation (2.1.1) has 21 families of expansions of solutions as $x \rightarrow 0$. Of these, 16 families $\mathcal{A}_{0}, \mathcal{B}_{i}, i=1, \ldots, 6, \mathcal{B}_{0}^{\tau}, \mathcal{B}_{1}^{\tau}, \mathcal{B}_{2}^{\tau}, \mathcal{B}_{6}^{\tau}, \tau= \pm 1$, and $\mathcal{H}_{3}$ corresponding to the vertex $\Gamma_{4}^{(0)}$, to the edge $\Gamma_{4}^{(1)}$, and to the edge $\Gamma_{1}^{(1)}$ come from the case $a \cdot b \neq 0$. The remaining 5 families of expansions of solutions are new:

$\mathcal{C}_{0}^{0}$, which exists for $d \neq \frac{1}{2}$, is given by formula (3.7.5), and has one parameter;

$\mathcal{H}_{8,9}$, which exist for $d>\frac{1}{2}$, are given by formula (3.7.15), and have one parameter;

$\mathcal{H}_{10}$, which exists for $d=\frac{1}{2}$, is given by formula (3.7.11), and has one parameter; $\mathcal{H}_{7}$, which exists for $d \neq \frac{1}{2}$, is given by formula (3.7.14), and has two parameters.

Theorem 3.7.2. In the case $a \neq 0, b=0$, equation (2.1.1) has 21 families of expansions of solutions as $x \rightarrow \infty$. Of these expansions, 16 families $\mathcal{A}_{\infty}, \mathcal{G}_{i}, i=1, \ldots, 6, \mathcal{G}_{0}^{\tau}, \mathcal{G}_{1}^{\tau}$, $\mathcal{G}_{2}^{\tau}, \mathcal{G}_{6}^{\tau}, \tau= \pm 1$, and $\mathcal{D}_{3}$, corresponding to the vertex $\Gamma_{2}^{(0)}$, the edge $\Gamma_{3}^{(1)}$, and the edge $\Gamma_{2}^{(1)}$, come from the case $a \cdot b \neq 0$. The remaining 5 families of expansions of solutions are new:

$\mathcal{C}_{\infty}^{0}$, which exists for $c=0$, is given by formula (3.7.4), and has one parameter; $\mathcal{D}_{10}$, which exists for $c=0$, is given by formula (3.7.6), and has one parameter; $\mathcal{D}_{8,9}$, which exist for $c<0$, are given by formula (3.7.10), and have one parameter;

$\mathcal{D}_{7}$, which exists for $c \neq 0$, is given by formula (3.7.9), and has two parameters.

The symmetry (3.6.1) maps expansions of solutions as $x \rightarrow 0$ to expansions of solutions as $x \rightarrow \infty$. Expansions $\mathcal{C}_{0}^{0}, \mathcal{H}_{8}-\mathcal{H}_{10}, \mathcal{H}_{7}$ are mapped to expansions $\mathcal{C}_{\infty}^{0}, \mathcal{D}_{8}-\mathcal{D}_{10}, \mathcal{D}_{7}$ respectively (and vice versa).

\section{CHAPTER 4. EXPANSIONS OF SOLUTIONS OF P6 NEAR ZERO AND INFINITY IN THE CASE $a=b=0$}

\section{Expansions}

In the case where the parameters in the sixth Painlevé equation (2.1.1) are $a=b=0$, $c$ and $d$ arbitrary, we are looking for expansions of solutions as $x \rightarrow 0$ and $x \rightarrow \infty$ of the form (2.1.2) and of all five types: power, power-logarithmic, complicated, semiexotic, and exotic.

For $a=b=0$, equation (2.1.4) takes the form

$$
\begin{aligned}
f(x, y) \stackrel{\text { def }}{=} & 2 y^{\prime \prime} x^{2}(x-1)^{2} y(y-1)(y-x) \\
& -\left(y^{\prime}\right)^{2}\left[x^{2}(x-1)^{2}(y-1)(y-x)+x^{2}(x-1)^{2} y(y-x)+x^{2}(x-1)^{2} y(y-1)\right] \\
& +2 y^{\prime}\left[x(x-1)^{2} y(y-1)(y-x)+x^{2}(x-1) y(y-1)(y-x)+x^{2}(x-1)^{2} y(y-1)\right] \\
& -\left[2 c(x-1) y^{2}(y-x)^{2}+2 d x(x-1) y^{2}(y-1)^{2}\right]=0 .
\end{aligned}
$$




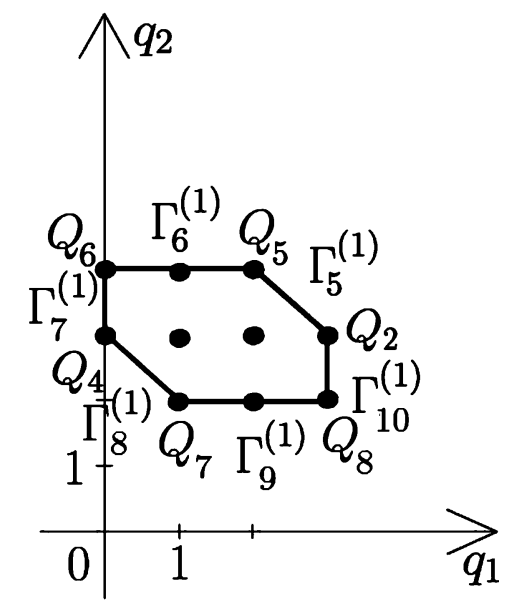

Figure 19. The support of equation (4.1.1), its polygon, vertices, and faces.

The support $\mathbf{S}\left(f_{a=b=0}\right)$ of the left-hand side of equation (4.1.1), its convex hull $\Gamma\left(f_{a=b=0}\right)$, the faces $\Gamma_{i}^{(0)} \stackrel{\text { def }}{=} Q_{i}, i=2,5,6,4,7,8$, and the edges $\Gamma_{i}^{(1)}, i=5, \ldots, 10$, are shown in Figure 19.

According to Figure 19 in this case there are no new families of expansions of solutions. All families that exist in the case $a=b=0$ are among the families in one of the cases $a \cdot b \neq 0 ; a=0, b \neq 0$, and $a \neq 0, b=0$ and are listed in Chapters 2 and 3.

Theorem 4.1.1. In the case $a=b=0$, equation (2.1.1) has 14 families of expansions of solutions as $x \rightarrow 0$. Of these, 3 families $\mathcal{A}_{0}, \mathcal{B}_{3}$ and $\mathcal{H}_{3}$ come from the case $a \cdot b \neq 0$. The remaining 11 families of expansions $\mathcal{C}_{0}^{\infty}, \mathcal{B}_{8}-\mathcal{B}_{10}, \mathcal{B}_{7}^{\tau}, \mathcal{C}_{0}^{0}, \mathcal{H}_{8}-\mathcal{H}_{10}, \mathcal{H}_{7}$ come from the cases $a=0, b \neq 0$ and $a \neq 0, b=0$.

Theorem 4.1.2. In the case $a=b=0$, equation (2.1.1) has 14 families of expansions of solutions as $x \rightarrow \infty$. Of these, 3 families $\mathcal{A}_{\infty}, \mathcal{G}_{3}$ and $\mathcal{D}_{3}$ come from the case $a \cdot b \neq 0$. The remaining 11 families of expansions $\mathcal{C}_{\infty}^{\infty}, \mathcal{G}_{8}-\mathcal{G}_{10}, \mathcal{G}_{7}^{\tau}, \mathcal{C}_{\infty}^{0}, \mathcal{D}_{8}-\mathcal{D}_{10}, \mathcal{D}_{7}$ come from the cases $a=0, b \neq 0$ and $a \neq 0, b=0$.

\section{EXAMPLES AND COMPARISON WITH PREVIOUSLY KNOWN RESUlTS}

Example 2.1. In the case

$$
a=b=c=0, \quad d=\frac{1}{2}
$$

we obtain the following basic families of expansions:

1. Three two-parameter (with respect to $c_{r}$ and $r$ ) families of expansions $\mathcal{B}_{7}^{\tau}$ with $\tau=\operatorname{sgn}(\operatorname{Im} r)$ (families of exotic expansions) and $\mathcal{A}_{0}$ (families of power expansions) given by the formula

$$
y=c_{r} x^{r}+\sum c_{s} x^{s}
$$

where the complex exponents are as follows: $r$ satisfies the conditions $\operatorname{Re} r=0, r \neq 0$ for the family $\mathcal{B}_{7}^{\tau}, \tau=\operatorname{sgn}(\operatorname{Im} r)$ and $0<\operatorname{Re} r<1$ for the family $\mathcal{A}_{0}, s \in\{r+l r+m(1-r)$; $l, m \geq 0 ; l+m>0 ; l, m \in \mathbb{Z}\}$. The complex coefficients are as follows: $c_{r}$ is an arbitrary nonzero constant, and all $c_{s}$ are uniquely determined constants. 
If $\operatorname{Re} r=0, r \neq 0$, then the sum of the series (4.2.2) for $m=0$ is the function (3.4.39); i.e., asymptotics are

$$
y=\sin ^{-2}\left[-\frac{\operatorname{Im} r}{2} \ln \left(C_{17} x\right)\right]
$$

where $r=\sqrt{-C_{2}}, C_{2}$ is an arbitrary real positive number, and $C_{17}$ is an arbitrary nonzero constant.

2. A one-parameter (in $c_{0}$ ) family of power expansions $\mathcal{B}_{10}$ is given by the formula

$$
y=c_{0}+\sum_{s=1}^{\infty} c_{s} x^{s}
$$

where the complex coefficients are as follows: $c_{0}$ is an arbitrary constant such that $c_{0} \neq 0,1, c_{1}=\frac{\left(c_{0}-1\right)}{2}$, and the remaining $c_{s}$ are uniquely determined constants.

Example 2.2. In the case (4.2.1), Picard 69] solved equation (2.1.1) explicitly. Some asymptotics of Picard's solutions can be found in [63, Lemma 2]. For $x \rightarrow 0$ these asymptotics are of the form

$$
y(x) \sim a_{0} x^{r}\left(1+O\left(x^{\varepsilon}\right)\right),
$$

where either $0<\operatorname{Re} r<1$, or $r=0$, or $r=1$, and $a_{0}$ is an arbitrary nonzero constant.

In the case $\operatorname{Re} r=0, r \neq 0$, asymptotics are [55]

$$
y(x) \sim(1+O(x)) \sin ^{-2}\left[\frac{-\operatorname{Im} r \log (x)}{2}+O(1)\right] .
$$

Comparing formula (4.2.5) with formulas (4.2.2), (4.2.4) and formula (4.2.6) with formula (4.2.3) we see that formulas (4.2.5) and (4.2.6) provide asymptotics of solutions for families $\mathcal{A}_{0}, \mathcal{B}_{10}$ (for $r=0$ ) and $\mathcal{B}_{7}^{\tau}$ respectively.

Example 2.3. For

$$
a=2, \quad b=c=0, \quad d=\frac{1}{2},
$$

we obtain the following basic families of expansions:

1. Three two-parameter families of expansions $\mathcal{B}_{0}^{\tau}$ (exotic expansions) and $\mathcal{A}_{0}$ (power expansions) given by the formula

$$
y=c_{r} x^{r}+\sum c_{s} x^{s}
$$

where the complex exponents are as follows: $r$ is an arbitrary number satisfying $\operatorname{Re} r=0$, $r \neq 0$ for the family $\mathcal{B}_{0}^{\tau}$ and $0<\operatorname{Re} r<1$ for the family $\mathcal{A}_{0}$. Also $s \in\{r+l r+m(1-r)$; $l, m \geq 0 ; l+m>0 ; l, m \in \mathbb{Z}\}$, and the complex coefficients are as follows: $c_{r}$ is an arbitrary nonzero constant, and all $c_{s}$ are uniquely determined constants.

In the case $\operatorname{Re} r=0, r \neq 0$ the sum of the series (4.2.8) for $m=0$ is the function (2.3.85), where $C_{2}$ is a real positive number, $\alpha=1, \beta=-C_{2} / 4, \gamma=\frac{\sqrt{C_{2}}}{2}$. Therefore asymptotics of solutions are

$$
y=\frac{\beta}{\beta \cos ^{2}\left[\sqrt{-\beta} \ln \left(C_{11} x\right)\right]+\sin ^{2}\left[\sqrt{-\beta} \ln \left(C_{11} x\right)\right]},
$$

where $\beta$ is an arbitrary real negative number, and $C_{11}$ is an arbitrary nonzero constant.

2. A one-parameter family of power expansions $\mathcal{B}_{6}$ given by the formula

$$
y=1+c_{2} x^{2}+\frac{c_{2}}{2} x^{3}+\sum_{s=4}^{\infty} c_{s} x^{s}
$$


where the complex coefficients are as follows: $c_{2}$ is an arbitrary nonzero constant, and all $c_{s}$ are uniquely determined constants.

3. A one-parameter family of complicated expansions $\mathcal{B}_{3}$ with asymptotics

$$
y=-\ln ^{-2} x+c_{-3} \ln ^{-3} x+\sum_{s=4}^{\infty} c_{-s} \ln ^{-s} x,
$$

where the complex coefficients are as follows: $c_{-3}$ is an arbitrary nonzero constant, and all $c_{-s}$ are uniquely determined constants.

Example 2.4. In the case (4.2.7) the explicit form of a class of solutions (called Chazy solutions) was found. The asymptotics of solutions of this class as $x \rightarrow 0$ was found in [63. Lemma 5] in the following form:

$$
y(x) \sim-\log ^{-2} x+b_{0} \log ^{-3} x+O\left(\log ^{-4} x\right),
$$

where $b_{0}$ is an arbitrary complex number.

Formula (4.2.12) is similar to (4.2.11) in the case (4.2.7). For other solutions of (4.2.7) (except the Chazy solutions) asymptotics have not been written out explicitly.

According to [63, Lemma 6] there exists a transformation that maps solutions in the case (4.2.7) to solutions in the case (4.2.1). By [63, Lemma 7], all Chazy solutions are mapped to the exceptional solution $\mathcal{I}_{4}: y=\infty$. In 63] a class of solutions (called solutions of Picard type) was described. By [63, Lemma 8] solutions of Picard type are not mapped to the exceptional solution $y=\infty$. Therefore, it is reasonable to assume that asymptotics of solutions of Picard type have the same form as solutions in families $\mathcal{A}_{0}, \mathcal{B}_{0}^{\tau}$, and $\mathcal{B}_{6}$.

Let us consider the transformation from [63, Lemma 6] for expansions of solutions in the family $\mathcal{B}_{6}$, i.e., expansions of the form (4.2.10). This transformation maps expansions (4.2.10) to expansions of the form

$$
y=\frac{1}{1-64 c_{2}}-\frac{32 c_{2}}{1-64 c_{2}} x+\cdots,
$$

where $c_{2}$ is an arbitrary complex constant from (4.2.10).

For $c_{2} \neq \frac{1}{64}$ expansion (4.2.13) belongs to family $\mathcal{B}_{10}$, i.e., has the form (4.2.4). However, in the case $c_{2}=\frac{1}{64}$ it is an exceptional solution $y=\infty$. Therefore, in the case (4.2.7), a transformation from [63, Lemma 6] maps solution (4.2.10) from the family $\mathcal{B}_{6}$ with $c_{2}=\frac{1}{64}$ to the solution $y=\infty$. By [63, Lemma 8] this solution is not a solution of Picard type. Since its asymptotics as $x \rightarrow 0$ differs from the asymptotics (4.2.11) of Chazy solutions, this solution is not a Chazy solution.

Therefore, in the case (4.2.7), there exists a solution (4.2.10) with $c_{2}=\frac{1}{64}$ that is neither a Chazy solution nor a solution of Picard type. This contradicts Theorem 4 (iii) in [63], which claims that in the case (4.2.7) Chazy solutions and Picard type solutions exhaust all possible solutions.

In fact, in the case (4.2.7) there are solutions of three types: 1) a two-parameter family of solutions of Picard type, 2) one-parameter family of Chazy solutions and 3) a unique solution (4.2.10) with $c_{2}=\frac{1}{64}$. Expansions of type 2) are limiting expansions for type 1 ), and the solution of type 3) is a limiting solution for types 1) and 2). In addition to asymptotic properties, some other properties of the solution of type 3 ) are also quite special.

\section{Chapter 5. Expansions of SOlutions of P6 NeAR the POINT $x=1$}

Using the symmetry (2.1.9) we obtain expansions of solutions for $x \rightarrow 1$ from expansions of solutions for $x \rightarrow 0$. Here we formulate the results without proofs. 


\section{EXPANSIONS NEAR 1 IN THE CASE $a \cdot c \neq 0$}

1.1. Two-parameter families of expansions. There is one two-parameter family of power expansions of solutions

$$
\mathcal{A}_{1}: \quad y=1+c_{r}(1-x)^{r}+\sum_{s} c_{s}(1-x)^{s},
$$

where the exponent $r$ is any number satisfying the condition $0<\operatorname{Re} r<1$, and $s \in$ $\{r+l r+m(1-r) ; l, m \geq 0 ; l+m>0 ; l, m \in \mathbb{Z}\}, c_{r} \neq 0, c_{r}$ is an arbitrary complex constant, and the remaining coefficients $c_{s}$ are uniquely determined constants.

For $1>\operatorname{Re} r>\frac{1}{2}$ the third approximation to a solution is $y=1+c_{r}(1-x)^{r}+c_{1}(1-x)$, where

$$
c_{1}=\frac{2(c+d)+(r-1)^{2}-1}{2(r-1)^{2}} .
$$

For $0<\operatorname{Re} r<\frac{1}{2}$ the third approximation to a solution is $y=1+c_{r}(1-x)^{r}+c_{2 r}(1-x)^{2 r}$, where

$$
c_{2 r}=-c_{r}^{2} \frac{2(a+b)+r^{2}}{2 r^{2}} .
$$

For $\operatorname{Re} r=\frac{1}{2}, \operatorname{Im} r \neq 0$ the third approximation to a solution is $y=1+c_{r}(1-x)^{r}+$ $c_{1}(1-x)+c_{2 r}(1-x)^{2 r}$, where the coefficients $c_{1}$ and $c_{2 r}$ are given by formulas (5.1.2) and (5.1.3) respectively. For $r=\frac{1}{2}$ the third approximation to a solution is $y=1+c_{\frac{1}{2}} \sqrt{1-x}+$ $c_{1}(1-x)$ where $c_{1 / 2}$ is an arbitrary constant, and $c_{1}=\left(-3+8(c+d)-c_{1 / 2}^{2}-8 c_{1 / 2}^{2}(a+b)\right) / 2$.

There exist two two-parameter families of exotic expansions $\mathcal{E}_{0}^{\tau}$ and one two-parameter family of semiexotic expansions $\mathcal{J}_{0}$ that are given by the following formula:

$$
y=1+c_{r}(1-x)^{r}+\sum_{s} c_{s}(1-x)^{s},
$$

where $x \rightarrow 1$, and the complex exponents are as follows: for the families $\mathcal{E}_{0}^{\tau}$ with $\tau=$ $\operatorname{sgn}(\operatorname{Im} r)$ the exponent $r$ is arbitrary with $\operatorname{Re} r=0$; for the family $\mathcal{J}_{0}$, the exponent $r$ satisfies $\operatorname{Re} r=1, r \neq 1$ and $s \in\{l+k(r-1) ; l \geq 1 ;|k| \leq l ; l, k \in \mathbb{Z}\}$. The complex coefficients are as follows: $c_{r}$ is an arbitrary nonzero constant, and the $c_{s}$ are uniquely determined constants.

1.2. Expansions of solutions for $a \neq-b \neq 0$. Let $\theta_{i} \stackrel{\text { def }}{=} \sqrt{2 a}+(-1)^{i} \sqrt{-2 b}$, where $i=1,2$. For each value of $i$ we have one of three possible cases, depending on the value of $\theta_{i}$.

Case $1 . \operatorname{Re} \theta_{i}=0$. In this case we have the one-parameter families of exotic expansions

$$
\mathcal{E}_{i}^{\tau}: \quad y=c_{0 i}+\sum_{s} c_{s i}(1-x)^{s}, \quad i=1,2, \quad \tau= \pm 1,
$$

where $s \in\left\{l+m \tau \theta_{i} ; l, m \geq 0 ; l+m>0 ; l, m \in \mathbb{Z}\right\}$, and the complex coefficients are as follows:

$$
c_{0 i}=(-1)^{i} \sqrt{-\frac{b}{a}},
$$

$c_{s i}$ with $s=\tau \theta_{i}$ is an arbitrary constant and the remaining $c_{s i}$ are uniquely determined constants.

If $c_{s i}=0$ with $s=\tau \theta_{i}$, then the family of power expansions (5.1.5) is denoted by $\mathcal{E}_{i}$. 
In the case $c_{s i}=0$ with $s=\tau \theta_{i}$, the second approximation to expansions of solutions (5.1.5) is $y=c_{0 i}+c_{1 i}(1-x)$, where

$$
c_{1 i}=(-1)^{i} \sqrt{\frac{-b}{a}} \frac{c+d-\left(\sqrt{a}+(-1)^{i} \sqrt{-b}\right)^{2}}{1-2\left(\sqrt{a}+(-1)^{i} \sqrt{-b}\right)^{2}} .
$$

Let $k_{i}=\theta_{i}$ for $\operatorname{Re} \theta_{i}>0$ and $k_{i}=-\theta_{i}$ for $\operatorname{Re} \theta_{i}<0$.

Case 2. $\operatorname{Re} \theta_{i} \neq 0, \theta_{i} \notin \mathbb{Z}$. Here we have a family of power expansions of solutions

$$
\mathcal{E}_{i}: \quad y=c_{0 i}+\sum_{s} c_{s i}(1-x)^{s}, \quad i=1,2,
$$

where $s \in\left\{l+m k_{i} ; l, m \in \mathbb{Z} ; l, m \geq 0 ; l+m>0\right\}$. The complex coefficients are as follows: $c_{0 i}$ is given by formula (5.1.6),,$c_{k_{i} i}$ is arbitrary, and the remaining $c_{s i}$ are uniquely determined constants. The second approximation to the expansion of solution (5.1.8) depends on the number $\operatorname{Re} k_{i}$. If $\operatorname{Re} k_{i}>1$, then the second approximation is $y=c_{0 i}+c_{1 i}(1-x)$, which is similar to the case $\operatorname{Re} \theta_{i}=0$. If $0<\operatorname{Re} k_{i}<1$, then the second approximation to a solution is $y=c_{0 i}+c_{k_{i} i}(1-x)^{k_{i}}$, where $c_{k_{i} i}$ is an arbitrary coefficient. If $\operatorname{Re} k_{i}=1$, then the second approximation to a solution is $y=c_{0 i}+c_{1 i}(1-x)+c_{k_{i} i}(1-x)^{k_{i}}$, where $c_{k_{i} i}$ is an arbitrary coefficient, and $c_{1 i}$ is given by formula (5.1.7).

Case 3. $\operatorname{Re} \theta_{i} \in \mathbb{N} \backslash\{0\}$. Here we have a family of power-logarithmic expansions of solutions:

$$
\mathcal{E}_{i}: \quad y=c_{0 i}+\sum_{s=1}^{\infty} c_{s i}(\ln (1-x))(1-x)^{s}, \quad i=1,2 .
$$

The coefficient $c_{k_{i} i}$ is given by the formula $c_{k_{i} i}=\alpha_{k_{i} i}+\beta_{k_{i} i} \ln (1-x)$, where $\alpha_{k_{i} i}$ is an arbitrary constant, $\beta_{k_{i} i}$ is a uniquely determined constant, $c_{0 i}$ is given by formula (5.1.6), and the remaining $c_{s i}$ are uniquely determined polynomials in $\ln (1-x)$. Here expansion (5.1.9) forms a one-parameter (in $\alpha_{k_{i} i}$ ) family $\mathcal{E}_{i}$. The second approximation to the expansion of solution (5.1.9) depends on the number $k_{i}$. In the case $k_{i}=1$ the second approximation to a solution is $y=c_{0 i}+c_{1 i}(1-x)$. The coefficient is given as follows:

$$
c_{1 i}=\alpha_{1 i}+(-1)^{i} \sqrt{\frac{-b}{a}} \frac{c+d-\left(\sqrt{a}+(-1)^{i} \sqrt{-b}\right)^{2}}{2} \ln (1-x),
$$

where $\alpha_{1 i}$ is an arbitrary constant.

There is a family of complicated expansions

$$
\mathcal{E}_{3}: \quad y=\varphi_{0}+\sum_{\sigma=1}^{\infty} \varphi_{\sigma}(1-x)^{\sigma}
$$

where $\varphi_{0}=1+\frac{2}{a+b} \ln ^{-2}(1-x)+c_{-3} \ln ^{-3}(1-x)+\sum_{s=4}^{\infty} c_{-s} \ln ^{-s}(1-x)$, and the complex coefficients are as follows: $c_{-3}$ is an arbitrary constant, the $c_{-s}$ are uniquely determined constants, and $\varphi_{\sigma}$ is a series in decreasing powers of logarithms.

1.3. Expansions of solutions for $a=-b \neq 0$. We have two one-parameter families of complicated expansions:

$$
\mathcal{E}_{3+j}: \quad y=\varphi_{0 j}+\sum_{\sigma=1}^{\infty} \varphi_{\sigma j}(1-x)^{\sigma}
$$

where

$$
\varphi_{0 j}=1+(-1)^{j} \frac{1}{\sqrt{2 a}} \ln ^{-1}(1-x)+c_{-2 j} \ln ^{-2}(1-x)+\sum_{s=3}^{\infty} c_{-s j} \ln ^{-s}(1-x), \quad j=1,2 .
$$


The complex coefficients are as follows: $c_{-2 j}$ is an arbitrary constant, $c_{-s j}$ are uniquely determined constants, and $\varphi_{\sigma j}$ are series in decreasing powers of logarithms.

The families of expansions $\mathcal{E}_{2}$ and $\mathcal{E}_{2}^{\tau}$ come from the case $a \neq b \neq 0$. For $\theta_{2}$ we take $2 \sqrt{2 a}$ with $\operatorname{Re} \theta_{2} \geq 0$. Depending on $\theta_{2}$ we have three possible cases: Case $1\left(\operatorname{Re} \theta_{2}=0\right.$, the one-parameter family of exotic expansions $\mathcal{E}_{2}^{\tau}$ is given by formula (5.1.5)), Case $2\left(\operatorname{Re} \theta_{2} \neq 0, \check{\theta_{2}} \notin \mathbb{Z}\right.$, the one-parameter family of power expansions $\mathcal{E}_{2}$ is given by formula (5.1.8) $)$, and Case $3\left(\theta_{2} \in \mathbb{Z} \backslash\{0\}\right.$, the one-parameter family of power-logarithmic expansions $\mathcal{E}_{2}$ is given by formula (5.1.9) $)$.

1.4. Expansions of solutions for $b=0$. By Theorem (2.1.4) in this case we have the exceptional solution

$$
\mathcal{I}_{2}: \quad y=1 .
$$

We also have one family of power expansions

$$
\mathcal{E}_{6}: \quad y=c_{\rho}(1-x)^{\rho}+\sum_{s} c_{s}(1-x)^{s}
$$

where the coefficients are as follows: $c_{\rho} \neq 0$ is an arbitrary constant, the $c_{s}$ are uniquely determined constants, $\rho=\sqrt{2 a}$, Re $\sqrt{2 a}>0$, and $s \in\{\rho+l \rho+m ; l, m \geq 0 ; l+m>0$; $l, m \in \mathbb{Z}\}$.

If $\operatorname{Re} \rho>1$, then the second approximation to a solution is $y=c_{\rho}(1-x)^{\rho}+$ $c_{\rho+1}(1-x)^{\rho+1}$ with the coefficient given as follows:

$$
c_{\rho+1}=c_{\rho} \frac{a-c-d}{\rho+1} .
$$

If $0<\operatorname{Re} \rho<1$, then the second approximation to a solution is $y=c_{\rho}(1-x)^{\rho}+c_{2 \rho}(1-x)^{2 \rho}$ with the coefficient given as follows:

$$
c_{2 \rho}=-c_{\rho}^{2} .
$$

If $\operatorname{Re} \rho=1, \operatorname{Im} \rho \neq 0$, then the second approximation to a solution is $y=c_{\rho}(1-x)^{\rho}+$ $c_{2}(1-x)^{2}+c_{2 \rho}(1-x)^{2 \rho}$ with coefficients $c_{2 \rho}$ and $c_{2}$ given by formulas (5.1.14) and (5.1.15) respectively. If $\rho=1$, then the second approximation to a solution is $y=$ $c_{1}(1-x)+c_{2}(1-x)^{2}$. The complex coefficients are given as follows: $c_{1}$ is an arbitrary constant and

$$
c_{2}=c_{1} \frac{a-c-d-2 c_{1}}{2}
$$

is the sum of (5.1.14) and (5.1.15).

Here we have two one-parameter families of exotic expansions:

$$
\mathcal{E}_{6}^{\tau}: \quad y=c_{\rho}(1-x)^{\rho}+\sum_{s} c_{s}(1-x)^{s},
$$

where $\rho=\sqrt{2 a}, \operatorname{Re} \rho=0, s$ runs over the set $\{\rho+l \rho+m ; l, m \geq 0 ; l+m>0 ; l, m \in \mathbb{Z}\}$, $\tau=\operatorname{sgn}(\operatorname{Im} \rho)$, and the complex coefficients are as follows: $c_{\rho}$ is an arbitrary nonzero constant, and all $c_{s}$ are uniquely determined constants.

Also, we have a one-parameter family of complicated expansions $\mathcal{E}_{3}$ given by formula (5.1.11).

1.5. Expansions of solutions in the case $-c \neq d-\frac{1}{2} \neq 0$. Set $\theta_{i} \stackrel{\text { def }}{=} \sqrt{1-2 d}+$ $(-1)^{i} \sqrt{2 c}$, where $i=1,2$. For each $i$, depending on the value of $\theta_{i}$, three cases are possible.

Case 1. $\operatorname{Re} \theta_{i}=0$. We have the one-parameter families of semiexotic expansions of solutions

$$
\mathcal{J}_{i}^{\tau}: \quad y=1+c_{1 i}(1-x)^{\tau \theta_{i}}+\sum_{s}^{\infty} c_{s i}(1-x)^{s}, \quad i=1,2, \quad \tau= \pm 1,
$$


where $s \in\left\{1+l+m \tau \theta_{i} ; l, m \geq 0 ; l+m>0 ; l, m \in \mathbb{Z}\right\}$ and the complex coefficients are given as follows:

$$
c_{1 i}=\frac{-2 c-(-1)^{i} \sqrt{-4 c d+2 c}}{2 c+2 \check{d}-1},
$$

the $c_{s i}$ with $s=1+\tau \theta_{i}$ are arbitrary constants, and the $c_{s i}$ are uniquely determined constants.

For $c_{s i}=0$ with $s=1+\tau \theta_{i}$ the family of power expansions (5.1.17) is denoted by $\mathcal{J}_{i}$.

For $c_{s i}=0$ with $s=1+\tau \theta_{i}$ the third approximation (5.1.17) is $y=1+c_{1 i}(1-x)+$ $c_{2 i}(1-x)^{2}$, where

$$
c_{2 i}=(-1)^{i} \sqrt{\frac{1-2 d}{2 c}} \frac{2(a+b)+1-\left(\sqrt{2 c}+(-1)^{i} \sqrt{1-2 d}\right)^{2}}{2-2\left(\sqrt{2 c}+(-1)^{i} \sqrt{1-2 d}\right)^{2}} .
$$

Let $k_{i}=1+\theta_{i}$ if $\operatorname{Re} \theta_{i}>0$ and $k_{i}=1-\theta_{i}$ if $\operatorname{Re} \theta_{i}<0$.

Case $2 . \operatorname{Re} \theta_{i} \neq 0, \theta_{i} \notin \mathbb{Z}$. We have the following family of power expansions of solutions:

$$
\mathcal{J}_{i}: \quad y=1+c_{1 i}(1-x)+\sum_{s} c_{s i}(1-x)^{s}
$$

where $s \in\left\{1+l+m k_{i} ; l, m \geq 0 ; l+m>0 ; l, m \in \mathbb{Z}\right\}$ and the complex coefficients are as follows: $c_{1 i}$ is given by formula (5.1.18),,$c_{k_{i} i}$ is arbitrary, and the remaining $c_{s i}$ are uniquely determined constants.

The second approximation to the expansion of solution (5.1.20) depends on $\operatorname{Re} k_{i}$. If $\operatorname{Re} k_{i}>2$, then the second approximation to a solution is $y=1+c_{1 i}(1-x)+c_{2 i}(1-x)^{2}$, which is similar to the case $\operatorname{Re} \theta_{i}=0$. If $1<\operatorname{Re} k_{i}<2$, then the second approximation to a solution is $y=1+c_{1 i}(1-x)+c_{k_{i} i}(1-x)^{k_{i}}$, where $c_{k_{i}}$ is an arbitrary coefficient. If $\operatorname{Re} k_{i}=2$, then the second approximation to a solution is $y=1+c_{1 i}(1-x)+c_{2 i}(1-x)^{2}+$ $c_{k_{i} i}(1-x)^{k_{i}}$, where $c_{k_{i} i}$ is arbitrary and $c_{2 i}$ is given by formula (5.1.19).

Case 3 . Re $\theta_{i} \in \mathbb{N} \backslash\{0\}$. We have the following family of power-logarithmic expansions of solutions:

$$
\mathcal{J}_{i}: \quad y=1+c_{1 i}(1-x)+\sum_{s=2}^{\infty} c_{s i}(\ln (1-x))(1-x)^{s},
$$

where the complex coefficients are as follows: $c_{1 i}$ is given by formula (5.1.18), $c_{k_{i} i}=$ $\alpha_{k_{i} i}+\beta_{k_{i} i} \ln (1-x)$, where $\alpha_{k_{i} i}$ is an arbitrary constant, and the remaining $c_{s i}$ and $\beta_{k_{i} i}$ are uniquely determined constants.

The second approximation to the expansion of solutions (5.1.21) depends on $k_{i}$. If $k_{i}=2$, then the second approximation of a solution is $y=1+c_{1 i}(1-x)+c_{2 i}(1-x)^{2}$, where

$$
c_{2 i}=\alpha_{2 i}+(-1)^{i} \sqrt{\frac{1-2 d}{2 c}} \frac{2(a+b)+1-\left(\sqrt{2 c}+(-1)^{i} \sqrt{1-2 d}\right)^{2}}{4} \ln (1-x),
$$

and $\alpha_{2 i}$ is an arbitrary constant.

Here we have a family of complicated expansions

$$
\mathcal{J}_{3}: \quad y=1+\varphi_{1}(1-x)+\sum_{\sigma=2}^{\infty} \varphi_{\sigma}(1-x)^{\sigma}
$$

where

$$
\varphi_{1}=-\frac{1-2 c-2 d}{4} \ln ^{2}(1-x)+c_{1} \ln (1-x)+\sum_{s=0}^{\infty} c_{-s} \ln ^{-s}(1-x)
$$


and the complex coefficients are as follows: $c_{1}$ is an arbitrary constant, and the remaining $c_{-s}$ are uniquely determined constants. Also, $\varphi_{\sigma}$ are series in decreasing powers of logarithms.

1.6. Expansions of solutions for $-c=d-\frac{1}{2} \neq 0$. We have two families of complicated expansions:

$$
\mathcal{J}_{3+j}: \quad y=1+\varphi_{1 j}(1-x)+\sum_{\sigma=2}^{\infty} \varphi_{\sigma j}(1-x)^{\sigma}
$$

where

$$
\varphi_{1 j}=(-1)^{j} \sqrt{2 c} \ln (1-x)+c_{0 j}+\sum_{s=1}^{\infty} c_{-s j} \ln ^{-s}(1-x), \quad j=1,2,
$$

and the complex coefficients are given as follows: $c_{0 j}$ is an arbitrary constant, and the remaining $c_{-s j}$ are uniquely determined constants. Also, $\varphi_{\sigma j}$ are series in decreasing powers of logarithms.

The families $\mathcal{J}_{2}, \mathcal{J}_{2}^{\tau}$ come from the case $c \neq \frac{1}{2}-d \neq 0$. For $\theta_{2}$ we take $2 \sqrt{2 c}$ with $\operatorname{Re} \theta_{2} \geq 0$. Depending on the value of $\theta_{2}$ we have three cases: Case $1\left(\operatorname{Re} \theta_{2}=0\right.$, one-parameter families of exotic expansions $\mathcal{J}_{2}^{\tau}$ are given by formula (5.1.17)), Case 2 $\left(\operatorname{Re} \theta_{2} \neq 0, \theta_{2} \notin \mathbb{Z}\right.$, one-parameter family of exotic expansions $\mathcal{J}_{2}$ is given by formula $(5.1 .20)$ ), and Case $3\left(\theta_{2} \notin \mathbb{Z}\right.$, one-parameter family of power-logarithmic expansions $\mathcal{J}_{2}$ is given by formula (5.1.21) $)$.

1.7. Expansions of solutions for $d=\frac{1}{2}$. Here we have one family of power expansions

$$
\mathcal{J}_{6}: \quad y=x+c_{\rho}(1-x)^{\rho}+\sum_{s} c_{s}(1-x)^{s},
$$

where $c_{\rho} \neq 0, c_{\rho}$ is an arbitrary constant, $\rho=1+\sqrt{2 c}$, Re $\sqrt{2 c}>0, s$ runs over the set $\{\rho+l(\rho-1)+m ; l, m \geq 0 ; l+m>0 ; l, m \in \mathbb{Z}\}$, and the remaining complex coefficients $c_{s}$ are uniquely determined constants.

In the case $\operatorname{Re} \rho>2$, the third approximation to expansion (5.1.27) is $y=x+$ $c_{\rho}(1-x)^{\rho}+c_{\rho+1}(1-x)^{\rho+1}$, with the coefficient given by the formula

$$
c_{\rho+1}=-c_{\rho} \frac{-2 c+2 a+2 b+1}{2 \rho} .
$$

In the case $1<\operatorname{Re} \rho<2$ the third approximation to expansion (5.1.27) takes the form $y=x+c_{\rho}(1-x)^{\rho}+c_{2 \rho-1}(1-x)^{2 \rho-1}$. The coefficient is given as follows:

$$
c_{2 \rho-1}=c_{\rho}^{2} \text {. }
$$

In the case $\operatorname{Re} \rho=2, \operatorname{Im} \rho \neq 0$ the third approximation to expansion (5.1.27) is of the form $y=x+c_{\rho}(1-x)^{\rho}+c_{\rho+1}(1-x)^{\rho+1}+c_{2 \rho-1}(1-x)^{2 \rho-1}$, where the coefficients $c_{\rho+1}$ and $c_{2 \rho-1}$ are given by formulas (5.1.28) and (5.1.29) respectively. In the case $\rho=2$ the third approximation to expansion (5.1.27) is of the form $y=x+c_{2}(1-x)^{2}+$ $c_{3}(1-x)^{3}$. The coefficients are as follows: $c_{2}$ is an arbitrary nonzero constant, and $c_{3}=-c_{2} \frac{\left(-2 c+2 a+2 b+1-4 c_{2}\right)}{4}$ is the sum of (5.1.28) and (5.1.29).

We have two one-parameter families of semiexotic expansions

$$
\mathcal{J}_{6}^{\tau}: \quad y=x+c_{\rho}(1-x)^{\rho}+\sum_{s} c_{s}(1-x)^{s},
$$

where $\rho=1 \pm \sqrt{2 c}, \operatorname{Re} \rho=1, s$ sums over the set $\{\rho+l(\rho-1)+m ; l, m \geq 0 ; l+m>0$; $l, m \in \mathbb{Z}\}, \tau=\operatorname{sgn}(\operatorname{Im} \rho)$, and the complex coefficients are as follows: $c_{\rho}$ is an arbitrary nonzero constant, and all $c_{s}$ are uniquely determined constants. 
According to Theorem 2.1.4 there is also an exceptional solution

$$
\mathcal{I}_{3}: \quad y=x
$$

Also, there is a one-parameter family of complicated expansions $\mathcal{J}_{3}$, given by formulas (5.1.22), (5.1.24).

\section{EXPANSIONS NEAR THE POINT 1 FOR $a \cdot c=0$}

For $x \rightarrow 1$, in the case $a=0, c \neq 0$ the following families come from the case $a \cdot c \neq 0$ : $\mathcal{A}_{1}, \mathcal{J}_{i}, i=1, \ldots, 6, \mathcal{J}_{0}, \mathcal{J}_{1}^{\tau}, \mathcal{J}_{2}^{\tau}$ and $\mathcal{J}_{6}^{\tau}, \tau= \pm 1$ and $\mathcal{E}_{3}$. In the case $a \neq 0, c=0$ the families coming from the case $a \cdot c \neq 0$ are $\mathcal{A}_{1}, \mathcal{E}_{i}, i=1, \ldots, 6, \mathcal{E}_{0}^{\tau}, \mathcal{E}_{1}^{\tau}, \mathcal{E}_{2}^{\tau}, \mathcal{E}_{6}^{\tau}$ and $\mathcal{J}_{3}$.

2.1. Families of expansion near the point 1 that are symmetric to families $\mathcal{C}_{0}^{\infty}$, $\mathcal{B}_{7}^{\tau}, \mathcal{B}_{8,9}, \mathcal{C}_{0}^{0}, \mathcal{H}_{7}, \mathcal{H}_{8,9}$. For $a=0$ we have the one-parameter family of expansions of solutions

$$
\mathcal{C}_{1}^{\infty}: \quad y=1+c_{r}(1-x)^{r}+\sum_{s} c_{s}(1-x)^{s}
$$

where $r=\sqrt{-2 b}$ satisfies the condition $\operatorname{Re} \sqrt{-2 b}<0, s \in\{r-l r+m ; l, m \geq 0$; $l+m>0 ; l, m \in \mathbb{Z}\}$, with the following complex coefficients: $c_{r}$ is an arbitrary nonzero constant, and the other $c_{s}$ are uniquely determined constants.

For $-1 \leq \operatorname{Re} \sqrt{-2 b}$ the second approximation to the solution is $y=1+c_{r}(1-x)^{r}+$ $c_{r+1}(1-x)^{r+1}$, where

$$
c_{r+1}=\frac{c_{r}(c+d-b-r)}{r-1} .
$$

If $0>\operatorname{Re} \sqrt{-2 b}>-1$, then the third approximation to the solution is $y=1+$ $c_{r}(1-x)^{r}+c_{0}$, where $c_{0}=0$.

For $a=0$ we have two two-parameter families of exotic expansions

$$
\mathcal{E}_{7}^{\tau}: \quad y=1+c_{r}(1-x)^{r}+\sum_{s} c_{s}(1-x)^{s}
$$

where $r$ is an arbitrary purely imaginary constant, $r \neq \sqrt{-2 b}, \tau=\operatorname{sgn} \operatorname{Im} r, s$ runs over the set $\{r+l r+m(1-r) ; l, m \geq 0 ; l+m>0 ; l, m \in \mathbb{Z}\}$, and the complex coefficients are as follows: $c_{r}$ is an arbitrary nonzero constant, and the $c_{s}$ are uniquely determined constants.

For $a=0, b>0$ we have two one-parameter families of expansions of solutions:

$$
\mathcal{E}_{8,9}: \quad y=1+c_{r}(1-x)^{r}+\sum_{s} c_{s}(1-x)^{s}
$$

where $r=\sqrt{-2 b}, \operatorname{Re} r=0, \operatorname{Im} r>0$ for the family $\mathcal{E}_{8}$ and $\operatorname{Im} r<0$ for the family $\mathcal{E}_{9}$. The exponent $s$ runs over the set $\{r+l(1-r)+m ; l, m \geq 0 ; l+m>0 ; l, m \in \mathbb{Z}\}$. The complex coefficients are as follows: $c_{r}$ is an arbitrary nonzero constant, and the $c_{s}$ are uniquely determined constants.

For $c=0$ we have the following expansions of solutions:

$$
\mathcal{C}_{1}^{1}: \quad y=1+c_{r}(1-x)^{r}+\sum_{s} c_{s}(1-x)^{s}
$$

where $r=1+\sqrt{1-2 d}$ satisfies the condition $\operatorname{Re} \sqrt{1-2 d}>0, s \in\{r+l(r-1)+m$; $l, m>0 ; l+m \geq 0 ; l, m \in \mathbb{Z}\}$, and the complex coefficients are as follows: $c_{r}$ is an arbitrary nonzero constant, and the $c_{s}$ are uniquely determined constants.

In the case $\operatorname{Re} \sqrt{1-2 d} \geq 1$ the third approximation is $y=1+c_{r}(1-x)^{r}+$ $c_{2 r-1}(1-x)^{r+1}, c_{r+1}=c_{r} \frac{(a+b-d+r)}{r}$; in the case $0<\operatorname{Re} \sqrt{1-2 d}<1$ the third approximation is $y=1+c_{r}(1-x)^{r}+c_{2 r-1}(1-x)^{2 r-1}, c_{2 r-1}=0$. 
For $c=0$ we have one two-parameter family of semiexotic expansions of solutions

$$
\mathcal{J}_{7}: \quad y=1+c_{r}(1-x)^{r}+c_{1}(1-x)+c_{2-r}(1-x)^{2-r}+\sum_{s} c_{s}(1-x)^{s},
$$

where $r-1$ is an arbitrary purely imaginary constant, $r \neq 1, r \neq \sqrt{2 a}, s$ runs over the set $\{l+k(r-1) ; l \geq 2 ;|k| \leq l ; l, k \in \mathbb{Z}\}$, and the complex coefficients are as follows: $c_{r}$ is an arbitrary nonzero constant, and $c_{1}, c_{2-r}$, and $c_{s}$ are uniquely determined constants.

For $c=0, d>\frac{1}{2}$ we have two one-parameter families of expansions

$$
\mathcal{J}_{8,9}: \quad y=1+c_{r}(1-x)^{r}+\sum_{s} c_{s}(1-x)^{s}
$$

where $r=1+\sqrt{1-2 d}$ satisfies the condition $\operatorname{Re} r=1, \operatorname{Im} r<0$ for the family $\mathcal{J}_{8}$, $\operatorname{Im} r>0$ for the family $\mathcal{J}_{9}$, the exponent $s$ runs over the set $\{r+l(1-r)+m ; l, m \geq$ $0 ; l+m>0 ; l, m \in \mathbb{Z}\}$, and the complex coefficients are as follows: $c_{r}$ is an arbitrary nonzero constant, and the $c_{s}$ are uniquely determined constants.

2.2. Expansions of solutions in the case $a=b=0, c \neq 0$. We have a one-parameter family of expansions

$$
\mathcal{E}_{10}: \quad y=c_{0}+\sum_{s=1}^{\infty} c_{s}(1-x)^{s}
$$

where $c_{0} \neq 0,1$ is an arbitrary complex number, and the remaining complex coefficients $c_{s}$ are uniquely determined constants.

The third approximation to the expansion of a solution is $y=1+c_{0}+c_{1}(1-x)$, where

$$
c_{1}=-c_{0}(c+d) \text {. }
$$

2.3. Expansions of solutions in the case $a \neq 0, c=0, d=\frac{1}{2}$. We have a oneparameter family of expansions

$$
\mathcal{J}_{10}: \quad y=1+c_{1}(1-x)+\sum_{s=2}^{\infty} c_{s}(1-x)^{s},
$$

where $c_{1}$ is an arbitrary complex number, $c_{1} \neq 0,1, c_{2}=-c_{1}\left(c_{1}+1\right)(1+2 a+2 b)$, and the remaining $c_{s}$ are uniquely determined complex constants.

2.4. Expansions of solutions in the case $a=c=0$. In this case equation (2.1.1) has 14 families of expansions of solutions as $x \rightarrow 1$. Three of these families, $\mathcal{A}_{1}, \mathcal{E}_{3}$ and $\mathcal{J}_{3}$, come from the case $a \cdot c \neq 0$. The remaining 11 families $\mathcal{C}_{1}^{\infty}, \mathcal{E}_{8}-\mathcal{E}_{10}, \mathcal{E}_{7}^{\tau}, \mathcal{C}_{1}^{1}, \mathcal{J}_{8}-\mathcal{J}_{10}, \mathcal{J}_{7}$ are coming from the cases $a=0, c \neq 0$ and $a \neq 0, c=0$.

\section{Summary of the Results}

Theorem 5.3.1. As $x \rightarrow 1$ equation (2.1.1) has 21 families of expansions of solutions of types $1-3$ :

$\mathcal{A}_{1}=\mathcal{A}_{0}^{\circ}$, which is defined by formula (5.1.1), and has two parameters;

$\mathcal{E}_{1}=\mathcal{B}_{1}^{\circ}$, which exists for $a \neq-b \neq 0$, is defined by formulas (5.1.5), (5.1.8), (5.1.9), and has 1 or 0 parameters;

$\mathcal{E}_{2}=\mathcal{B}_{2}^{\circ}$, which exists for $b \neq 0$, is defined by formulas (5.1.5), (5.1.8), (5.1.9) and has 1 or 0 parameters;

$\mathcal{E}_{3}=\mathcal{B}_{3}^{\circ}$, which exists for $a \neq-b$, is defined by formula (5.1.11), and has one parameter; 
$\mathcal{E}_{4}=\mathcal{B}_{4}^{\circ}$ and $\mathcal{E}_{5}=\mathcal{B}_{5}^{\circ}$, which exist for $a=-b \neq 0$, are defined by formula (5.1.12), and have one parameter;

$\mathcal{E}_{6}=\mathcal{B}_{6}^{\circ}$, which exists for $b=0$, is defined by formula (5.1.13), and has one parameter;

$\mathcal{C}_{1}^{\infty}=\mathcal{C}_{0}^{\infty 0}$, which exists for $a=0$, is defined by formula (5.2.1), and has one parameter;

$\mathcal{E}_{8}=\mathcal{B}_{8}^{\circ}$ and $\mathcal{E}_{9}=\mathcal{B}_{9}^{\circ}$, which exist for $b>0, a=0$, are defined by formula (5.2.3), and have one parameter;

$\mathcal{E}_{10}=\mathcal{B}_{10}^{\circ}$, which exists for $a=b=0$, is defined by formula (5.2.7), and has one parameter;

$\mathcal{J}_{1}=\mathcal{H}_{1}^{\circ}$, which exists for $a \neq-d+\frac{1}{2} \neq 0$, is defined by formulas (5.1.17), (5.1.20), (5.1.21), and has one parameter;

$\mathcal{J}_{2}=\mathcal{H}_{2}^{\circ}$, which exists for $d \neq \frac{1}{2}$, is defined by formulas (5.1.17), (5.1.20), (5.1.21), and has 1 or 0 parameters;

$\mathcal{J}_{3}=\mathcal{H}_{3}^{\circ}$, which exists for $a \neq-d+\frac{1}{2} \neq 0$, is defined by formula (5.1.23), and has one parameter;

$\mathcal{J}_{4}=\mathcal{H}_{4}^{\circ}$ and $\mathcal{J}_{5}=\mathcal{H}_{5}^{\circ}$, which exist for $a=-d+\frac{1}{2} \neq 0$, are defined by formula (5.1.25), and have one parameter;

$\mathcal{J}_{6}=\mathcal{H}_{6}^{\circ}$, which exists for $d=\frac{1}{2}$, is defined by formula (5.1.27), and has one parameter;

$\mathcal{C}_{1}^{1}=\mathcal{C}_{0}^{0 \circ}$, which exists for $c=0$, is defined by formula (5.2.4), and has one parameter;

$\mathcal{J}_{8}=\mathcal{H}_{8}^{\circ}$ and $\mathcal{J}_{9}=\mathcal{H}_{9}^{\circ}$, which exist for $c=0, d>\frac{1}{2}$, are defined by formula (5.2.6), and have one parameter;

$\mathcal{J}_{10}=\mathcal{H}_{10}^{\circ}$, which exists for $c=0, d=\frac{1}{2}$, is defined by formula (5.2.9), and has one parameter;

two families of semiexotic expansions:

$\mathcal{J}_{0}=\mathcal{H}_{0}^{\circ}$, which is defined by formula (5.1.4) and has two parameters;

$\mathcal{J}_{7}=\mathcal{H}_{7}^{\circ}$, which exists for $c=0$, is defined by formula (5.2.5), and has two parameters;

five pairs of families of exotic expansions with $\tau= \pm 1$ :

$\mathcal{E}_{0}^{\tau}=\mathcal{B}_{0}^{\tau \circ}$, which are defined by formula (5.1.4) and have two parameters;

$\mathcal{E}_{1}^{\tau}=\mathcal{B}_{1}^{\tau \circ}$, which exist for $a \neq-b \neq 0, \operatorname{Re}(\sqrt{2 a}-\sqrt{-2 b})=0$, are defined by formula (5.1.5), and have one parameter;

$\mathcal{E}_{2}^{\tau}=\mathcal{B}_{2}^{\tau \circ}$, which exist for $a \neq 0,-b \neq 0, \operatorname{Re}(\sqrt{2 a}+\sqrt{-2 b})=0$, are defined by formula (5.1.5), and have one parameter;

$\mathcal{E}_{6}^{\tau}=\mathcal{B}_{6}^{\tau \circ}$, which exist for $\operatorname{Re} \sqrt{2 a}=0, b=0$, are defined by formula (5.1.16), and have one parameter;

$\mathcal{E}_{7}^{\tau}=\mathcal{B}_{7}^{\tau \circ}$, which exists for $a=0$, is defined by formula (5.2.2), and has two parameters;

and three pairs of families of semiexotic expansions with $\tau= \pm 1$ :

$\mathcal{J}_{1}^{\tau}=\mathcal{H}_{1}^{\tau \circ}$, which exist for $-c \neq d-\frac{1}{2} \neq 0, \operatorname{Re}(\sqrt{1-2 d}-\sqrt{2 c})=0$, are defined by formula (5.1.17), and have one parameter;

$\mathcal{J}_{2}^{\tau}=\mathcal{H}_{2}^{\tau \circ}$, which exist for $c \neq 0, d \neq \frac{1}{2}, \operatorname{Re}(\sqrt{1-2 d}+\sqrt{2 c})=0$, are defined by formula (5.1.17), and have one parameter; 
$\mathcal{J}_{6}^{\tau}=\mathcal{H}_{6}^{\tau \circ}$, which exist for $\operatorname{Re} \sqrt{2 c}=0, d=\frac{1}{2}$, are defined by formula (5.1.30), and have one parameter.

$\mathcal{A}_{0}^{\circ}, \mathcal{B}_{i}^{\circ}$ and $\mathcal{H}_{i}^{\circ}$ denote the families obtained from families $\mathcal{A}_{0}, \mathcal{B}_{i}$ and $\mathcal{H}_{i}$ using symmetry (2.1.9). Families $\mathcal{A}_{1}, \mathcal{E}_{1}, \mathcal{E}_{2}, \mathcal{E}_{6}, \mathcal{E}_{8}-\mathcal{E}_{10}, \mathcal{J}_{1}, \mathcal{J}_{2}, \mathcal{J}_{6}, \mathcal{J}_{8}-\mathcal{J}_{10}$ are of types 1 and 2 ; families $\mathcal{E}_{3}, \mathcal{E}_{4}, \mathcal{E}_{5}, \mathcal{J}_{3}, \mathcal{J}_{4}, \mathcal{J}_{5}$ are complicated.

\section{REFERENCES}

[1] Ablowitz, Mark J., Segur, Harvey, Solitons and the inverse scattering transform. SIAM, Philadelphia, PA, 1981. MR642018(84a:35251)

[2] Bruno [Bryuno], A. D., Local methods in nonlinear differential equations, Fizmatlit, Moscow, 1979; English transl., Springer-Verlag, Berlin, 1989. MR.993771 (90c:58150)

[3] _ Power geometry in algebraic and differential equations. Nauka, Moscow, 1979; English transl., North-Holland, Amsterdam, 2000. MR.1773512 (2002c:37071)

[4] , Power expansions of solutions of an algebraic or a differential equation. Dokl. Akad. Nauk 380 (2001), no. 2, 155-159. (Russian) MR.1873273 (2002j:34006)

[5] (2003), no. 3, 295-300. (Russian) MR2088468 (2005g:34120)

[6] - Power-logarithmic expansions of solutions of an ordinary differential equation. (Russian) Dokl. Akad. Nauk 392 (2003), no. 4, 439-444. MR2081499

[7] _ Nonpower asymptotics of solutions of an ordinary differential equation. Dokl. Akad. Nauk 392 (2003), no. 5, 586-591. (Russian) MR 2082848 (2005e:34155)

[8] _ Asymptotic behavior and expansions of solutions of an ordinary differential equation. Uspekhi Mat. Nauk 59 (2004), no. 3(357), 31-80; English transl., Russian Math. Surveys 59 (2004), no. 3, 429-480. MR2116535 (2005m:34008)

[9] Compound expansions of solutions of an ordinary differential equation. Dokl. Akad. Nauk 406 (2006), no. 6, 730-733. (Russian) MR2347360 (2008g:34005)

[10] Exotic expansions of solutions of an ordinary differential equation. Dokl. Akad. Nauk 416 (2007), no. 5, 583-587. (Russian) MR2458919 (2009f:34135)

[11] _ On exotic expansions of solutions of an ordinary differential equation. Preprint, Keldysh Inst. Applied Math., 2010, no. 27, 27 pp. (Russian)

[12] Bruno [Bryuno], A. D., Goryuchkina, I. V., Expansions of solutions of the sixth Painlevé equation. Dokl. Akad. Nauk 395 (2004), no. 6, 733-737. (Russian) MR2114829 (2005i:34068)

[13] Expansions of solutions of the sixth Painlevé equation near a nonsingular point. Preprint, Keldysh Inst. Applied Math., 2005, no. 4, 19 pp. (Russian)

[14] Expansions of solutions of the sixth Painlevé equation in the cases $a=0$ and $b=0$. Dokl. Akad. Nauk 410 (2006), no. 3, 331-334. (Russian) MR2450920(2009f:34227)

[15] All the asymptotic expansions of solutions of the sixth Painlevé equation. Dokl. Akad. Nauk 417 (2007), no. 3, 298-302. (Russian) MR2455353 (2009f:34136)

[16] _ A survey of all asymptotic expansions of solutions of the equation P6. Preprint, Keldysh Inst. Applied Math., 2007, no. 60, 16 pp. (Russian)

[17] Methods of analysis of asymptotic expansions of solutions of the equation P6. Preprint, Keldysh Inst. Applied Math., 2007, no. 61, 30 pp. (Russian)

[18] - All basic asymptotic expansions of solutions of the equation P6 in the case $a \cdot b \neq 0$. Preprint, Keldysh Inst. Applied Math., 2007, no. 62, 30 pp. (Russian)

[19] All asymptotic expansions of solutions of the equation P6 in the case $a \cdot b=0$. Preprint, Keldysh Inst. Applied Math., 2007, no. 70, 30 pp. (Russian)

[20] _ All asymptotic expansions of solutions of the equation P6 obtained from basic ones. Preprint, Keldysh Inst. Applied Math., 2007, no. 77, 28 pp. (Russian)

[21] _ Boutroux asymptotics of solutions of Painlevé equations and power geometry. Dokl. Akad. Nauk 422 (2008), no. 2, 157-160. (Russian) MR2477348 (2009i:34200)

[22] _ Asymptotic behavior of the solutions of the third Painlevé equation. Dokl. Akad. Nauk 422 (2008), no. 6, 729-732. (Russian) MR 2477241(2009i:34123)

[23] _ Asymptotic behavior of the solutions of the fourth Painlevé equation. Dokl. Akad. Nauk 423 (2008), no. 4, 443-448. (Russian) MR2498533 (2009j:34211)

[24] - All the asymptotic expansions of solutions of the sixth Painlevé equation near its nonsingular point. Dokl. Akad. Nauk 425 (2009), no. 6, 727-732. (Russian) MR2573669 (2010h:34184) 
[25] B Basic expansions of solutions of the sixth Painlevé equation in the generic case. Differ. Uravn. 45 (2009), no. 1, 19-33. English transl., Differ. Equ. 45 (2009), no. 1, 18-32. MR2597090 (2010m:34089)

[26] Bruno [Bryuno], A. D., Chukhareva, I. V., Power expansions of solutions of the sixth Painlevé equation. Preprint, Keldysh Inst. Applied Math., 2003, no. 49. (Russian)

[27] Bruno [Bryuno], A. D., Shadrina, T. V., An axisymmetric boundary layer on a needle. Tr. Mosk. Mat. Obs. 68 (2007), 224-287; English transl., Trans. Moscow Math. Soc. 2007, 201-259. MR 2429271 (2009f:76050)

[28] Goryuchkina, I. V., Expansions of solutions of the sixth Painlevé equation in power series in real power of $x$. Differentsialnye Uravneniya 41 (2004), no. 6, 854. (Russian)

[29] _ On power and logarithmic expansions of solutions of the sixth Painlevé equation near singular points. Abstracts of talks, XXVI Conf. of Young Scientists, Math. Department, Moscow State Univ., Moscow, 2004, pp. 39-40. (Russian)

[30] _ On power and logarithmic expansions of solutions of the sixth Painlevé equation near singular points. Proc. XXVI Conf. of Young Scientists, Math. Department Moscow State Univ. Iz-vo MGU, Moscow, 2004, pp. 63-68. (Russian)

[31] _ Power and logarithmic expansions of solutions of the sixth Painlevé equation, in: Modern Methods of the Theory of Boundary Problems, VGU, Voronezh, 2004, pp. 63-64. (Russian)

[32] _ Expansions of solutions of the sixth Painlevé equation near singularities, in: Intern. Conf. on Differential Equations and Dynamical Systems, Suzdal, July 10-15, 2006, Sobor, Vladimir, 2006, pp. 75-77. (Russian)

[33] _ Exotic expansions of solutions of the sixth Painlevé equation. Preprint, Keldysh Inst. Applied Math., 2007, no. 3, 29 pp. (Russian)

[34] Goursat, Édouard, Cours d'analyse mathématique, Tomes I-III. Éditions Jacques Gabay, Sceaux, 1992.

[35] Fokas, A. S., Its, A. R., Kapaev, A. A., Novokshenov, V. Yu., Painlevé transcendents. The Riemann-Hilbert approach. Amer. Math. Soc., Providence, RI, 2006. MR.2264522 (2010e:33030)

[36] Kovalevskaya, S., Sur le problème de la rotation d'un corps solide autour d'un point fixe. Acta Math. 12 (1889) 177-232. MR.1554772

[37] Kudryashov N. A., Analytic theory of nonlinear differential equations. Inst. Computer Research, Izhevsk, 2004

[38] Kuznetsov, A. N., On the existence of solutions, entering at a singular point, of an autonomous system that has a formal solution. Funktsional. Anal. i Prilozhen. 23 (1989), no. 4, 63-74; English transl., Funct. Anal. Appl. 23 (1989), no. 4, 308-317 (1990). MR.1035375 (91b:58174)

[39] Rozov, M. Kh., Painlevé equation, Encyclopedia Math., Sovetskaya Entsiklopediya, Moscow, 1984, vol. 4, pp. 233-234. (Russian)

[40] Tikhomirov, V. M., Fréchet derivative. Encyclopedia Math., Sovetskaya Entsiklopediya, Moscow, 1984, vol. 5, p. 666. (Russian)

[41] Chukhareva, I. V., Singularities of solutions of the VI Painlevé equation. Intern. Youth Conf "Gagarinskie Chteniya XXX", Abstracts of talks, Moscow, 2004, pp. 72-73. (Russian)

[42] Ablowitz, M.J., Ramani, A., Segur, H., A connection between nonlinear evolution equations and ordinary differential equations of F-type. I. J. Math. Phys. 21 (1980), no. 4, 715-721. MR565716 $(82 \mathrm{i}: 35148 \mathrm{a})$

[43] , A connection between nonlinear evolution equations and ordinary differential equations of F-type. II. J. Math. Phys. 21 (1980) no. 5, 1006-1015. MR.574872 (82i:35148b)

[44] Bruno, A.D., Power geometry as a new calculus, Analysis and Applications, H. G. W. Begehr, R. P. Gilbert and M.W.Wong (eds.), Kluwer, Dordrecht, 2003, pp. 51-71. MR2022739 (2004k:34013)

[45] Chang, Y.F., Greene, J.M., Tabor, M., Weiss, J., The analytic structure of dynamical systems and self-similar natural boundaries. Physica D 8 (1983), 183-207. MR.724588 (85a:58041)

[46] Dubrovin, B., Mazzocco, M., Monodromy of certain Painlevé-VI transcendents and reflection groups. Invent. Math. 141 (2000), 55-147. MR.1767271 (2001j:34114)

[47] Fuchs, L., Über Differentialgleichungen deren integrale feste Verzweigungspunkte besitzen. Sitz. Acad. Wiss. Berlin, 1884, 669-720.

[48] Fuchs, R., Sur quelques eq́uations différentielles linéaires du second ordres. C. R. Acad. Sci. Paris 141 (1905), 555-558.

[49] Gambier, B., Sur les équations différentielles du second ordre et du premier degré dont l'intégrale générale est a points critiques fixes. Acta Math. 33 (1910), 1-55. MR.1555055 
[50] Garnier, R., Sur des équations différentielles du troisième ordre dont l'intégrale générale est uniforme et sur une classe d'équations nouvelles d'ordre supérieur dont l'intégrale générale a ses points critiques fixes, Ann. Sci. De l'Ecole Normale Superieure 29 (1912), 1-126. MR1509146

[51] _ Etude de l'intégrale générale de l'équations VI de M. Painlevé. Ann. Ec. Norm. (3) 34 (1917), 243-353.

[52] Goryuchkina, I. V., About power-logarithmic expansions of solutions to the sixth Painlevé equation. Intern. Conf. on Differential Equations and Dynamical Systems, Suzdal, July 5-10, 2004, Sobor, Vladimir, 2004, pp. 259-260.

[53] _ _ Asymptotic expansions of solutions to the sixth Painlevé equation. ACA 2006. 12th. International Conference on Applications of Computer Algebra. Abstracts of Presentations. Sofia, 2006. p. 50.

[54] Gromak, I. V., Laine, I., Shimomura, S., Painlevé Differential Equations in the Complex Plane. Walter de Gruyter, Berlin-New York, 2002. MR:1960811 (2003m:34210)

[55] Guzzetti, D., On the critical behavior, the connection problem and elliptic representation of a Painlevé VI equation. Mathematical Physics, Analysis and Geometry 4 (2001), 293-377. MR:1888007 (2002m:34134)

[56] , The elliptic representation of the general Painlevé VI equation. Comm. Pure Appl. Math. 55 (2002), 1280-1363. MR1912098|(2003f:34187)

[57] The elliptic representation of the sixth Painlevé equation. Séminaires et Congrès 14 (2006), 83-101. MR2353463 (2009b:34276)

[58] _ Matching procedure for the sixth Painlevé equation. J. Phys. A 39 (2006), 11973-12031. MR:2266210 (2007h:34163)

[59] _ The logarithmic asymptotics of the sixth Painlevé equation. J. of Phys. A 41 (2008). 205201 (46 pp.). MR2450511 (2009g:34221)

[60] Iwasaki, K., Kimura, H., Shimomura, S., Yoshida, M., From Gauss to Painlevé. A modern theory of special functions. Vieweg, 1991. MR.1118604 (92j:33001)

[61] Jimbo, M., Monodromy problem and the boundary condition for some Painlevé transcendents. Publ. RIMS Kyoto Univ. 18 (1982), 1137-1161. MR688949 (85c:58050)

[62] Kimura, H., The construction of a general solution of a Hamiltonian system with regular type singularity and its application to Painlevé equations. Ann. Mat. Pura Appl. 134 (1983), 363-392. MR736747 (85d:34005)

[63] Mazzocco, M., Picard and Chazy solutions to the Painlevé VI equation. Math. Ann. 321 (2001), 157-195. MR 1857373 (2002g:34203)

[64] Okamoto, K., Studies on the Painlevé equations, I. The sixth Painlevé equation $P_{V I}$. Ann. Mat. Pura Appl. 146 (1987), 337-381. MR916698 (88m:58062)

[65] Painlevé, $P$., Leçons sur la théorie analytique des équations différentielles, professées à Stokholm. Paris, 1897.

[66] _ Mémoire sur les équations différentielles dont l'intégrale générale est uniforme. Bull. Soc. Math. France 28 (1900), 201-261. MR 1504376

[67] _ Sur les équations différentielles du second ordre et d'ordre supérieur, dont l'intégrale générale est uniforme. Acta Math. 25 (1902), 1-86. MR.1554937

[68] Picard, E., Démonstration d'un théorème générale sur les fonctions uniformes liées par une relation algébrique. Acta Math. 11 (1887), 1-12. MR 1554745

[69] _ Mémoire sur la théorie des fonctions algébriques de deux variables. Journal de Liouville 5 (1889), 135-319.

[70] Poincaré, H., Sur les intégrales irrégulières des équations linéaires. C. R. Acad. Sci. Paris. 101 (1885), 939-941. Oeuvres. Vol. IV, 611-613.

[71] , Sur les intégrales irrégulières des équations linéaires. C. R. Acad. Sci. Paris 101 (1885), 990-991. Oeuvres. Vol. IV, 614-615.

[72] _ Sur les intégrales irrégulières des équations linéaires, Acta Math. 8 (1886), 295-344. Oeuvres. Vol. I, 290-332.

[73] Shimomura, S., Painlevé transcendents in the neighbourhood of fixed singular points. Funkcial. Ekvac. 25 (1982), 163-184. MR694910 (86a:34031)

[74] _ Series expansions of Painlevé transcendents in the neighbourhood of a fixed singular point. Funkcial. Ekvac. 25 (1982), 185-197. MR694911 (86f:34017a)

[75] _ Supplement to "Series expansions of Painlevé transcendents in the neighbourhood of a fixed singular point". Funkcial. Ekvac. 25 (1982), 363-371. MR0707567 (86f:34017b)

[76] _ A family of solutions of a nonlinear ordinary differential equation and its application to Painlevé equations (III), (V), (VI). J. Math. Soc. Japan 39 (1987), 649-662. MR0905630|(88i:34027) 
[77] Takano, K., Reduction for Painlevé equations at the fixed singular points of the first kind. Funkcial. Ekvac. 29 (1986), 99-119. MR865217 (88i:34082)

[78] Watanabe, H., Birational canonical transformations and classical solutions of the sixth Painlevé equations. Ann. Scuola Norm. Sup. Pisa Cl. Sci. 27 (1999), 379-425. MR.1678014 (2000e:34154)

Keldysh Institute of Applied Mathematics, Russian Academy of Sciences, 4 Miusskaya Ploshchad', Moscow 125047, Russia

E-mail address: abruno@keldysh.ru

Keldysh Institute of Applied Mathematics, Russian Academy of Sciences, 4 Miusskaya Ploshchad', Moscow 125047, Russia

E-mail address: chukhareva@yandex.ru

Translated by O. KHLEBORODOVA 